\title{
Characterization of O-Glycosylation and Phosphorylation on Nuclear Protein by Mass Spectrometry
}

\author{
Andrew William Dawdy
}

Greenville, Illinois

B.A., Chemistry, Taylor University, 2009

\author{
A Dissertation presented to the Graduate Faculty \\ of the University of Virginia in Candidacy for the Degree of \\ Doctor of Philosophy \\ Department of Chemistry \\ University of Virginia \\ December, 2013
}


Thank you to Dr. Donald F. Hunt and Dr. Jeffrey Shabanowitz for allowing me to pursue my PhD in the Hunt Lab. I have learned an incredible amount during my doctoral education and research in this lab. My time here has well-equipped me for beginning the next chapter of my scientific career, and I don't take this opportunity that I have had for granted. I am very grateful, thank you.

Thank you to Drs. David S. Cafiso, Cameron Mura, Ralph O. Allen, and Daniel R. Foltz for serving on my graduate defense committee. I appreciate your time and contributions to my dissertation.

Thank you also to my many collaborators. I have enjoyed working with Drs. Tai-ping Sun, Kathy Wilson, Gerald Hart, Noel Lowndes, James Bieker, Peng Wu, and the outstanding graduate students and post-docs from these fine labs.

Much of my education and joy in the Hunt Lab has come from being surrounded by my intelligent and entertaining lab mates. Thank you to Dr. Dina Bai for enabling all our critical computing tools and for being an ever-positive force in the lab. Thank you to my senior lab mates, Drs. Jeremy Balsbaugh, Sushmit Maitra, Michelle English, Joseph Strukl, Aaron Bailey, Jenny Cottine, Patrick James, Joshua Nicklay, Jessica Chapman, Andrew Norris, Andrea DeSantis, and Erin Jeffrey. Thank you also to my peers and junior labmates, namely Drs. Weihan Wang, Peng Zhao, and Jenn Abelin, and Lissa Anderson, Lichao Zhang, Stacy Malaker, Amanda Wriston, Marshall Chaffee, Ben Barnhill, Scott Ugrin, Paisley Trantham, and Garrett Tanner. I learned so much from you, and I have a collection of fond memories with you all. Thank you especially to the "rowdy" side of the lab for all the laughs. These many memories will always bring a smile to 
my face. Thank you also to the "quiet" side for providing a refuge away from the craziness. It has been a pleasure and honor to work alongside all of you. I wish you all well.

Thank you to all my educators in the Bond County Community Unit \#2 school district, Taylor University, and the University of Virginia. The road to a PhD starts early, and it was paved in part by many great teachers and professors. I would like to particularly acknowledge and thank my high school chemistry teacher, Mr. Brian Grove, for creating a fun and educational atmosphere that sparked my first serious interest in chemistry.

Above all those previously named, thank you to my wonderful family. Mom and Dad, you are excellent parents, and you always gave me the love and support necessary for achieving my goals. One of those goals has now been realized in my attainment of a $\mathrm{PhD}$, and I thank you for that. Thank you also to my siblings, Becky, Danny, and Sarah, for your support of "The Baby" over the years. I have so many fond memories growing up with you, and I look forward to making more memories with all of our families. Thank you to Pop and Gam Dawdy and Grandpa and Grandma Johnson for your love and prayers as I grew up, and for beginning this amazing family of which I am proud and privileged to be a part. Mom, Dad, Becky, Danny, Sarah, and my entire extended family, I am blessed to have you all as my family, and I love you very much.

During my first summer as a graduate student in the Hunt Lab, I was fortunate to join another wonderful family as well. Mitch, Pam, Garrett, and Ris, thank you for allowing me to marry Natalie and for adopting me into your family. You have been so welcoming and loving, and I look forward to beginning life as a Floridian with you. I love you all.

The person who I thank the most is my incredible wife, Natalie, the most amazing young woman on this earth. I believe our marriage is as close to perfect as humanly possible. I am 
Preface

truly the most blessed man to have you as my wife. Thank you for being my best friend and

companion. I have loved these first few years of marriage with you, and I am so looking forward to beginning this next chapter of our lives together. I love you as much as a man can love his wife. Thank you for all of your love and support.

Ultimately, I thank God for giving me a mind and body capable of studying His awesome world. May the skills I have learned always be used to glorify You, and may my ever-increasing knowledge of Your world bring me ever-increasingly closer to You. 


\section{Table of Contents}

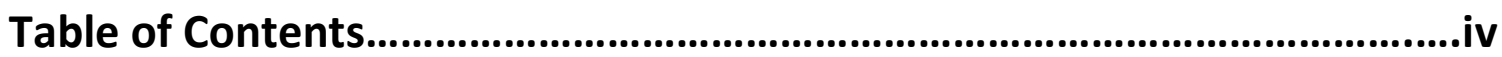

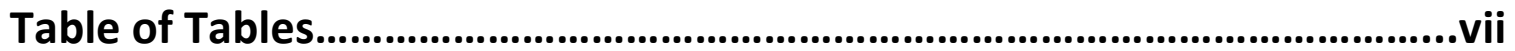

Table of Figures...........................................................................................................viii

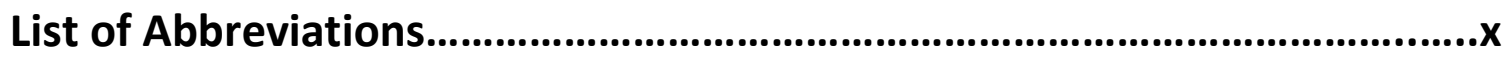

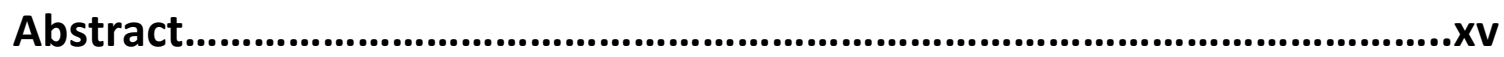

\section{Chapter 1: Introduction to the Dissertation}

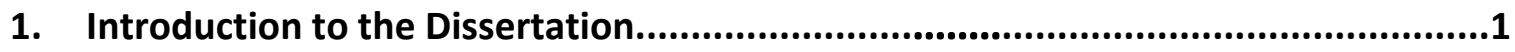

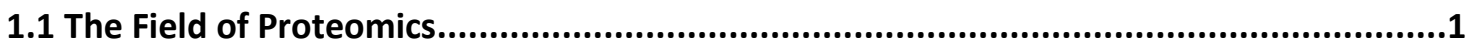

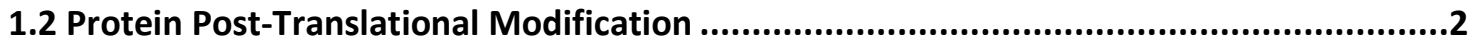

1.3 Characterizing PTMs by Mass Spectrometry .....................................................4

1.4 References.............................................................................................................14

\section{Chapter 2: Characterization of O-GIcNAcylation, phosphorylation, and a} novel O-linked nuclear PTM, O-hexose, on DELLA protein RGA

2.1 Introduction ........................................................................................... 16

2.1.1 Post-translational modification of proteins is ubiquitous and essential ....................16

2.1.2 Post-translational modification O-linked $\beta$ - $\mathrm{N}$-acetylglucosamine............................17

O-GIcNAc transferase catalyzes the addition of O-GIcNAc to Ser and Thr ............................. 19

O-GIcNAcase catalyzes the removal of O-GIcNAc from Ser and Thr .....................................20

O-GIcNAc donor molecule, UDP-GIcNAc, is produced by the Hexosamine Biosynthetic

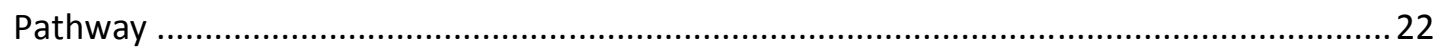

O-GIcNAcylation occurs on wide variety of proteins involved in virtually all intracellular

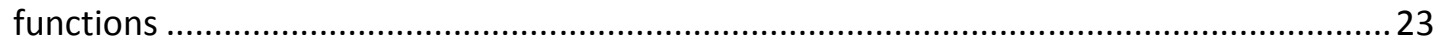

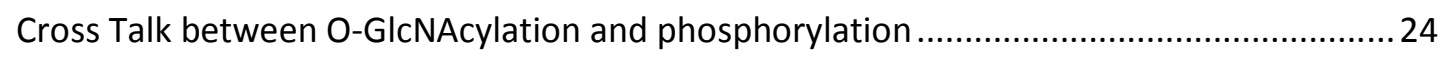

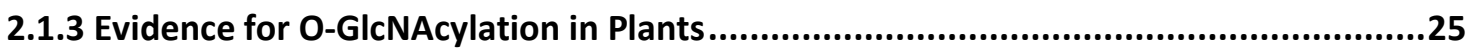

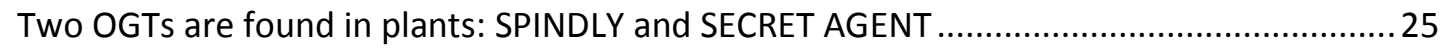

Evidence for O-GIcNAcylation of DELLA protein Repressor of ga1-3 (RGA) ..........................26

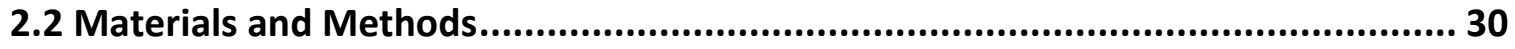

2.2.1 Proteolytic Digestions and Sample Preparation for Mass Spectrometric Analysis ........30

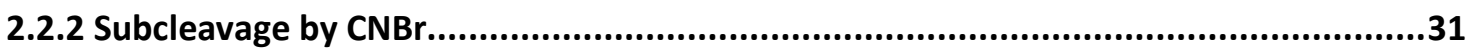


2.2.3 Derivatization of Carboxylic Acids with Histamine.......................................................31

2.2.4 HPLC Capillary Analytical and Pre-column Assembly ........................................32

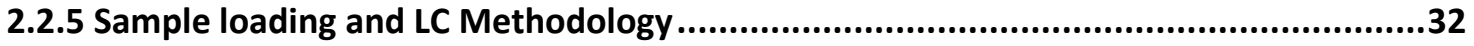

2.2.6 Phosphorylated Peptide Enrichment by Immobilized Metal Affinity Chromatography33

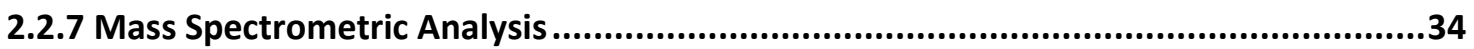

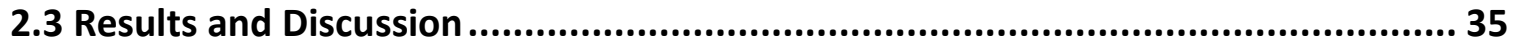

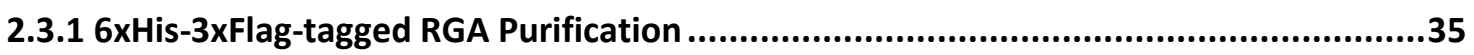

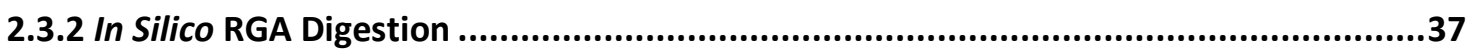

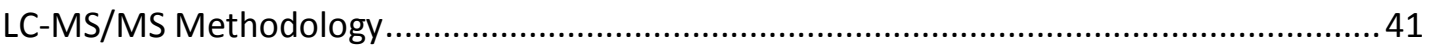

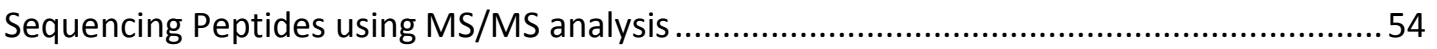

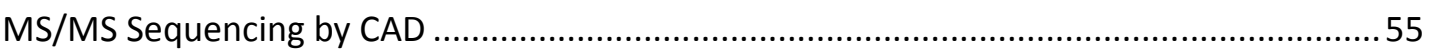

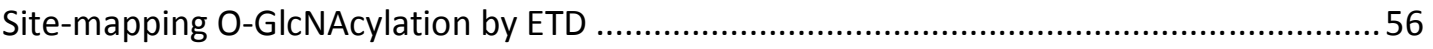

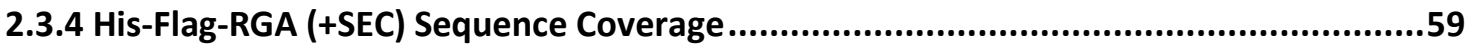

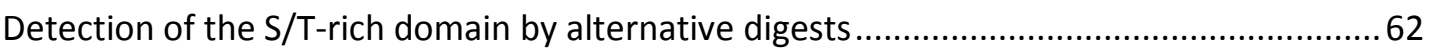

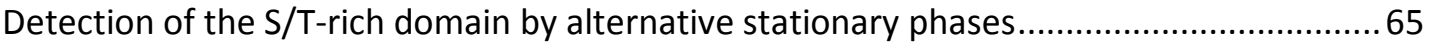

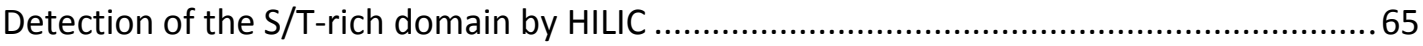

Detection of the S/T-rich domain by Derivatization of Carboxylic Acids with Histamine .....66

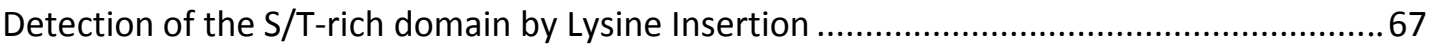

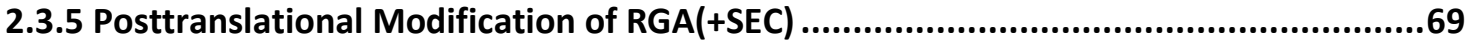

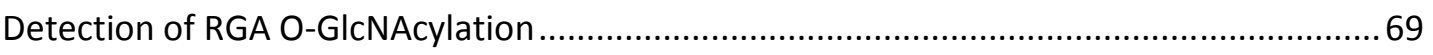

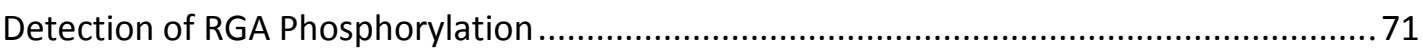

Detection of an unexpected and unprecedented glyco-PTM .............................................. 71

Stoichiometry and Site-locations of RGA O-GIcNAcylation, Phosphorylation, and O-

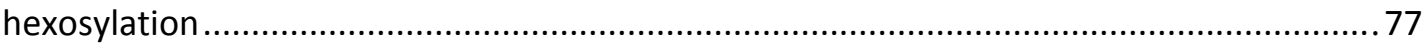

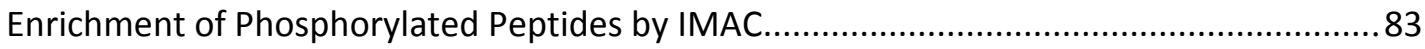

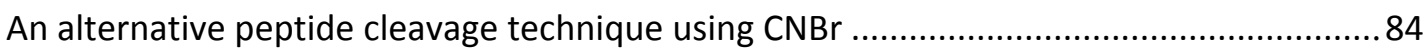

Enhancement of ETD by Derivatization of Carboxylic Acids with Histamine....................... 92

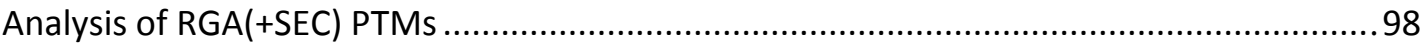

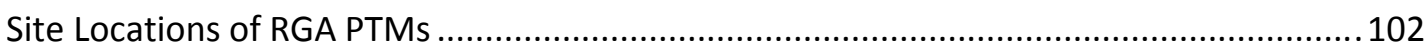

2.3.6 The effect of SEC on O-hexosylation .................................................................... 108

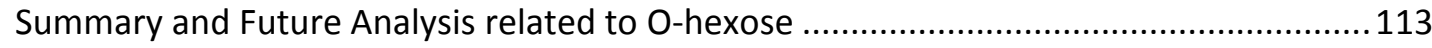

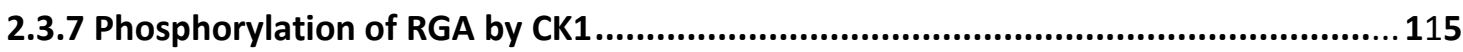

2.4 Conclusions .........................................................................................119

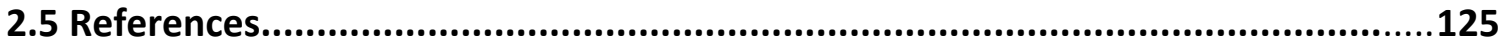

\section{Chapter 3: Site-specific phosphorylation of the DNA damage response mediator rad 9 by cyclin-dependent kinases regulates activation of checkpoint kinase 1}

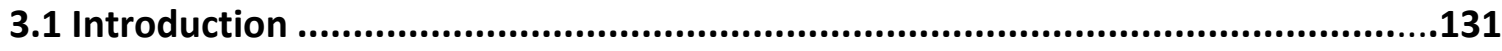

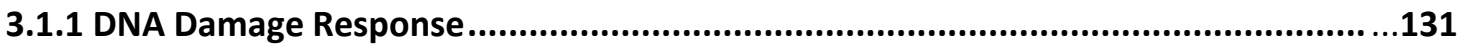




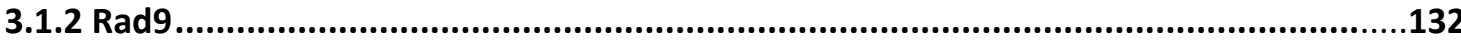

3.1.3 Checkpoint Kinase 1 (Chk1) Activation by Rad9.................................................. 133

3.2 Materials and Methods.............................................................................134

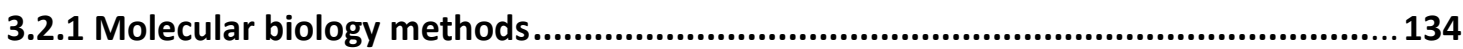

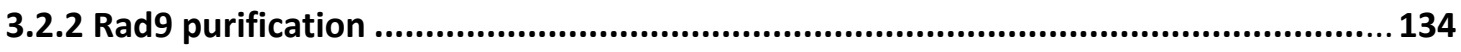

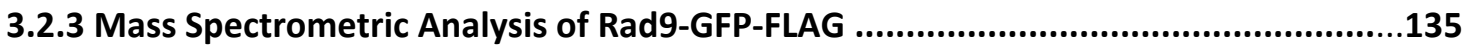

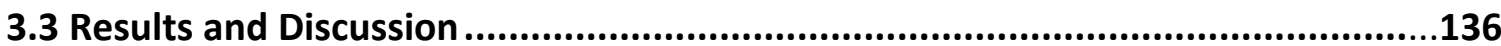

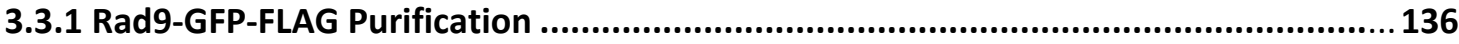

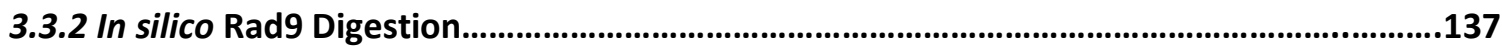

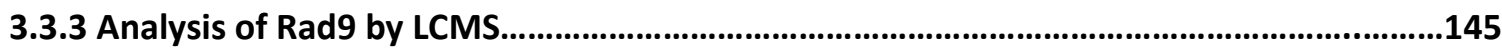

3.3.4 Biological role of CAD phosphorylation in Chk1 activation..............................................154

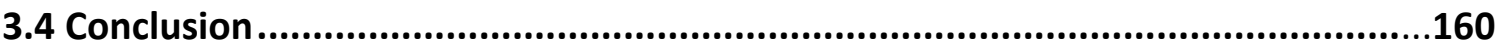

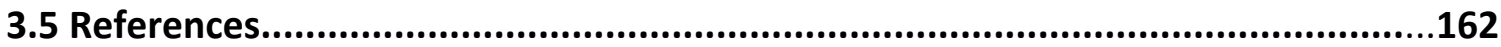




\section{Table of Tables}

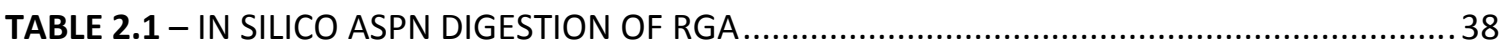

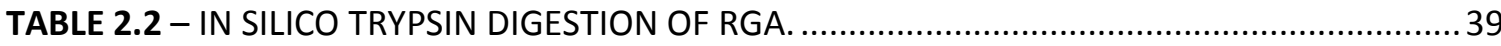

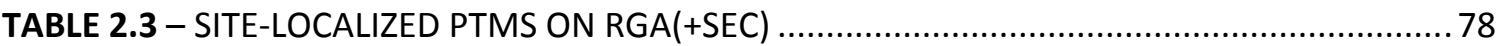

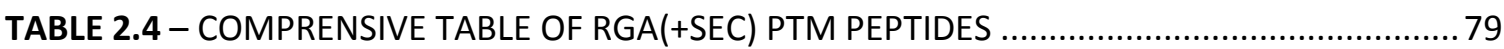

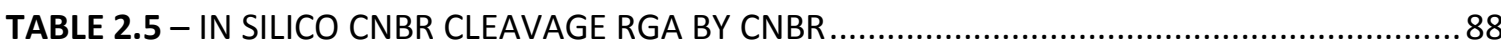

TABLE 2.6 - IN SILICO SUBCLEAVAGE OF APN-N DIGESTED RGA BY CNBR ................................90

TABLE 2.7 - CONDENSED SUMMARY OF THE 13 MODIFIED RGA(+SEC) PEPTIDES ......................99

TABLE 2.8 - EXPANDED SUMMARY OF THE 13 MODIFIED RGA(+SEC) PEPTIDES ...................... 100

TABLE 2.9 - COMPREHENSIVE TABLE OF RGA(-SEC) PTM PEPTIDES .........................................109

TABLE 2.10 - SUMMARY OF THE 4 MODIFIED RGA(-SEC) PEPTIDES .....................................110

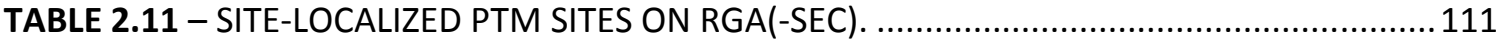

TABLE 2.12 - COMPREHENSIVE TABLE OF RGA(+CK1) PTM PEPTIDES .....................................116

TABLE 2.13 - SUMMARY OF THE 6 MODIFIED RGA(+CK1) PEPTIDES........................................117

TABLE 2.14 - SITE-LOCALIZED PTM SITES ON RGA(+CK1)................................................. 118

TABLE 2.15 - COMPREHENSIVE TABLE OF RGA(+CK1) PTM PEPTIDES ….................................120

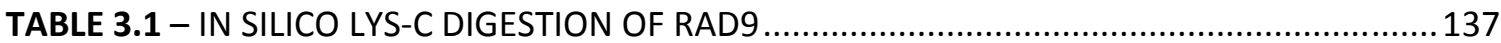

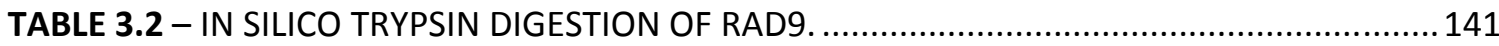

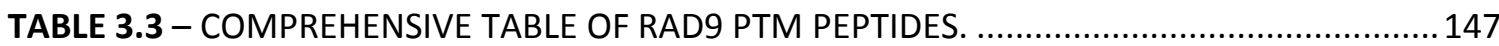

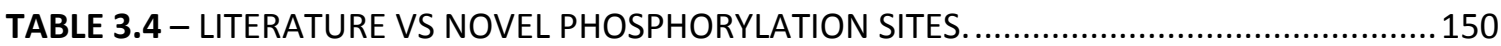

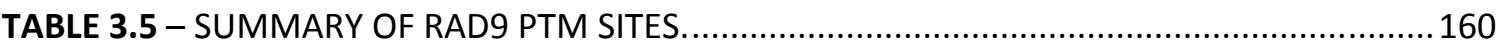




\section{Table of Figures}

FIGURE 1.1 - FIRST STABILITY REGION DEFINED BY MATHIEU EQUATION

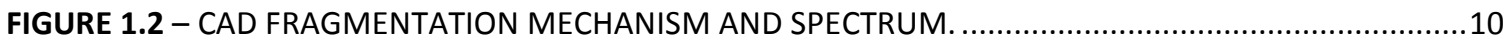

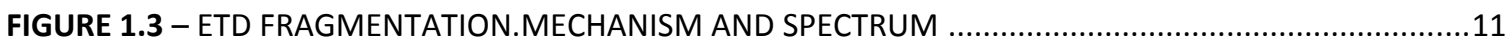

FIGURE 2.1 - O-GLCNAC TRANSFERASE AND O-GLCNACASE RESPECTIVELY INSTALL AND REMOVE O-

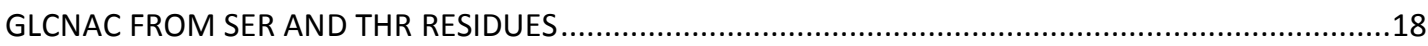

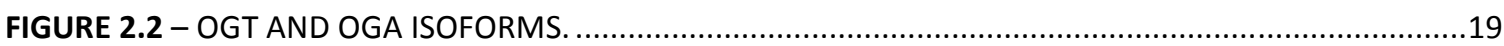

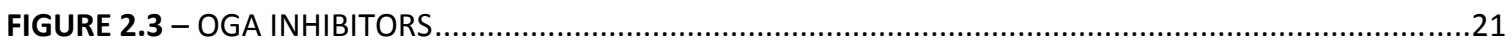

FIGURE 2.4 - THE HEXOSAMINE BIOSYNTHETIC PATHWAY PRODUCES O-GLCNAC DONOR, UDP-GLCNAC. 22

FIGURE 2.5 - O-GLCNACYLATION MODIFIEDS PROTEIN FROM ALL FUNCTIONAL CLASSES ........................23

FIGURE 2.6 - O-GLCNACYLATED PROTEINS ARE INVOLVED IN A WIDE VARIETY OF CELLULAR PROCESSES. 24

FIGURE 2.7 - ARABIDOPSIS SPY (TOP) AND HUMAN OGT (BOTTOM) ARE HIGHLY HOMOLOGOUS.............26

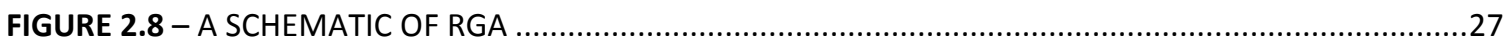

FIGURE 2.9 - DELLA PROTEIN RGA AND OGT PROTEIN SPY EACH BOTH HAVE ROLES IN NEGATIVELY

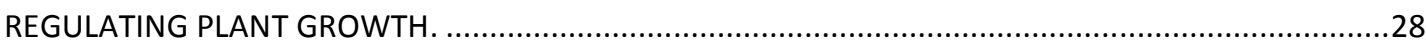

FIGURE 2.10 - FIVE DELLA PROTEINS HAVE BEEN DISCOVERED IN ARABIDOPSIS. RGA IS A CENTRAL

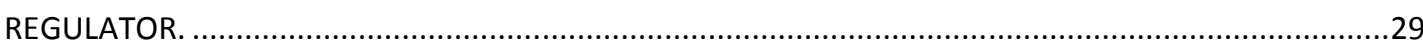

FIGURE 2.11 - GAS STIMULATE PLANT DEVELOPMENT BY INDUCING DEGRADATION OF THE REPRESSOR

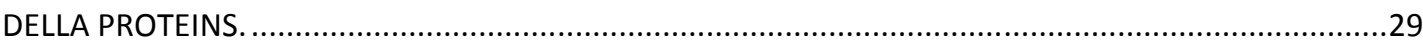

FIGURE 2.12 - RGA VISUALIZED BY COOMASSIE STAIN ON SDS-PAGE GEL. .........................................36

FIGURE 2.13 - WESTERN BLOT (ANTI-FLAG-HRP) SHOWS CHANGES IN THE SDS-PAGE GEL SHIFT WHEN POTENTIAL SER/THR-RICH REGIONS OF RGA ARE MUTATED TO ALA, ELIMINATING THEIR ABILITY TO BE O-GLCNACYLATED.

FIGURE 2.14 - THEORETICAL ISOTOPIC DISTRIBUTION OF VASOACTIVE INTESTINAL PEPTIDE FRAGMENT, HSDAVFTDNYTR.

FIGURE 2.15 - EXPERIMENTAL ISOTOPIC DISTRIBUTION OF VASOACTIVE INTESTINAL PEPTIDE FRAGMENT, HSDAVFTDNYTR, USING LTQ-FT AT 50,000 FWHM RESOLUTION.

FIGURE 2.16 - XICS OF INTERNAL PEPTIDE STANDARDS VASOACTIVE INTESTINAL PEPTIDE (VASO) AND ANGIOTENSIN (ANGIO) USED TO CALCULATE RELATIVE QUANTITATION OF RGA PEPTIDES...............50

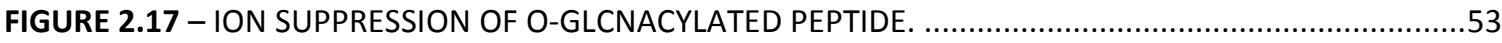

FIGURE 2.18 - SCHEMATIC OF 4 SER/THR-RICH DOMAINS IN RGA ..................................................56

FIGURE 2.19 - CAD AND ETD SPECTRA OF MONO-O-GLCNACYLATED PEPTIDE DHHQFQGRLSNHGTSSSSSSIS.

FIGURE 2.20 -BASE PEAK CHROMATOGRAMS OF REPRESENTATIVE TRYPSIN AND ASPN RGA DIGESTS.....60

FIGURE 2.21 - RGA SEQUENCE COVERAGE FROM TRYPSIN AND ASPN..

FIGURE 2.22- SEPARATION OF PEPTIDE SCSSPDSMVTSTSTGTQIGGVIGTTVTTTTTTTTAAGESTR BY FOUR DIFFERENT CHROMATOGRAPHIC STATIONARY PHASES, AND C18 AFTER HISTAMINE DERIVATIZATION OR TRYPSIN+ASPN DIGESTION.

FIGURE 2.23 - REACTION MECHANISM FOR DERIVATIZATION OF CARBOXYLIC ACIDS WITH HISTAMINE. .67 FIGURE 2.24 - WESTERN BLOT DEMONSTRATING CHARACTERISTIC RGA(+SEC) SHIFT WAS PRESERVED FOR

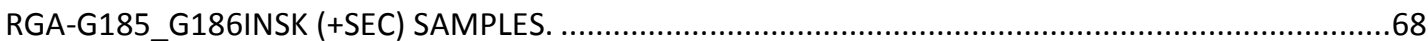

FIGURE 2.25 - XICS OF THE PREVIOUSLY UNDETECTABLE RGA(+SEC) POLY-SER/THR REGION...................69 FIGURE 2.26 - XICS OF 1-6 O-GLCNACS DETECTED ON GVIGTTVTTTTTTTTAAGESTR................................70 FIGURE 2.27 - DETECTION OF A PREVIOUSLY UNDESCRIBED NUCLEAR PROTEIN PTM, O-HEXOSYLATION. 
Preface

Table of Figures

FIGURE 2.28 - XICS OF 1-5 UNEXPECTED $\triangle 162$ MASSES ON GVIGTTVTTTTTTTTAAGESTR DETERMINED TO BE O-HEXOSE PTMS.

FIGURE 2.29 - HEXOSE PTM SIGNATURE FRAGMENT IONS PRODUCED BY CAD, CHARACTERISTIC OF OLINKAGE.

FIGURE 2.30 - IMAC STATIONARY PHASE

FIGURE 2.31 - ETD \% FRAGMENTATION IS RELATIVELY INDEPENDENT OF CHARGE STATE AND PEPTIDE MASS, BUT IS HIGHLY DEPENDENT ON CHARGE DENSITY...

FIGURE 2.32 - REACTION SCHEME FOR CLEAVAGE OF MET-X BONDS BY CNBR

FIGURE 2.33 - ETD CONSUMES ONE CHARGE, REQUIRING A MINIMUM OF 3 CHARGES FOR C AND Z· IONS TO BOTH BE DETECTED.

FIGURE 2.34 - DERIVATIZATION OF ASP AND THE C-TERMINUS OF UNMODIFIED VIPGNAIYQFPAIDSSSSSNNQNK WITH HISTAMINE

FIGURE 2.35 - REACTION SCHEME OF ASP RESIDUES FORMING STABLE SUCCINIMIDE SIDE-PRODUCTS....95

FIGURE 2.36 -LOCALIZATION OF O-GLNAC AND O-HEXOSE ON ASN-P+CNBR PEPTIDE LSELNPPPLPASSNGL DERIVATIZED WITH HISTAMINE.

FIGURE 2.37 - HEAT MAP REPORTING ABUNDANCES OF THE 26 POST-TRANSLATIONALLY MODIFIED FORMS OF GVIGTTVTTTTTTTTTAAGESTR.

FIGURE 2.38 - MIXED ETD SPECTRUM OF MONO-O-GLCNACYLATED GVIGTTVTTTTTTTTAAGESTR IN RGA($\mathrm{SEC})$...

FIGURE 2.39 - SUM TOTAL OF O-GLCNAC, PHOSPHATE, AND O-HEXOSE IN RGA(+SEC,-SEC,\&+CK1) FOR THE SIX MOST ABUNDANTLY MODIFIED RGA PEPTIDES.

FIGURE 2.40 - RGA SEQUENCES SUMMARIZING RESIDUES MODIFIED WITH COMBINATORIAL PTMS ......122

FIGURE 3.1 - TANDEM PURIFIED RAD9-GFP-FLAG VISUALIZED BY SILVER STAINED SDS-PAGE GEL ..........137

FIGURE 3.2 - SEQUENCE COVERAGE OF TRYPSIN AND LYS-C-DIGESTED RAD9 FROM ASYNCHRONOUS, G1-, AND G2-ARRESTED CELLS. .145

FIGURE 3.3 - SCHEMATIC OF RAD9 PHOSPHORYLATION SITES DETECTED .154

FIGURE 3.4 - (A) SCHEMATIC OF RAD9 CAD HIGHLIGHTING CDC28 CONSENSUS SITES 1-9 MUTATED TO ALA IN RAD9CDK1-9A PROTEIN. (B) CDC28/CLB2 PRODUCES THE HIGHEST LEVEL OF IN VITRO PHOSPHORYLATION OF RAD9 CAD ${ }^{\mathrm{WT}}$. (C) DNA-DAMAGE-DEPENDENT PHOSPHORYLATION OF CHK1 IS REDUCED OR ELIMINATED IN RAD9CDK1-9A, RAD9CADA, RAD9 $\triangle$ CELLS. (D\&E) - CHK1 ACTIVATION IS PRINCIPALLY DEPENDENT ON CDK CONSENSUS SITES 1-9 IN G2/M CELLS.

FIGURE 3.5 - CHK1 ACTIVITY WAS DEPENDENT ON CDC28 UPON DNA DAMAGE SEPARATELY INDUCED BY BLEOCIN OR 4-NQO.

FIGURE 3.6 - (A) RAD9/CHK1 INTERACTION WAS DEPENDENT ON CDK1-9 BASED ON YEAST TWO-HYBRID ASSAY IN VIVO. (B) RAD9/CHK1 INTERACTION WAS DEPENDENT ON CDC28 IN G2/M AND WAS CDC28-INDEPENDENT IN G1.

FIGURE 3.7 - (A) YEAST-TWO HYBRID ASSAY DEMONSTRATED THAT T125 AND T143 MOST CONTRIBUTED TO CAD/CHK1 INTERACTION OF CDK1-9 SITES. (B) - PEPTIDES CONTAINING PT143 MOST EFFECTIVELY PULLED DOWN CHK1 WHILE PT125 SHOWED AN INHIBITORY EFFECT. 159

FIGURE 3.8 - MODEL OF CHK1 ACTIVATION IN RESPONSE TO DNA DAMAGE. 


\section{Abbreviations}

${ }^{\circ} \mathrm{C}$

A

$\mathrm{AcOH}$

AGC

Ala, A

Angio

Ambic

Arg, R

Asn, N

Asp, D

ATM

ATP

ATR

B

c

C

C18

CAD

CDK

Chk1

Chk2

$\mathrm{Cl}$

$\mathrm{CNBr}$

Cys, C

$\mathrm{Da}$
Degrees Celsius

Radical species

Solvent $A, 100 \mathrm{mM}$ acetic acid in water

Acetic Acid

Automated gain control

Alanine

Angiotensin I

Ammonium bicarbonate

Arginine

Asparagine

Aspartic acid

Ataxia telangiectasia, mutated

adenosine triphosphate

ATM and Rad3-related

Solvent B, $100 \mathrm{mM}$ acetic acid in 70\% acetonitrile

Centi $\left(10^{-2}\right)$

Carboxyl terminus of a polypeptide

Octadecylsilane

Collison-activated dissociation or Chk1 activating domain

Cyclin-dependent kinase

Checkpoint kinase 1

Checkpoint kinase 2

Chemical ionization

Cyanogen bromide

Cysteine

Dalton 


\begin{tabular}{|c|c|}
\hline Preface & List of Abbreviations \\
\hline$\overline{\mathrm{DC}}$ & Direct current \\
\hline DDR & DNA Damage Response \\
\hline DNA & deoxyribonucleic acid \\
\hline DTT & Dithiothreitol \\
\hline ECD & Electron-capture dissociation \\
\hline EDC & 1-ethyl-3-(3-dimethylaminopropyl)carbodiimide \\
\hline EDTA & Ethylenediaminetetraacetic acid \\
\hline El & Electron ionization \\
\hline EM & Electron multiplier \\
\hline ER & endoplasmic reticulum \\
\hline ESI & Electrospray ionization \\
\hline ETD & Electron-transfer dissociation \\
\hline$f$ & Femto $\left(10^{-15}\right)$ \\
\hline FAB & Fast atom bombardment \\
\hline $\mathrm{FeCl} 3$ & Iron (III) chloride \\
\hline FT-ICR & Fourier transform ion cyclotron resonance \\
\hline FWHM & Full width half max \\
\hline g & Gram \\
\hline G1 & Gap 1 phase \\
\hline G2 & Gap 2 phase \\
\hline Gal & Galactose \\
\hline$G \ln , Q$ & Glutamine \\
\hline Glu, E & Glutamic acid \\
\hline Gly, G & Glycine \\
\hline gS, gT & O-GIcNAcylated residue \\
\hline hS, hT & O-hexosylated residue \\
\hline $\mathrm{h}, \mathrm{hr}$ & Hour \\
\hline HBP & Hexosamine biosynthetic pathway \\
\hline
\end{tabular}




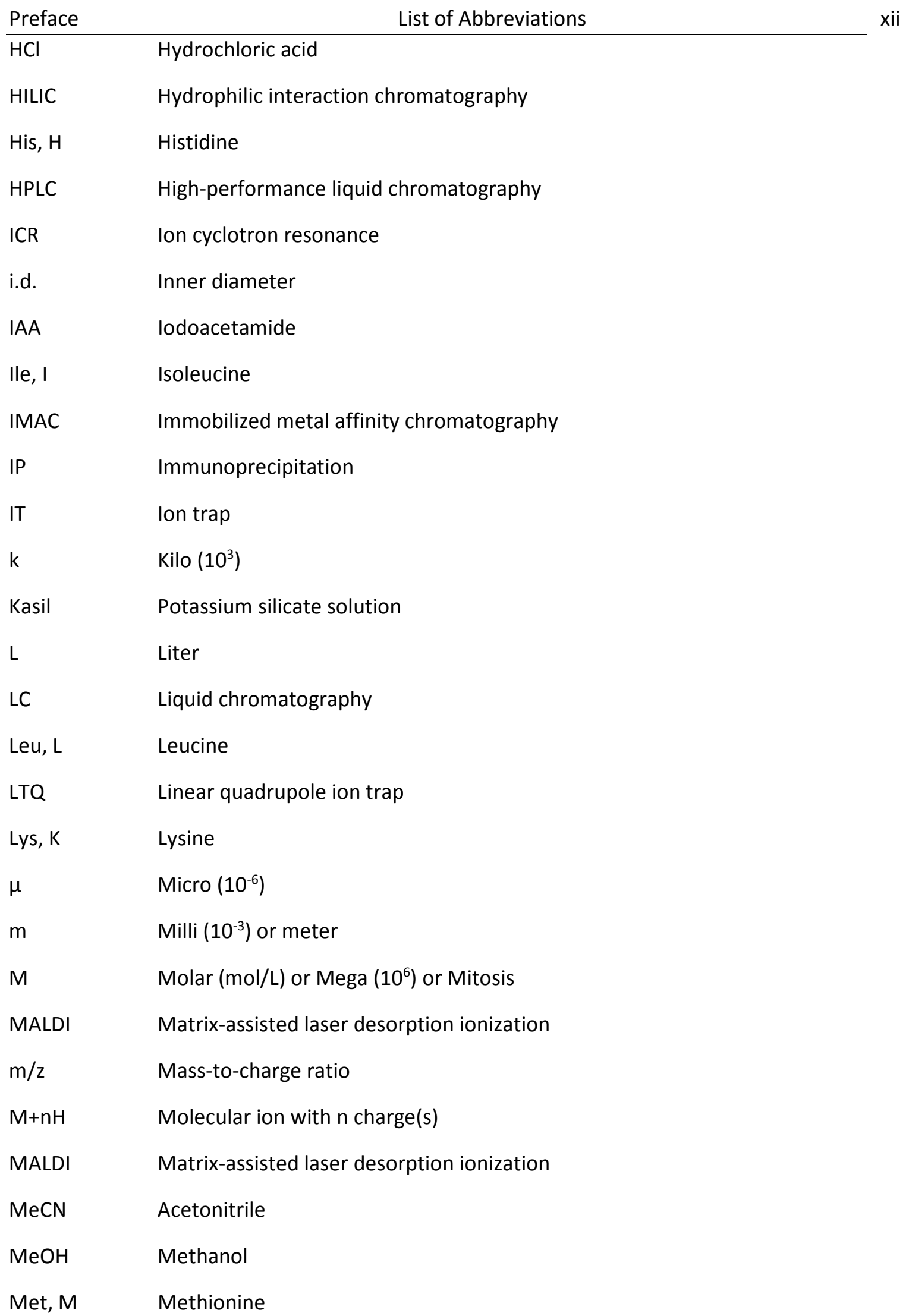




\begin{tabular}{|c|c|}
\hline Preface & List of Abbreviations \\
\hline $\min$ & Minute \\
\hline $\mathrm{mol}$ & Mole $\left(6.022 \times 10^{23}\right)$ \\
\hline MS & Mass spectrometry \\
\hline MS/MS & Tandem mass spectrometry \\
\hline $\mathrm{MS}^{1}$ & Full mass spectrum \\
\hline $\mathrm{MS}^{2}$ & Tandem mass spectrum \\
\hline MW & Molecular weight \\
\hline$n$ & Nano $\left(10^{-9}\right)$ \\
\hline N & Amino terminus of a polypeptide \\
\hline NCBI & National Center for Biotechnology Information \\
\hline NHS & N-hydroxysuccinimide \\
\hline $\mathrm{Ni}^{2+}-\mathrm{NTA}$ & Nickel (II) - nitriloacetic acid \\
\hline NL & Normalized level ion counts \\
\hline o.d. & Outer diameter \\
\hline OGA & O-GlcNAcase \\
\hline O-GlcNAc & O-linked $\beta$-N-acetylglucosamine \\
\hline OGT & O-GIcNAc transferase \\
\hline O-hexose & O-linked hexose \\
\hline OMSSA & Open Mass Spectrometry Search Algorithm \\
\hline $\mathrm{p}$ & Pico $\left(10^{-12}\right)$ \\
\hline PAGE & Polyacrylamide gel electrophoresis \\
\hline Phe, F & Phenylalanine \\
\hline ppm & Part-per-million \\
\hline Pro, $\mathrm{P}$ & Proline \\
\hline $\mathrm{pS}, \mathrm{pT}, \mathrm{pY}$ & Phosphorylated residue \\
\hline PTFE & Polytetrafluoroethylene \\
\hline PTM & Post-translational modification \\
\hline QIT & Quadrupole ion trap \\
\hline
\end{tabular}




\begin{tabular}{|c|c|}
\hline Preface & List of Abbreviations \\
\hline Rad9 & Prototypical DNA Damage Response Mediator protein \\
\hline RGA & 'Repressor of ga1-3' protein \\
\hline RF & Radio frequency \\
\hline RNA & Ribonucleic acid \\
\hline RP & Reverse phase \\
\hline S & Second(s) \\
\hline SDS & Sodium dodecyl sulfate \\
\hline SEC & SECRET AGENT \\
\hline Ser, $\mathrm{S}$ & Serine or Synthesis phase \\
\hline SPY & SPINDLY \\
\hline SUMO & Small ubiquitin-like modifier \\
\hline TFA & Triflouroacetic acid \\
\hline Thr, $\mathrm{T}$ & Threonine \\
\hline Trp, W & Tryptophan \\
\hline Tyr, Y & Tyrosine \\
\hline UDP & Uridine diphosphate \\
\hline Val, V & Valine \\
\hline Vaso & Vasoactive intestinal peptide \\
\hline$x$ & Any amino acid \\
\hline XIC & Extracted ion chromatogram \\
\hline WB & Western blot \\
\hline WT & Wild-type \\
\hline
\end{tabular}




\section{Abstract}

Proteins are biomolecules that represent the ultimate expression of DNA, as they perform the majority of structural and functional roles in organisms. Proteins accomplish this through their interactions with other proteins and biomolecules, dictated by their molecular confirmation and location. A major regulator of protein activity is covalent post-translational modification (PTM). Characterizing protein PTMs is critical for developing a complete understanding of the proteome, the entire expressed protein complement of DNA. Over 400 PTMs have been identified, and liquid chromatography mass spectrometry (LCMS) has emerged as the most powerful technique to directly characterize protein PTMs. This dissertation reports the implementation of electron transfer dissociation (ETD) and collision-activated dissociation (CAD) in tandem with high-resolution MS to characterize O-linked glycosylation and phosphorylation PTMs on two nuclear proteins. Phosphorylation is a ubiquitous nucleocytoplasmic PTM that is found on Ser, Thr, and Tyr residues of thousands of metazoan proteins. It is involved in the regulation of a wide variety of cellular processes and is perhaps the mostly highly-studied PTM to date. Just 30 years ago, it was largely believed that nucleocytoplasmic protein glycosylation was non-existent. We now know that the nucleocytoplasmic monosaccharide, O-linked $\beta-\mathrm{N}$-acetylglucosamine, or O-GlcNAc, modifies Ser and Thr residues of metazoan proteins from nearly every functional class, and it has a complex interplay with phosphorylation. O-GlcNAcylation has historically been challenging to study, but improvements in mass spectrometric techniques, such as the development of ETD that preserves labile O-linked PTMs like O-GIcNAc upon fragmentation, have significantly increased the ability to characterize this PTM. It is becoming increasingly evident that O-GlcNAcylation 
Preface

Abstract $x v i$

also regulates numerous cellular processes including transcription, translation, protein trafficking, and protein degradation.

Firstly, we report the comprehensive characterization of O-GlcNAcylation on Arabidopsis protein 'repressor of ga1-3', or RGA, a negative regulator of plant growth and development. This marks the first direct and comprehensive characterization of O-GIcNAcylation on a plant protein. In addition, numerous phosphorylation sites were discovered. Most notably and surprisingly, a previously unreported O-linked hexose monosaccharide PTM was discovered on RGA, representing only the second nucleocytoplasmic glyco-PTM reported to date.

Secondly, we report novel phosphorylation of S. cerevisiae DNA damage response mediator protein Rad9. Phosphorylation was detected on all 20 putative Cyclin-dependent kinase (CDK) motifs, including 7 previously unreported. All 9 CDK sites located in the Checkpoint kinase 1 (Chk1) activating domain (CAD) were phosphorylated and sites T125 and T143 were found to be especially important in facilitating Rad9-Chk1 interaction and Chk1 activation. 


\section{Chapter 1: Introduction to the Dissertation}

\subsection{The Field of Proteomics}

Proteomics is an analogous term to genomics, and was created to describe the study of the third and final core component in the central dogma of molecular biology, protein ${ }^{1,2}$. The first two molecular components, DNA and RNA, are comprised of nucleic acids. An organism's DNA contains all its hereditary information and collectively forms its genome. This genetic map may be transiently expressed by transcription of a DNA sequence to form the second molecular component of the central dogma, RNA. RNA, comprised of types ribosomal (rRNA), transfer (tRNA), messenger (mRNA), and regulatory non-coding (ncRNA), functions as the expressed form of genetic information by providing molecular machinery and the DNA-derived template necessary for protein synthesis.

The primary structure of a protein consists of a linear molecular chain, or polypeptide, built from combinations of 20 fundamental amino acids. Polypeptides range in size from $<10$ $\mathrm{kDa}$ to $>1 \mathrm{MDa}$ and typically fold into tertiary structures dictated directly by their primary sequence of amino acids. Two of the most common protein secondary structural motifs are $\alpha$ helices and $\beta$-sheets. The tertiary structure of the protein is formed by the collective interactions that facilitate folding into its final three-dimensional form. These interactions include hydrogen and disulfide bonding, and ionic and van der Waals interactions amongst different regions of the protein. A protein generally adopts a tertiary conformation that minimizes free energy and may be comprised of independently folding secondary structures, folding between secondary structures and/or undefined domains, and unfolded regions. Lastly, 
quaternary protein complexes may be created by non-covalent bonding of multiple polypeptide chains together that function as a single unit (e.g. hemoglobin) ${ }^{3}$.

In this way, amino acid sequences dictate higher order structure, and this ultimately dictates the interactions a protein will have with other molecules. Proteins perform most of the structural and functional roles in the cell and are the ultimate molecular product of expressed genes. The proteome may be defined as "the entire complement of proteins expressed in a specific state of an organism or a cell population" ${ }^{4,5}$. The ultimate goal of proteomics is the detailed characterization of the proteome.

There are $20,000-25,000$ protein-coding genes in the human genome ${ }^{6}$, yet the proteome is estimated to be $2-3$ orders of magnitude larger than this due to the numerous modifications that proteins may undergo. The two main routes to accomplish this are alternative gene splicing at the mRNA level, and enzymatic covalent post-translational modification (PTM) of proteins. The latter of these is the subject of this dissertation ${ }^{7}$.

\subsection{Protein Post-Translational Modification}

Post-translational modifications add to the complexity of the proteome and are often difficult to predict and detect, because they directly change the primary chemical structures of proteins beyond what is directly dictated by the DNA sequence. This influences protein structure, thereby influencing molecular interactions and protein function. PTMs occur in prokaryotes, but are more diverse in type and higher in occurrence in eukaryotes. Approximately $5 \%$ of higher eukaryotes genomes can be dedicated to enzymes that perform

protein post-translational modification ${ }^{7}$. Over 400 types of PTMs have been discovered ${ }^{8}$ with five most common types being phosphorylation, glycosylation, acylation, alkylation, and 
oxidation $^{7}$. A complete understanding of the proteome therefore requires the characterization of all the PTMs that alter it. The combinatorial power of multiple PTMs quickly and vastly increases the diversity of proteins' molecular states in a manner that allows intricate biological tasks to be performed on a developmental and physiological timescale ${ }^{3}$. This dissertation focuses on the study of two PTMs critical for the regulation of nuclear and cytoplasmic proteins: phosphorylation and O-linked $\beta$-N-acetylglucosamine (O-GlcNAc), a type of O-linked glycosylation ${ }^{9,10}$.

PTMs have historically been studied using a variety of biochemical techniques. Radioactive substrates have been used to perform in vitro PTM reactions assays, although these can be hazardous and relatively insensitive. Western blot analysis is also used to detect PTMs by raising antibodies against them. This can be done with monoclonal antibodies raised against a specific PTM site, or by generating pan-specific antibodies to recognize a type of PTM without regard to sequence. The first allows one to probe a specific site, but prior knowledge or an indication of the site must exist and generating specific antibodies is time intensive and expensive. The second approach allows one to assay a larger population of proteins for a particular PTM, but generating pan-specific antibodies for small molecule modifications, like many of the most common PTMs, can be difficult to do with high affinity and independently of the adjacent sequences. Both antibody methods suffer from the potential for ambiguity in results due to the inevitable lack of specificity for $100 \%$ of modified sites and potential infidelity of the antibody. They are also not reliable when attempting to determine a library of combinatorial PTMs on one or more proteins. In another more recent approach, peptide and protein arrays have been used to identify protein substrates in vitro that are targeted by enzymes specific for a particular PTM. This approach still requires in vivo confirmation and is 
also not useful for the determination of multiple PTM sites ${ }^{11}$. Mass spectrometry has emerged as the premier method for primary protein sequence and PTM identification, because it offers the ability to directly detect multiple PTMs in complex samples on the femtomole - attomole level. Its continuing advancements are enabling researchers to study PTMs more effectively.

\subsection{Characterizing PTMs by Mass Spectrometry}

Major breakthroughs in the last 30 years have enabled mass spectrometry to be applied to the study of large biomolecules like peptides and proteins. Reducing it to its most basic components, a mass spectrometer consists of an ionization source, a mass analyzer, and a detector.

In the past, the most commonly used ionization methods were 'hard ionization' techniques like electron ionization (EI) and chemical ionization ( $\mathrm{Cl}$ ), whereby samples may be ionized by an electron beam or an ionized gas, respectively ${ }^{12}$. El is particularly energetic and can generally not be applied to thermally labile compounds or else they will completely decompose. $\mathrm{Cl}$ is gentler and more controllable but still generates ion fragments upon ionization. Both techniques are limited to the analysis of volatile, low-molecular weight compounds.

A major breakthrough for the mass spectrometric analysis of biomolecules came with the development of the 'soft ionization' technique, fast-atom bombardment (FAB) in $1981^{13}$. For the first time, peptides and other biomolecules up to 4000 Da could be routinely analyzed. FAB utilizes a beam of high-energy inert gas atoms to strike analyte dissolved in a liquid matrix to produce a secondary beam consisting of ionized analyte molecules. Two other ionization techniques were developed a few years later that further enhanced mass spectrometry of 
biomolecules. Matrix-assisted laser desorption/ionization (MALDI), developed simultaneously by Karas and Hillenkamp ${ }^{14}$, and Tanaka and co-workers ${ }^{15}$, is similar to FAB but utilizes a laser pulsed onto an improved analyte-matrix. MALDI improved upon the sensitivity and upper mass range of $\mathrm{FAB}$ as $<100 \mathrm{kDa}$ biomolecules could now be ionized ${ }^{16}$.

Electro-spray ionization (ESI), first introduced by Dole in $1968{ }^{17}$ and successfully coupled to mass spectrometry by Fenn and coworkers in $1984{ }^{18}$, arguably signifies the most major breakthrough in the analysis of biomolecules by mass spectrometry to date. This technique produces gas-phase ions from electrically charged liquid droplets by 'electrospraying' an analyte-containing solution at high voltage $(\sim 2 \mathrm{kV})$ and atmospheric pressure. ESI made two major advancements in bioanalytical mass spectrometry: (1) it offered compatibility with large, nonvolatile, thermally-labile biomolecules $>1$ MDa by imparting multiple charges to them while being simultaneously converted to the gas phase, and (2) it allowed continuous flow that has enabled researchers to couple ESI with separation techniques like liquid chromatography, enhancing the ability to analyze complex mixtures. Coupling high performance liquid chromatography (HPLC) with mass spectrometry is commonly referred to as LCMS, and represents the most popular mass spectrometric method for analyzing peptides and proteins today. The two major ionization models of ESI are the charged residue model (CRM) proposed by Dole ${ }^{17,19}$, and the ion evaporation model (IEM) proposed by Iribarne and Thomson ${ }^{20}$. The CRM model reasons that as charged liquid droplets become smaller by solvent evaporation, the charged particles in the droplet become closer and closer until the droplet reached its 'Rayleigh limit.' At this point, the coulombic repulsion forces exceed the surface tension of the droplet and it breaks apart into smaller droplets. This process continues until the eventual formation of 'ultimate droplets' that contain only one solute molecules and excess charge. As the remaining 
solvent evaporates, the solute molecule retains some of all of the charge to become a gas-phase analyte ion. According to IEM, electrospray droplets do not undergo fission at the 'Rayleigh limit' but rather the charge density at the surface becomes sufficient to force one or more charged solute ions into the ambient gas. This process repeats to continually 'bud' gas-phase analyte ions from the charged droplets. Numerous investigations of the ESI mechanism have led to conclusions that support both models, so it is plausible that a combination of both mechanisms occur in practice ${ }^{21}$.

Ion trap mass analyzers like the quadrupole linear ion trap (LTQ) work well in conjunction with LCMS. The multiply-charged biomolecules produced by ESI generally fall well within the mass-to-charge $(\mathrm{m} / \mathrm{z})$ range $(0-4000)$ of the $\mathrm{LTQ}$. For example, a typical 40kDa protein containing $\sim 350$ residues may be expected to contain an average of 1 charge per 10 residues. The monoisotopic $\mathrm{m} / \mathrm{z}$ of this protein would be $<1200$. The LTQ is an improvement upon the quadrupole ion trap (QIT), which like the LTQ, operated on the principle of trapping a population of ions and subsequently scanning them out according to their $\mathrm{m} / \mathrm{z}$ to acquire a mass spectrum. The LTQ effectively stretched the trapping area in the $z$ plane which increased ion space-charging limits before reductions in resolution and mass accuracy, isolation efficiency, activation efficiency, and storage capacity would occur. The LTQ is a sensitive mass analyzer that can acquire a mass spectrum of ions at femtomole-attomole levels with 1 unit $\mathrm{m} / \mathrm{z}$ resolution on a chromatographic timescale $(<100 \mathrm{~ms})^{22}$. A typical quadrupole consists of two pairs of opposing metal rods. In the LTQ, 12 hyperbolic poles form three distinct sections of the trap. Ions are trapped and analyzed according to quadrupolar theory using a combination of DC and RF potentials. The motion of ions through the LTQ is described by the Mathieu equation ${ }^{23}$, 
the solution of which contains the dimensionless parameters $a$ and $q$ that together describe the stability of an ion in the LTQ (Equation 1.1 \& 1.2).

(Equation 1.1) $a=\left(\frac{8 z U}{m \omega^{2} r_{0}^{2}}\right)$

(Equation 1.2) $q=\left(\frac{4 z V}{m \omega^{2} r_{0}^{2}}\right)$

Parameters $a$ and $q$ are directly proportional to the applied DC $(U)$ and RF $(V)$ potentials, respectively, where $z$ is the particular charge, $m$ is the particular mass, $\omega$ is the angular

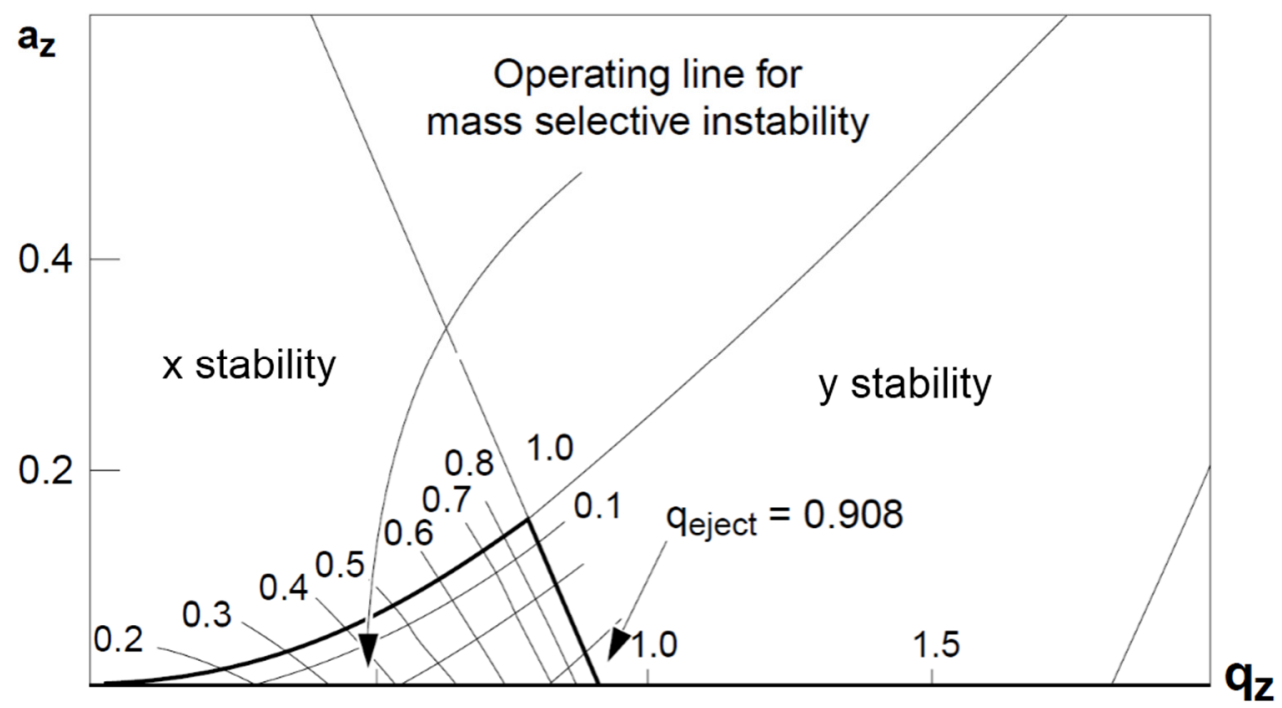

Figure 1.1 - The first stability region closest to the origin in which parameters $a$ and $q$ result in $x$ and y stability in the LTQ.

frequency, and $r_{0}$ is the radius between rods. The first stability region described by these parameters (Figure 1.1) is the most operational useful ${ }^{23}$. RF potentials applied to the rods keep the ions in stable trajectories in the $x$ and $y$ planes. The ions are axially ( $z$ plane) trapped by DC potentials applied on each of the three sections of the trap and/or the lenses on the front and back. Mass spectra are acquired using mass selective instability whereby RF amplitude applied to the rods is ramped upward, causing progressively higher $\mathrm{m} / \mathrm{z}$ values to adopt an unstable $q$ value along the $\mathrm{q}_{\mathrm{z}}$ axis and become ejected in the $\mathrm{x}$ plane. The ions exit through narrow slits on 
each $\mathrm{x}$-axis rod toward conversion dynodes where they are detected using electron multipliers that can achieve gains in excess of $10^{5}$, bolstering the sensitivity of the LTQ-based analysis ${ }^{23}$.

The speed and sensitivity of the LTQ make it ideal for quickly collecting a large number of spectra for many ions, even if they are in femtomole to attomole abundance. However, this speed can only be practically performed at $1 \mathrm{~m} / \mathrm{z}$ unit resolution which reduces mass accuracy and is not adequate to resolve isotopes. For this reason, LTQs are routinely placed in tandem with high-resolution mass analyzers like the FT-ICR and Orbitrap that may be routinely operated at resolutions $>100,000$ Full Width Half Max (FWHM) with mass accuracies of $<5$ parts-permillion (ppm).

The cylindrical ICR cell is situated inside a large magnetic field and a population of ions is injected and then trapped by front and rear trapping electrodes. The ions then receive a broadband excitation 'chirp' that sends them into a circular orbit perpendicularly to the magnetic field. The ions orbit at a cyclotron frequency that is proportional to their $\mathrm{m} / \mathrm{z}$ values. The image current of the orbiting ions is detected by two detector plates, simultaneously yielding amplitude and frequency data for all ions after translation by Fourier transform. ${ }^{23-25}$.

More recently, the Orbitrap has become the most popular high resolution mass analyzer for peptide and protein analyses as it does not require an expensive and high-maintenance superconducting magnetic to obtain high resolution mass spectra. Instead, ions are trapped around a central electrode using a purely electrostatic field. Ions are injected perpendicularly to and off-axis from the central electrode through a small inlet in the outer barrel-like electrode. They are electrostatically 'squeezed' closer to the central electrode by increasing the field strength and each $\mathrm{m} / \mathrm{z}$ ion packet will begin to rotate around the central electrode and also oscillate along the central electrode axis without any further stimulation needed. The frequency 
of axial oscillation is inversely proportional to the square root of $\mathrm{m} / \mathrm{z}$ and is independent of the spatial distribution and energy of the ions. Analogous to detection in the ICR cell, the ions' image current that contains amplitude and axial oscillation frequency information may be detected and converted into mass spectra by Fourier transform ${ }^{23,26-28}$. Taking advantage of the strengths of each mass analyzer in the LTQ-FT-ICR or LTQ-Orbitrap, it is common practice to acquire MS spectra of intact peptides or proteins in the high resolution analyzers and acquire MS/MS spectra for sequencing purposes in the LTQ.

Soft ionization through ESI enables the mass spectrometric analysis of proteins and peptides, but it does not offer any sequencing data due to a lack of fragmentation. Therefore, fragmentation techniques have been developed to randomly cleave peptides along their backbone to generate ladders of fragment ions differing in mass by each successive residue. In this way, the sequence of a peptide may be read in either direction by measuring the difference in mass between each successive ion fragment.

Collision-activated dissociation (CAD) is a common form of fragmentation in which the translational energy from multiple low-energy collisions between precursor ions and an inert bath gas (e.g. helium) is converted into vibrational energy imparted to the precursor ions. Random protonation of the amide nitrogens along the peptide backbone creates potential cleavage points. Once the energy from combinatorial collisions overcomes the necessary activation barrier, peptide bonds will be randomly cleaved to generate a series of complementary $\mathrm{b}$ and $\mathrm{y}$ ions, containing the $\mathrm{N}$-terminal and $\mathrm{C}$-terminal fragments of the peptide, respectively ${ }^{29}$ (Figure 1.2A\&B). 
(a)

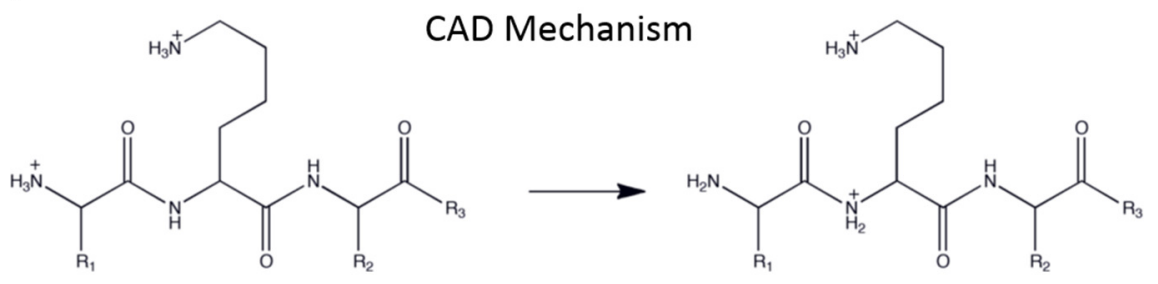

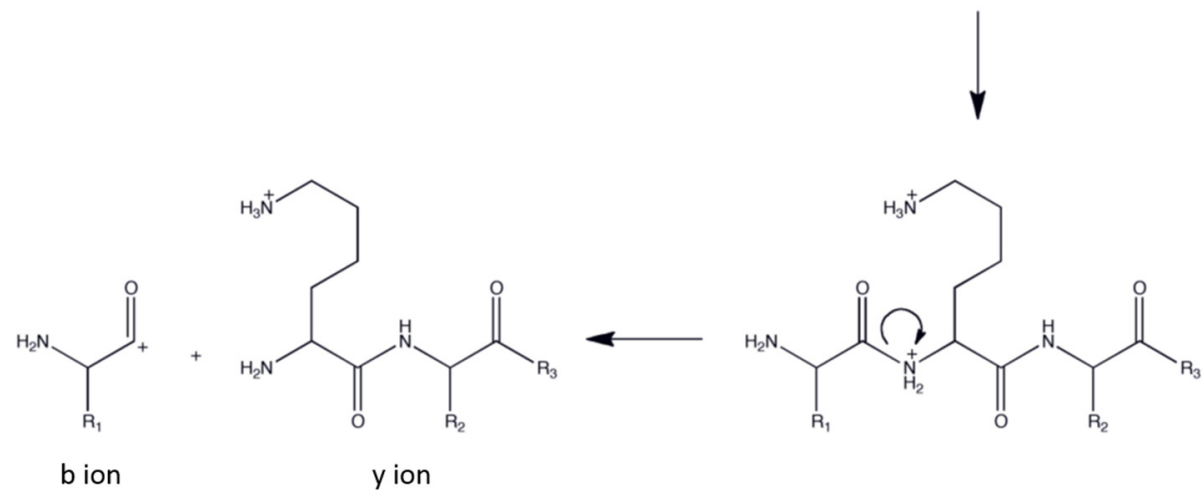

(b)

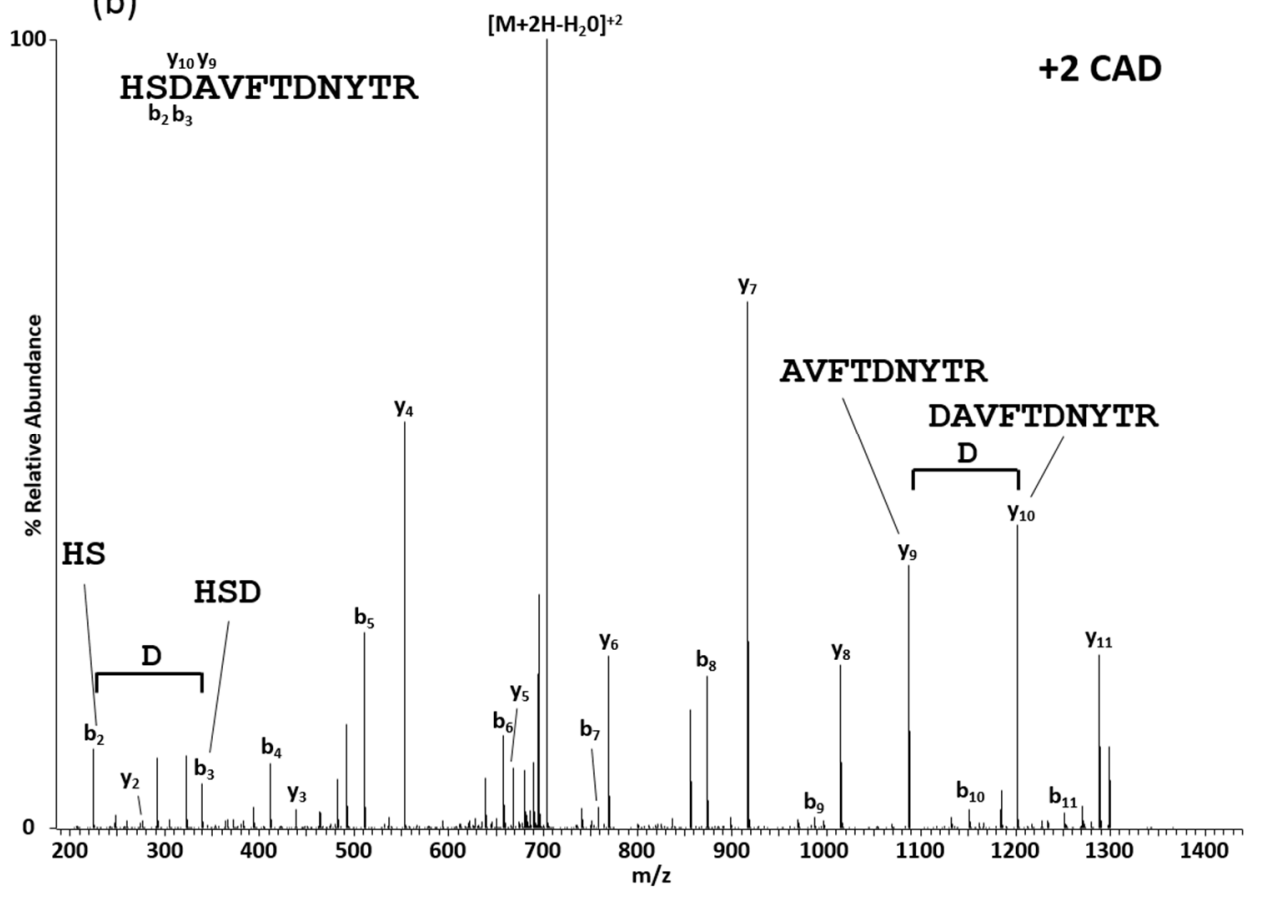

Figure 1.2 - (a) The fragmentation mechanism of CAD, and (b) a CAD MS/MS spectrum of peptide HSDAVFTDNYTR.

Unfortunately, ions with many charges and/or labile PTMs disrupt evenly distributed 
protonation and the cleavage of peptide bonds, making CAD much less useful for long peptides and those that contain phosphorylation and O-glycosylation.

To circumvent this problem, Hunt and coworkers developed electron transfer dissociation (ETD) ${ }^{30}$, an ion/ion analogue of electron-capture dissociation (ECD) ${ }^{31}$ which unlike ECD, can be performed in the LTQ. Fragmentation is induced after a radical anion reagent (e.g. azulene) transfers an electron to a random peptide carbonyl group, initiating a cascade based on free radical chemistry that induces fragmentation of $\mathrm{N}-\mathrm{C}_{\alpha}$ bonds along the peptide backbone, generating a series of $c$ and $z$ ions (Figure 1.3A\&B). ETD is complementary to CAD, because it provides the most optimal structural information for peptide ions of $z \geq 3$, and CAD is optimal for $z=2$ ions. Also, the ETD fragmentation mechanism is driven by the free radical electron vs.

(a)

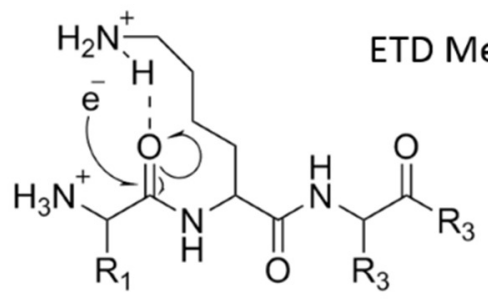<smiles></smiles>

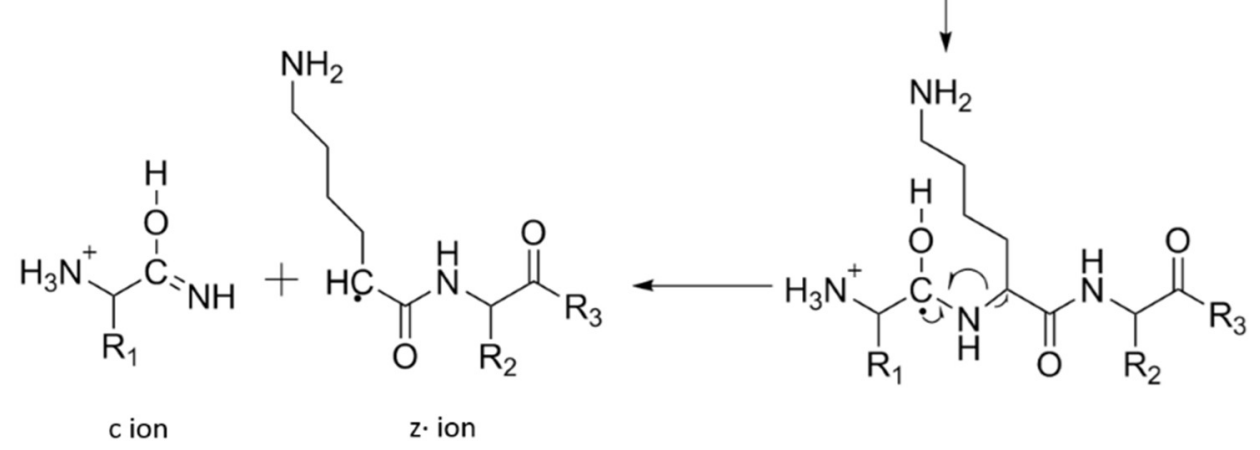


(b)

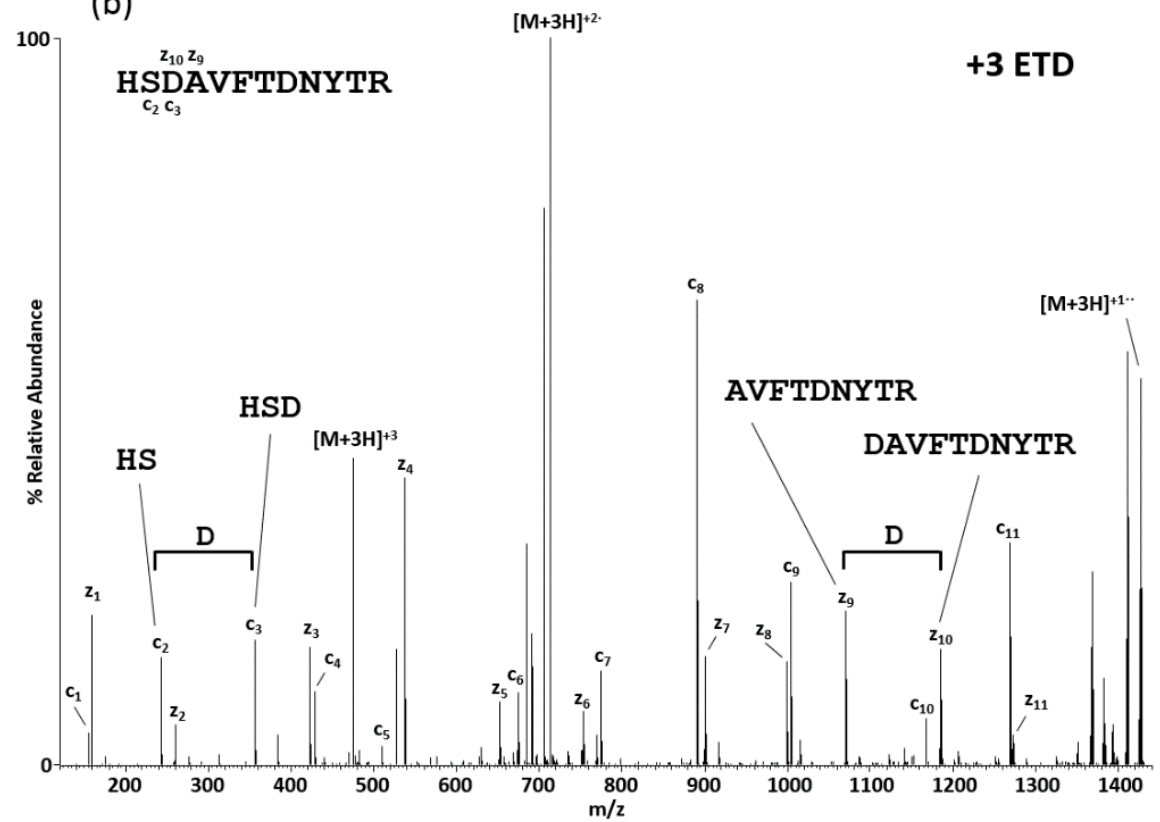

Figure 1.3 - (a) The fragmentation mechanism of ETD, and (b) an ETD MS/MS spectrum of peptide HSDAVFTDNYTR.

collisions with a bath gas, so in contrast to CAD, labile PTMs are preserved. Consequently, ETD is an essential MS/MS tool for detecting PTMs like phosphorylation and O-GlcNAcylation.

The following chapters describe the implementation of nanoflow micro-ESI LCMS using LTQ-FT-ICR and LTQ-Orbitrap mass spectrometers to characterize numerous novel PTMs on two nuclear proteins. Chapter 2 reports the post-translational modification of Arabidopsis protein repressor of ga1-3 (RGA), a negative regulator of plant growth, by O-GlcNAc, phosphate, and a 
Chapter 1

Introduction to the Dissertation

previously unreported O-linked hexose. Chapter 3 reports on the phosphorylation of

Saccharomyces cerevisiae DNA repair protein Rad9. 


\subsection{References}

1. CRICK, F. H. On protein synthesis. Symp. Soc. Exp. Biol. 12, 138-163 (1958).

2. Crick, F. Central dogma of molecular biology. Nature 227, 561-563 (1970).

3. http://www.ncbi.nlm.nih.gov/books/NBK26830/.

4. Cox, J. \& Mann, M. Quantitative, high-resolution proteomics for data-driven systems biology. Annu. Rev. Biochem. 80, 273-299 (2011).

5. Wilkins, M. R. et al. From proteins to proteomes: large scale protein identification by twodimensional electrophoresis and amino acid analysis. Biotechnology (N. Y) 14, 61-65 (1996).

6. International Human Genome Sequencing Consortium. Finishing the euchromatic sequence of the human genome. Nature 431, 931-945 (2004).

7. Walsh, C. T., Garneau-Tsodikova, S. \& Gatto, G. J.,Jr. Protein posttranslational modifications: the chemistry of proteome diversifications. Angew. Chem. Int. Ed Engl. 44, 7342-7372 (2005).

8. Creasy, D. M. \& Cottrell, J. S. Unimod: Protein modifications for mass spectrometry. Proteomics 4, 1534-1536 (2004).

9. Farley, A. R. \& Link, A. J. Identification and quantification of protein posttranslational modifications. Methods Enzymol. 463, 725-763 (2009).

10. Hart, G. W., Slawson, C., Ramirez-Correa, G. \& Lagerlof, O. Cross talk between OGIcNAcylation and phosphorylation: roles in signaling, transcription, and chronic disease. Annu. Rev. Biochem. 80, 825-858 (2011).

11. Zhao, Y. \& Jensen, O. N. Modification-specific proteomics: strategies for characterization of post-translational modifications using enrichment techniques. Proteomics 9, 4632-4641 (2009).

12. Ashcroft, A. E. \& Barnett, N. W. in Ionization Methods in Organic Mass Spectrometry X001X004 (The Royal Society of Chemistry, 1997).

13. Barber, M., Bordoli, R. S., Sedgwick, R. D. \& Tyler, A. N. Fast atom bombardment of solids as an ion source in mass spectrometry. Nature 293, 270-275 (1981).

14. Karas, M. \& Hillenkamp, F. Laser desorption ionization of proteins with molecular masses exceeding 10,000 daltons. Anal. Chem. 60, 2299-2301 (1988).

15. Tanaka, K. et al. Protein and polymer analyses up to $\mathrm{m} / \mathrm{z} 100000$ by laser ionization time-offlight mass spectrometry. Rapid Communications in Mass Spectrometry 2, 151-153 (1988).

16. Dass, C. in Fundamentals of Contemporary Mass Spectrometry 15-65 (John Wiley \& Sons, Inc., 2007; 2006).

17. Dole, M. et al. Molecular Beams of Macroions. J. Chem. Phys. 49, 2240-2249 (1968). 
Chapter 1

Introduction to the Dissertation

18. Whitehouse, C. M., Dreyer, R. N., Yamashita, M. \& Fenn, J. B. Electrospray interface for liquid chromatographs and mass spectrometers. Anal. Chem. 57, 675-679 (1985).

19. Mack, L. L., Kralik, P., Rheude, A. \& Dole, M. Molecular Beams of Macroions. II. J. Chem. Phys. 52, 4977-4986 (1970).

20. Iribarne, J. V. \& Thomson, B. A. On the evaporation of small ions from charged droplets. J. Chem. Phys. 64, 2287-2294 (1976).

21. Nguyen, S. \& Fenn, J. B. Gas-phase ions of solute species from charged droplets of solutions. Proc. Natl. Acad. Sci. U. S. A. 104, 1111-1117 (2007).

22. Schwartz, J. C., Senko, M. W. \& Syka, J. E. P. A two-dimensional quadrupole ion trap mass spectrometer. J. Am. Soc. Mass Spectrom. 13, 659-669 (2002).

23. Dass, C. in Fundamentals of Contemporary Mass Spectrometry 67-117 (John Wiley \& Sons, Inc., 2007; 2006).

24. Gross, M. L. \& Rempel, D. L. Fourier transform mass spectrometry. Science 226, 261-268 (1984).

25. Holliman, C. L., Rempel, D. L. \& Gross, M. L. Detection of high mass-to-charge ions by Fourier transform mass spectrometry. Mass Spectrom. Rev. 13, 105-132 (1994).

26. Makarov, A. Electrostatic axially harmonic orbital trapping: a high-performance technique of mass analysis. Anal. Chem. 72, 1156-1162 (2000).

27. Hardman, M. \& Makarov, A. A. Interfacing the orbitrap mass analyzer to an electrospray ion source. Anal. Chem. 75, 1699-1705 (2003).

28. Hu, Q. et al. The Orbitrap: a new mass spectrometer. J. Mass Spectrom. 40, 430-443 (2005).

29. Hunt, D. F., Zhu, N. Z. \& Shabanowitz, J. Oligopeptide sequence analysis by collision-activated dissociation of multiply charged ions. Rapid Commun. Mass Spectrom. 3, 122-124 (1989).

30. Syka, J. E., Coon, J. J., Schroeder, M. J., Shabanowitz, J. \& Hunt, D. F. Peptide and protein sequence analysis by electron transfer dissociation mass spectrometry. Proc. Natl. Acad. Sci. U. S. A. 101, 9528-9533 (2004).

31. Zubarev, R. A., Kelleher, N. L. \& McLafferty, F. W. Electron capture dissociation of multiply charged protein cations. A nonergodic process. J. Am. Chem. Soc. 120, 3265-3266 (1998). 


\section{Chapter 2: Characterization of O-GIcNAcylation, phosphorylation, and a novel O-linked nuclear PTM, O-hexose, on DELLA protein RGA}

\subsection{Introduction}

\subsubsection{Post-translational modification of proteins is ubiquitous and essential}

Post-translational modifications of proteins are essential for the cell to perform many biological functions. More than 400 PTMs have been discovered ${ }^{1}$, and this number could continue to increase as detection methods improve. A few of the most commonly studied PTMs include phosphorylation, methylation, acetylation, ubiquitination, sumoylation, and glycosylation. These and other PTMs regulate cellular function by covalent addition to proteins, the main biological components of cellular structure and function, thereby altering the proteins' physical and chemical properties. In this way, PTMs can influence protein activity, cellular location, and stability ${ }^{2}$.

The reversible phosphorylation of nuclear and cytoplasmic proteins was among the earliest PTMs discovered and it is one of the most ubiquitous PTMs known. There are $\sim 500$ human kinases, the proteins responsible for catalyzing protein phosphorylation from an ATP donor molecule, and nearly 150 human phosphatases, which catalyze hydrolytic phosphate removal ${ }^{3}$. It is estimated that $2-3 \%$ of all eukaryotic genes code for protein kinases ${ }^{4}$ and that $1 / 3$ of the proteome is phosphorylated ${ }^{5}$. Mammalian kinases modify nucleocytoplasmic proteins' Ser, Thr, and Tyr residues with phosphate to regulate most types of cellular function and their response to multiple environmental factors. This mediates cellular processes like proliferation, apoptosis, migration, RNA transcription, and protein translation. Consequently, dysregulation of protein kinases and phosphates is associated with many pathologies including cancer, autoimmune diseases, metabolic disorders, and infections ${ }^{6}$. The importance of 
phosphorylation makes it a primary focus for biochemical and proteomic studies. Yet, an analogous nucleocytoplasmic PTM, O-GIcNAc, was discovered less than 30 years ago and reveals an additional post-translational mechanism by which nuclear and cytoplasmic proteins are regulated in the cell.

\subsubsection{Post-translational modification O-linked $\beta$ - $\mathrm{N}$-acetylglucosamine}

O-linked $\beta$-N-acetylglucosamine, or O-GIcNAc, is a reversible monosaccharide PTM of nuclear and cytoplasmic proteins that forms an O-linkage to Ser and Thr, cycling on and off on a timescale similar to phosphorylation ${ }^{7}$. O-GlcNAcylation was discovered in 1984 when Hart and coworkers were probing for terminal GIcNAc moieties on the surface of human lymphocytes using $\left[{ }^{3} \mathrm{H}\right]$-galactose labeling. A portion of the radiolabel was covalently linked to intracellular proteins, and the signal could be removed by alkali-induced $\beta$-elimination, indicating O-linkage and revealing the existence of the nucleocytoplasmic PTM, O-GIcNAc ${ }^{8}$. Previously, it was widely believed that protein glycosylation was limited to the exterior of the cell and the luminal face of intracellular compartments. O-GlcNAcylation is a ubiquitous protein PTM in mammalian cells, with over 3000 O-GlcNAcylated proteins detected to date. Elucidating the scope and role of this glyco-PTM on nuclear and cytoplasmic proteins has been the focus of many biochemical and proteomic studies for the last 25 years.

O-GlcNAcylation differs from classic forms of protein glycosylation in several ways: (1) it modifies nuclear and cytoplasmic proteins rather than traditional glycosylation found on proteins localized to the extracellular membrane or matrix, lumen of organelles, or Golgi/ER, (2) it exists as a monosaccharide and is not elongated, contrary to the complex oligosaccharides lining the extracellular membrane, (3) O-GlcNAcylation is a dynamic modification, as opposed to static like most complex extracellular glycans, (4) O-GlcNAc forms an O-linkage to the oxygen 
atom on Ser and Thr but GlcNAc was previously known to form a direct bond to proteins only by an $\mathrm{N}$-linkage to the amide nitrogen on Asp, and (5) a consensus sequence for O-GlcNAc has not been determined while N-linked GlcNAcylation follows the consensus Asp-X-Ser/Thr (where X is any amino acid except Pro and Asp) ${ }^{9}$. In these ways, O-GlcNAc much more closely resembles phosphorylation. Both are dynamic nucleocytoplasmic protein PTMs, O-linked to Ser and Thr residues, usually substoichiometrically abundant, and are regulated by separate enzymes responsible for their addition and removal (Figure 2.1).
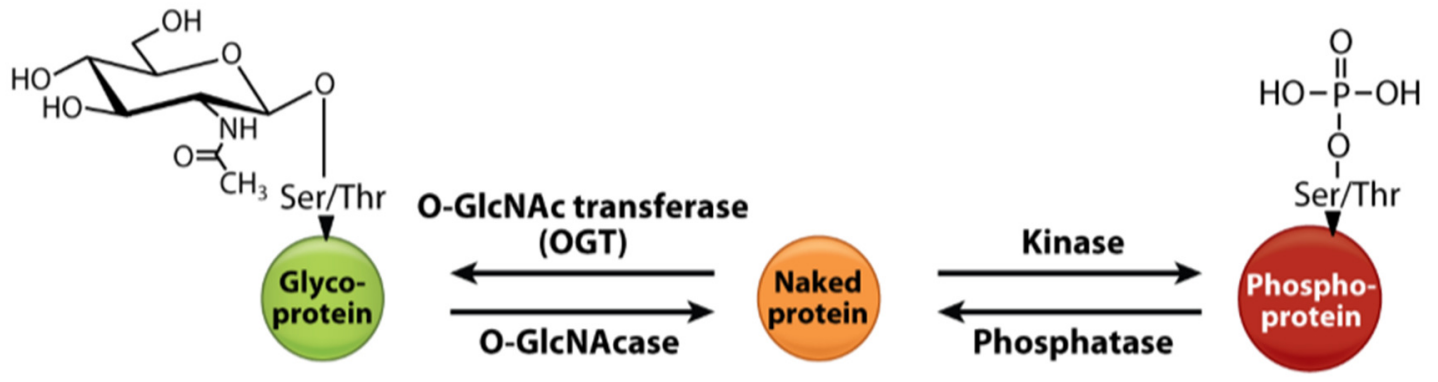

Figure 2.1-O-GlcNAc transferase and O-GlcNAcase respectively install and remove O-GlcNAc from Ser and Thr residues, analogous to the addition and removal of phosphate from these residues by kinases and phosphatases Figure reproduced from ${ }^{7}$.

However, in contrast to the many kinases and phosphatases responsible for phosphorylation addition and removal, just two metazoan genes encode the enzymes that cycle O-GlcNAcylation on and off Ser and Thr residues. These two respective enzymes are O-GlcNAc transferase (OGT) 


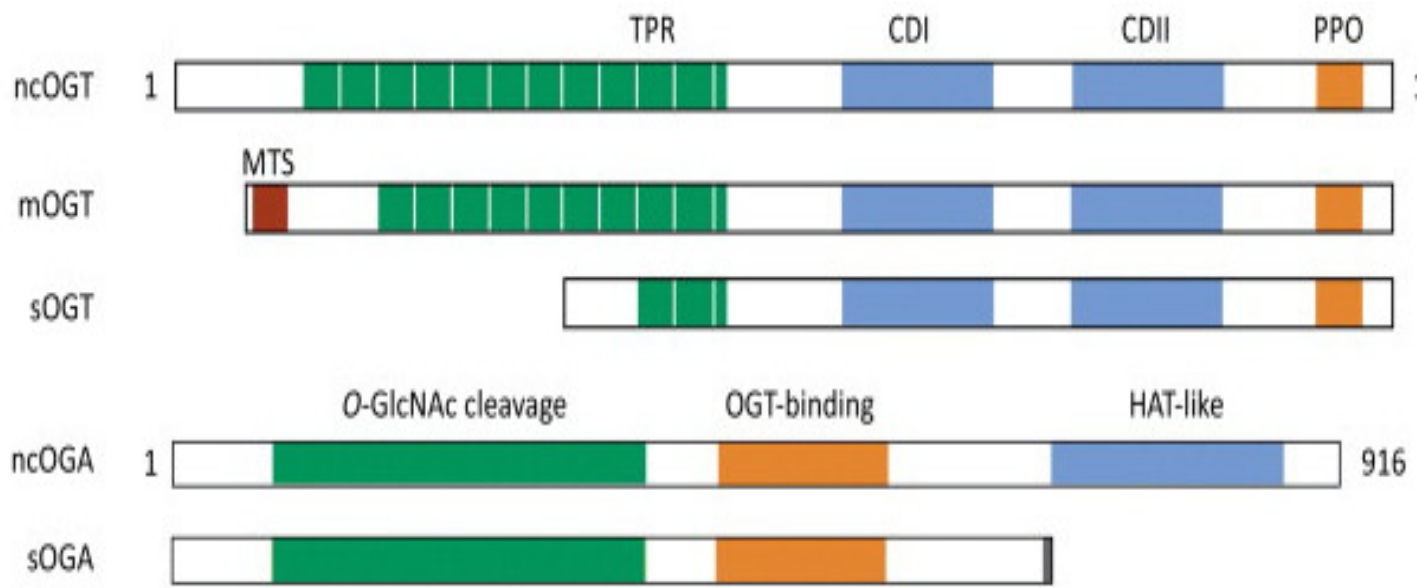

Figure 2.2 - O-GICNAc transferase (OGT) has three known human isoforms and O-GIcNAcase (OGA) has two, formed by alternative splicing and multiple start codons. The longest and shortest isoforms (ncOGT and sOGT) are found in the nucleus and the cytoplasm, and a third isoform (mOGT) targets the inner mitochondrial membrane form by an $\mathrm{N}$-terminal mitochondrial targeting sequence (MTS). Each OGT isoform has a unique number of $N$-terminal tetratricopeptide repeats (TPR), and a common C-terminal region containing catalytic domains I and II (CDI and II) in addition to a phosphoinositide-binding domain (PPO). The nucleocytplasmic and main form of OGA bears an N-terminal O-GlcNAc cleavage domain, an OGT-binding domain, and a C-terminal histone acetyltransferase (HAT)-like domain. A shorter HAT-like domain-deficient splice variant has been detected in the nucleus. Figure reproduced from ${ }^{10}$.

\section{O-GlcNAc transferase catalyzes the addition of O-GlcNAc to Ser and Thr}

OGT is essential in mammals. Loss of the OGT gene is embryonic lethal in mouse stem cells. OGT is ubiquitously expressed in all mammalian tissue, but is most abundant in the brain and pancreas. The single human OGT gene is located on the X chromosome. A full length 110 kDa OGT isoform (nucleocytoplasmic, ncOGT) and shorter 78-kDa isoform (sOGT), formed by a combination of alternative RNA splicing and start codons, both localize to the nucleus and cytoplasm, although they are more heavily localized in the nucleus ${ }^{11}$. They have been reported to form a heterotrimer composed of two ncOGT subunits and one sOGT subunit in rat liver, but most tissues including brain and pancreas, contain only the full length ncOGT isoform. A third form, mOGT, has been detected localizing to the inner membrane of mitochondria, and its 
function is less characterized ${ }^{12}$. One of the major characteristics of OGT is the multiple 34 residue tetratricopeptide repeats (TPRs) with the loosely conserved sequence, WLGYAFAP. Crystal structures demonstrate that each pair of TPRs form anti-parallel $\alpha$-helices that together form a super-helical structure important for protein-protein interaction ${ }^{13,14}$. Using its TPRs to form many different protein complexes is thought to provide the major means by which OGT is able to recognize and modify Ser and Thr residues on a wide variety of sequences. Several studies have collectively identified several OGT-interacting proteins. These include OGA which removes O-GlcNAc, Trak1 that recruits OGT to RNA polymerase II, and the arginine methyltransferase CARM $1{ }^{15}$. Overexpression of OGT alters the acetylation and methylation patterns of histones ${ }^{7}$.

O-GlcNAcase catalyzes the removal of O-GIcNAc from Ser and Thr

OGA, also known as Hexaminidase C, was identified on human chromosome 10 after the discovery of OGT and is responsible for the removal of O-GIcNAc from Ser and Thr residues ${ }^{16}$. OGA, like OGT, is also expressed by a single highly conserved gene among mammalian species, and homologs also exist in C. elegans, and Drosophila. OGA too localizes to the nucleus and cytoplasm, but contrary to OGT, the canonical full length isoform (ncOGA) is more concentrated in the cytoplasm. It contains the N-terminal catalytic domain, an OGT binding domain, and a Cterminal histone-acetyltransferase (HAT)-like domain ${ }^{16}$. The HAT-like domain is reported to demonstrate acetyltransferase activity in vitro on both all four core histones both free or DNAbound in the context of oligonucleosome arrays ${ }^{17}$. This activity was only observed when the OGlcNAcase was expressed in mammalian as opposed to bacterial cells. A shorter isoform (sOGA) which lacks the C-terminus and HAT-like domain has been reported to localize to the nucleus ${ }^{18}$. The sOGA isoform displays activity to O-GlcNAcylated proteins, but at a lesser rate vs. ncOGA 
(400-fold less efficient) ${ }^{19}$. Both OGA isoforms are subject to effective inhibition by small

molecule inhibiters like PUGNAc, NAG-thiazoline, and NButGT (Figure 2.3), implying similar catalytic reaction mechanisms. Much research is presently conducted toward the goal of
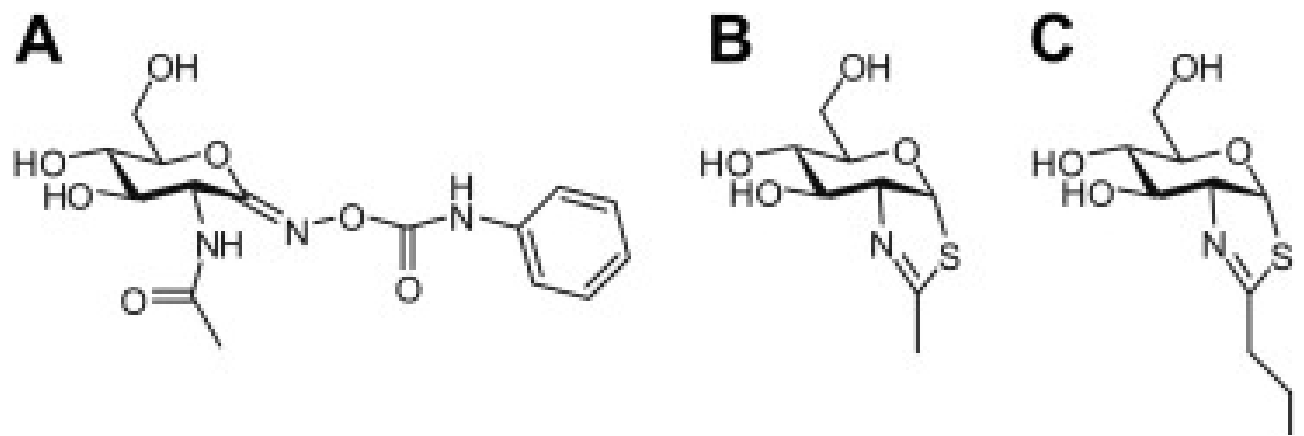

Figure 2.3 - Three OGA inhibitors, (A) PUGNAC, (B) NAG-thiazole, and (C) NButGT that effectively inhibited the nCOGA and SOGA isoforms. Figure reproduced from ${ }^{19}$. 
producing very effective and specific OGA inhibitors for the potential therapeutic benefit of controlling cellular OGA levels.

O-GICNAc donor molecule, UDP-GICNAc, is produced by the Hexosamine Biosynthetic Pathway

OGT has a relatively high binding affinity $(\mathrm{Km}=545 \mathrm{nM})$ for O-GlcNAc's donor molecule, UDP-GIcNAc, which is typically present in the cell at 0.1-1 mM. This molecule is produced by the Hexosamine Biosynthetic Pathway (Figure 2.4), a process tied to nearly every major metabolism pathway in the cell. OGT activity is dependent on UDP-levels from the low $\mathrm{nM}$ range to $>50 \mathrm{mM}$ 20. Accordingly, UDP-GIcNAc and therefore O-GIcNAcylation levels are sensitive to many factors such as insulin, nutrients, and cellular stress. For this reason, O-GlcNAcylation is believed to act as a "nutrient sensor", modulating cellular signaling and transcription regulatory pathways in response to these factors ${ }^{21-24}$.

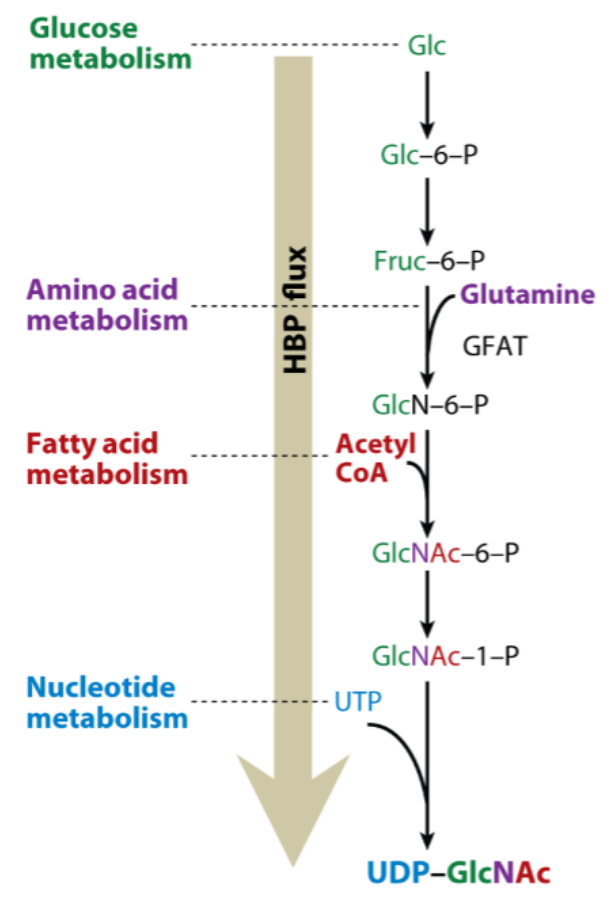

Figure 2.4 - All major forms of metabolism feed the Hexosamine Biosynthetic Pathway which produces the O-GICNAc donor molecule, UDP-GICNAC. Reproduced from 7. 

been found to be O-GlcNAcylated, regulating numerous processes that include transcription,

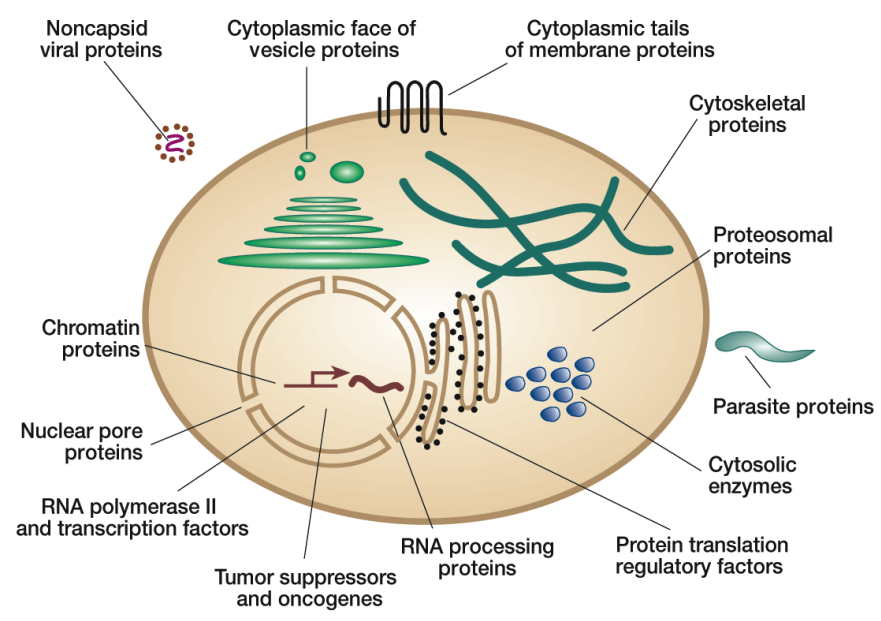

Figure 2.5 - Proteins from virtually every functional class are O-GIcNAcylated within the cell. Figure reproduced from ${ }^{20}$.

translation, protein trafficking, and protein degradation (Figure 2.6). Dysregulation of OGIcNAcylated proteins and altered cellular levels of O-GIcNAc and its regulating enzymes has negative implications for several pathologies: cancer, diabetes, neurodegenerative disease, and cardiovascular disease ${ }^{25}$. Transcription factors are amongst the most commonly O-

GlcNAcylated proteins identified. RNA polymerase II and most of its basal transcription factors are O-GlcNAcylated, in addition to other transcription factors including Sp1, estrogen receptors, STAT5, NF-kB, p53, YY1, Elf-1, c-Myc, Rb, PDX-1, and CREB ${ }^{20}$. The list of identified OGlcNAcylated proteins also includes many nuclear pore proteins, RNA-binding proteins, cytoskeletal proteins, and enzymes including kinases, phosphatases, and OGT itself. 


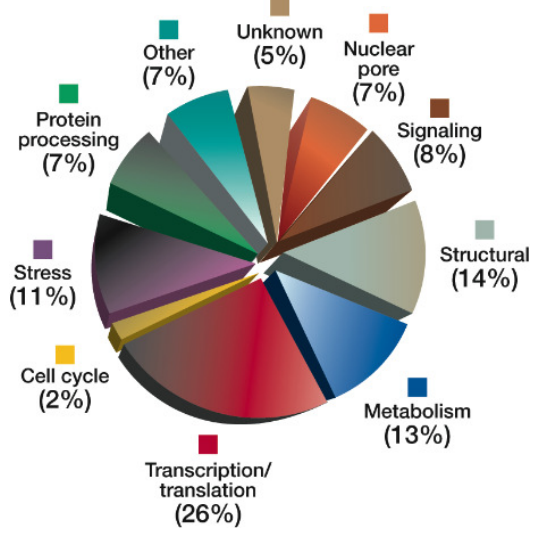

Figure 2.6 - The largest category of O-GlcNAcylated proteins are involved in transcriptional regulation, followed by those involved in cytoskeletal regulation and structure, energy metabolism, cellular stress, and signaling. Figure reproduced from ${ }^{20}$.

\section{Cross Talk between O-GlcNAcylation and phosphorylation}

Due to their many similarities, the relationship between O-GIcNAcylation and phosphorylation is of particular interest and is one of the most studied aspects of the glycoPTM. There are several mechanisms by which the two PTMs exhibit regulation over each other. As mentioned, OGT modifies numerous kinases and phosphates. Also, OGT and OGA may be tyrosine- and serine-phosphorylated, respectively, in their catalytic domains ${ }^{26}$. Notably, tyrosine phosphorylation by the insulin receptor has been shown to activate mammalian OGT, initiating a process by which OGT down-regulates insulin signaling ${ }^{27}$. OGT may also be activated by Src kinase and calcium calmodulin kinase IV (CAMKIV) amongst others ${ }^{28}$. Inhibition of kinase GSK3 $\beta$ increased O-GlcNAcylation on many cytoskeletal and heat shock proteins and decreased O-GIcNAc levels on many transcription factors and RNA-binding proteins ${ }^{29}$. Likewise, when OGlcNAcase is inhibited, overall O-GIcNAc levels increase three-fold, and nearly all 700 phosphorylation sites determined to be actively cycling in the cell demonstrated either increased or decreased when compared to (empty vector?) controls ${ }^{30}$. A large-scale enrichment of phosphorylated and O-GIcNAcylated proteins from M-phase HeLa cells following a two-fold 
overexpression of OGT during revealed over 400 O-GIcNAc modified peptides, comparable to the number of phosphorylated peptides present. ${ }^{31}$. OGT overexpression reduced the majority of phosphorylation sites, but a portion increased relative to basal levels. Ser and Thr residues on many cytoskeletal proteins were reciprocally modified with either PTM on the same site, while most transcription factors showed reciprocal occupancy of both PTMs on proximal sites.

\subsubsection{Evidence for O-GIcNAcylation in Plants}

Two OGTs are found in plants: SPINDLY and SECRET AGENT

Despite the increasing amount of knowledge being gained regarding metazoan OGlcNAcylation, the body of evidence for this PTM in other species, including plants, is much less developed. Still, two OGTs are now known to exist in plants, primarily through the study of Arabidopsis thaliana $^{32}$. The first to be discovered, SPINDLY (SPY), was originally identified as a gene locus important in gibberellin (GA) signaling ${ }^{33}$. GA's are plant growth hormones that regulate many aspects of plant development: germination, growth, flowering, and seed development ${ }^{34}$. Experiments with mutant spy alleles demonstrated that SPY negatively regulates GA signaling and plant development and also plays a role in circadian and light signaling ${ }^{33}$. When human OGT was discovered, researchers recognized the high homology of hOGT with SPY (Figure 2.7) and identified SPY as an O-GIcNAc transferase. A separate Arabidopsis OGT was later discovered and named SECRET AGENT ${ }^{32}$. The catalytic domain of SEC 


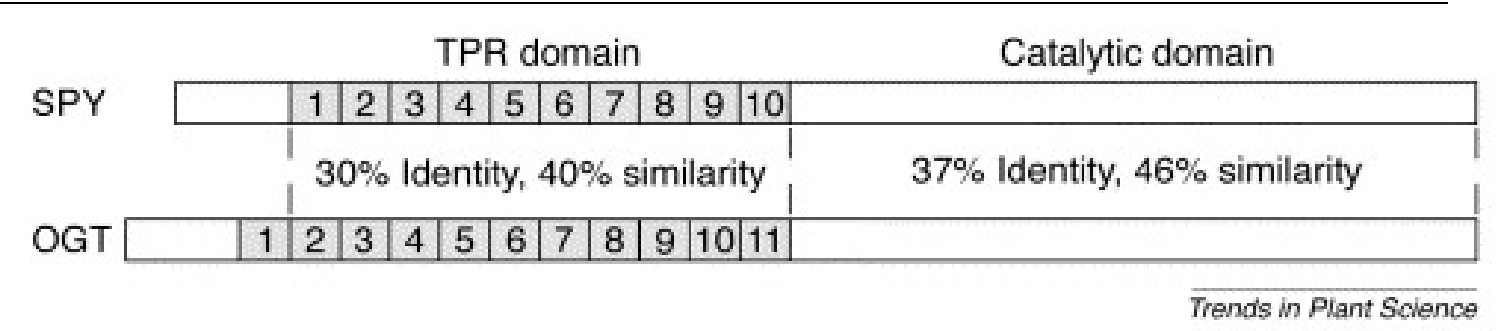

Figure 2.7 - Arabidopsis SPY (top) and human OGT (bottom) have high homology in both their TPR and catalytic domains. Reproduced from ${ }^{35}$.

is even more similar ( $>50 \%$ similarity) to animal OGT than SPY. In addition, SEC'S TPR domain is contiguous like that of animal OGT while SPY has amino acid insertions between the second and fifth TPRs. Direct evidence for O-GIcNAcylation by these OGTs is limited. Both appear to have the ability to auto-O-GIcNAcylate themselves, like animal OGT ${ }^{36}$. Direct mass spectral data demonstrates that several sites of the Plum Pox Virus capsid protein are O-GlcNAcylated, enhancing viral infection ${ }^{37,38}$. SEC has been shown to function in germination, rosette leaf growth/production rate, and inflorescence stem height, but evidence for its role in GA signaling is weak. However, the two OGTs appear to be at least partially functionally redundant. The loss of either was still viable though spy sec double knockout mutants were lethal in early development, indicating they are essential for gametogenesis and embryogenesis, similar to animal OGT ${ }^{32,39}$. It is possible that SPY has a greater ability to compensate for the loss of SEC than vice versa, making the effects of sec phenotypes more difficult to study.

Evidence for O-GICNAcylation of DELLA protein Repressor of ga1-3 (RGA)

The repressor of ga1-3 (RGA) is one of five Arabidopsis growth regulator proteins, characterized in part by their conserved N-terminal DELLA (named for the sequence Asp-GluLeu-Leu-Ala) and C-terminal GRAS (named after the first three isolated proteins $\underline{G} A l, \underline{R} G A$, and 
$\underline{\mathrm{S}} \mathrm{CR}$ ) domains ${ }^{40,41}$ (Figure 2.8).

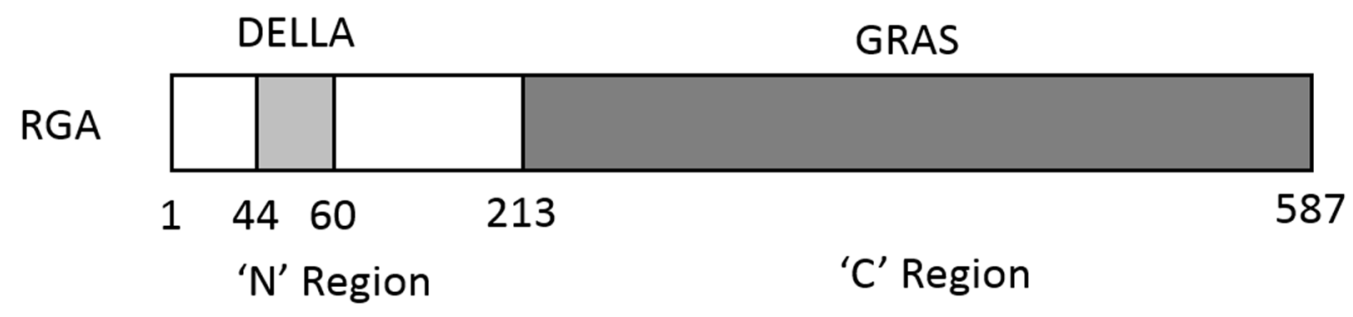

Figure 2.8 - A schematic of RGA is pictured with the DELLA domain (light gray) in the N-terminal region and the GRAS domain (dark gray) in the C-terminal. All five Arabidopsis DELLA orthologs are highly conserved.

The ga1-3 Arabidopsis mutant is the most studied mutant of the GA1 gene, whose loss of function abolishes GA biosynthesis ability ${ }^{40}$. Sun and coworkers performed screens of Arabidopsis proteins to find repressors of the mutant ga1-3 phenotype. Loss of function in RGA was found to repress the ga1-3 dwarf phenotype, partially restoring its ability to flower, and elongate its stem ${ }^{42}$. This demonstrated a similar phenotype to spy knockout mutants, already known to suppress development. Interestingly, rga spy double knockout mutants resulted in a virtually complete rescue of the ga1-3 dwarf phenotype (Figure 2.9). This could imply that OGT 


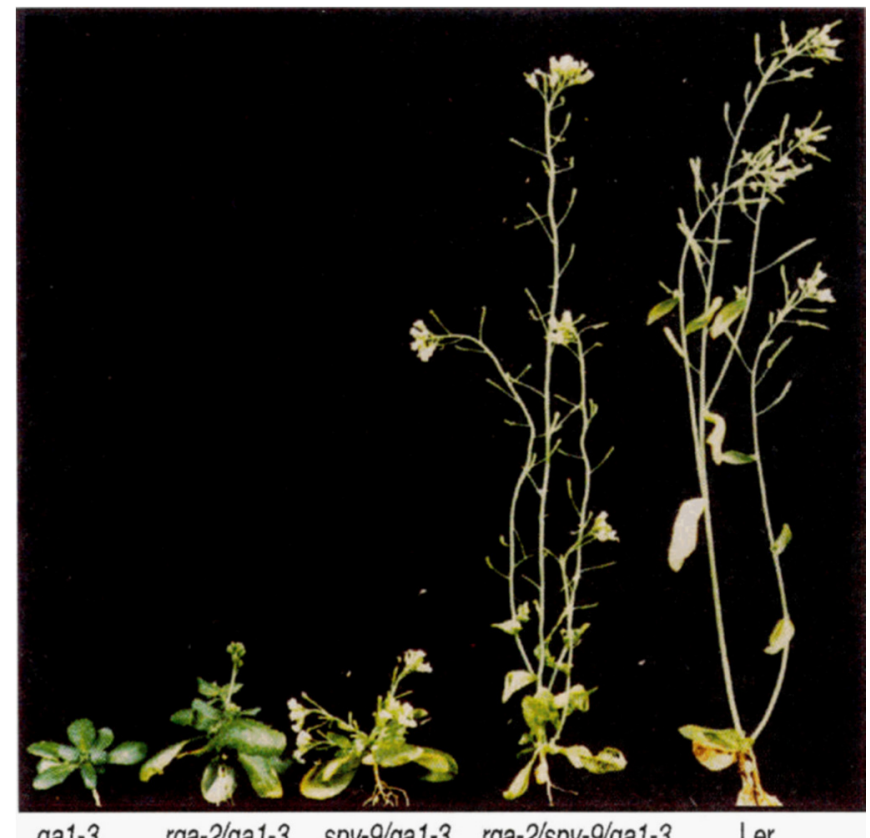

ga1-3 rga-2/ga1-3 spy-9/ga1-3 rga-2/spy-9/ga1-3 Ler

Figure 2.9 - 35-day-old mutant ga1-3 Arabidopsis plants lacking GA biosynthetic components necessary for proper growth (far left) were subject to additional mutation in the rga and/or spy locus (middle three). Wildtype (Ler) is displayed on the far right. DELLA protein RGA and OGT protein SPY each have a role in negatively regulating growth. Remarkably, rga spy double knockout mutants virtually restore ga1-3 to the Ler phenotype. Figure reproduced from ${ }^{42}$.

directly regulates RGA. Sun and coworkers also proposed that the two proteins each regulate separate but closely related branches of the GA signaling network with some overlapping functions ${ }^{42}$. Compared with the other DELLA proteins found in Arabidopsis, RGA appears to have a central role in developmental regulation, controlling germination, flowering, and stem elongation (Figure 2.10) ${ }^{43}$.

DELLA proteins do not have a canonical DNA binding motif, indicating that they regulate transcription through interactions with other transcription factors ${ }^{44}$. GA signaling is shown to promote plant growth by degradation of DELLAs. The mechanism by which this occurs has been 


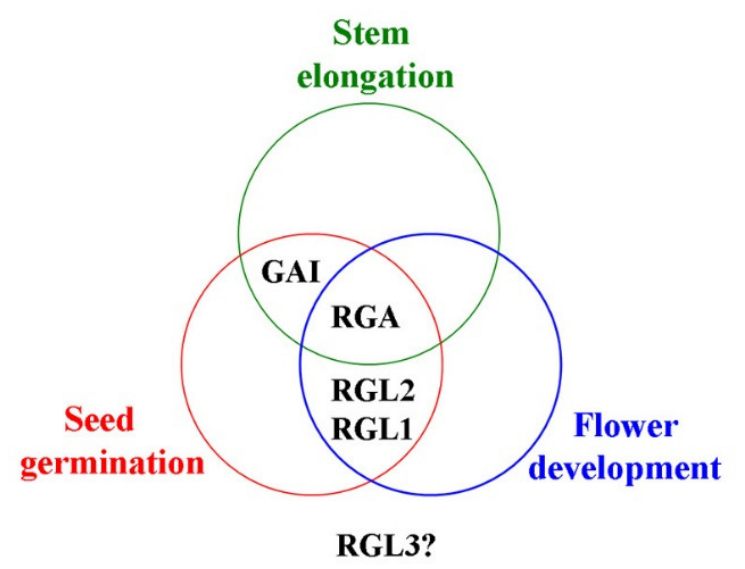

Figure 2.10 - Five DELLA proteins have been discovered in Arabidopsis. RGA plays a central role as a negative growth regulator. Figure reproduced from ${ }^{43}$.

recently studied and elucidated. Upon GA binding to the GA receptor Gibberellin Insensitive

Dwarf 1 (GID1), the affinity of the DELLA domain for GID1 also increases, causing it to bind GID1 as well.

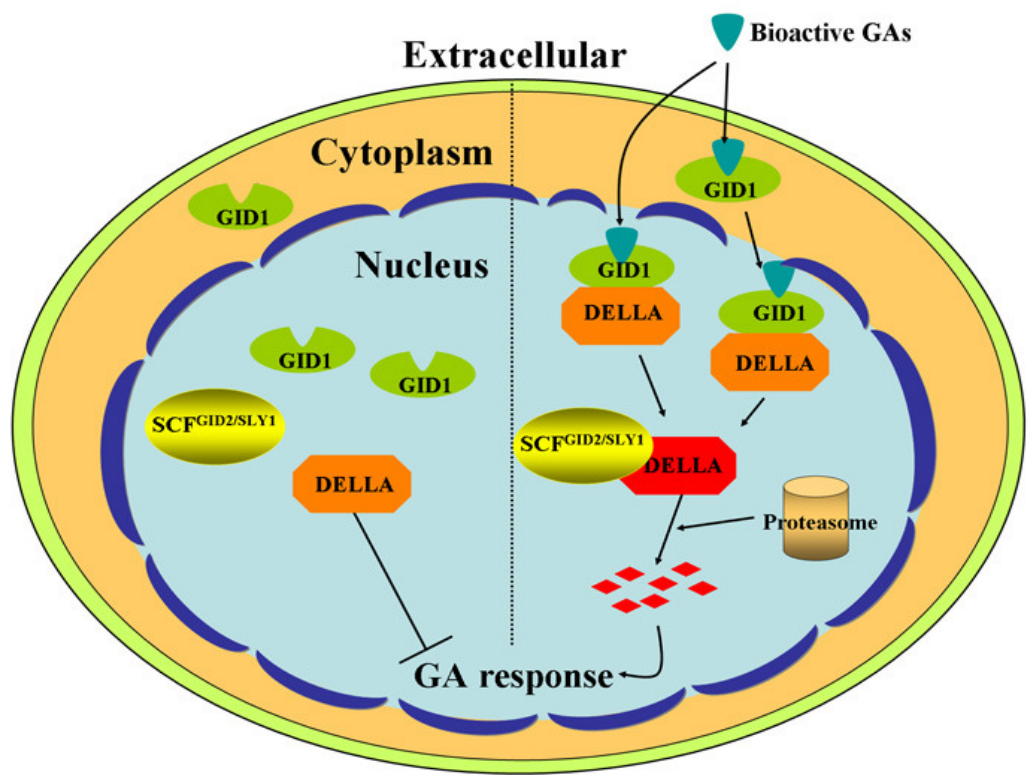

Figure 2.11 - GAs are reported to stimulate plant development by inducing degradation of the repressor DELLA proteins. Figure reproduced from ${ }^{43}$. 
This recruits E3 ubiquitin ligase to mark DELLA for degradation through the Skp, Cullin, F-box containing (SCF) complex, using F-box protein SLEEPY1 (SLY1) (Figure 2.11).

No direct evidence exists for the post-translation modification of RGA. Neither has any Arabidopsis or any other plant protein been directly characterized for O-GIcNAcylation by SPY or SEC. To enable a more complete understanding of the mechanisms by which RGA and OGT act to regulate plant growth and GA signaling down-regulates RGA, this study aims to fully characterize RGA for O-GIcNAcylation and other PTMs by mass spectrometry.

\subsection{Materials and Methods}

\subsubsection{Proteolytic Digestions and Sample Preparation for Mass Spectrometric Analysis}

Seven separate preparations of 6xHis-3xFLAG-tagged Arabidopsis RGA overexpressed in tobacco were tandem-purified (10-50 pmol each) and reduced on anti-FLAG M2 agarose beads (Sigma) (1 hour) with dithiothreitol (DTT; Sigma) at a molar ratio of 20: 1 (DTT: cysteine), carbamidomethylated (45 min) with iodoacetamide (IAA; Sigma) at a molar ratio of 3: 1 (IAA: DTT), digested with trypsin (Promega), endoproteinase LysC (Roche), or endoproteinase AspN (Roche) (7 hours) at a molar ratio of 20: 1 (RGA: protease), then quenched with glacial acetic acid to $\mathrm{pH}$ 3-4 for storage at $-35^{\circ} \mathrm{C}$. In four preparations, Arabidopsis O-GIcNAc transferase SEC was co-overexpressed with WT Arabidopsis RGA. In a fifth preparation, Arabidopsis SEC was cooverexpressed with Arabidopsis RGA containing a lysine insertion between G185 and G186 (G185_G186insK). In a sixth preparation, Arabidopsis kinase CK1 was co-overexpressed with Arabidopsis RGA-G185_G186insK. In a seventh preparation, ArabidopsisRGA-G185_G186insK was overexpressed in the absence of SEC or CK1. Reduction, alkylation, and digestion were carried out at room temperature in volumes $\leq 100 \mathrm{uL} 100 \mathrm{mM}$ ammonium bicarbonate $\mathrm{pH}$ 7.9. 


\subsubsection{Subcleavage by $\mathrm{CNBr}$}

A subset of AspN-digested WT RGA was cleaved C-terminally to Met using cyanogen bromide (CNBr). The reaction was performed as described by ${ }^{1}$ with slight adjustments. Peptides were dissolved in 20uL 70\% TFA in water $(v / v)$, one small crystal $<1 \mathrm{mg}$ of $\mathrm{CNBr}$ was added to the solution (a large excess over total Met), and the reaction proceeded 16 hours at room temperature. Following cleavage, the peptides were dried down and resuspended in $0.1 \%$ acetic acid.

\subsubsection{Derivatization of Carboxylic Acids with Histamine}

Peptides' carboxylic acid groups were derivatized with histamine as described in ${ }^{45}$. Peptides were first purified from proteases and desalted by loading $(<1 \mathrm{uL} / \mathrm{min})$ onto a $360 \mu \mathrm{m}$ outer diameter (o.d.) $\times 75 \mu \mathrm{m}$ inner diameter (i.d.) fused silica (Polymicro Technologies, LLC) column packed with $6 \mathrm{~cm}$ of C18 reverse-phase resin (5-20 $\mu \mathrm{m}$ diameter, $120 \AA ̊$ pore size, YMC Co., Ltd.) behind a 1-2mm Kasil ${ }^{\circledR} 1624$ (PQ Corporation) frit. The column was rinsed with $0.1 \mathrm{M}$ acetic acid for 20 min then peptides were gradient eluted into a clean Eppendorf tube for 70 min using an LC gradient of $0-80 \%$ Solvent B in $60 \mathrm{~min}$, hold $80 \%$ Solvent B for $30 \mathrm{~min}, 80-100 \%$ Solvent B in 10 min, 100\%-0\% Solvent B in 5 min, 100\% Solvent A for 20 min (Solvent A: $0.1 \mathrm{M}$ acetic acid in water and Solvent B: $70 \%$ acetonitrile in $0.1 \mathrm{M}$ acetic acid in water). Peptides were dried down and resuspended in $20 \mathrm{uL} 1 \mathrm{M}$ histamine in $1 \mathrm{M}$ pyridine- $\mathrm{HCl}$ and $5 \mathrm{uL} 0.1 \mathrm{M}$ EDC in 1 $\mathrm{M}$ pyridine- $\mathrm{HCl}$. The reaction was sonicated for 2 hours at room temperature then left to proceed without sonication for 14 hours. Following reaction, peptides were loaded directly onto the pre-column. 


\subsubsection{HPLC Capillary Analytical and Pre-column Assembly}

Pre-columns were constructed from $8 \mathrm{~cm}$ of $360 \mu \mathrm{m}$ o.d. $\times 75 \mu \mathrm{m}$ i.d. fused silica and packed with $4 \mathrm{~cm}$ of C18 reverse-phase resin (5-20 $\mu \mathrm{m}$ diameter, $120 \AA ̊$ pore size, YMC Co., Ltd.) behind a 1-2mm Kasil ${ }^{\circledR} 1624$ (PQ Corporation) frit. Analytical columns were constructed from 10 $\mathrm{cm}$ of $360 \mu \mathrm{m}$ o.d. $\times 50 \mu \mathrm{m}$ i.d. fused silica and packed with $7 \mathrm{~cm}$ of C18 resin $(5 \mu \mathrm{m}$ diameter, $120 \AA ̊$ pore size, YMC Co., Ltd.) behind a Lithisil $^{\circledR}$ (PQ Corporation) frit, prior to being equipped with a laser-pulled electrospray emitter tip as previously described ${ }^{3}$. Columns packed with C4/C8 (3 $\mu \mathrm{m}$ diameter, $120 \AA ̊$ pore size, YMC Co., Ltd.), Zirchrome ${ }^{\circledR}$-CARB, and POROS ${ }^{\circledR}$ R2 were prepared in the same manner. Tens of picomoles of standard peptide Angiotensin I (Sigma) were loaded and eluted from columns to optimize their chromatographic resolution and sensitivity by occupying non-specific binding regions before use on RGA samples.

\subsubsection{Sample loading and LC Methodology}

Prior to mass spectrometric analysis, each digest was dried down and resuspended in $0.1 \%$ acetic acid $(\mathrm{pH} 3)$ in water $(\mathrm{v} / \mathrm{v})$. Typically, a $0.5-1 \mathrm{pmol}$ fraction of sample $+100 \mathrm{fmol}$ of internal standard peptides Angiotensin I (Sigma) and Vasoactive Intestinal Peptide fragment 112 (Sigma) in a volume $\leq 10 \mathrm{uL}$ was pressure loaded onto a pre-column at a flow rate of $<1 \mu \mathrm{L} / \mathrm{min}$ followed by a $15 \mathrm{~min}$. desalting rinse with $0.1 \mathrm{M}$ acetic acid at several $\mu \mathrm{L} / \mathrm{min}$. The pre-column was butt-connected to the analytical column with a $2 \mathrm{~cm}$ Teflon sleeve $(0.060 \mathrm{in}$ o.d. x 0.012 in i.d.; Zeus Industrial Products). Trypsin-, AspN-, or AspN/CNBr-generated peptides were gradient eluted and electrosprayed into the mass spectrometer at a split-flow-generated rate of $60 \mathrm{~nL} / \mathrm{min}$ by an 1100 series binary LC pump (Agilent Technologies) using a linear LC gradient of $0-60 \%$ Solvent B in $60 \mathrm{~min}, 60-100 \%$ Solvent B in $4 \mathrm{~min}$, hold $100 \%$ Solvent B for 4 
min, 100\%-0\% Solvent B in $4 \mathrm{~min}, 100 \%$ Solvent A for $20 \mathrm{~min}$ (Solvent A: 0.1M acetic acid in water and Solvent B: $70 \%$ acetonitrile in $0.1 \mathrm{M}$ acetic acid in water).

\subsubsection{Phosphorylated Peptide Enrichment by Immobilized Metal Affinity Chromatography}

A 5 pmol portion each of AspN- and trypsin-digested RGA-WT(+SEC) were combined and enriched for phosphorylation by Immobilized Metal Affinity Chromatography (IMAC). In addition, 5 pmol of AspN- and trypsin-digested RGA-G185_G186insK (+CK1) were separately enrichment by IMAC. Prior to enrichment, carboxylic acids were blocked by esterification with methanol to prevent them from preferentially binding to the IMAC column. Peptides were dissolved in 80 uL of a 6.25: 1 acetyl chloride: anhydrous methanol mixture that had been stirred for $5 \mathrm{~min}$, the peptides and reaction mixture were allowed to stand $1 \mathrm{hr}$, dried down, and the process was repeated. Esterified samples were stored at $-35^{\circ} \mathrm{C}$ for $<24 \mathrm{hrs}$ until enrichment by IMAC.

An $8 \mathrm{~cm} 360 \mu \mathrm{m}$ o.d. $\times 100 \mu \mathrm{m}$ i.d. fused silica column equipped with a $1 \mathrm{~mm}$ Kasil $^{\circledR} 1624$ frit was packed with $5 \mathrm{~cm}$ of POROS ${ }^{\circledR}$ MC 20 beads ( 20 uM diameter; Applied Biosystems ${ }^{\mathrm{TM}}$ ). The IMAC column was washed for $10 \mathrm{~min}$ with the following at $20 \mu \mathrm{L} / \mathrm{min}$ in this order: water, $50 \mathrm{mM}$ EDTA ( $\mathrm{pH} 9)$, and water. The column was activated $3 x$ with $100 \mathrm{mM} \mathrm{FeCl} 3$ for $10 \mathrm{~min}$ at 20 $\mu \mathrm{L} / \mathrm{min}$. Between each round of activation, the $\mathrm{FeCl}_{3}$ was allowed to sit for 3 min on column without flow. Excess $\mathrm{FeCl}_{3}$ was removed by washing with $25 \mu \mathrm{L} 0.01 \%$ acetic acid at $<1 \mu \mathrm{L} / \mathrm{min}$.

Peptide samples were reconstituted in a 1: 1: 1 mixture of $0.01 \%$ acetic acid: acetonitrile: methanol, then loaded onto the IMAC column at $<1 \mu \mathrm{L} / \mathrm{min}$. The 1: $1: 1$ mix was added to the empty sample tube again and the loading process repeated. The IMAC column was washed with $20 \mu \mathrm{L}$ of 1: 1: $1 \mathrm{mix}$, followed by $20 \mu \mathrm{L}$ of $0.01 \%$ acetic acid at $<1 \mu \mathrm{L} / \mathrm{min}$. The IMAC 
column was connected to a pre-column, constructed as previously described, and enriched phosphorylated peptides were eluted and captured on the pre-column using $15 \mu \mathrm{L} 250 \mathrm{mM}$ ascorbic acid at $1 \mu \mathrm{L} / \mathrm{min}$. Combined columns were washed with $6 \mu \mathrm{L} 0.1 \%$ acetic acid, the precolumn was removed, and the wash was repeated on the pre-column only at $20 \mu \mathrm{L} / \mathrm{min}$. The pre-column was connected to an analytical column, $100 \mathrm{fmol}$ of Angiotensin and Vasoactive Intestinal Peptide fragment $1-12$ were loaded onto the columns in $0.1 \%$ acetic acid, and LCMS analysis was conducted as described.

\subsubsection{Mass Spectrometric Analysis}

Mass analysis was completed with a modified ETD-enabled high-resolution LTQ-FT or LTQ-Orbitrap mass spectrometer (Thermo Scientific) using an acquisition method consisting of one high resolution $\mathrm{MS}^{1}$ scan $\{$ resolving power of 50,000 (FT Ultra, Thermo Scientific) or 60,000 (Orbitrap, Thermo Scientific) at $\mathrm{m} / \mathrm{z} 400$ \} acquired in the FT-ICR or Orbitrap followed by 8-10 collision-activated dissociation (CAD) and/or electron transfer dissociation (ETD) $\mathrm{MS}^{2}$ scans acquired with the LTQ operating in either the data-dependent or -independent mode. Datadependent parameters included a repeat count of 2-3, repeat duration of 20-30 s, and exclusion list duration of 20-30 s. $\mathrm{MS}^{2}$ parameters for ETD scans included $30-50 \mathrm{~ms}$ reaction times, $3 \mathrm{~m} / \mathrm{z}$ precursor isolation window, charge state rejection "on" for +1 (and +2 for ETD only analyses) and unassigned charge state precursor ions, $5 \times 10^{5}$ FTMS (Orbitrap) or $1 \times 10^{6}$ (FT Ultra) full automated gain control target, $1 \times 10^{4} \mathrm{ITMS}^{\mathrm{n}}\left(\mathrm{CAD}\right.$ and CAD/ETD toggle) or $6 \times 10^{4}$ automated gain control target (ETD), and $2 \times 10^{5}$ reagent target with azulene as the electron transfer reagent. ETD was employed for all PTM site identifications on peptides bearing $\geq 2$ modifiable residues. CAD and ETD date-independent targeting modes were employed for site-mapping or 
acquired. Database analysis was performed using The Open Mass Spectrometry Search

Algorithm (OMSSA), version 2.1.1., to search (parameters: \pm 0.01 Da precursor mass tolerance, \pm 0.35 Da fragment ion mass tolerance) against the RGA sequence ( $\mathrm{N}$-terminally His-FLAG-tagged RGA Uniprot Accession Q9SLH3). Either "Trypsin", "AspN", or "No enzyme" were used as protease options, with up to 3 missed cleavages allowed when applicable. Specified variable modifications were carbamidomethylation of Cys, oxidation of Met, O-GlcNAcylation of Ser \& Thr, Phosphorylation of Ser, Thr, \& Tyr, and O-hexosylation of Ser \& Thr residues. Search parameters also included variable modifications of Met to homoserine and homoserine lactone after $\mathrm{CNBr}$ cleavage, and acid amidation with histamine on Asp, Glu, and C-terminal residues when applicable. Signals corresponding to charge-reduced species were removed from the spectra by OMSSA before the database was searched. While OMSSA searches were used as a guide, all PTM site identifications were validated by manual interpretation of raw data.

\subsection{Results and Discussion}

\subsubsection{6xHis-3xFlag-tagged RGA Purification}

Tobacco plants were used as an expression system to generate highly O-GIcNAcylated RGA protein amenable to MS analysis. Tobacco plants were transfected by Agrobacterium to over-express Arabidopsis Thaliana 6x His- and 3x FLAG-tagged protein RGA and Arabidopsis Thaliana O-GICNAc transferase proteins SEC or SPY. These two N-terminal affinity tags allowed for a tandem purification method that firstly took advantage of the affinity of histidine residues for $\mathrm{Ni}^{2+}-\mathrm{NTA}$ resin ${ }^{46}$, then secondly utilized an antibody-antigen interaction using the 22 amino acid 3xFLAG tag, a variation of the original 8 amino acid FLAG sequence ${ }^{47}$. This two-dimensional purification minimized non-RGA protein contamination while yielding pmols of RGA bound to anti-FLAG antibody on agarose beads, amenable for on-beads proteolytic digestion and analysis 
by MS. RGA co-expressed with SEC was characterized by a gel shift of 2-3 kDa, implying it was

heavily O-GlcNAcylated (Figure 2.12). When select poly-Ser/Thr regions were mutated to alanine, the shift was nearly absent as visualized by Western Blot (Figure 2.13). RGA coexpressed with SEC was analyzed by mass spectrometry to fully characterize putative RGA(+SEC) O-GIcNAcylation and any other post-translational modification.

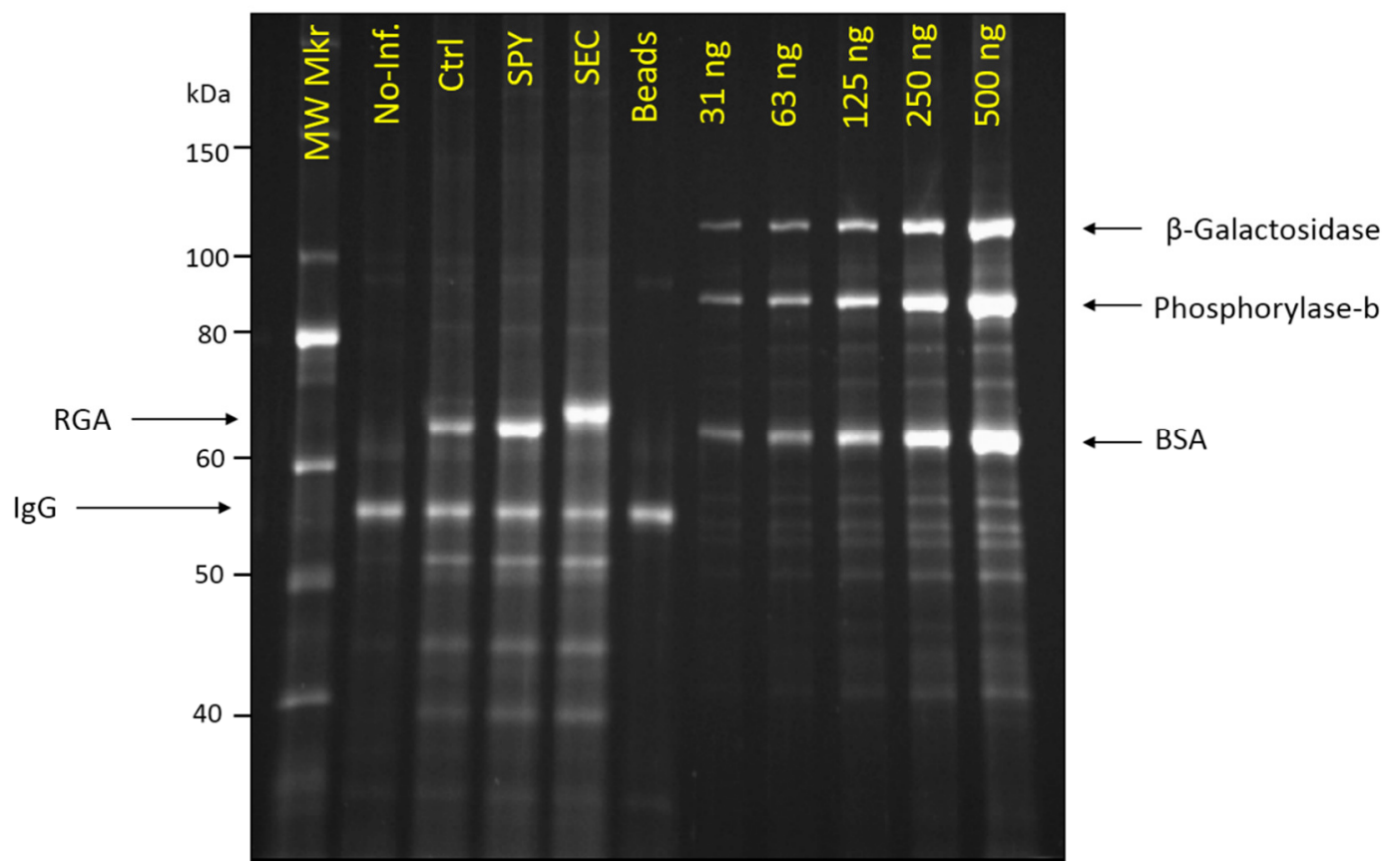

Figure 2.12 - RGA visualized by Coomassie Brilliant Blue stain on a 6\% acrylamide SDS-PAGE gel. MW Mkr - Molecular weight marker, No-Inf. - Non-transfected Tobacco leaf extract tandem purified, Ctrl-RGA expressed without OGT, SPY - RGA coexpressed with SPY, SEC - RGA coexpressed with SEC, Beads - Anti-FLAG agarose beads control, 31-500ng - three standard proteins used for abundance curve. 


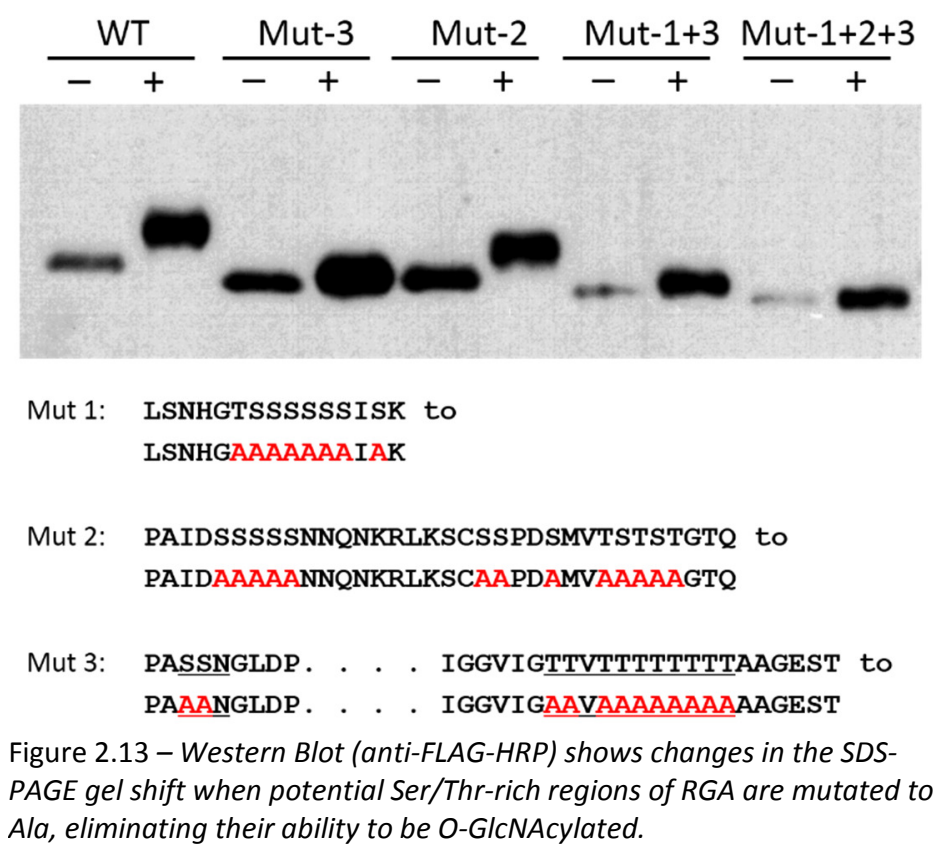

\subsubsection{In Silico RGA Digestion}

Protein mass spectrometrists generally regard trypsin as their protease of choice, because it efficiently cleaves peptides C-terminal to Lys and Arg residues with high fidelity ${ }^{48}$. This produces peptides well-suited for (i) separation by octadecyl carbon chain (C18) reversephase chromatography due to their ideal size and charge state upon ESI (typically $z=+2$ and $<15$ residues) and (ii) acquiescence for CAD MS/MS whereby protonated amide nitrogens facilitate the collision-activated cleavage of peptide bonds to generate a series containing every possible $\mathrm{b}$ and $\mathrm{y}$ fragment ion. Furthermore, the presence of one charge originating from the $\mathrm{N}$-terminal amine and another from the C-terminal Lys or Arg residue respectively allows for the detection of each resulting singly-charged $(z=+1) b$ and $y$ ion. In this way, a peptide's amino acid sequence along with any non-labile post-translational modifications can be read forwards and backwards by determining the mass differences between each successive fragment. Still, relying 
solely upon this trypsin digest and CAD approach has two major disadvantages: (i) it is

uncommon to achieve detection of a protein's entire sequence using one protease, and the

predominately doubly-charged $(z=+2)$ peptides typically generated from a tryptic digest are not amenable to ETD, the fragmentation method of choice for site-mapping labile O-linked PTMs like O-GIcNAc ${ }^{49}$.

In silico digestions of RGA were performed to determine candidate peptides that would allow for the detection of all Ser/Thr residues and produce quality fragmentation by ETD. Two separate theoretical digests using Endoproteinase AspN and trypsin met this goal by generating peptides of $\sim 10-40$ amino acids in length that were predicted to result in the detection of all Ser/Thr residues and largely hold $z \geq+3$ (Tables 2.1 \& 2.2).

\begin{tabular}{|c|c|l|c|c|c|}
\hline Mass & Position & \multicolumn{1}{|c|}{ Peptide } & \# HKR & \# ST & Comments \\
\hline 433.2 & $1-3$ & (DP)MKR & 2 & 0 & $?$ \\
\hline 2483.2 & $4-26$ & DHHQFQGRLSNHGTSSSSSS ISK & 5 & 9 & + \\
\hline 1267.6 & $27-36$ & DKMMMVKKEE & 3 & 0 & + \\
\hline 549.2 & $37-42$ & DGGGNM & 0 & 0 & $?$ \\
\hline 133.0 & $43-43$ & D & 0 & 0 & - \\
\hline 3953.0 & $44-78$ & $\begin{array}{l}\text { DELLAVLGYKVRSSEMAEVA } \\
\text { LKLELLTMMSNVQE }\end{array}$ & 3 & 4 & + \\
\hline 812.4 & $79-86$ & DGLSHLAT & 1 & 2 & + \\
\hline 1722.8 & $87-100$ & DTVHYNPSELYSWL & 1 & 3 & + \\
\hline 1965.0 & $101-119$ & DNMLSELNPPPLPASSNGL & 0 & 3 & + \\
\hline 1527.7 & $120-134$ & DPVLPSPEICGFPAS & 0 & 2 & + \\
\hline 296.1 & $135-136$ & DY & 0 & 0 & - \\
\hline 1758.0 & $137-152$ & DLKVIPGNAIYQFPAI & 3 & 8 & + \\
\hline 2024.9 & $153-171$ & DSSSSSNNQNKRLKSCSSP & 1 & 20 & $?$ \\
\hline 4003.0 & $172-212$ & DSMVTSTSTGTQIGGVIGTT & VTTTTTTTTAAGESTRSVIL V & & \\
\hline
\end{tabular}




\begin{tabular}{|c|c|l|c|c|c|}
\hline 7721.0 & $213-283$ & $\begin{array}{l}\text { DSQENGVRLVHALMACAEAI } \\
\text { QQNNLTLAEALVKQIGCLAV } \\
\text { SQAGAMRKVATYFAEALARR IYRLSPPQNQI }\end{array}$ & 8 & 5 & - \\
\hline 573.2 & $284-288$ & DHCLS & 1 & 1 & $?$ \\
\hline 4495.3 & $289-326$ & $\begin{array}{l}\text { DTLQMHFYETCPYLKFAHFT } \\
\text { ANQAILEAFEGKKRVHVI }\end{array}$ & 7 & 3 & + \\
\hline 3934.0 & $327-363$ & $\begin{array}{l}\text { DFSMNQGLQWPALMQALALR } \\
\text { EGGPPTFRLTGIGPPAP }\end{array}$ & 2 & 3 & $?$ \\
\hline 334.1 & $364-366$ & DNS & 0 & 1 & - \\
\hline 3565.8 & $367-398$ & DHLHEVGCKLAQLAEAIHVE FEYRGFVANSLA & 5 & 1 & + \\
\hline 246.1 & $399-400$ & DL & 0 & 0 & - \\
\hline 1117.5 & $401-410$ & DASMLELRPS & 1 & 2 & + \\
\hline 5750.2 & $411-463$ & $\begin{array}{l}\text { DTEAVAVNSVFELHKLLGRP } \\
\text { GGIEKVLGVVKQIKPVIFTV VEQESNHNGPVFL }\end{array}$ & 7 & 4 & $?$ \\
\hline 1777.8 & $464-477$ & DRFTESLHYYSTLF & 2 & 4 & + \\
\hline 1044.5 & $478-487$ & DSLEGVPNSQ & 0 & 1 & + \\
\hline 2395.2 & $488-509$ & DKVMSEVYLGKQICNLVACE GP & 2 & 1 & + \\
\hline 8579.2 & $510-587$ & $\begin{array}{l}\text { LAPAHLGSNAFKQASMLSV } \\
\text { FNGQGYRVEESNGCLMLGW } \\
\text { HTRPLITTSAWKLSTAAY }\end{array}$ & 15 & - \\
\hline
\end{tabular}

Table 2.1 - In silico AspN digestion of RGA. Residues belonging to the N-terminal 3xFLAG-tag are indicated in parentheses. Peptide masses (Da) and position numbers are listed along with the number of basic (\# HKR) and GIcNAc-modifiable (\# ST) residues for each theoretical peptide. The comments section refers to peptides predicted to be detected (+) or not detected (-), with (?) indicating uncertainty of detection.

\begin{tabular}{|c|c|l|c|c|c|}
\hline Mass & Position & Peptide & \# HKR & \# ST & Comments \\
\hline 277.1 & $1-2$ & (TDP)MK & 1 & 0 & - \\
\hline 174.1 & 3 & R & 1 & 0 & - \\
\hline 1023.5 & $4-11$ & DHHQFQGR & 3 & 0 & $?$ \\
\hline 1477.7 & $12-26$ & LSNHGTSSSSSSISK & 2 & 9 & + \\
\hline 261.1 & $27-28$ & DK & 1 & 0 & - \\
\hline 638.3 & $29-33$ & MMMVK & 1 & 0 & $?$ \\
\hline 146.1 & $34-34$ & K & 1 & 0 & - \\
\hline 2023.9 & $35-53$ & EEDGGGNMDDELLAVLGYK & 1 & 0 & + \\
\hline 273.2 & $54-55$ & VR & 1 & 0 & - \\
\hline 1063.5 & $56-65$ & SSEMAEVALK & 1 & 2 & + \\
\hline
\end{tabular}


Chapter 2

PTM Analysis of RGA

40

\begin{tabular}{|c|c|c|c|c|c|}
\hline 8140.8 & $66-139$ & $\begin{array}{l}\text { LEQLETMMSNVQEDGLSHLA } \\
\text { TDTVHYNPSELYSWLDNMLS } \\
\text { ELNPPPLPASSNGLDPVLPS PEICGFPASDYDLK }\end{array}$ & 3 & 12 & - \\
\hline 2550.2 & $140-163$ & VIPGNAIYQFPAIDSSSSSN NQNK & 1 & 5 & + \\
\hline 174.1 & $164-164$ & $\mathrm{R}$ & 1 & 0 & - \\
\hline 259.2 & $165-166$ & LK & 1 & 0 & - \\
\hline 3952.8 & $167-207$ & $\begin{array}{l}\text { SCSSPDSMVTSTSTGTQIGG } \\
\text { VIGTTVTTTTTTTTAAAGEST R }\end{array}$ & 1 & 22 & ? \\
\hline 1414.7 & $208-220$ & SVILVDSQENGVR & 1 & 2 & + \\
\hline 2663.4 & $221-245$ & LVHALMACAEAIQQNNLTLA EALVK & 2 & 1 & + \\
\hline 1403.7 & $246-259$ & QIGCLAVSQAGAMR & 1 & 1 & + \\
\hline 146.1 & $260-260$ & K & 1 & 0 & - \\
\hline 1210.6 & $261-271$ & VATYFAEALAR & 1 & 1 & + \\
\hline 174.1 & $272-272$ & $\mathrm{R}$ & 1 & 0 & - \\
\hline 450.3 & $273-275$ & IYR & 1 & 0 & - \\
\hline 3320.5 & $276-303$ & LSPPQNQIDHCLSDTLQMHF YETCPYLK & 3 & 4 & + \\
\hline 1892.9 & $304-320$ & FAHFTANQAILEAFEGK & 2 & 1 & + \\
\hline 146.1 & $321-321$ & K & 1 & 0 & - \\
\hline 174.1 & $322-322$ & $\mathrm{R}$ & 1 & 0 & - \\
\hline 2737.4 & $323-346$ & VHVIDFSMNQGLQWPALMQA LALR & 2 & 1 & + \\
\hline 859.4 & $347-354$ & EGGPPTFR & 1 & 1 & + \\
\hline 2156.0 & $355-375$ & LTGIGPPAPDNSDHLHEVGC K & 3 & 1 & + \\
\hline 1787.9 & $376-390$ & LAQLAEAIHVEFEYR & 2 & 0 & + \\
\hline 3744.9 & $391-425$ & $\begin{array}{l}\text { GFVANSLADLDASMLELRPS } \\
\text { DTEAVAVNSVFELHK }\end{array}$ & 3 & 5 & + \\
\hline 1038.6 & $426-435$ & LLGRPGGIEK & 2 & 0 & + \\
\hline 613.4 & $436-441$ & VLGVVK & 1 & 0 & ? \\
\hline 2765.5 & $442-465$ & QIKPVIFTVVEQESNHNGPV FLDR & 3 & 2 & + \\
\hline 2776.3 & $466-489$ & FTESLHYYSTLFDSLEGVPN SQDK & 2 & 6 & + \\
\hline 1024.5 & $490-498$ & VMSEVYLGK & 1 & 1 & + \\
\hline 1416.6 & $499-511$ & QICNLVACEGPDR & 1 & 0 & + \\
\hline 402.2 & $512-514$ & VER & 1 & 0 & - \\
\hline 1226.6 & $515-524$ & HETLSQWGNR & 2 & 2 & + \\
\hline 1659.8 & $525-541$ & FGSSGLAPAHLGSNAFK & 2 & 3 & + \\
\hline 1756.9 & $542-557$ & QASMLLSVFNSGQGYR & 1 & 3 & + \\
\hline
\end{tabular}




\begin{tabular}{|c|c|l|l|l|c|}
\hline 2728.3 & $558-581$ & VEESNGCLMLGWHTRPLITT SAWK & 3 & 5 & + \\
\hline 624.3 & $582-587$ & LSTAAY & 0 & 2 & $?$ \\
\hline
\end{tabular}

Table 2.2 - In silico trypsin digestion of RGA. Residues belonging to the N-terminal 3xFLAG-tag are indicated in parentheses. Peptide masses (Da) and position numbers are listed along with the number of basic (\# HKR) and GIcNAc-modifiable (\# ST) residues for each theoretical peptide. The comments section refers to peptides predicted to be detected (+) or not detected (-), with (?) indicating uncertainty of detection.

Each in silico digest alone produced a small population of peptides that were either too hydrophilic to bind to the $\mathrm{C} 18$ chromatographic column effectively or too hydrophobic to efficiently elute from the C18 during the reverse-phase gradient. However, the combined theoretical sequence coverage guaranteed by two distinct digestions using either AspN or trypsin was expected to potentially yield detection of all ST residues.

\subsubsection{Analysis of RGA by LCMS}

\section{LC-MS/MS Methodology}

Cysteine residues on whole RGA bound to anti-FLAG agarose beads were reduced with dithiothreitol and then irreversibly capped by alkylation using iodoacetamide. Following onbeads digestion, AspN- or tryptic-generated RGA peptides were loaded onto a C18 pre-column (PC), separated on a C18 analytical column (AC) by HPLC, and mass analyzed on an LTQ-FT-ICR or LTQ-Orbitrap mass spectrometer. Main beam or full $M S\left(\mathrm{MS}^{1}\right)$ scans of the intact peptides were acquired as they eluted according to their unique hydrophobicity along a $90 \mathrm{~min}$ reverse-phase chromatographic gradient. In this way, a powerful two-dimensional separation of the peptide mix was achieved; firstly by chromatographic retention and secondly by mass. In addition, attomole sensitivity could be achieved due to the use of micro-capillary reverse-phase chromatography columns, packed and conditioned in-house and equipped with micro-emitter tips to facilitate nanoflow micro-electrospray. The full MS scans of intact peptides were obtained by the FT-ICR (50,000 FWHM) or Orbitrap (60,000 FWHM) mass analyzers to take 
advantage of their superior mass resolution and accuracy, resolving $\mathrm{m} / \mathrm{z}$ differences of $<0.01 \mathrm{Da}$ at $400 \mathrm{~m} / z$ with mass accuracy errors of $<5$ parts per million (ppm).

Two internal standard peptides, Angiotensin (Angio) and Vasoactive Intestinal Peptide (Vaso) were spiked into every sample run at $100 \mathrm{fmol}$ prior to loading onto the PC. These internal standards were used to assess peptide loading, chromatographic quality, retention, mass accuracy, CAD and ETD fragmentation efficiency, and relative quantitation. Vaso and Angio were expected to maintain peak widths of 15-30 seconds ( $50 \%$ peak height) and observed retention times of $\sim 23$ and $\sim 32 \mathrm{~min}$, respectively, for a 90 min gradient. The experimental $\mathrm{MS}^{1}$ masses of intact peptide standard Vaso and Angio, with the theoretical monoisotopic masses of $[\mathrm{M}+3 \mathrm{H}]^{+3}: 475.8846 \mathrm{~m} / \mathrm{z}$ and $[\mathrm{M}+3 \mathrm{H}]^{+3}: 432.8998 \mathrm{~m} / \mathrm{z}$ respectively, were generally detected to be accurate to $<5 \mathrm{ppm}$, and the mass accuracy shifts of sample peptides were expected to agree with any mass accuracy shifts in the internal standards within this same $<5 \mathrm{ppm}$ window of error.

Mass resolution was calculated using Equation 2.1 where $W_{1 / 2}=$ the full width of the $\mathrm{m} / \mathrm{z}$ peak at half the maximum height (FWHM) and mass accuracy error was calculated in ppm using

\section{Equation 2.2.}

(Equation 2.1) mass resolution $=\frac{\left(\frac{m}{z}\right)}{\left(W_{\frac{1}{2}}\right)}$

By rearranging Equation 2.1 and solving for $W_{1 / 2}$, we find that a species with $400 \mathrm{~m} / z$ would be resolved from a separate species $\leq 399.992 \mathrm{~m} / \mathrm{z}$ and $\geq 400.008 \mathrm{~m} / \mathrm{z}$ using a mass resolution of 50,000 FWHM:

$$
\left(W_{\frac{1}{2}}\right)=\frac{(400)}{(50,000)}=0.008 \frac{\mathrm{m}}{\mathrm{z}}
$$


(Equation 2.2) ppm mass error $=\frac{\left(\left(\text { experimental } \frac{m}{z}\right)-\left(\text { theoretical } \frac{m}{z}\right)\right)}{\left(\text { theoretical } \frac{m}{z}\right)} \times 10^{6}$

By rearranging Equation 2.2 and solving for (experimental $\mathrm{m} / \mathrm{z}$ - theoretical $\mathrm{m} / \mathrm{z}$ ), we find that a species with $400 \mathrm{~m} / \mathrm{z}$ would be detected at $399.998-400.002 \mathrm{~m} / \mathrm{z}$ if the mass accuracy error is $<5$ ppm:

$$
\frac{(5)(400)}{10^{6}}=\left(\left(\text { experimental } \frac{m}{z}\right)-\left(\text { theoretical } \frac{m}{z}\right)\right)=0.002 \frac{\mathrm{m}}{z}
$$

Given these same resolution and mass accuracy parameters in the case of standard peptide Vaso, its $[\mathrm{M}+3 \mathrm{H}]^{+3}$ peak at $475.8846 \mathrm{~m} / \mathrm{z}$ could be resolved from neighboring species $\leq 475.8751$ $\mathrm{m} / \mathrm{z}$ and $\geq 475.8941 \mathrm{~m} / \mathrm{z}$, and would be detected between $475.8822-475.8870 \mathrm{~m} / \mathrm{z}$ :

$$
\begin{gathered}
\left(W_{\frac{1}{2}}\right)=\frac{(475.8846)}{(50,000)}=0.0095 \frac{\mathrm{m}}{\mathrm{z}} \\
\frac{(5)(475.8846)}{10^{6}}=\left(\left(\text { experimental } \frac{\mathrm{m}}{\mathrm{z}}\right)-\left(\text { theoretical } \frac{\mathrm{m}}{\mathrm{z}}\right)\right)=0.0024 \frac{\mathrm{m}}{\mathrm{z}}
\end{gathered}
$$

The ability to resolve fine differences in mass with mDa mass accuracy dramatically increases the ability to confidently identify RGA peptides and post-translational modifications, even when species very close in mass are present. One invaluable aspect of using such high resolution and mass accuracy is that it accesses the ability to distinguish between atomic isotopes. These isotopes differ by the mass of one or more neutrons (1.0087 Da/neutron) minus a small mass defect caused by the amount of nuclear binding energy needed for the neutron to form part of the nucleus of the element ${ }^{50}$. As these mass defects differ for each isotope of 
every element, Carbon-12 has been defined as the element having no mass defect and a

monoisotopic mass of $12.0000 \mathrm{Da}$. The two elements present in all proteins that most contribute to multiple peptidic isotopes are carbon and nitrogen. Carbon's most abundant natural isotope, carbon-12, is present at $98.9 \%$ abundance while $1.1 \%$ is present as carbon-13, with a mass of $13.0034 \mathrm{Da}$. The most abundant natural isotope of nitrogen, nitrogen-14 with a mass of 14.0031 , is present at $99.6 \%$, with its lesser abundant isotope, nitrogen-15 at mass 15.0001 , present at $0.37 \%$. On average, peptides contain five carbons per residue but only one nitrogen. Carbon-13 has a greater effect than nitrogen-15 on the isotopic distribution of peptides, because it is a more abundant isotope and because of the disparity between the number of carbon and nitrogen atoms. For this reason, reasonable first-order approximations of a peptide's isotopic distribution may be made by only considering carbon- 13 isotopes. The probability of a monoisotopic peptide, having only carbon-12 atoms $\left(\mathrm{P}_{\mathrm{M}}\right)$, is given by Equation 2.3, where $c$ is the abundance of carbon- 13 and $w$ is the number of total carbon items in the peptide ${ }^{51}$ :

(Equation 2.3) $P_{M}=\left(\frac{100-c}{100}\right)^{w}$

Likewise, the probability of a peptide containing one carbon-13 atom $\left(\mathrm{P}_{\mathrm{M}+1}\right)$ is given by Equation 2.4:

(Equation 2.4) $P_{M+1}=w\left(\frac{c}{100-c}\right)\left(\frac{100-c}{100}\right)^{w}$

For Vaso, bearing 61 carbons, the probability that one of its molecules contains only carbon-12 is $51 \%$ :

$$
P_{M}=\left(\frac{100-1.1}{100}\right)^{61}=\left(\frac{98.9}{100}\right)^{61}=51 \%
$$


The probability that Vaso contains exactly one carbon-13 atom is therefore $34 \%$ :

$$
P_{M+1}=61\left(\frac{1.1}{100-1.1}\right)\left(\frac{100-1.1}{100}\right)^{61}=(61)(0.011)(.989)^{61}=34 \%
$$

Alternatively, the probability of isotopic peaks caused by carbon-13 may be determined by binomial probability ${ }^{52}$. The probability $P_{k}$ of a peptide containing exactly $k$ carbon-13 atoms with $\mathrm{n}$ total carbons atoms may be calculated from Equation 2.5:

$$
\text { (Equation 2.5) } P_{k}=\left(\begin{array}{l}
n \\
k
\end{array}\right)\left(p^{k}\right)(1-p)^{n-k}
$$

Therefore, the probabilities of Vaso containing exactly zero, one, and two carbon-13 atoms are calculated as $51 \%, 34 \%$, and $12 \%$ respectively:

$$
\begin{gathered}
P_{k}=\left(0.011^{0}\right)(1-0.011)^{61-0}=51 \% \\
P_{k}=\frac{61 !}{1 !(61-1) !}\left(0.011^{1}\right)(1-0.011)^{61-1}=34 \% \\
P_{k}=\frac{61 !}{2 !(61-2) !}\left(0.011^{2}\right)(1-0.011)^{61-2}=12 \%
\end{gathered}
$$

These probabilities translate to the amount of total Vaso ion current that will be represented in each isotopic state. One half of the ions will represent the first isotopic peak pertaining to carbon 12 only, a third will represent the second peak, and the remaining third of the ion current will contain the remaining peaks, which contain two or more carbon-13 atoms (i.e. $2{ }^{13} \mathrm{C}$, $3^{13} \mathrm{C}$, etc.). The relative abundances of each carbon-13 peak vs. carbon- 12 only are easily determined by using the products of Equation $\mathbf{2 . 5}$ to solve Equation $\mathbf{2 . 6}$ where the peptide contains k carbon-13 atoms:

(Equation 2.6) Relative abundance of $k^{13} C=\frac{P_{k}}{P_{0}}$ 
Using probabilities $\mathrm{P}_{0}, \mathrm{P}_{1}, \mathrm{P}_{2}$, find that ions containing one and two carbon-13 atoms are present at $67 \%$ and $24 \%$ respectively, relative to those containing only carbon-12:

$$
\begin{aligned}
& \text { Relative abundance of } 1^{13} \mathrm{C}=\frac{34 \%}{51 \%}=67 \% \\
& \text { Relative abundance of } 2{ }^{13} \mathrm{C}=\frac{12 \%}{51 \%}=24 \%
\end{aligned}
$$

When the identity of a peptide is unknown, an estimation of the number of residues, and therefore the number of carbons may be made. The average molecular weight of all 20 amino acids is $110 \mathrm{Da}$, so an approximation of the total number of carbons can be made using Equation

2.7:

(Equation 2.7) \# Carbon atoms $=\left(\frac{\text { Total Molecular weight Da }}{110 D a}\right)(5)$

Applying this calculation to Vaso yields

$$
\# \text { Carbon atoms }=\left(\frac{1425}{110}\right)(5)=65
$$




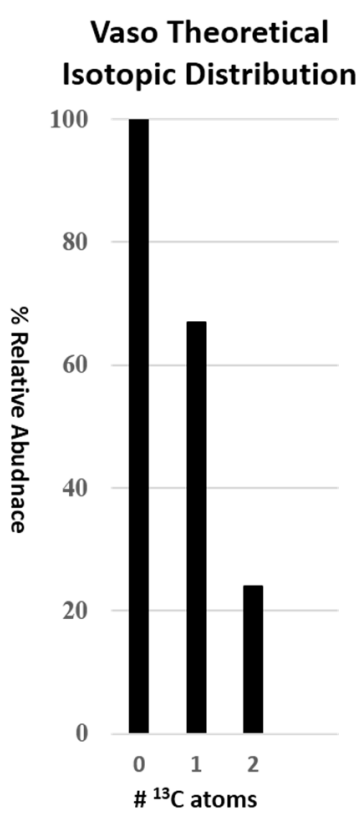

Figure 2.14 - Theoretical distribution of the first three isotopic peaks of Vasoactive Intestinal Peptide fragment, HSDAVFTDNYTR. The distribution was computed binomially using equations 2.5 and 2.6. which is close to the actual number of 61 carbons. The theoretical isotopic distribution of $[\mathrm{M}+3 \mathrm{H}]^{+3}$ Vaso, computed binomially from Equations 2.5 and 2.6, is shown in (Figure 2.14).

By comparison, Vaso's experimental isotopic distribution (Figure 2.15) matches well with the Vaso Experimental Isotopic Distribution

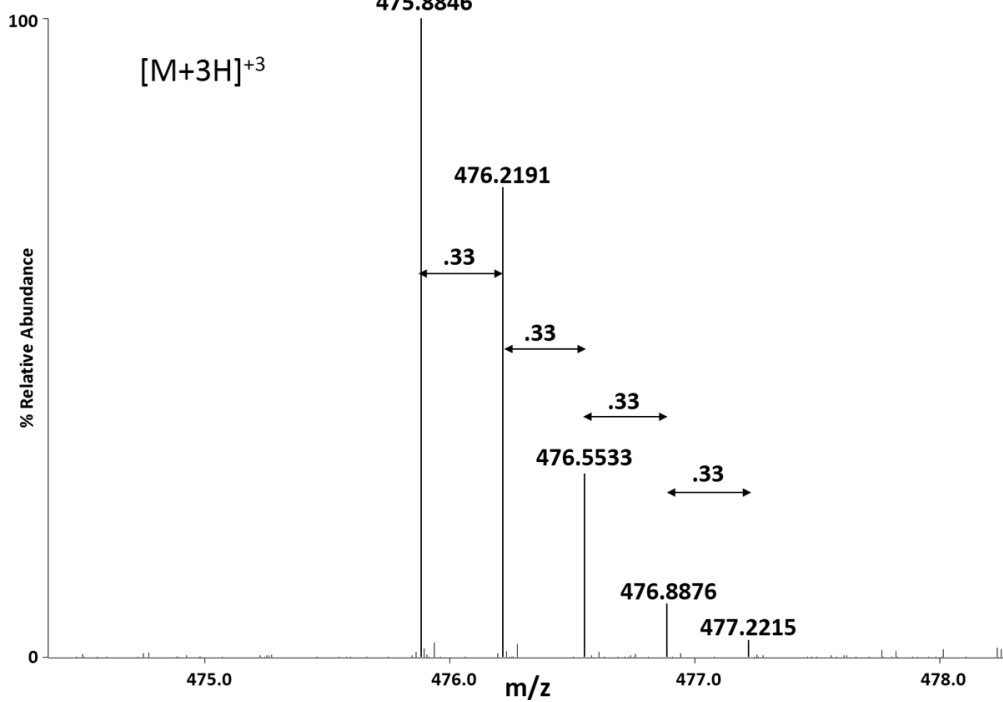

Figure 2.15 - Isotopic distribution of Vasoactive Intestinal Peptide fragment, HSDAVFTDNYTR, experimentally determined using LTQ-FT at 50,000 FWHM resolution.

statistical approximation. It is important to note that the isotopic distribution of Vaso and all 
peptides will be completely independent of charge state because the only difference in primary molecular structure is the degree of protonation. However, the ability to resolve isotopes has a critical implication for determining charge states by mass spectrometry. Because each successive isotopic peak represents a molecule separated by $\sim 1 \mathrm{Da}$ and the mass spectrometer measures $\mathrm{m} / \mathrm{z}$, the pattern of separation between isotopic peaks within a molecule is indicative of its charge state. One may simply solve for charge state by Equation $\mathbf{2 . 8}$ when the difference in $\mathrm{m} / \mathrm{z}$ between isotopic peaks ( $\Delta$ isotopes) is known:

$$
\text { (Equation 2.8) } z=\left(\frac{1}{\Delta \frac{m}{z} \text { between isotopes }}\right)
$$

For the most abundant charge state of Vaso $(z=3)$, the distance between isotopes is $0.33 \mathrm{~m} / \mathrm{z}$ so this equates to:

$$
\text { Vaso } z=\left(\frac{1}{0.33}\right)=3
$$

Due to the high resolving power of mass analyzers like the FT-ICR and Orbitrap, the charge states of peptides from RGA samples could be determined, allowing not only a highly accurate $\mathrm{m} / \mathrm{z}$ measurement, but that of mass itself. In addition, isotopic distributions were used as an analytical tool, giving an additional piece of identifying evidence for alleged peptides. Likewise, analysis of isotopic distribution helped determine if species similar in mass were potentially related as these distributions should be virtually identical barring the addition of atoms with highly abundant isotopes, uncommon to most peptides, or low abundance/poor detection of ions that skews the statistics of isotopes detected. These observations were made both qualitatively and quantitatively with the aid of the PNNL Isotope Pattern Calculator, created by Michael Cusack. It is important to note that one distinct disadvantage encountered in resolving isotopes is that a peptide's ion current, detected as one distinct peak under low resolution, is 
split amongst multiple signals in high-resolution, lowering the signal-to-noise ratio by a certain factor. As previously calculated, $51 \%$ of Vaso's ion current is due to its ${ }^{12} \mathrm{C}$ peak. The abundance of the ${ }^{12} \mathrm{C}$ peak will decrease as peptide mass increases and the overall number of isotopic peaks detected will increase as they become more abundant. This, combined with the tendency for peptides to also gain a larger charge state distribution as they increase in mass, can drastically divide ion current. This is one of the greatest analytical challenges associated with analyzing large peptides and whole proteins, techniques termed middle- and top-down, respectively.

RGA peptides were quantified relative to the levels of standard peptides Angio and Vaso detected. Approximately 100 fmols of each peptide standard were spiked into every sample before they were loaded onto the pre-column. The monoisotopic peaks corresponding to each charge state of each standard were manually observed by isolating them in an extracted ion chromatogram (XIC) with a $0.01 \mathrm{~m} / \mathrm{z}$ window centered on the theoretical $\mathrm{m} / \mathrm{z}$ value). Figure 2.16 demonstrates standards from a representative run of trypsin digested RGA. The ion current corresponding to $100 \mathrm{fmol}$ was calculated by integrating the total C12 isotope area of each standard and averaging the two using Equation 2.9:

(Equation 2.9) $100 \mathrm{fmol}$ ion current $=\left(\frac{\sum \text { Area }_{100} \mathrm{fmol} \mathrm{Angio}_{1}+\sum \text { Area }_{100 \mathrm{fmol} \mathrm{Vaso}}}{2}\right)$ Using the same methodology and the data from Equation 2.9, Equation 2.10 was used to calculate the abundance of RGA peptides (including all PTM forms) and other species in a single analysis:

$$
\text { (Equation 2.10) Peptide abundance }(\mathrm{fmol})=\frac{100\left(\sum \text { Area }_{\text {sample peptide })}\right.}{(100 \text { fmol ion current })^{-1}}
$$

For the tryptic RGA peptide run shown in Figure 2.16, $100 \mathrm{fmol}$ equated to: 


$$
100 \text { fmol ion current }=\left(\frac{6.09 E 8+6.72 E 8}{2}\right)=6.41 \mathrm{E} 8
$$

and the abundance of the unmodified tryptic RGA peptide, FGSSGLAPAHLGSNAFK, was calculated to be:

$$
\text { Peptide abundance }(\mathrm{fmol})=\frac{100(4.75 E 9)}{(6.41 E 8)}=741 \mathrm{fmol}
$$

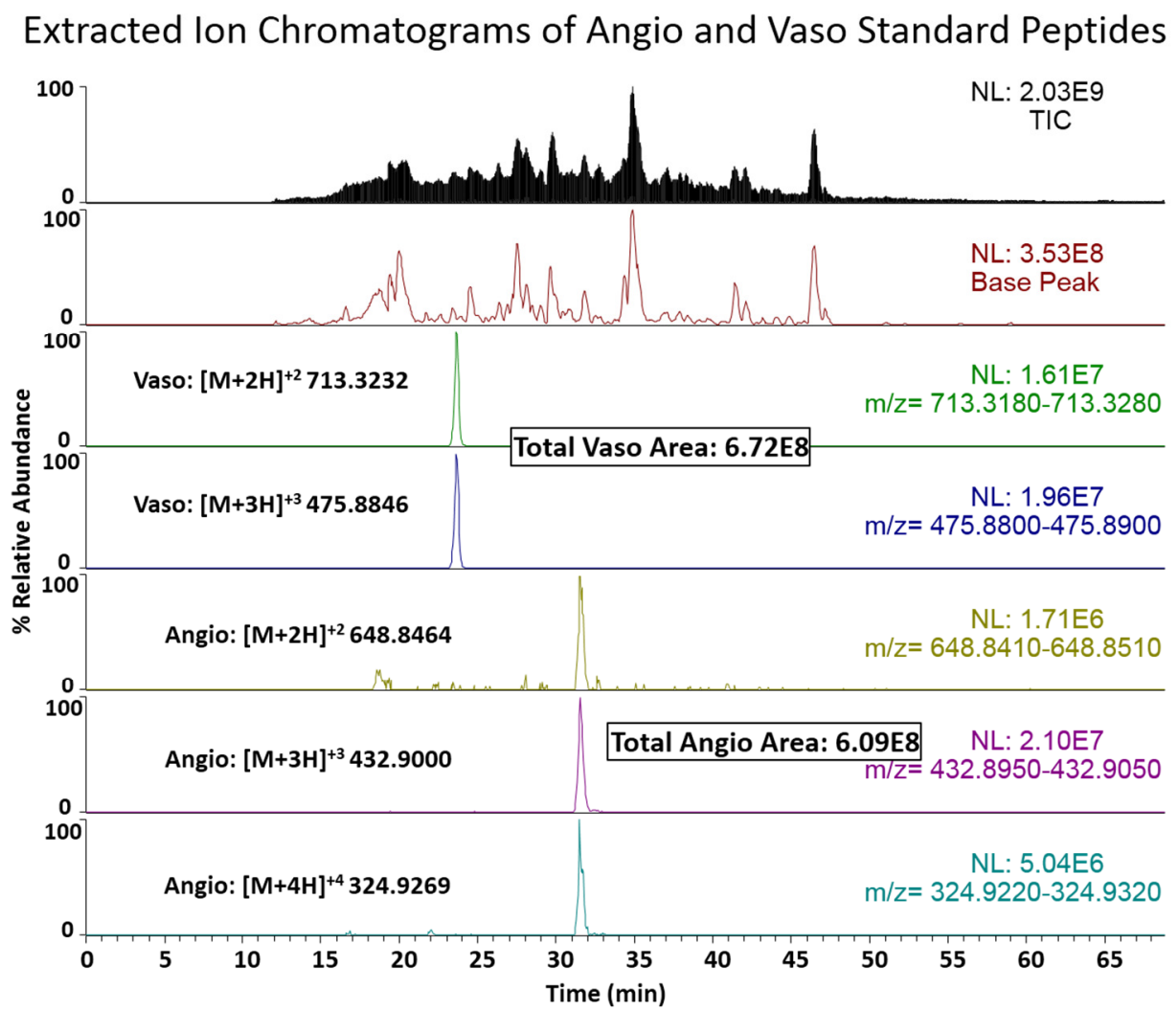

Figure 2.16 - XICs of internal peptide standards Vasoactive Intestinal Peptide (Vaso) and Angiotensin (Angio) used to calculate relative quantitation of RGA peptides.

While variations in isotopic distribution between peptides exist due to different sizes and sequences, the effect on abundance calculations was negligible considering the population of RGA peptides analyzed. Most peptides ranged from 10-25 residues in length, with the percentage of monoisotopic peaks in the overall isotopic distribution differing by a factor $\leq 2$. Therefore, comparison of the monoisotopic abundances of known amounts of standards relative 
to those of sample peptides offered a robust method to calculate sample peptide abundances.

Still, variations in the levels of peptides detected varied as is expected due to factors such as digestion efficiency and homogeneity, complete cysteine reduction and alkylation, peptide solubility, binding and elution efficiency on C18, variable sample complexity throughout chromatographic gradient, and ionization efficiency by ESI. In order to estimate the approximate amount of RGA present in each digest, the abundances of the top five most abundant RGA peptides were summed and averaged (Equation 2.11):

$$
R G A \text { abundance }(f m o l)=\bar{x}_{\text {top } 5} \text { abundant } R G A \text { peptides }
$$

In order to control for the variability in peptide levels detected, the percent total abundance of post-translationally modified peptides was quantified relative to the total abundance of all forms of those peptides detected (Equation 2.12), rather than against the estimated protein abundance as a whole.

(Equation 2.12) \% Abundance Modified Peptide $=\frac{\sum \text { Area }_{\text {Modified peptide }}}{\sum \text { Area }_{\text {All peptide forms }}} \times 100$ Detection of low-level PTMs

High sample purity, intelligent instrumentation methods, and care interpreting data are all critical for the successful detection of O-GlcNAcylated peptides, as they are usually detected at low levels $(<10 \%)$ relative to their unmodified counterparts. The dynamic range $(>1000)$ and sensitivity (attomole detection) of the high-resolution trap-based mass analyzers utilized allows for the detection of PTMs at $\leq 1 \%$, but this sensitivity will be lost if a significant amount of other ions, including unmodified forms of the desired peptide to be analyzed, fill the trap's capacity. This prevents one from simply analyzing a larger amount of material to improve O-GIcNAc detection. For $\mathrm{MS}^{2}$ analysis, the LTQ is filled exclusively with ions that display the specific $\mathrm{m} / \mathrm{z}$ 
value to be fragmented, bypassing this issue unless an abundant contaminating ion has a massto-charge ratio within the isolation window (typically $3 \mathrm{~m} / \mathrm{z}$ in total). Still, low PTM abundance presents a challenge for obtaining quality fragmentation and $\mathrm{MS}^{2}$ spectra for two reasons.

Firstly, the mass spectrometer is generally operated in the data-dependent mode whereby ions detected in $\mathrm{MS}^{1}$ are automatically chosen for $\mathrm{MS}^{2}$ analysis based on parameters described in Methods to obtain $\mathrm{MS}^{2}$ data for as many analytes as possible. Low level species like most OGlcNAcylated peptides may still be too low to be chosen over higher abundant species coeluting with them. For individual cases where the $\mathrm{m} / \mathrm{z}$ value of a particular PTM peptide is known, this may be circumvented by operating in data-independent mode in which ions that fall within the targeted $\mathrm{m} / \mathrm{z}$ window will continually undergo $\mathrm{MS}^{2}$ regardless of its abundance in the preceding $\mathrm{MS}^{1}$ spectra. Secondly, O-GIcNAc's low abundance is often not sufficient to fill the ion trap to its optimal capacity for conducting $\mathrm{MS}^{2}$, resulting in poor spectral quality that prevents confident sequencing and PTM site-localization. In addition to these challenges, some OGlcNAcylated peptides appear to suffer from "ion suppression" whereby the O-GlcNAcylated peptide, which has only a slightly lower retention by reverse-phase chromatography than its unmodified counterpart, fails to ionize as efficiently (Figure 2.17). 


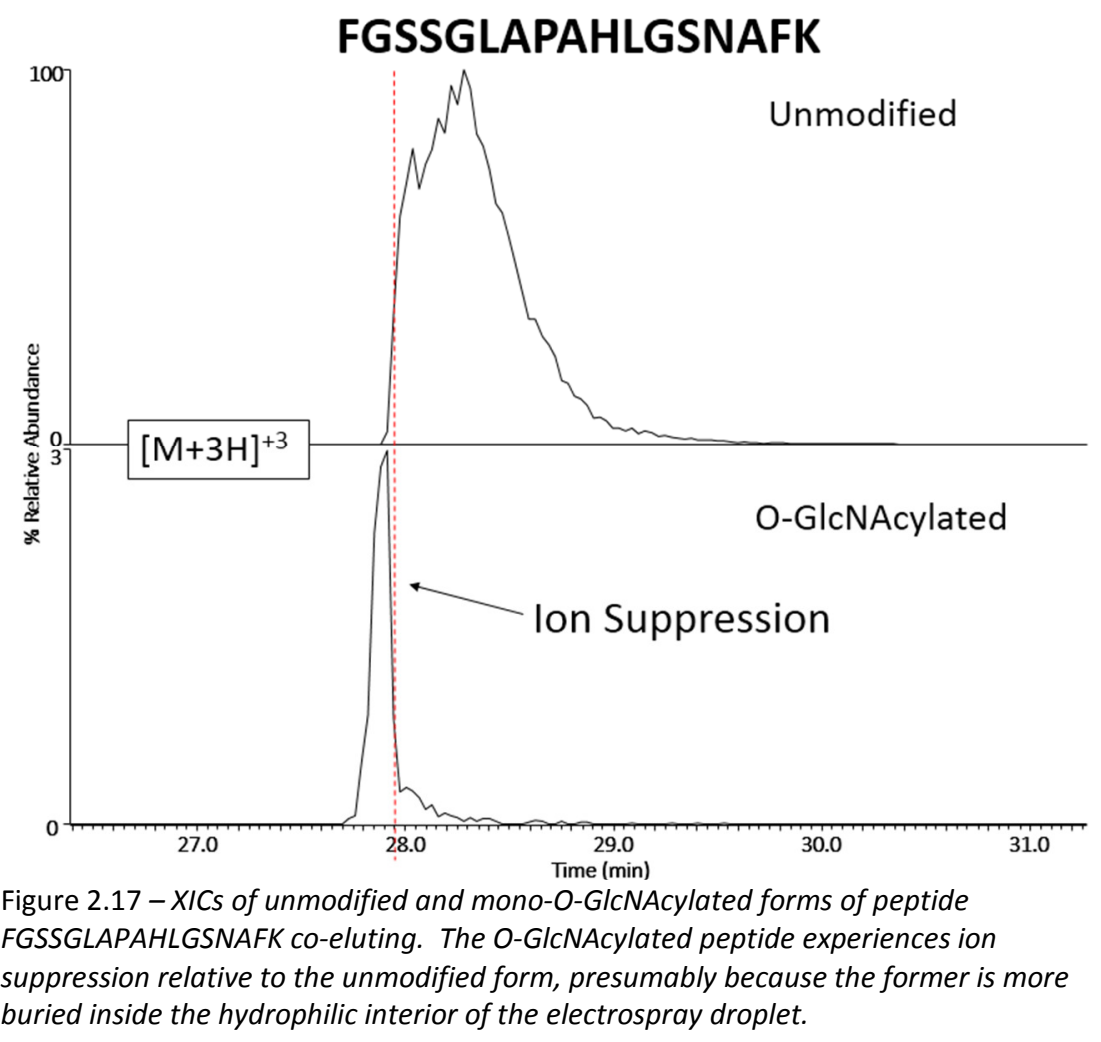

One hypothesis for this phenomenon fits well with the ion desorption model of electrospray ionization ${ }^{53}$ postulating that analyte ions are created when the surface electric fields of the charged electrospray droplets become great enough to allow an analyte ion to escape the energy barrier holding it inside the droplet. Under this model, it is conceivable that O-GIcNAc ion suppression could occur, because the slightly more hydrophilic O-GlcNAcylated peptides may favor the aqueous interior of the ESI droplet over the more hydrophobic liquid-gas interphase, where desorption and ionization occurs. Researchers have developed various OGIcNAc enrichment methods to overcome these limitations. These include immunoprecipitation by the pan-specific O-GlcNAc antibody, CTD110.6, raised against the carboxyl terminal domain of O-GlcNAcylated RNA polymerase $\|^{54,55}$, lectin weak affinity chromatography ${ }^{56}$, and chemoenzymatic tagging to enrich O-GIcNAc using biotin-avidin affinity ${ }^{31,54,55}$. While all of these methods have shown some success using large amounts of whole cell or nuclear lysates, 
none have demonstrated the ability to enrich O-GIcNAcylation with high recovery or reproducibility. While efforts are continually being made to development an enrichment method that will efficiently eliminate non-GlcNAcylated material while recovering OGlcNAcylated peptides in high yield, none yet exists that is appropriate for characterizing GIcNAcylation across a whole protein like RGA. To maximize the probability of successfully detecting O-GIcNAcylation, we relied on the combination of SEC overexpression, immunoprecipitation efficiency, appropriate sample preparation, sensitive LCMS instrumentation, intelligent MS methodology, and thoughtful, knowledgeable data analysis.

\section{Sequencing Peptides using MS/MS analysis}

Because ESI is a soft ionization technique, ionized peptides are left intact and may be analyzed accordingly to generate the numerous data already discussed. Still, the absence of fragmentation yields little information to investigate the primary structure and sequence of these peptides. MS/MS analysis enabled RGA peptide sequencing by fragmenting the peptide ions to generate product fragment ions, and mass analyzing those ions to generate sequence ladder data. The resulting spectra were interpreted to determine peptide primary structure and locate PTM sites. CAD and ETD MS/MS were both utilized and conducted in the LTQ in order to take advantage of its speed and sensitivity. Approximately 3-5 MS/MS spectra at $1 \mathrm{~m} / \mathrm{z}$ unit resolution (400 FWHM) and $\pm 0.2 \mathrm{~m} / \mathrm{z}$ mass accuracy could be acquired at $\sim 10$-fold higher sensitivity in the same time frame ( $1 \mathrm{sec})$ as $1 \mathrm{MS}^{1}$ scan in the FT-ICR or Orbitrap. Open Mass Spectrometry Search Algorithm (OMSSA) software aided in data analysis to search for OGlcNAcylation and phosphorylation PTMs, as well as non-specifically immunopurified tobacco proteins. Still, theoretical monoisotopic masses for all potentially modified RGA peptides were 
manually investigated and all RGA MS/MS spectra manually validated for peptide sequencing and PTM site-mapping.

MS/MS Sequencing by CAD

Fragmenting peptides by CAD has enabled mass spectrometrists to unambiguously identify peptide sequences, and locate certain PTM sites. However, the presence of labile Olinked PTMs like O-GIcNAc and phosphorylation on a peptide severely decrease the amount of random peptide bond cleavages, therefore suppressing the number and abundance of $\mathrm{b}$ and $\mathrm{y}$ ions available to facilitate sequencing. This is due to the presence of significantly more labile Olinkages (relative to peptide bonds) that upon CAD, preferentially result in the neutral loss of phosphoric acid (phosphorylation) or charge-reduced loss of an oxonium ion (O-GlcNAcylation)

${ }^{54}$ from modified peptides. This phenomenon can be exploited as an analytical tool, enabling one to search for these diagnostic ions to identify modified peptides. While phosphorylation can sometimes be site-mapped using CAD due to incomplete fragmentation of the labile Ser/Thrphosphate linkage, it is typically impossible to determine sites of O-GIcNAcylation by CAD fragmentation. O-GlcNAcylated peptides commonly fragment completely and exclusively at the Ser/Thr-O-GIcNAc linkage and prevent any amide bond fragmentation. The difficulty in site localization of O-GIcNAcylated peptides is compounded by the fact that O-GIcNAcylation often exists in Ser/Thr-rich domains of proteins ${ }^{7}$, causing further ambiguity in locating the modified site as multiple putative locations exist in high density. RGA contains four such regions (Figure 2.18), and it is imperative to generate a nearly perfect series of fragment ions to identify PTMs in them. 
Site-mapping O-GIcNAcylation by ETD

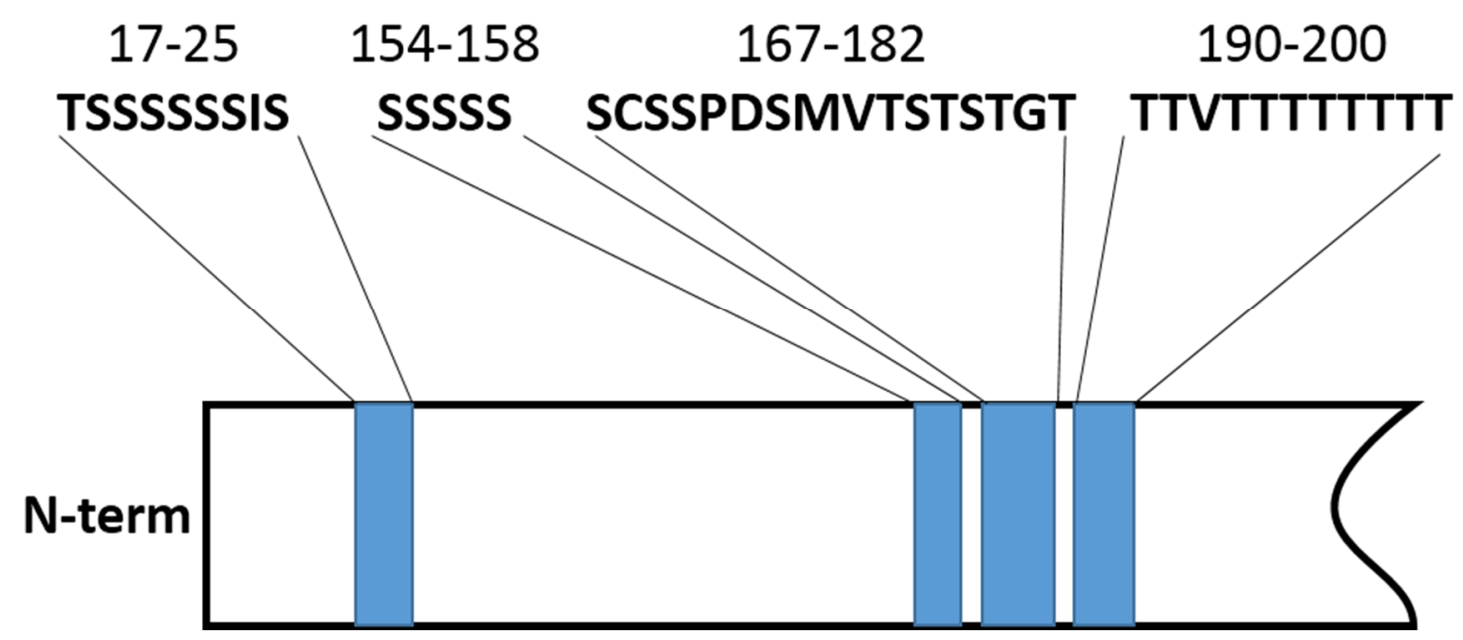

Figure 2.18 -Schematic of 4 Ser/Thr-rich domains in RGA. All 4 are located in the N-terminal half of RGA.

Fortunately, ETD offers an alternative MS/MS method with the ability to fragment

peptides along the backbone and retain O-GIcNAcylation on the modified residue by utilizing a fragmentation pathway based on free-radical chemistry rather than collisions with helium atoms. The O-GIcNAc site(s) can be localized using ETD while CAD produces only diagnostic fragment ions (Figure 2.19). 

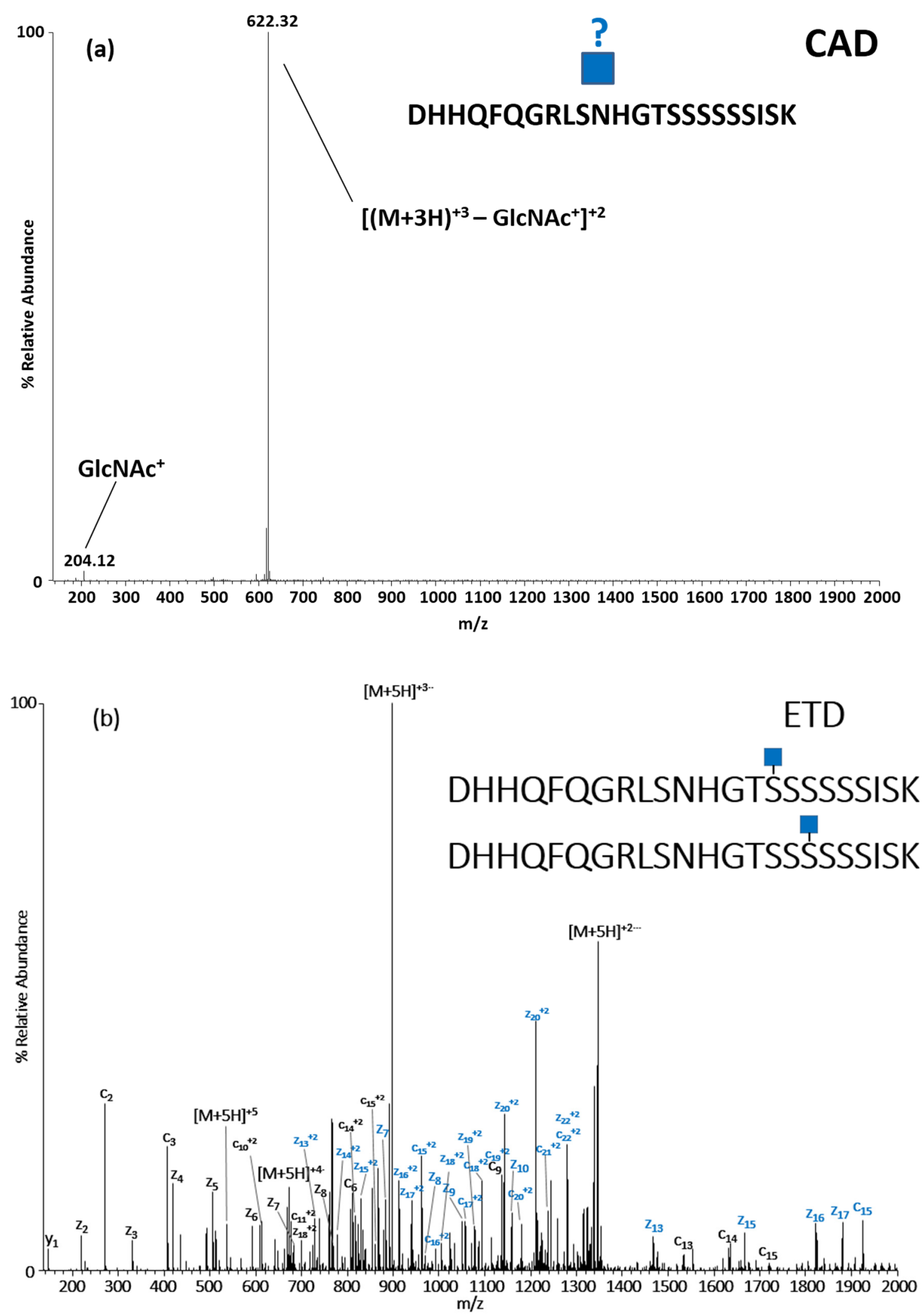
(c)

\begin{tabular}{|c|c|c|c|c|c|c|c|c|c|c|c|c|}
\hline \multicolumn{2}{|c|}{ GlcNAC } & \multicolumn{2}{|c|}{ Unmodified } & \multirow[b]{2}{*}{ C } & & \multirow[b]{2}{*}{ Z } & \multicolumn{3}{|c|}{ Unmodified } & \multicolumn{3}{|c|}{ GlcNAC } \\
\hline $\begin{array}{l}+2 \mathrm{c} \\
\text { ion }\end{array}$ & $\begin{array}{l}+1 \mathrm{c} \\
\text { ion }\end{array}$ & $\begin{array}{l}+2 \mathrm{c} \\
\text { ion }\end{array}$ & $\begin{array}{l}+1 \mathrm{c} \\
\text { ion }\end{array}$ & & & & $\begin{array}{l}+1 \mathrm{z} \\
\text { ion }\end{array}$ & $\begin{array}{l}+2 z \\
\text { ion }\end{array}$ & $\begin{array}{l}+3 z \\
\text { ion }\end{array}$ & $\begin{array}{l}+1 \mathrm{z} \\
\text { ion }\end{array}$ & $\begin{array}{l}+2 \mathrm{z} \\
\text { ion }\end{array}$ & $\begin{array}{l}+3 z \\
\text { ion }\end{array}$ \\
\hline & & 67 & 133 & 1 & D & 23 & 2484 & 1243 & 829 & 2687 & 1345 & 897 \\
\hline & & 136 & 270 & 2 & H & 22 & 2353 & 1178 & 786 & 2556 & 1279 & 853 \\
\hline & & 204 & 407 & 3 & H & 21 & 2216 & 1109 & 740 & 2419 & 1211 & 808 \\
\hline & & 268 & 535 & 4 & $\mathrm{Q}$ & 20 & 2079 & 1041 & 694 & 2282 & 1142 & 762 \\
\hline & & 342 & 682 & 5 & $\mathrm{~F}$ & 19 & 1951 & 977 & 651 & 2154 & 1078 & 719 \\
\hline & & 406 & 810 & 6 & Q & 18 & 1804 & 903 & 602 & 2007 & 1005 & 670 \\
\hline & & 434 & 867 & 7 & G & 17 & 1676 & 839 & 560 & 1879 & 940 & 627 \\
\hline & & 513 & 1023 & 8 & $\mathbf{R}$ & 16 & 1619 & 810 & 541 & 1822 & 912 & 608 \\
\hline & & 569 & 1137 & 9 & L & 15 & 1463 & 732 & 489 & 1666 & 834 & 556 \\
\hline 714 & 1427 & 613 & 1224 & 10 & S & 14 & 1350 & 676 & 451 & 1553 & 777 & 519 \\
\hline 771 & 1541 & 670 & 1338 & 11 & $\mathrm{~N}$ & 13 & 1263 & 632 & 422 & 1466 & 734 & 490 \\
\hline 840 & 1678 & 738 & 1475 & 12 & H & 12 & 1149 & 575 & 384 & 1352 & 677 & 451 \\
\hline 868 & 1735 & 767 & 1532 & 13 & G & 11 & 1011 & 507 & 338 & 1215 & 608 & 406 \\
\hline 919 & 1836 & 817 & 1633 & 14 & $T$ & 10 & 954 & 478 & 319 & 1158 & 580 & 387 \\
\hline 963 & 1923 & 861 & 1720 & 15 & $\mathrm{~s}$ & 9 & 853 & 427 & 285 & 1056 & 529 & 353 \\
\hline 1006 & 2010 & 904 & 1807 & 16 & $S$ & 8 & 766 & 384 & 256 & 969 & 486 & 324 \\
\hline 1050 & 2097 & 948 & 1894 & 17 & S & 7 & 679 & 340 & 227 & 882 & 442 & 295 \\
\hline 1093 & 2184 & 992 & 1981 & 18 & S & 6 & 592 & 297 & 198 & 795 & 398 & 266 \\
\hline 1137 & 2271 & 1035 & 2068 & 19 & $S$ & 5 & 505 & 253 & 169 & 708 & 355 & 237 \\
\hline 1180 & 2358 & 1079 & 2155 & 20 & $S$ & 4 & 418 & 210 & 140 & 621 & 311 & 208 \\
\hline 1237 & 2471 & 1135 & 2268 & 21 & 1 & 3 & 331 & 166 & 111 & 534 & 268 & 179 \\
\hline 1280 & 2558 & 1179 & 2355 & 22 & $S$ & 2 & 218 & 110 & 73 & 421 & 211 & 141 \\
\hline 1345 & 2687 & 1243 & 2484 & 23 & K & 1 & 131 & 66 & 44 & & & \\
\hline
\end{tabular}

Figure 2.19-CAD (a) and ETD (b) spectra of mono-O-GlcNAcylated peptide DHHQFQGRLSNHGTSSSSSSISK. Signature fragmentation of the GICNAC moiety indicates the peptide is O-GICNAcylated, but no sequence data was generated. The $c$ and $z$. fragment ions produced by ETD $(b \& c)$ indicated that two mono-O-GlcNAcylated forms co-eluted and were fragmented together. One form contained O-GICNAC on S18 and another on S2O, highlighted in blue in (c). Unmodified fragment ions are indicated in gray/black and O-GlcNAcylated in blue (b \& c). Blue square indicates site of O-GlcNAcylation.

Another advantage of ETD is that it can effectively fragment peptides of larger size if they contain sufficient charge, whereas CAD fragmentation becomes less efficient as peptide length increases and vibrational energy diffuses across a larger mass. The ETD fragmentation mechanism requires that the carbonyl radical anion (generated by electron transfer from radical anion reagent) abstract a proton from a nearby protonated nitrogen, causing the radical site located on the resulting charge-reduced species to trigger fragmentation of the $\mathrm{N}-\mathrm{C} \alpha$ bond resulting in ions of type $c$ and $z \cdot{ }^{57}$. Because of this, the most critical requirements for candidate peptides are that they contain $z \geq+3$ with the charge ideally spread evenly across the peptide.

This allows one charge to remain on each $\mathrm{c}$ and $z$ : ion after charge-reduction, and ensures that protons will be available to facilitate fragmentation along the entire peptide backbone. Increasing the charge also has the added benefit of increasing the ETD reaction rate as the rate 
of electron transfer from the reagent radical anion to the cationic peptide increases

quadratically with the peptide precursor charge state ${ }^{58}$ (e.g. a peptide of $z=+30$ will react $100 x$ faster than that of $z=+3$ ). Therefore, ETD is not only superior to CAD for purposes of sitemapping labile PTMs like O-GIcNAcylation, it is also superior in that it can be used on longer (>15 residues), more highly-charged ( $z \geq+3)$ peptides, giving a greater understanding of what PTMs exist simultaneously or individually on the protein as a whole.

\subsubsection{His-Flag-RGA (+SEC) Sequence Coverage}

Figures $2.20 \mathrm{~A} \& 2.20 \mathrm{~B}$ respectively demonstrate representative base peak chromatograms of AspN and tryptic RGA digest screens, reporting the most abundant ion detected in each MS1 scan. Screens of 500-1000 fmol RGA were extrapolated to determine that 6xHis-3xFLAG RGA(+SEC) samples each contained 10-50 pmols of RGA. 

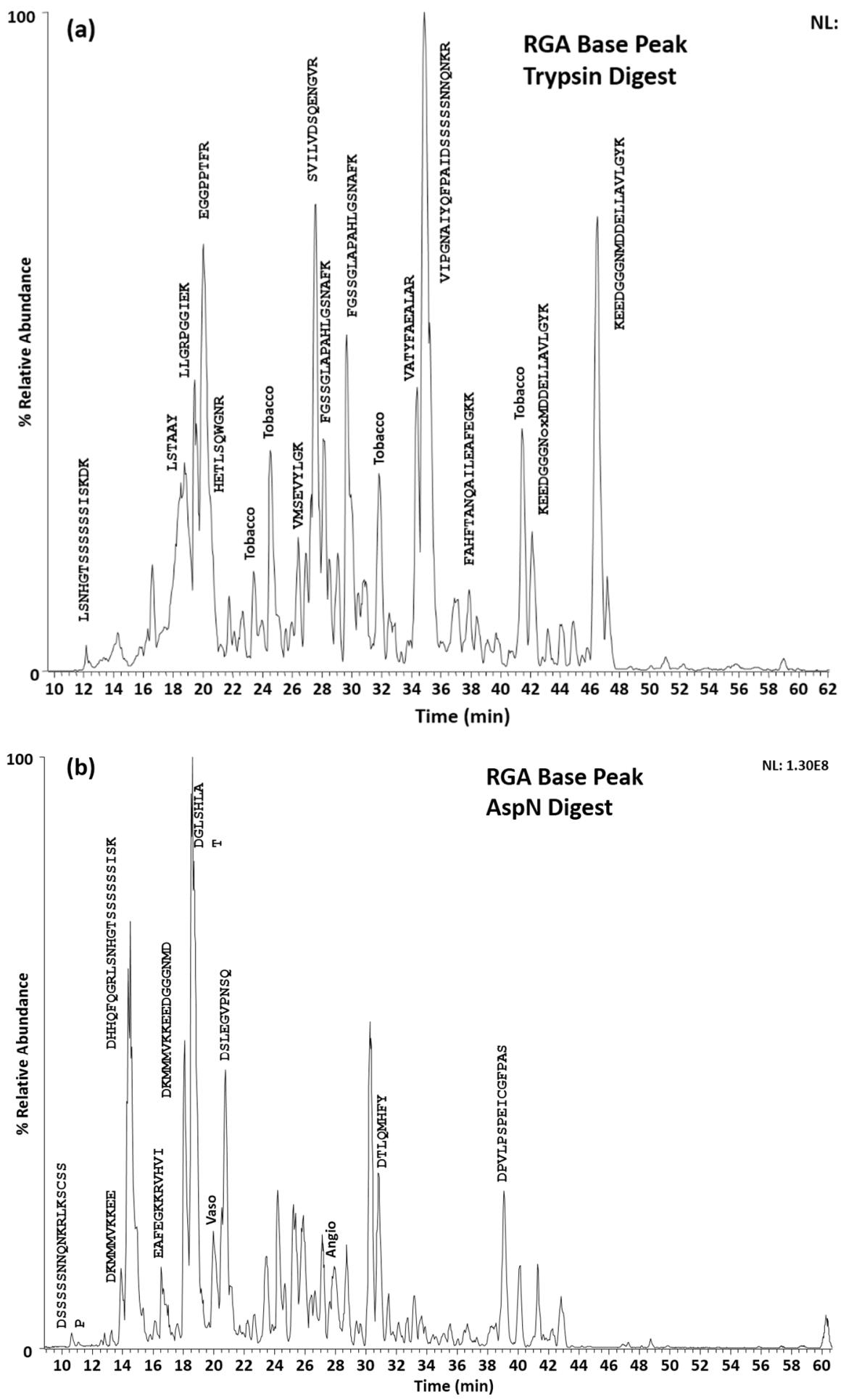

Figure 2.20-Base Peak Chromatograms of representative trypsin (a) and AspN (b) RGA digests. 
Notably, the tandem purification of RGA, utilizing the 6x His- and 3x FLAG-tags, resulted in relatively pure RGA samples in all preps, indicated by $>90 \%$ of the base peak chromatogram belonging to RGA peptide signal. While neither digest could exclusively achieve such a high level of coverage, the combination of peptides detected between trypsin and AspN digests yielded 91\% sequence coverage of RGA (Figure 2.21)

\section{RGA Sequence Coverage}

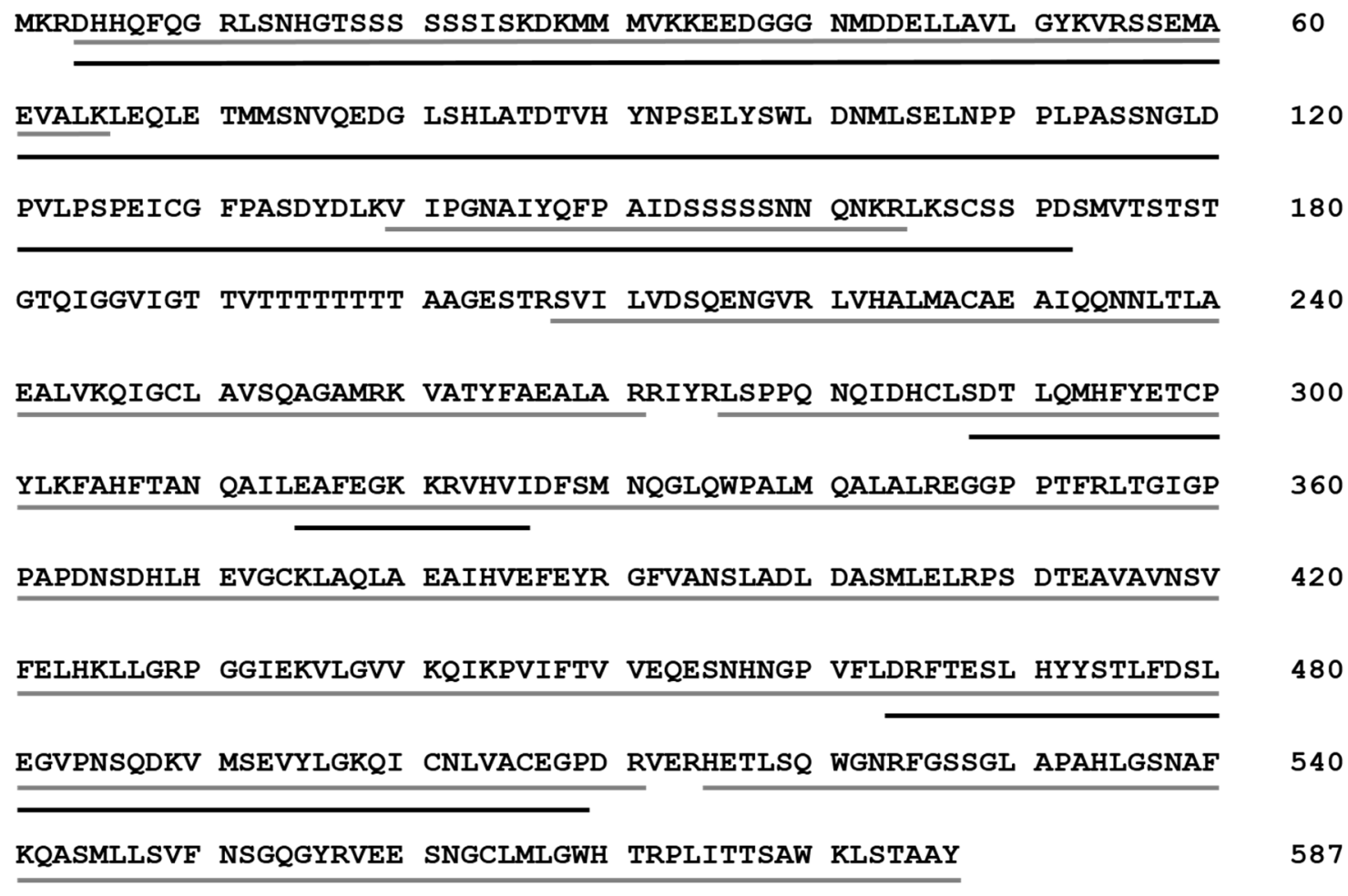

Figure 2.21 - RGA Sequence coverage from trypsin (gray) and AspN (black) digests totaled 91\% including $74 \%$ of Ser and Thr residues.

Still, only $74 \%$ of all Ser and Thr residues were detected. This was because the tryptic peptide, SCSSPDSMVTSTSTGTQIGGVIGTTVTTTTTTTTAAGESTR, and the AspN peptide, DSMVTSTSTGTQIGGVIGTTVTTTTTTTTTAAGESTRSVILV, contain two Ser/Thr-rich domains. Neither peptide covering this poly-Ser/Thr region was detected. In order to probe the efficiency of digestion in this region, the flanking peptides in the tryptic digest of RGA were examined. The 
$\mathrm{N}$-terminally and C-terminally flanking peptides, VIPGNAIYQFPAIDSSSSSNNQNKR and

SVILVDSQENGVR respectively, were both detected at $\sim 100 \%$ level relative to the most abundant RGA peptides detected as a whole, demonstrating that trypsin targeted and cleaved this region successively and the entire population of these peptides were accounted for and identified. Detection of this region remained a high priority as it contained $19 \mathrm{Ser} / \mathrm{Thr}$ residues and was central to the hypothesis that RGA co-expressed with SEC is heavily O-GIcNAcylated.

Detection of the S/T-rich domain by alternative digests

To further examine the potential success of alternative methods to detect the missing Ser/Thr-rich domain, recombinant 6x His- and 3x FLAG-tagged RGA expressed in E. coli was used. RGA bound to anti-FLAG agarose beads was reduced and alkylated with DTT and IAA as described previously, but with the addition of $4 \mathrm{M}$ urea to the buffer to encourage further RGA denaturation and increase protease accessibility. RGA was cleaved C-terminally to its lysine residues by endoproteinase $\mathrm{LySC}$, an enzyme that retains activity in high concentrations of urea. The resulting peptides were extracted, diluted to $0.5 \mathrm{M}$ urea (amenable to trypsin), and subdigested with trypsin to generate the peptide sequence, SCSSPDSMVTSTSTGTQIGGVIGTTVTTTTTTTTAAGESTR. The digest as a whole remained similar in efficiency to that from the tobacco sample. Still, SCSSPDSMVTSTSTGTQIGGVIGTTVTTTTTTTTAAGESTR was now detected in the analysis of this digest, but only at $<5 \%$ abundance relative to the RGA peptides as a whole and with a very poor elution profile, proving that the peptide was surprisingly retentive despite a heavy concentration of hydroxyl groups and polar residues, tailing for longer than 20 min under high organic phase. If modified forms of this peptide present at $<10 \%$ were to elute in this manner, they would fall below the detectable dynamic range of the mass spectrometer. Moreover, the 
same peptide was again undetectable when applying this method to the RGA (+SEC) sample

expressed in tobacco, providing further evidence that protease accessibility and efficiency was sufficient, so urea was not necessary for digestion. However, this observation was consistent with the hypothesis that the Ser/Thr-rich region could be heavily O-GlcNAcylated, because if the peptide existed in several different modified forms (i.e. different masses), the ion current for each species would be reduced relative to the solely unmodified form present in the recombinant sample, and this could cause all RGA (+SEC) forms to fall below the limit of detection. An AspN digestion, conducted in the absence of urea, yielded DSMVTSTSTGTQIGGVIGTTVTTTTTTTTAAGESTRSVILV as well, but it suffered from the same poor elution and abundance as the peptide generated in the same region by trypsin. This peptide was further sub-digested with endoproteinase Glu-C, cleaving C-terminally to glutamic acid, with the idea that the shorter DSMVTSTSTGTQIGGVIGTTVTTTTTTTTAAGE peptide may exhibit more hydrophilicity and adopt a sharper elution profile. Unfortunately, the peptide was no longer detected. One may reason that in actuality, the absence of the positively-charged Arg had the opposite of the intended chromatographic effect. Accordingly, tryptic RGA peptides were subdigested with AspN to generate the similar DSMVTSTSTGTQIGGVIGTTVTTTTTTTTAAGESTR peptide still retaining the basic Arg on the C-terminus. The peptide was once again detected but with no improvement on elution profile or sensitivity. (Figures 2.22A and 2.22B) give representative XICs of the Ser/Thr-rich domain when using alternative chromatography stationary phases, digests, and chemical derivatization. 

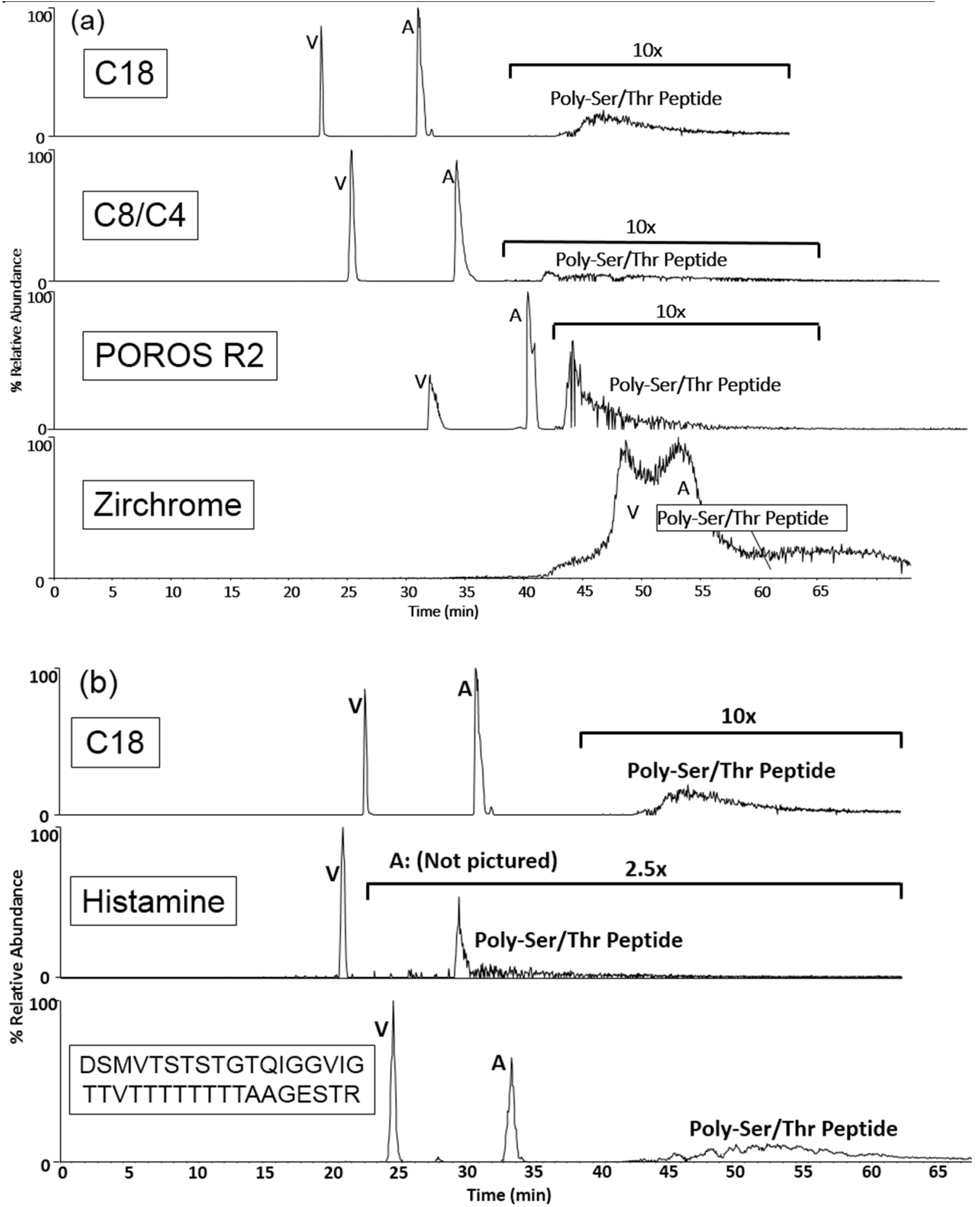

Figure 2.22-XICs of Angiotensin (A) and Vasactive Intestinal Peptide (V) internal standards and Poly-Ser/Thr peptide SCSSPDSMVTSTSTGTQIGGVIGTTVTTTTTTTTAAGESTR separated by (a) four different chromatographic stationary phases, and (b) C18 after histamine derivatization or Trypsin+AspN digestion. 
Detection of the $S / T$-rich domain by alternative stationary phases

Several alternative chromatographic stationary phases were chosen to potentially improve the chromatographic profile of the tryptic peptide, SCSSPDSMVTSTSTGTQIGGVIGTTVTTTTTTTTAAGESTR, by decreasing band broadening, tailing, and overall retention. Octyl (C8) and butyl (C4) carbon chains were employed for packing of a PC and $A C$, respectively. $\mathrm{C} 8$, less hydrophobic in nature relative to $\mathrm{C} 18$, is traditionally employed for separation of especially hydrophobic peptides, with the even less retentive C4 generally being applied to whole proteins. This setup used $\mathrm{C} 8$ for loading and binding of the target hydrophobic peptide, and C4 for analytical separation. A separate ACPC setup was packed with POROS $^{\circledR}$ R2 (Invitrogen) a Styrene-Divinylbenzene polymer phase, sometimes used for biomolecules as an alternative base support to silica-supported carbon chains ${ }^{59}$. POROS ${ }^{\circledR}$ R2 is not further derivatized and is marketed to be suitable for peptides. A third type of stationary media, Zirchrome ${ }^{\circledR}$-CARB, was selected for its reported use in separations of oligosaccharide isomers by its graphitized carbon-coated surface on $\mathrm{ZrO}_{2}{ }^{60}$. An AC and PC were both packed with Zirchrome ${ }^{\circledR}$-CARB with the hypothesis that if carbohydrates eluted effectively, the density of hydroxyls on and potential glycosylation of the target tryptic peptide may give it the appropriate binding affinity to do the same. However, none of the alternate stationary phase setups improved the elution profile and recovery of the target peptide by reverse-phase chromatography.

Detection of the S/T-rich domain by HILIC

One alternative to reverse-phase chromatography is hydrophilic interaction liquid chromatography (HILIC), a technique comparable to normal-phase in which a polar stationary phase is employed. Although the mechanism of separation is not fully understood, it has been 
empirically demonstrated that the most hydrophilic residues on peptides tend to contribute the most to retention while the most hydrophobic have little effect or even demote retention ${ }^{61,62}$. One disadvantage observed is that peptides do not ionize as efficiently sprayed under HILIC mobile phase relative to the aqueous dilute acid employed for reverse-phase and sensitivity suffers as a result. Peptides were loaded onto a C18 micro-capillary column and desalted with $0.1 \%$ acetic acid prior to HILIC, because the influence from ionic species could have a significant and undesirable effect on HILIC chromatography and load retention. Though this method of desalting is commonly used for purposes of desalting small amounts (pmols-fmols) of peptides with $>50 \%$ recovery, it was already known that the peptide in question did not elute with high recovery from $\mathrm{C} 18$ by acetonitrile. In an attempt to bypass this problem, the desalted peptides were eluted using an $80 \%$ 2-propanol solution as 2-propanol is commonly used as a solvent to stringently clean and regenerate reverse-phase packing material. Unfortunately, a screen of the eluent revealed no peptides were recovered from this cleanup.

Detection of the S/T-rich domain by Derivatization of Carboxylic Acids with Histamine

Finally, a chemical derivatization strategy of derivatizing carboxylic acid residues (Asp, Glu, and C-terminus) with histamine was implemented. This technique was developed by Dr. Michelle English in the Hunt Laboratory with the original purpose of increasing peptide basicity thereby increasing protonation and charge to improve ETD fragmentation ${ }^{45}$. Another consistent effect of this derivatization is decreased retention under reverse-phase, which could potentially be exploited to enhance detection. Improving upon similar techniques ${ }^{63-65}$, carboxylic acids on peptides were acid amidated with excess histamine using the water-soluble 1-ethyl-3-(3-dimethylaminopropyl)carbodiimide (EDC) coupling reagent in $1 \mathrm{M}$ pyridine- $\mathrm{HCl}(\mathrm{pH}$ 
5-5.5) (Figure 2.23) as described in ${ }^{45}$.
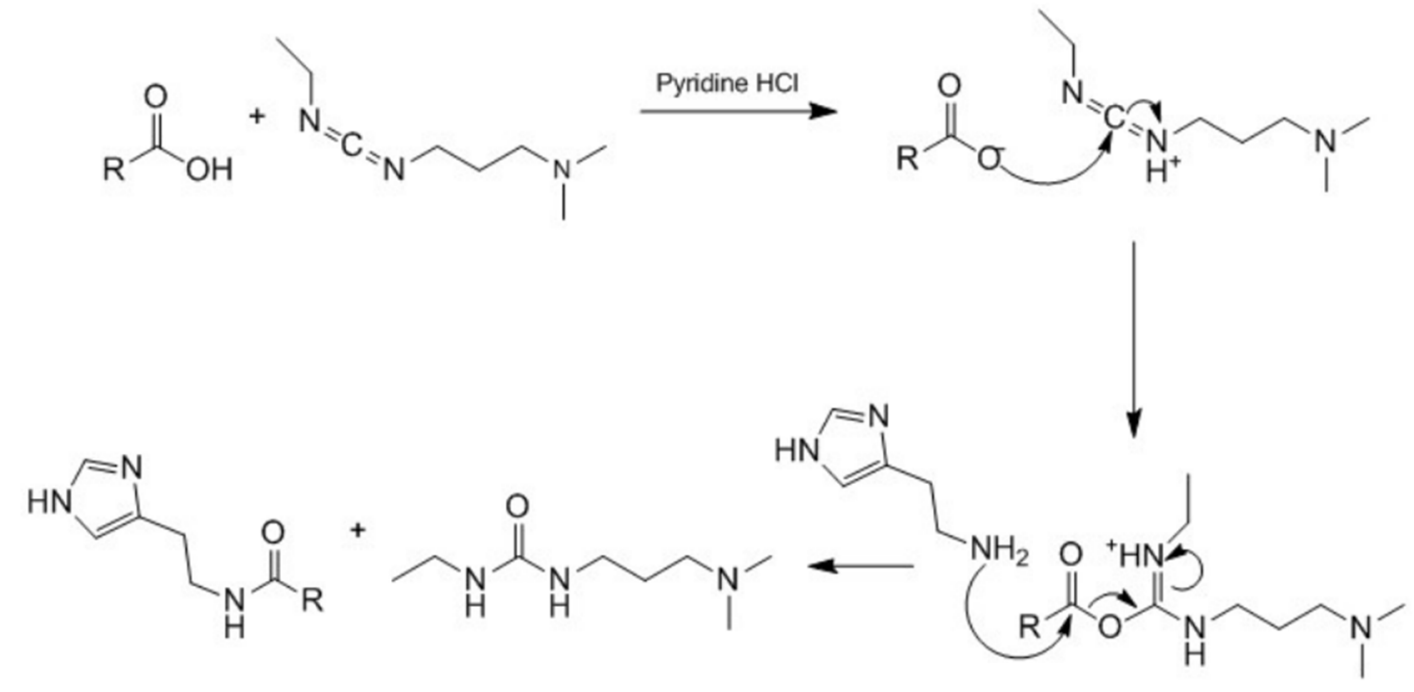

Fiqure 2.23 - Reaction mechanism for derivatization of carboxylic acids with histamine.

Peptides are derivatized and loaded directly onto C18 columns as the pyridine buffer, histamine, and urea-like byproduct washed through the column. This minimizes the need for further sample handling and potential peptide loss.

SCSSPDSMVTSTSTGTQIGGVIGTTVTTTTTTTTAAGESTR contained 3 carboxylic acid-containing residues (bolded) to be modified. The peptide was fully derivatized with 3 histamines to $50 \%$, decreasing retention on $\mathrm{C} 18$ by 15 min while moderately mitigating tailing. This yielded moderate improvement in chromatographic profile, but sensitivity remained poor.

Detection of the S/T-rich domain by Lysine Insertion

All generated forms of the Ser/Thr-rich region were inadequate as a platform for OGlcNAc detection and site-mapping due to irreversible binding, extreme chromatographic tailing, and/or poor ionization. Because of this, we employed a site-directed mutagenesis strategy to generate a new tryptic cleavage site that would break the peptide into two relatively 
equal halves. A new $6 x$ His $3 x$ FLAG-RGA vector was expressed containing a lysine insertion

(bolded) between G185 and G186: SCSSPDSMVTSTSTGTQIGKGVIGTTVTTTTTTTTAAGESTR. This site was chosen because it was located in the middle of the longest continuous sequence of residues not containing Ser or Thr and because it would be adjacent to two glycine residues.

Glycine is unique amongst the amino acids as it only contains a hydrogen for a side chain, giving it unique flexibility and the least and $4^{\text {th }}$-least propensity to participate in common secondary structures such as alpha helices and beta sheets respectively, as empirically classified by Chou and Fasman ${ }^{66}$. Because of these factors, this lysine insertion site was hypothesized to be the least likely to cause disruption in any potential RGA secondary or tertiary structure or SEC binding. The 6x His 3x FLAG RGA-G185_G186insK was over-expressed with SEC in tobacco as previously described. Western Blot analysis demonstrated that the decreased mobility of RGA (+SEC) by gel electrophoresis was characteristic of RGA-G185_G186insK as well (Figure 2.24).

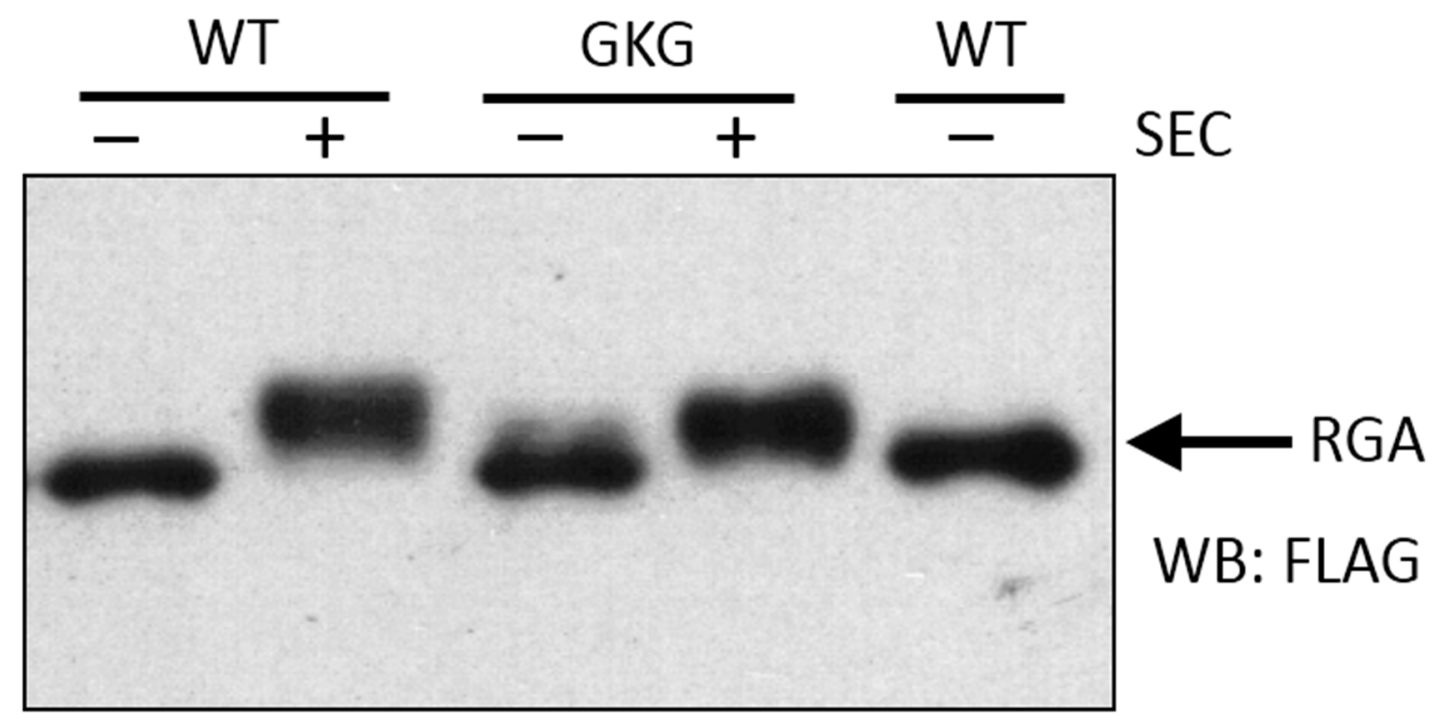

Figure 2.24 - Western Blot with anti-FLAG-HRP of RGA run on 6\% acrylamide SDS-PAGE demonstrated that the shift characteristic of RGA(+SEC) still remained for RGA-G185_G186insK (+SEC) samples. 
Following reduction, alkylation, and trypsin-digestion, peptides were separated by C18 and analyzed my MS. Contrary to their intact counterpart from RGA (+SEC), peptides SCSSPDSMVTSTSTGTQIGK and GVIGTTVTTTTTTTTAAGESTR were now both detected with a 10+ min decrease in retention, sharp symmetric peak shape for the former, and markedly improved peak shape for the latter.

\subsubsection{Posttranslational Modification of RGA(+SEC)}

Detection of RGA O-GICNAcylation

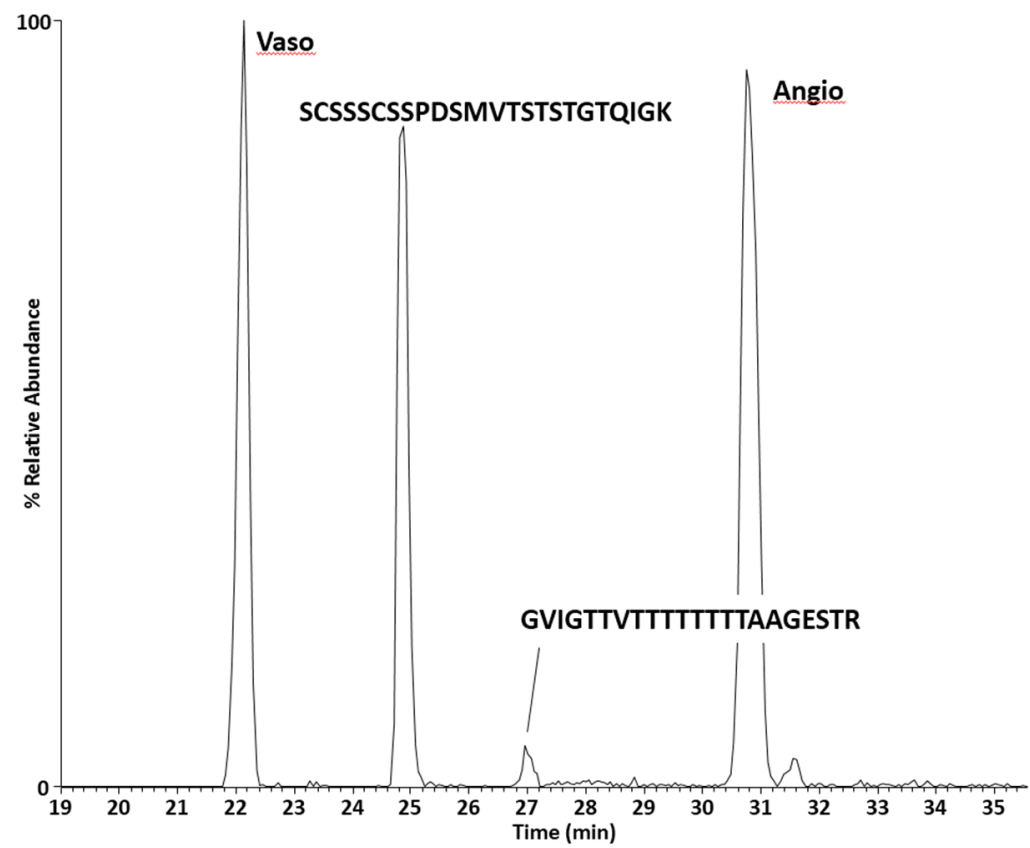

Figure 2.25 -XICS of the previously undetectable RGA(+SEC) poly-Ser/Thr region eluting as two peptides amongst peptide standards Vaso and Angio after trypsin cleaved it in half at the inserted lysine.

Through OMSSA-assisted data searches and manual searches and interpretation of $\mathrm{MS}^{1}$ and $\mathrm{MS}^{2}$ spectra, 13 unique O-GIcNAcylated peptides were discovered on RGA and RGAG185_G186insK (+SEC). These were comprised of 6 AspN-generated and 7 trypsin-generated peptides, including SCSSPDSMVTSTSTGTQIGK and GVIGTTVTTTTTTTTAAGESTR, containing a dense population of Ser and Thr residues hypothesized to be heavily O-GIcNAcylated. Owing to 
the lysine insertion creating a new tryptic cleavage site, these modifiable residues were now detected by mass spectrometry (Figure 2.25) and mass spectral analysis determined that SCSSPDSMVTSTSTGTQIGK contained one, two, and three simultaneously occurring O-GICNAC sites while GVIGTTVTTTTTTTTAAGESTR contained all combinations of one through six coinciding O-GlcNAc sites (Figure 2.26 ).

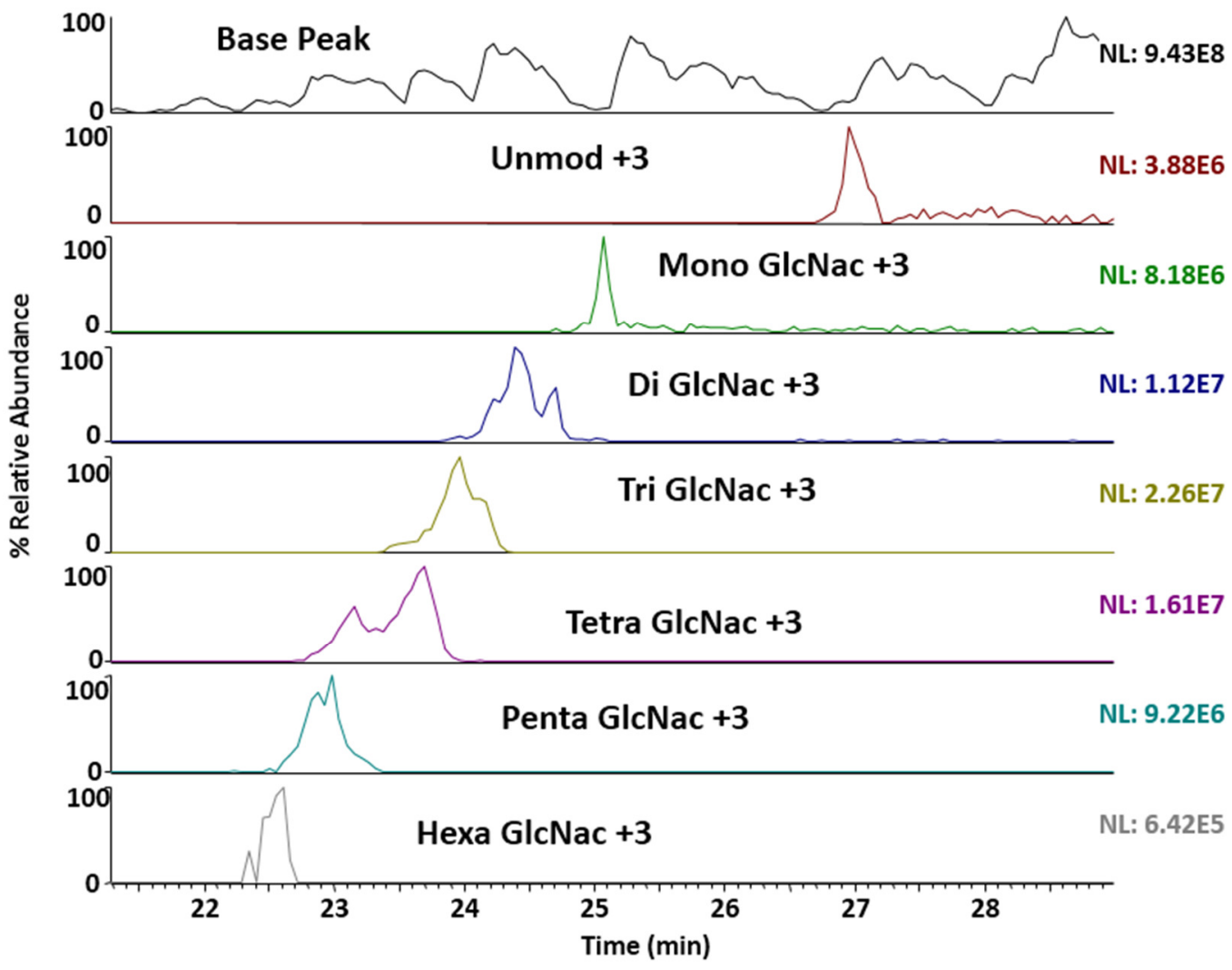

Figure 2.26 - XICS of 1-6 O-GICNAcs detected on GVIGTTVTTTTTTTTAAGESTR.

The two peptides comprised of the remaining two RGA regions that contain $>3$ sequential Ser/Thr residues were also found to contain O-GlcNAc. Asp-N-generated peptide DHHQFQGRLSNHGTSSSSSSISK was mono-, di-, tri-, and tetra-O-GIcNAcylated while tryptic peptide VIPGNAIYQFPAIDSSSSSNNQNKR was mono-O-GIcNAcylated. Outside of these polySer/Thr domains, an additional nine regions of O-GIcNAcylation were detected, two of which also bore multiple simultaneous O-GlcNAcs. 
Detection of RGA Phosphorylation

Yet, O-GIcNAcylation was not the only PTM detected on RGA. Of the 13 O-GlcNAcylated peptides detected, 4 also contained phosphorylated forms. It was previously unknown that RGA could be phosphorylated, but not entirely unexpected as an estimated $1 / 3$ of the proteome may be phosphorylated and the vast majority O-GlcNAcylated proteins are also phosphorylated at some point during their lifetimes, whether separately from or simultaneously with O-

GlcNAcylation ${ }^{3,7}$. While it was known that one or more tobacco kinases were responsible for this phosphorylation of RGA, the exact kinases responsible were unknown. Interestingly, three of the four phosphorylated peptides contained poly-Ser/Thr domains.

DHHQFQGRLSNHGTSSSSSSISK contained mono-, di-, and tri-phosphorylation while DPVLPSPEICGFPAS, VIPGNAIYQFPAIDSSSSSNNQNKR, and SCSSPDSMVTSTSTGTQIGK contained mono-phosphorylation. Of the poly-Ser/Thr containing RGA peptides generated, only GVIGTTVTTTTTTTTAAAGESTR contained no detectable phosphorylation.

Detection of an unexpected and unprecedented glyco-PTM

By examining $\mathrm{MS}^{1}$ spectra, it was observed that several species with mass shifts of $\Delta 41$ Da were co-eluting with the O-GIcNAcylated forms of GVIGTTVTTTTTTTTAAGESTR (Figure 2.27). 


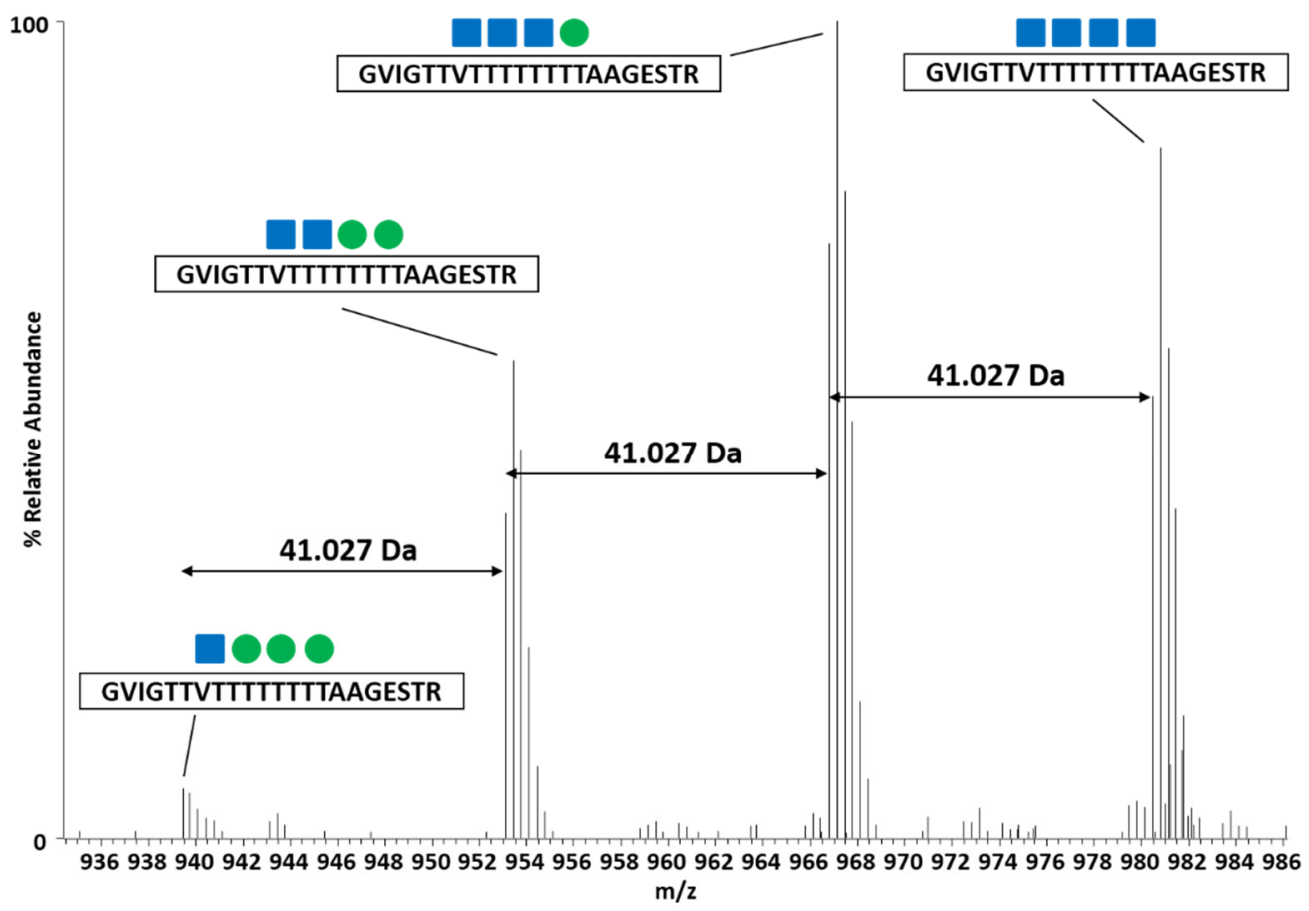

Figure 2.27 - Masses related to O-GlcNAcylated poly-Thr/Ser domain were due to a previously undescribed nuclear protein PTM, O-hexosylation. O-GICNAcylation and O-hexosylation are indicated by blue squares and green circles, respectively.

Interestingly, no such mass shift occurred relative to the unmodified peptide form. Given their similar mass, charge states, isotopic distribution, abundance, and retention, these $\Delta 41 \mathrm{Da}$ shifted-peptides were further investigated for their potential relationship to the O-GlcNAcylated peptides. Because the intact peptides were detected in the Orbitrap with high resolution and accurate mass, the mass shifts were precisely calculated to $41.027 \mathrm{Da}$. While this $\Delta$ mass pertained to two known theoretical modifications (i.e. Ser>Gln substitution and amidination of lysines or N-terminal amines with methyl acetimidate ${ }^{67}$, neither of these possibilities fit the circumstances. The number of $\Delta 41 \mathrm{Da}$ shifts on numerous single peptides outnumbered the single Ser present on GVIGTTVTTTTTTTTAAGESTR and the sample was never treated with methyl acetimidate (Figure 2.28). Given that the shift occurred only in relation to O-GlcNAcylated forms, $\Delta 41$ Da was examined relative to O-GlcNAc itself. In fact, a loss of 41 Da equated to the 


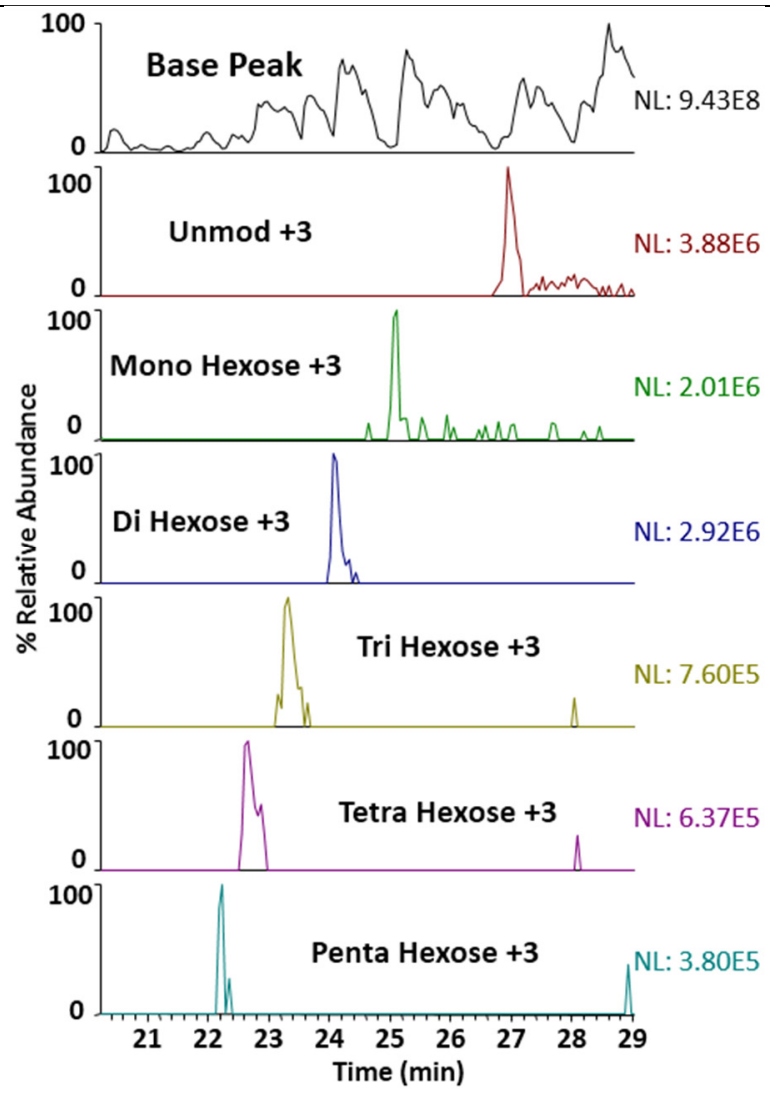

Figure 2.28 - XICS of 1-5 unexpected $\triangle 162$ masses on GVIGTTVTTTTTTTTAAGESTR determined to be O-hexose PTMs.

mass difference ( $\triangle 41.0266 \mathrm{Da}$ ) between GlcNAc and a regular hexose sugar (potentially glucose) lacking the $\mathrm{N}$-acetyl moiety.

A full analysis of the data revealed that in total, peptides comprised of six distinct regions of RGA contained one or more hexose moieties. All six of these regions also contained O-GIcNAcylated peptide forms and of these, two were modifiable with all three PTMs found in this study. CAD of hexosylated peptides generated signature ions from the loss of the hexose (Figure 2.29), indicating they were O-linked analogous to O-GlcNAc. Several hexose sites were able to be localized by ETD and unambiguously verified to contain O-linkages to Ser or Thr residues. In all cases, O-hexose was detected as a monosaccharide PTM only, with no elongation. 


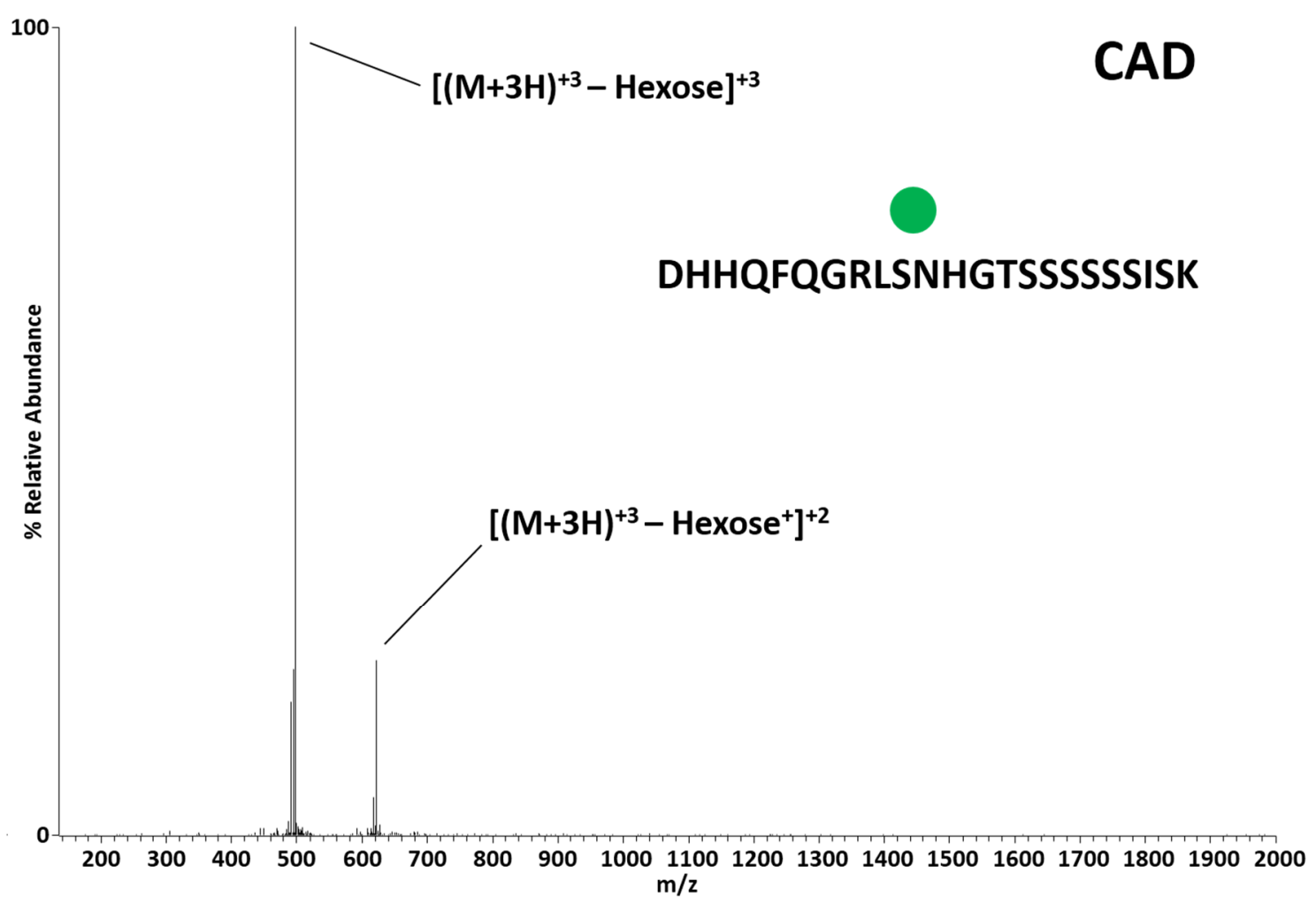

Figure 2.29 - Hexose PTMs also produced signature fragment ions upon CAD, characteristic of O-linkage. This spectrum indicates that mono-O-hexosylation is present within RGA residues 4-26.

Prior to the discovery of O-GIcNAc, it was widely believed that nuclear (and cytoplasmic) proteins contained no glycosylation at all. Reports of glycosylation of nuclear and cytoplasmic proteins apart from O-GIcNAcylation remain a minority in the literature, yet there is a growing body of evidence supporting additional glycosylation of nucleocytoplasmic proteins and the nucleocytoplasmic domains of transmembrane proteins ${ }^{68}$. Most of these studies have used lectins (sugar-binding proteins) or anticarbohydrate antibodies to probe for these alleged glycoproteins. These results can be difficult to validate though due to the lack of structural data confirming the glycans and the capability for lectins and antibodies to bind to biomolecules nonspecifically (e.g. via hydrophobic interactions). Nucleocytoplasmic glycosylation is also counterintuitive given the present understanding that most glycosyltransferases are membrane 
proteins whose active sites are located within the lumen of the Endoplasmic Reticulum (ER) or

Golgi. A few possibilities exist to justify the existence of these reports. There is a possibility that unknown and unconventional glycosyltransferases reside in the cytoplasm and/or nucleus. There are indeed reports of glycosyltransferase activity in highly purified rat liver nuclei, although even minor contamination with the ER could yield misleading results. Alternatively, some proteins have been found to relocate to the cytoplasm after entering the ER and there is evidence that endocytosed glycoproteins may enter the cytoplasm in this way. For a third possibility, some membrane-associated glycosyltransferases' are located on the cytoplasmic side of the ER and are responsible for the synthesis of glycolipids and dolichyl-linked N-glycosylation precursors that are afterwards transported into the ER lumen for attachment to proteins. It is conceivable that one or more of these glycoproteins may have developed the capability to glycosylate proteins located in the cytoplasm ${ }^{68}$.

Despite these limited reports of other nucleocytoplasmic glycosylation, the detection of an O-linked hexose PTM on RGA was unexpected for two reasons: (1) it is a nuclear protein and O-GIcNAcylation is the only nuclear protein glyco-PTM that has been rigorously validated and widely accepted as discussed above, and (2) simple O-linked monosaccharide glyco-PTMs apart from O-GIcNAc are virtually undocumented regardless of cellular or extracellular location.

A few reported exceptions to this occur for the glycosylation of Drosophila and mammalian proteins, containing epidermal growth factor (EGF)-like repeat domains. These EGF repeats consist of 30-40 amino acids including 6 conserved cysteine residues that form 3 intramolecular disulfide bonds ${ }^{69}$. About 25 years ago, the atypical O-linked glycol modification O-glucose was discovered as a trisaccharide sugar chain linked to Ser52 in bovine blood coagulation factors VII and IX ${ }^{70}$. Soon after, the atypical O-linked O-fucose was detected and 
localized to the nearby residue, Ser60, on human blood coagulation factor VII (O-glucose

oligosaccharide was also again detected on Ser52 in this study) ${ }^{71}$. In the same time period, Ofucose was also detected on factor $\mathrm{XII}^{72}$ and human Prourokinase, a serine protease first discovered in urine ${ }^{73}$. Later, both modifications were also discovered on the extracellular EGFdomains of Notch1, one of four mammalian notch proteins important for cellular signaling ${ }^{74}$. In all cases, O-fucose maintained the consensus site of Cys $2-\mathrm{X}-\mathrm{X}-\mathrm{Gly}-\mathrm{Gly}-\mathrm{Ser} / \mathrm{Thr}-\mathrm{Cys}_{3}$ where the site of modification is indicated on the bolded Ser or Thr between the $2^{\text {nd }}$ and $3^{\text {rd }}$ Cys residue of the EGF repeat and $\mathrm{X}$ is any residue. Depending on the clotting factor, it was determined to exist as either a monosaccharide or oligosaccharide modification, and was detected in both forms on Notch1. O-glucose held the consensus sequence Cys $1-X-S e r-X-P r o-C y s_{2}$ where the site of modification is indicated on the bolded Ser between the $1^{\text {st }}$ and $2^{\text {nd }}$ Cys residue of the EGF repeat and $\mathrm{X}$ is any residue. It was also detected as both a monosaccharide and oligosaccharide on Notch1, but was further elongated on all clotting factors described. Many additional Oglucose and O-fucose glycosylation sites have been discovered on Notch protein, mainly from studies using Drosophila, with the modifications all adhering to their previously characterized consensus motifs ${ }^{75,76}$. These rare glyco-modifications are interesting in light of the Ohexosylation discovered by this study of RGA, especially as O-glucose is an O-hexose modification. However, these literature modifications differ from RGA O-hexose in a few significant ways: (1) Notch and coagulation factors are restricted to metazoans, (2) these proteins are modified on consensus sequences of EGF domains by glycosyltransferases in the ER or Golgi, (3) the modified domains are extracellularly located, and (4) the monosaccharide modifications can still be elongated into oligosaccharides in many cases. It is worth noting that Notch proteins also contain the only documented sites of extracellular O-GIcNAc, the addition of which is catalyzed by an epidermal O-GIcNAc transferase (EOGT) in both Drosophila ${ }^{77}$ and 
mammals ${ }^{78}$. In both cases O-GIcNAc was mapped to a Thr residue located between the $5^{\text {th }}$ and $6^{\text {th }}$ Cys residues of EGF repeats, and evidence suggests that it may be elongated at least in mammals. This EOGT is distinct from what is traditionally referred to as OGT, still the only known enzyme responsible for catalyzing the addition of O-GIcNAc to nucleocytoplasmic proteins of metazoans. For the reasons described above, it is reasonable to conclude that like EOGT and nucleocytoplasmic O-GIcNAcylation, the glycosyltransferases responsible for O-fucose and O-glucose are entirely separate from what is responsible for the O-hexosylation of RGA described in this dissertation. This new O-hexose is unprecedented for the fact that it resides on a nuclear protein and one that is expressed by plants.

Stoichiometry and Site-locations of RGA O-GIcNAcylation, Phosphorylation, and O-hexosylation An in-depth analysis of RGA PTMs revealed 23 O-GIcNAc, 6 phosphate, and 14 O-hexose sites based on the detection of these modifications on intact peptides ( $\mathrm{MS}^{1}$ ) and ETD fragment ions $\left(\mathrm{MS}^{2}\right)$. For reasons previously discussed, fragmentation by ETD preserved all three of these labile PTMs during $\mathrm{MS}^{2}$ and enabled the majority of these sites to be localized to the exact residue modified as summarized in TABLE 2.3. 


\begin{tabular}{|c|c|c|c|}
\hline 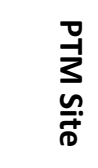 & 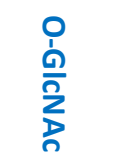 & 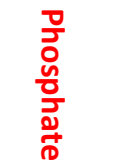 & 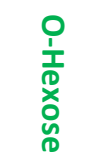 \\
\hline S13 & & $x$ & \\
\hline T17 & $x$ & $x$ & $x$ \\
\hline S18 & $x$ & & $x$ \\
\hline S19 & $x$ & & $x$ \\
\hline S20 & $x$ & $x$ & $x$ \\
\hline S23 & & $x$ & \\
\hline S56 & $x$ & & $x$ \\
\hline S57 & $x$ & & $x$ \\
\hline S82 & $x$ & & $x$ \\
\hline S115 & $x$ & & $x$ \\
\hline S116 & $x$ & & $x$ \\
\hline S170 & & $x$ & \\
\hline T176 & $x$ & & \\
\hline T177 & $x$ & & \\
\hline T178 & $x$ & & \\
\hline S253 & $x$ & & \\
\hline S537 & $x$ & & \\
\hline & Total & Total & Total \\
\hline & 14 & 5 & 9 \\
\hline
\end{tabular}

Table 2.3 - 14 O-GICNAC, 5 phosphate, and 9 O-hexose sites were unambiguously localized to the following Ser or Thr residues as indicated by " $X$ ".

Peptides were observed to be modified in a wide range of stoichiometries ( $<1 \%$ to $>50 \%)$ and to various degrees of combinatorial PTM complexity including the presence of all three modifications at once. A summary of all modified peptides and corresponding PTM sites detected, totaling 89 distinct forms in all, is presented in Table $\mathbf{2 . 4}$. 
Chapter 2

PTM Analysis of RGA

79

\begin{tabular}{|c|c|c|c|c|c|c|c|c|c|c|c|c|c|}
\hline \multirow{2}{*}{ Residues } & \multirow{2}{*}{ Peptide sequence } & \multicolumn{3}{|c|}{ \# Mods } & \multirow{2}{*}{$\frac{\frac{n}{\tilde{D}}}{\underline{\underline{D}}}$} & \multirow{2}{*}{$\frac{7}{3}$} & \multirow{2}{*}{$\mathbf{z}$} & \multirow{2}{*}{ 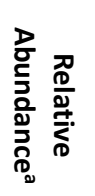 } & \multicolumn{3}{|c|}{ Digest } & & \multirow{2}{*}{ 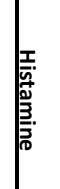 } \\
\hline & & & & & & & & & Trypsin & AspN & CNBr & & \\
\hline 4-26 & DHHQFQGRLSNHGTgSSgSSSSISK & 1 & & & S18 \& S20 & 0.3 & 5 & $\begin{array}{c}++++ \\
+\end{array}$ & & $\mathrm{x}$ & & & \\
\hline 4-26 & DHHQFQGRLSNHGg[TgSgSSS]SSISK & 2 & & & $S 18,19, \&[]^{d}$ & 0.1 & 5 & ++++ & & $\mathrm{x}$ & & & \\
\hline 4-26 & DHHQFQGRLSNHGgTgSg[SSS]SSISK & 3 & & & $\mathrm{~T} 17, \mathrm{~S} 18, \&[]^{\mathrm{d}}$ & $\begin{array}{c}<0 \\
1\end{array}$ & 5 & ++ & & $\mathrm{x}$ & & & \\
\hline $4-26$ & $\begin{array}{c}\text { DHHQFQGRLgggg(SNHGTSSSSSSIS) } \\
\qquad\end{array}$ & 4 & & & bc & 0.4 & 5 & + & & $\mathrm{x}$ & & & \\
\hline 4-26 & DHHQFQGRLPSNHGp[TSSS]SSSISK & & 1 & & $S 13 \&[]^{d}$ & 0.4 & 4 & + & & $\mathrm{x}$ & & & \\
\hline 4-26 & DHHQFQGRLPSNHGp[TSSpSSSSIS]K & & 2 & & S13 \& S20 & 0.3 & 4 & + & & $\mathrm{x}$ & & $x$ & \\
\hline $4-26$ & DHHQFOGRLppp(SNHGTSSSSSSIS)K & & 3 & & bc & 0.7 & 4 & + & & $\mathrm{x}$ & & & \\
\hline 4-26 & DHHQFQGRLSNHGThSSSSSSISK & & & 1 & s18 & 0.5 & 5 & ++ & & $\mathrm{x}$ & & & \\
\hline $4-26$ & DHHQFOGRLSNHGh[TSS]SSSSISK & & & 1 & d & 0.9 & 6 & ++ & & $\mathrm{x}$ & & & $\mathrm{x}$ \\
\hline 4-26 & DHHQFQGRLSNHGThShSSSSSISK & & & 2 & S18 \& S19 & 0.6 & 5 & + & & $\mathrm{x}$ & & & \\
\hline $4-26$ & DHHQFQGRLhhh(SNHGTSSSSSSIS)K & & & 3 & bc & 1.3 & 5 & + & & $\mathrm{x}$ & & & \\
\hline $4-26$ & DHHQFQGRLPSNHGgTgSgSSSSSISK & 1 & 1 & & $\begin{array}{l}\mathrm{pS} 13 \text { \& gT17, } \\
\mathrm{gS} 18, \mathrm{gS} 19\end{array}$ & $\begin{array}{c}<0 \\
1\end{array}$ & 5 & ++ & & $\mathrm{x}$ & & $\mathrm{x}$ & \\
\hline 4-26 & $\begin{array}{l}\text { DHHQFQGRLPSNHGpg[TSSS]SSpSIS } \\
\qquad \mathrm{K}\end{array}$ & 1 & 2 & & $\begin{array}{l}\mathrm{pS} 13, \mathrm{gp}[]^{\mathrm{d}}, \& \\
\mathrm{pS} 23\end{array}$ & 0.8 & 4 & + & & $\mathrm{x}$ & & $\mathrm{x}$ & \\
\hline $4-26$ & $\begin{array}{l}\text { DHHQFQGRLPSNHGpg[TSSSSS]pSIS } \\
\qquad \mathrm{K}\end{array}$ & 1 & 3 & & $\begin{array}{l}\mathrm{pS} 13, \mathrm{gp}[]^{\mathrm{d}}, \& \\
\mathrm{pS} 23\end{array}$ & 1.6 & 4 & + & & $\mathrm{x}$ & & $\mathrm{x}$ & \\
\hline $4-26$ & $\begin{array}{l}\text { DHHQFQGRLpSNHGpgTgSgSSSSSIS } \\
\qquad \mathrm{K}\end{array}$ & 2 & 1 & & $\begin{array}{l}\text { pS13, pgT17, } \\
\text { gS18, \& gS19 }\end{array}$ & 0.7 & 5 & ++ & & $x$ & & & \\
\hline $4-26$ & $\begin{array}{c}\text { DHHQFQGRLgggp(SNHGTSSSSSSIS) } \\
\text { K }\end{array}$ & 3 & 1 & & bc & 3.7 & 5 & + & & $X$ & & & \\
\hline $4-26$ & $\begin{array}{c}\begin{array}{c}\text { DHHQFQGRLggpp(SNHGTSSSSSSIS) } \\
\mathrm{K}\end{array} \\
\end{array}$ & 2 & 2 & & bc & 1.0 & 4 & + & & $x$ & & & \\
\hline $4-26$ & DHHQFQGRLSNHGpgh[TSS]SSSSISK & 1 & 1 & 1 & $\operatorname{pgh}[]^{d}$ & 1.7 & 4 & + & & $x$ & & & \\
\hline $4-26$ & $\begin{array}{l}\text { DHHQFQGRLSNHGTghSghSghSSSSI } \\
\text { SK }\end{array}$ & 1 & & 1 & $\begin{array}{l}\text { ghS18, ghS19, } \\
\text { ghS20 }\end{array}$ & $\begin{array}{c}- \\
0.3\end{array}$ & 5 & ++ & & $x$ & & & \\
\hline
\end{tabular}


Chapter 2

PTM Analysis of RGA

\begin{tabular}{|c|c|c|c|c|c|c|c|c|c|c|c|c|}
\hline $4-26$ & DHHQFQGRLghh(SNHGTSSSSSSIS)K & 1 & & 2 & $\mathrm{bc}$ & $\begin{array}{c}- \\
1.1\end{array}$ & 5 & + & & $X$ & & \\
\hline $4-26$ & $\begin{array}{c}\text { DHHQFQGRLpSNHGphThShSSSSSIS } \\
\mathrm{K}\end{array}$ & & 1 & 1 & $\begin{array}{l}\text { pS13, phT17, } \\
\text { hS18, \& hS19 }\end{array}$ & $\begin{array}{c}- \\
1.5\end{array}$ & 4 & + & & $X$ & & \\
\hline $54-65$ & VRSSEMAEVALK & 1 & & & gS56 \& gS57 & $\begin{array}{c}- \\
0.3\end{array}$ & 3 & ++++ & $X$ & & & \\
\hline $54-65$ & VRSSEMAEVALK & 2 & & & gS56 \& gS57 & $\begin{array}{c}- \\
0.3\end{array}$ & 3 & + & $X$ & & & \\
\hline $44-59$ & DhistELLAVLGYKVRSShistEM-hsI & 1 & & & S56 & 0.2 & 4 & ++++ & & $X$ & $X$ & \\
\hline $44-59$ & DhistELLAVLGYKVRSShistEM-hsI & 1 & & & S57 & 0.2 & 4 & ++ & & $X$ & $X$ & \\
\hline $44-59$ & DhistELLAVLGYKVRSShistEM-hsI & & & 1 & S56 & 0.8 & 4 & ++ & & $X$ & $X$ & \\
\hline $44-59$ & DhistELLAVLGYKVRSShistEM-hsI & & & 1 & S57 & 0.8 & 4 & + & & $X$ & $X$ & \\
\hline $79-86$ & DGLgSHLAT & 1 & & & S82 & $\begin{array}{c}<0 \\
1\end{array}$ & 2 & +++ & & $X$ & & \\
\hline $79-86$ & DGLhSHLAT & & & 1 & S82 & $\begin{array}{c}- \\
2.1\end{array}$ & 2 & + & & $X$ & & \\
\hline $87-100$ & Dg(TVHYNPSELYS)WL & 1 & & & $\mathrm{bc}$ & 3.9 & 2 & + & & $X$ & & \\
\hline $120-134$ & DPVLPg(SPEICGFPAS) & 1 & & & $\mathrm{bc}$ & 2.3 & 2 & ++ & & $X$ & & \\
\hline $120-134$ & DPVLPp(SPEICGFPAS) & & 1 & & $\mathrm{bc}$ & 1.9 & 2 & + & & $X$ & & \\
\hline $104-119$ & LShistELNPPPLPASgSNGL-hist & 1 & & & gS116 & 0.1 & 3 & $\begin{array}{c}++++ \\
+\end{array}$ & & $X$ & $X$ & $\mathrm{X}$ \\
\hline $104-119$ & Lgg(ShistELNPPPLPASS)NGL-hist & 2 & & & $\mathrm{bc}$ & 0.6 & 3 & + & & $X$ & $X$ & $x$ \\
\hline $104-119$ & LShistELNPPPLPAShSNGL-hist & & & 1 & hS116 & 0. & 3 & ++++ & & $X$ & $X$ & $X$ \\
\hline $104-119$ & LShistELNPPPLPAhSgSNGL-hist & 1 & & 1 & hS115, gS116 & $\begin{array}{c}- \\
1.0\end{array}$ & 3 & ++ & & $X$ & $X$ & $\mathrm{X}$ \\
\hline $104-119$ & LShistELNPPPLPAgShSNGL-hist & 1 & & 1 & g115, hS116 & - & 3 & + & & $X$ & $X$ & $\mathrm{X}$ \\
\hline $137-171$ & $\begin{array}{l}\text { DLKVIPGNAIYQFPAIDg(SSSSSNNQN } \\
\text { KRLKSCSS)P }\end{array}$ & 1 & & & c & 2.0 & 5 & + & & $X$ & & \\
\hline $140-164$ & VIPGNAIYQFPAIDSSg(SSS)NNQNKR & 1 & & & c & 3.2 & 4 & ++ & $X$ & & & \\
\hline $140-164$ & VIPGNAIYQFPAIDp(SSSSS)NNQNKR & & 1 & & $\mathrm{bc}$ & - & 3 & + & $x$ & & & \\
\hline $153-171$ & DSSSSSNNQNKRLKg(SCSS)P & 1 & & & c & 1.1 & 3 & +++ & & $X$ & & \\
\hline $153-171$ & Dgg(SSSSSNNQNKRLKSCSS)P & 2 & & & $b c$ & 3.9 & 3 & +++ & & $X$ & & \\
\hline $153-171$ & DSSSSSNNQNKRLKp(SCSS)P & & 1 & & c & 1.0 & 3 & ++++ & & $X$ & & \\
\hline $153-171$ & Dh(SSSSSNNQNKRLKSCSS)P & & & 1 & $\mathrm{bc}$ & 1.6 & 3 & + & & $x$ & & \\
\hline $167-185$ & SCSSPDSMVgTSTSTGTQIGK & 1 & & & T176 & $\begin{array}{c}- \\
0.3\end{array}$ & 3 & +++ & $x$ & & & \\
\hline $167-185$ & SCSSPDSMVTSgTSTGTQIGK & 1 & & & T178 & $\begin{array}{c}- \\
0.3\end{array}$ & 3 & +++ & $X$ & & & \\
\hline $167-185$ & SCSSPDSMVg[TSTS]TGTQIGK & 1 & & & $\mathrm{~g}[]^{\mathrm{d}}$ & $\begin{array}{c}- \\
0.3\end{array}$ & 3 & + & $x$ & & & \\
\hline $167-185$ & g(SCSSPDS)MVTSTSTGTQIGK & 1 & & & c & - & 3 & +++ & $x$ & & & \\
\hline $167-185$ & g(SCSSPDSMVTSTSTGT)QIGK & 1 & & & $\mathrm{bc}$ & $\begin{array}{c}- \\
0.1\end{array}$ & 3 & +++ & $x$ & & & \\
\hline $167-185$ & gg(SCSSPDSMVTSTSTGT)QIGK & 2 & & & ce & 0.4 & 3 & +++ & $X$ & & & \\
\hline
\end{tabular}


Chapter 2

PTM Analysis of RGA

\begin{tabular}{|c|c|c|c|c|c|c|c|c|c|c|c|c|}
\hline $167-185$ & ggg(SCSSPDSMVTSTSTGT)QIGK & 3 & & & ce & 0.0 & 3 & ++ & $X$ & & & \\
\hline $167-185$ & SCSPSPDSMVTSTSTGTQIGK & & 1 & & S170 & 0.2 & 3 & ++ & $X$ & & & \\
\hline $167-185$ & h(SCSSPDSMVTSTSTGT)QIGK & & & 1 & bc & 0.8 & 3 & ++ & $X$ & & & \\
\hline $167-185$ & SCSpSPDSMVgTSTSTGTQIGK & 1 & 1 & & pS170 \& gT177 & $\overline{2.1}$ & 3 & ++ & $X$ & & & \\
\hline $167-185$ & pg(SCSSPDSMVTSTSTGT)QIGK & 1 & 1 & & ce & 0.6 & 3 & ++ & $X$ & & & \\
\hline $167-185$ & pgg(SCSSPDSMVTSTSTGT)QIGK & 2 & 1 & & ce & 0. & 3 & ++ & $X$ & & & \\
\hline $167-185$ & pggg(SCSSPDSMVTSTSTGT)QIGK & 3 & 1 & & ce & 0. & 3 & + & $X$ & & & \\
\hline $167-185$ & gh(SCSSPDSMVTSTSTGT)QIGK & 1 & & 1 & bc & 0.4 & 3 & ++ & $X$ & & & \\
\hline $186-207$ & GVIGg(TTVTTTTTTTTTAAGEST)R & 1 & & & ce & $\begin{array}{c}- \\
0.8\end{array}$ & 3 & ++ & $X$ & & & \\
\hline $186-207$ & GVIGgg(TTVTTTTTTTTTAAGEST)R & 2 & & & ce & $\begin{array}{c}- \\
1.0\end{array}$ & 3 & ++ & $X$ & & & \\
\hline $186-207$ & GVIGggg(TTVTTTTTTTTAAGEST)R & 3 & & & ce & $\begin{array}{c}- \\
0.7\end{array}$ & 3 & +++ & $X$ & & & \\
\hline $186-207$ & GVIGgggg(TTVTTTTTTTTTAAGEST)R & 4 & & & ce & $\begin{array}{c}- \\
1.4\end{array}$ & 3 & +++ & $X$ & & & \\
\hline $186-207$ & GVIGggggg(TTVTTTTTTTTAAGEST)R & 5 & & & ce & $\begin{array}{c}- \\
0.2\end{array}$ & 3 & ++ & $X$ & & & \\
\hline $186-207$ & $\begin{array}{c}\text { GVIGgggggg(TTVTTTTTTTTTAAGEST) } \\
\text { R }\end{array}$ & 6 & & & ce & $\begin{array}{c}- \\
0.3\end{array}$ & 3 & + & $X$ & & & \\
\hline $186-207$ & GVIGh(TTVTTTTTTTTAAGEST)R & & & 1 & bc & $\begin{array}{c}- \\
0.3\end{array}$ & 3 & + & $X$ & & & \\
\hline $186-207$ & GVIGhh(TTVTTTTTTTTTAAGEST)R & & & 2 & bc & $\begin{array}{c}- \\
0.4\end{array}$ & 3 & ++ & $X$ & & & \\
\hline $186-207$ & GVIGhhh(TTVTTTTTTTTAAAGEST)R & & & 3 & bc & $\begin{array}{c}- \\
0.3\end{array}$ & 3 & + & $X$ & & & \\
\hline $186-207$ & GVIGhhhh(TTVTTTTTTTTAAGEST)R & & & 4 & bc & $\begin{array}{c}- \\
1.2\end{array}$ & 3 & + & $X$ & & & \\
\hline $186-207$ & GVIGhhhhh(TTVTTTTTTTTTAAGEST)R & & & 5 & bc & $\begin{array}{c}- \\
1.7\end{array}$ & 3 & + & $X$ & & & \\
\hline $186-207$ & GVIGgh(TTVTTTTTTTTTAAGEST)R & 1 & & 1 & ce & $\begin{array}{c}- \\
0.8\end{array}$ & 3 & ++ & $X$ & & & \\
\hline $186-207$ & GVIGghh(TTVTTTTTTTTTAAGEST)R & 1 & & 2 & ce & 0.8 & 3 & ++ & $X$ & & & \\
\hline $186-207$ & GVIGghhh(TTVTTTTTTTTTAAGEST)R & 1 & & 3 & ce & $\begin{array}{c}- \\
0.4\end{array}$ & 3 & ++ & $\mathrm{X}$ & & & \\
\hline $186-207$ & GVIGghhhh(TTVTTTTTTTTTAAGEST)R & 1 & & 4 & ce & $\begin{array}{c}- \\
2.1\end{array}$ & 3 & ++ & $X$ & & & \\
\hline $186-207$ & $\begin{array}{c}\text { GVIGghhhhh(TTVTTTTTTTTTAAGEST) } \\
\text { R }\end{array}$ & 1 & & 5 & ce & $\begin{array}{c}- \\
0.1\end{array}$ & 3 & + & $X$ & & & \\
\hline $186-207$ & GVIGggh(TTVTTTTTTTTTAAGEST)R & 2 & & 1 & ce & $\begin{array}{c}- \\
0.5\end{array}$ & 3 & +++ & $\mathrm{X}$ & & & \\
\hline $186-207$ & GVIGgghh(TTVTTTTTTTTTAAGEST)R & 2 & & 2 & ce & $\begin{array}{c}- \\
0.8\end{array}$ & 3 & +++ & $X$ & & & \\
\hline $186-207$ & GVIGgghhh(TTVTTTTTTTTTAAGEST)R & 2 & & 3 & ce & $\begin{array}{c}- \\
1.9\end{array}$ & 3 & ++ & $X$ & & & \\
\hline $186-207$ & $\begin{array}{c}\text { GVIGgghhhh(TTVTTTTTTTTTAAGEST) } \\
\text { R }\end{array}$ & 2 & & 4 & ce & $\begin{array}{c}- \\
1.1\end{array}$ & 3 & + & $X$ & & & \\
\hline $186-207$ & GVIGgggh(TTVTTTTTTTTTAAGEST)R & 3 & & 1 & ce & $\begin{array}{c}- \\
1.0\end{array}$ & 3 & +++ & $X$ & & & \\
\hline $186-207$ & GVIGggghh(TTVTTTTTTTTTAAGEST)R & 3 & & 2 & ce & 0.4 & 3 & +++ & $X$ & & & \\
\hline $186-207$ & $\begin{array}{c}\text { GVIGggghhh(TTVTTTTTTTTAAGEST) } \\
\text { R }\end{array}$ & 3 & & 3 & ce & $\begin{array}{ll}- \\
0.7\end{array}$ & 3 & + & $X$ & & & \\
\hline $186-207$ & GVIGggggh(TTVTTTTTTTTAAGEST)R & 4 & & 1 & ce & 0.8 & 3 & +++ & $X$ & & & \\
\hline $186-207$ & $\begin{array}{c}\text { GVIGgggghh(TTVTTTTTTTTAAGEST) } \\
\text { R }\end{array}$ & 4 & & 2 & ce & 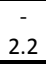 & 3 & ++ & $X$ & & & \\
\hline $186-207$ & $\begin{array}{c}\text { GVIGgggggh(TTVTTTTTTTTTAAGEST) } \\
\text { R }\end{array}$ & 5 & & 1 & ce & 0.3 & 3 & + & $X$ & & & \\
\hline $246-259$ & QIGCLAVgSQAGAMR & 1 & & & gS253e & $\begin{array}{l}- \\
4.1\end{array}$ & 2 & + & $X$ & & & \\
\hline
\end{tabular}




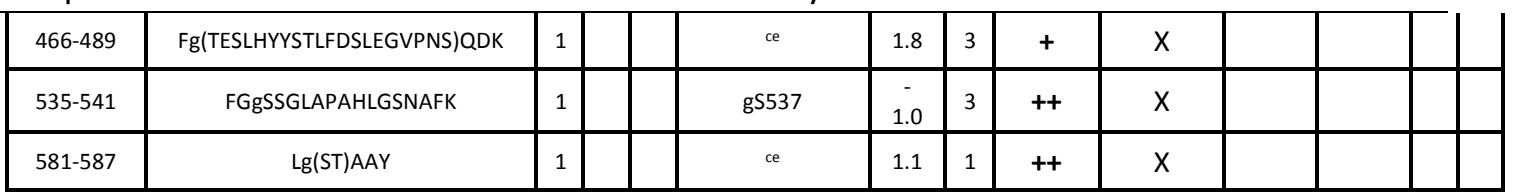

Table 2.4 - A summary of the number of modified residues and site-locations of O-GICNAc, phosphate, and/or Ohexose PTMs on RGA peptides from (a) RGA(+SEC). aPTM levels are reported as $\%$ total peptide abundance detected $,(+++++)=50+\%(++++)=20-49 \%,(+++)=10-19 \%,(++)=1-9 \%,(+)=<1 \%,(-)$ no modified peptide detected. \% Total abundances were calculated from ion currents observed in the main beam mass spectra [(modified peptide ion current)/(modified peptide + unmodified peptide ion current) x (100)]. ${ }^{b}$ Modified peptide containing this PTM site(s) was assigned by HPLC retention time and correct accurate mass, only. CPTM site is located on $S / T / Y\left(Y\right.$ - phosphorylation only) within (). 'AA mix of PTM sites were detected within brackets []. ${ }^{\text {eSite }}$ was determined by correct elution profile, accurate mass, and signature CAD fragmentation. ${ }^{f}$ Mass accuracy errors are reported in parts per million (PPM) relative to the mean experimental accurate mass of Angiotensin and Vasoactive Intestinal Peptide standard peptides. All cysteine residues were detected as carbamidomethylated. The digest(s) used to generate each peptide is denoted " $X$ " and the positive charge state ( $z$ ) reported. Peptides enriched using IMAC or derivatized with histamine are also denoted " $X$ ". O-GlcNAcylation, phosphorylation, and $O$-hexosylation are abbreviated by " $g$ ", " $p$ ", and " $h$ ", respectively.

Two of the localized phosphorylation sites, S23 and S170, matched motifs Ser-X-X-Ser/Thr

and/or Ser/Thr-X-X-X-Ser of Casein kinase 1 (CK1), a ubiquitous kinase in eukaryotes involved in signal transduction. No distinct consensus site exists for O-GIcNAc, but it is often located in Ser/Thr-rich regions of proteins ${ }^{11}$. This is the case for RGA as all four poly-Ser/Thr regions contain at least one O-GIcNAc. Peptides DHHQFQGRLSNHGTSSSSSSISK, SCSSPDSMVTSTSTGTQIGK, and GVIGTTVTTTTTTTTAAGESTR, representing three of these four regions, are all heavily modified with multiple combinations of PTMs. Peptides containing multiple Ser and Thr residues, especially in long stretches, present a significant analytical challenge because fragmentation must be nearly perfect to determine the exact site of modification, and these areas don't contribute any charge to help facilitate the ETD fragmentation that is needed. This problem is compounded by the fact that peptides modified on different sites but with the same number and types of PTMs (e.g. mono O-GlcNAcylation of S18 vs. mono O-GIcNAcylation of S20 for peptide DHHQFQGRLSNHGTSSSSSSISK) often coelute. Therefore, when the intact peptides are isolated in preparation for $\mathrm{MS}^{2}$, both forms are isolated and fragmented together, generating mixed ETD spectra as the ETD spectrum of mono O- 
series fragments contain an addition of 203 Da starting with $\mathrm{z}_{7}$ because one peptide form

contains an O-GIcNAc at S20 (Figure 2.19c). However, there are also $z_{7}$ and $z_{8}$ fragments that do not contain the +203 Da addition because another form of the peptide contains the O-GIcNAc on S18. The same phenomenon occurs with the $\mathrm{c}$ ion series as one sequences from the $\mathrm{N}$ terminus to the $\mathrm{C}$-terminus. Due to the strong abundance of these two sites and the ability of AspN to generate the modified peptide that also contains several basic His/Arg/Lys residues (predominantly $z=5$ ), these sites were localized by ETD despite the mixed spectrum. Still, it is important to consider that alternative mono O-GIcNAcylated forms that are present at much lower levels are very difficult to detect and validate, so there is a possibility that these exist too.

Several tools were implemented to aid in the detection and sequencing of these three PTMs. Immobilized Metal Affinity Chromatography was used to enrichment for phosphorylated peptides, an alternative site of cleavage (C-terminus of Met) was exploited using cyanogen bromide $(\mathrm{CNBr})$ to generate select peptides more amenable to ETD, and derivatization of carboxylic acids with histamine was used to enhance the charge density and overall charge states of certain peptides, also for the purpose of improving ETD efficiency.

\section{Enrichment of Phosphorylated Peptides by IMAC}

Due to the detection of RGA phosphorylation by tobacco kinases, enrichment by IMAC was employed to further improve the detection and localization of these sites. This takes advantage of the ability of Fe (III) to bind phosphate and later release it when it is reduced to Fe (II) (Figure 2.30). To prevent carboxylic acids from also binding to Fe (III), peptides were first esterified with methanolic $\mathrm{HCl}$ then loaded onto an IMAC column constructed in-house as described in Methods. Phosphorylated peptides were bound, extraneous peptides washed 
through, and after connecting the IMAC column to a C18 pre-column, Fe (III) was reduced to Fe

(II) with ascorbic acid to elute the enriched phosphorylated peptides onto the pre-column.

By enriching several different phosphorylated forms of DHHQFQGRLSNHGTSSSSSSISK, thereby

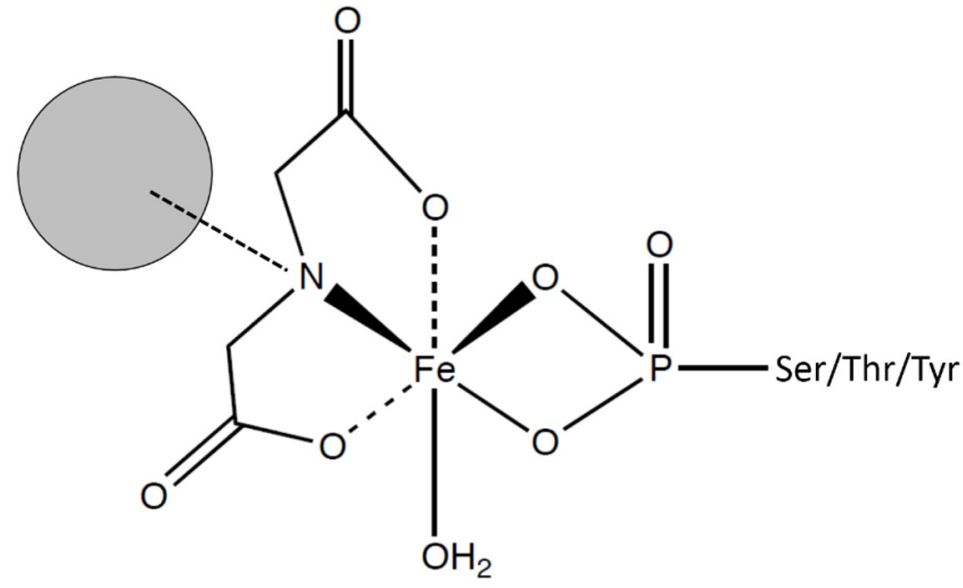

Figure $2.30-\mathrm{Fe}$ (III) coordinated to imidodiacetate on a solid support (POROS ${ }^{\oplus}$ MC20) specifically binds phosphorylated peptides. Nonphosphorylated peptides are washed away and the enriched phosphorylated peptides are released by reducing $\mathrm{Fe}$ (III) to Fe (II) using ascorbic acid.

mitigating dynamic range limitations that decrease isolation and sequencing ability, modified sites were elucidated for multiple PTM forms that would otherwise have been very difficult to map.

An alternative peptide cleavage technique using $\mathrm{CNBr}$

Several PTMs were detected on tryptic or Asp-N-generated peptides that were difficult to localize primarily because of a lack of charge to facilitate ETD. These included peptides GVIGTTVTTTTTTTTAAGESTR, SSEMAEVALK, and LSTAAY. The first, encompassing the poly-Thr region, had its ion current split between two charge states: $z=2$ and $z=3$. The first two protonation sites occurred on the $\mathrm{N}$-terminal amine and the guanidinium group of arginine. The third region available for protonation was the series of amides along the peptide backbone itself. Despite half of GVIGTTVTTTTTTTTAAGESTR charging to $z=3$ upon ESI, ETD fragmentation 
remained poor. This was likely due to two main factors: (1) the lack of internal basic and other residues empirically shown to frequently be associated with regions of high ETD fragmentation, and (2) low charge density relative to mass (i.e. relatively high $\mathrm{m} / \mathrm{z}$ ). Li et al compared fragmentation efficiencies between different residues using K-means clustering applied to several thousand peptides generated from LysC, GluC, and trypsin protein digests ${ }^{79}$. Fragmentation involving Gly, Ile, and Val tended to be relatively low in frequency, those involving Ala, Ser, and Thr average, and those involving residues Glu, His, Asn, Gln, Arg, and Lys were relatively high. The former two groups constitute 20 of the 22 residues in this peptide. A separate study by Good et al investigated the relationship between total charge, total mass, and charge density on ETD fragmentation efficiency using several thousand yeast and Arabidopsis peptides $(z=3-5)$ generated from Lys-C or trypsin digests ${ }^{80}$. They found that for charges states $z=3-5$, ETD fragmentation efficiency was independent of the total peptide mass or number of charges (Figure 2.31). 

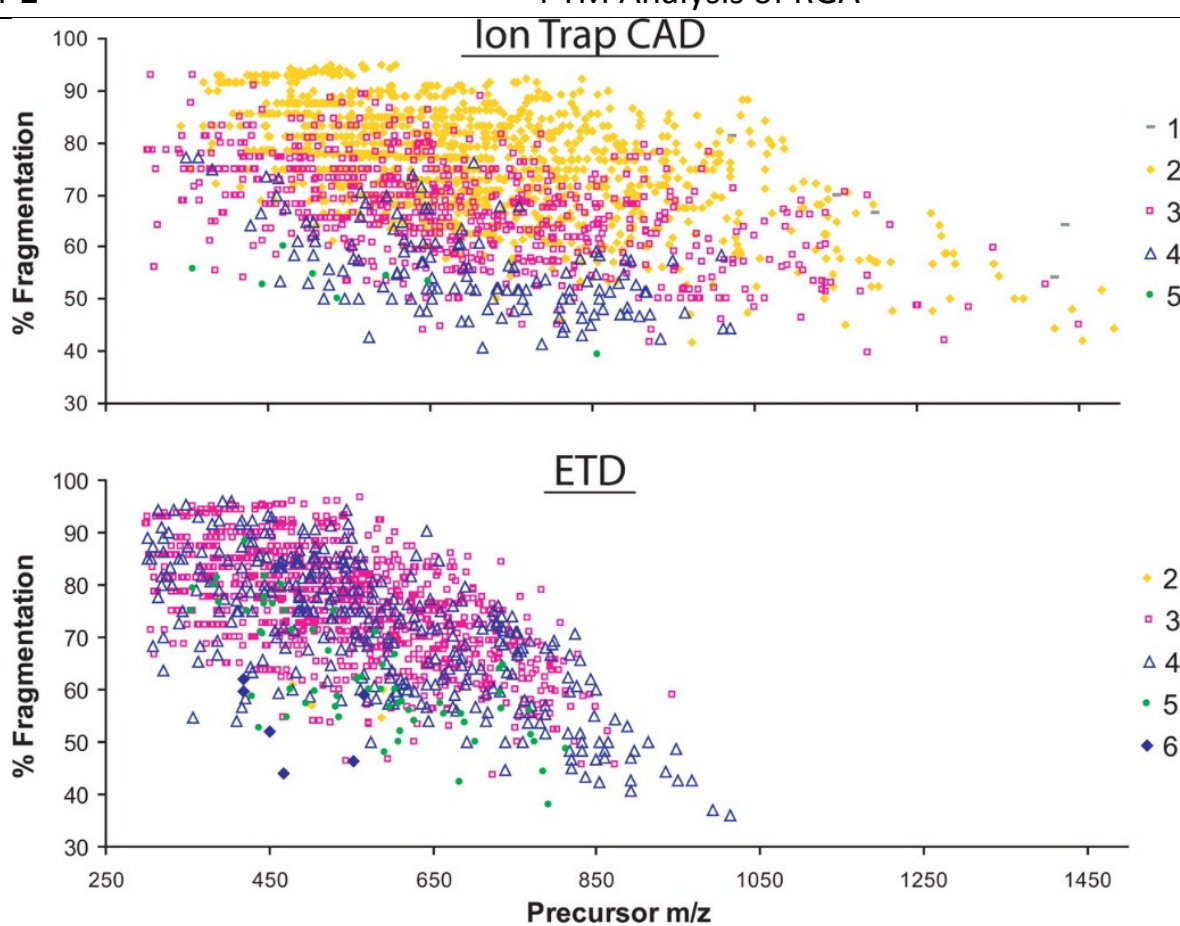

Figure 2.31 - ETD \% Fragmentation (defined by the number of observed fragment ions divided by the number of theoretical fragments) is relatively independent of charge state and peptide mass, but is highly dependent on charge density. Fragmentation efficiency decreases in a linear fashion as $\mathrm{m} / \mathrm{z}$ increases. Figure reproduced from ${ }^{80}$.

The key factor was charge density, with ETD fragmentation decreasing linearly with decreasing charge density. For $m / z>\sim 850$, or residue-to-charge ratios $>\sim 8$, ETD fragmentation efficiency suffered greatly with less than half of the theoretical fragment ions observed. The poly-Thr peptide and its PTM forms fit this challenging demographic as the $z=3$ form has 7.33 residues per charge and an unmodified mass-to-charge ratio of $710 \mathrm{~m} / \mathrm{z}$. The most abundant PTM forms, containing mono O-hexosylation and either di or tri O-GIcNAcylation, respectively hold mass-tocharge ratios of 899 and $967 \mathrm{~m} / z$. It is possible that the large sugar modifications themselves may contribute to diminished ETD efficiency by sterically hindering the interaction between the electron transfer reagent and carbonyl groups along the peptide backbone. Similarly, the large potential for hydrogen bonding may be realized in a way that partially folds and constricts this entire region of the peptide away from reagent. Also, the amide moieties on each O-GIcNAc are 
exact mimics of the electrophilic amides along the peptide backbone, and can contribute to "wasted" electron transfers that do not contribute to backbone fragmentation that generates c and $\mathrm{z}$ ions.

Although other enzymes exist in the proteomics repertoire to generate alternative peptides in these regions, none were suitable to generate peptides that would exhibit appropriate chromatographic elution and ETD fragmentation. One strategy used to address this challenge was to take advantage of a non-enzymatic cleavage method first described by Gross Witkop ${ }^{81}$ in which $\mathrm{CNBr}$ cleaves proteins C-terminally to Met residues converting them into homoserine lactones (Figure 2.32).

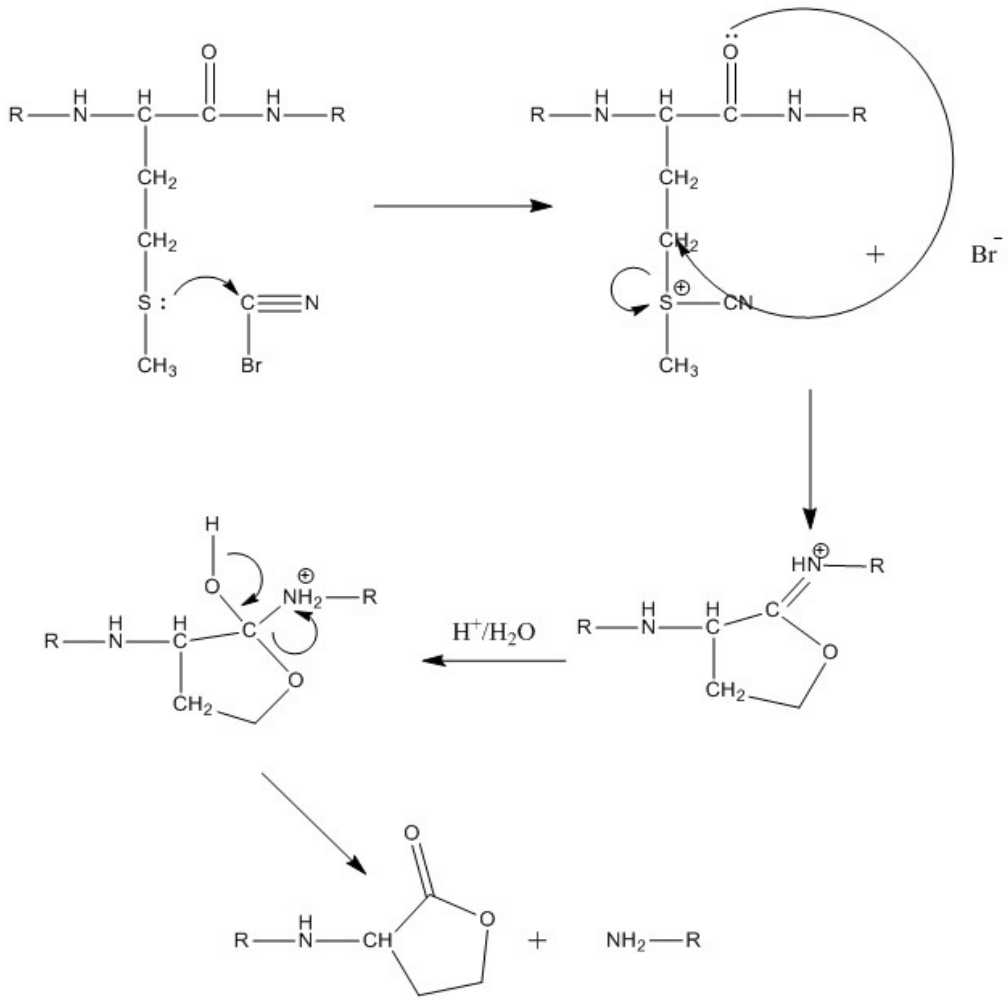

Figure 2.32 - Reaction scheme for cleavage of Met-X bonds by CNBr where $X$ is any amino acid. 
Because Met is a lower frequency amino acid in most proteins, cleavage by $\mathrm{CNBr}$ can be used to cleave proteins into a few large fragments very efficiently, as the mechanism does not require that a large enzyme access the sites to be cleaved. This may help further protein denaturation in preparation for efficient enzymatic digestion. Here, cleavage of Met's C-terminus was used to increase the library of peptides that would encompass the PTMs already detected, but also bear additional charge to enhance ETD fragmentation. RGA contains 19 Met residues and in silico cleavage by CNBr is reported in (Table 2.5) with peptides containing known modified regions highlighted in gray.

\begin{tabular}{|c|c|l|c|c|c|}
\hline Mass & Position & \multicolumn{1}{|c|}{ Peptide } & \# HKR & \# ST & Comments \\
\hline 3093.5 & $2-29$ & KRDHHQFQGRLSNHGTSSSSSSISKDKM & 8 & 9 & + \\
\hline 1114.5 & $32-42$ & VKKEEDGGGNM & 2 & 0 & + \\
\hline 1876 & $43-59$ & DDELLAVLGYKVRSSEM & 2 & 2 & + \\
\hline 1425.8 & $60-72$ & AEVALKLEQLETM & 1 & 1 & + \\
\hline 3386.5 & $74-103$ & SNVQEDGLSHLATDTVHYNPSELYSWLDNM & 2 & 6 & + \\
\hline 7424.6 & $104-174$ & $\begin{array}{l}\text { NSELNPPPLPASSNGLDPVLPSPEICGFPASDYDLKVIPG } \\
\text { NAIYQFPAIDSSSSSNNQNKRLKSCSSPDSM }\end{array}$ & 4 & 14 & - \\
\hline 5171.7 & $175-226$ & $\begin{array}{l}\text { VTSTSTGTQIGK*GVIGTTVTTTTTTTTAAGESTRSVILV } \\
\text { DS QENGVRLVHALM }\end{array}$ & $4 *$ & 20 & $?$ \\
\hline 3180.6 & $227-258$ & ACAEAIQQNNLTLAEALVKQIGCLAVSQAGAM & 1 & 2 & + \\
\hline 4056.1 & $259-293$ & RKVATYFAEALARRIYRLSPPQNQIDHCLSDTLQM & 4 & 6 & + \\
\hline 4339.2 & $294-330$ & HFYETCPYLKFAHFTANQAILEAFEGKKRVHVIDFSM & 7 & 3 & + \\
\hline 1108.6 & $331-340$ & NQGLQWPALM & 0 & 0 & + \\
\hline 6763.4 & $341-404$ & $\begin{array}{l}\text { QALALREGGPPTFRLTGIGPPAPDNSDHLHEVGCKLA } \\
\text { QLA EAIHVEFEYRGFVANSLADLDASM }\end{array}$ & 7 & 5 & $?$ \\
\hline
\end{tabular}




\begin{tabular}{|c|c|l|c|c|c|}
\hline 9657.1 & $405-491$ & $\begin{array}{l}\text { LELRPSTEAVAVNSVFELHKLLGRPGGIEKVLGVVKQI } \\
\text { K } \\
\text { PVIFTVVEQESNHNGPVFLDRFTESLHYYSTLFDSLEGV } \\
\text { P NSQDKVM }\end{array}$ & 11 & 10 & - \\
\hline 5796.8 & $492-545$ & $\begin{array}{l}\text { SEVYLGKQICNLVACEGPDRVERHETLSQWGNRFGSS } \\
\text { GLA PAHLGSNAFKQASM }\end{array}$ & 7 & 7 & $?$ \\
\hline 2254.1 & $546-566$ & LLSVFNSGQGYRVEESNGCLM & 1 & 3 & + \\
\hline 2372.3 & $567-587$ & LGWHTRPLITTSAWKLSTAAY & 3 & 6 & + \\
\hline
\end{tabular}

Table 2.5 - Theoretical peptides generated by in silico digest of RGA by CNBr. Peptide masses (Da) and position numbers are listed along with the number of basic (\# HKR) and GlcNAc-modifiable (\# ST) residues for each theoretical peptide. The comments section refers to peptides predicted to be detected (+) or not detected (-), with (?) indicating uncertainty of detection. *Indicates lysine insertion.

$\mathrm{CNBr}$ cleavage theoretically generates yet another form of two adjacent and heavily modified poly-Ser/Thr regions, consisting of residues T190-T206. This peptide, VTSTSTGTQIGKGVIGTTVTTTTTTTTAAGESTRSVILVDSQENGVRLVHALm where lowercase “ $\mathrm{m}$ ” represents the newly formed homoserine lactone residue, would contain three more basic residues (including the lysine insertion) than its tryptic counterpart. In addition, it would contain three carboxylic acids able to be derivatized with histamine to carry charge. Then, if all basic functional groups plus the peptide backbone were protonated upon $\mathrm{ESI}$, this $\mathrm{CNBr}$ generated peptide would contain 9 charges for a residue-to-charge ratio of 5.8. Non-PTM forms of the $z=9$ peptide would hold a mass-to-charge ratio of $611 \mathrm{~m} / z$.

To test Met cleavage by $\mathrm{CNBr}$, a slightly modified method from Yaroslav et al. ${ }^{82}$ was used to cleave the single Met residue on human recombinant protein $\mathrm{H} 4$ and generate extreme C-terminal peptide, DVVYALKRQGRTLYGFGG. Dissolving CNBr in acetonitrile and adding a 20100 fold excess over Met residues to the sample dissolved in 70\% tri-flouroacetic acid (TFA), as described in the literature, failed to generate the target peptide. Instead, as described in Materials and Methods, a small crystal ( $<1 \mathrm{mg}$ ) of $\mathrm{CNBr}$ was added directly to the sample. Mass spectrometric analysis revealed dominant signal from the $\mathrm{CNBr}$-derived peptide, 
DVVYALKRQGRTLYGFGG, as theoretically predicted. The enhancement of cleavage was probably due to the very large excess of $\mathrm{CNBr}$ over Met (>1000x) and potentially increased water access to Met, because no organic solvent was used.

Next, the method was applied to whole RGA-G185_G186insK, eluted from anti-FLAG beads after immunoprecipitation with acetic acid. The elution was performed to avoid potential degradation of the agarose beads by strong acid which could unnecessarily complicate the sample. Unfortunately, the theoretical poly-Ser/Thr peptide was not detected, apparently being too lengthy to efficiently elute from the chromatographic columns. However, in silico $\mathrm{CNBr}$ subcleavage of an AspN digest revealed three unique peptides (one synonymous with $\mathrm{CNBr}$-only cleavage) that would contain previously detected PTMs. These peptides are highlighted in gray in Table 2.6.

\begin{tabular}{|r|c|l|c|c|c|}
\hline \multicolumn{1}{|r|}{ Mass } & Position & & $\begin{array}{c}\# \\
\text { HKR }\end{array}$ & $\begin{array}{c}\# \\
\text { ST }\end{array}$ & Comments \\
\hline 344.2 & $27-29$ & DKM & 1 & 0 & - \\
\hline 631.4 & $32-36$ & VKKEE & 2 & 0 & - \\
\hline 1760.9 & $44-59$ & DELLAVLGYKVRSSEM & 2 & 2 & + \\
\hline 1425.8 & $60-72$ & AEVALKLEQLETM & 1 & 0 & + \\
\hline 575.3 & $74-78$ & SNVQE & 0 & 1 & $?$ \\
\hline 330.1 & $101-103$ & DNM & 0 & 0 & - \\
\hline 1604.8 & $104-119$ & LSELNPPPLPASSNGL & 0 & 3 & + \\
\hline 1519.8 & $213-226$ & DSQENGVRLVHALM & 2 & 1 & - \\
\hline 3180.6 & $227-258$ & ACAEAIQQNNLTLAEALVKQIGCLAVSQAGAM & 1 & 2 & - \\
\hline 2960.6 & $259-283$ & RKVATYFAEALARRIYRLSPPQNQI & 5 & 2 & - \\
\hline 558.3 & $289-293$ & DTLQM & 0 & 1 & $?$ \\
\hline 3907.0 & $294-326$ & HFYETCPYLKFAHFTANQAILEAFEGKKRVHVI & 7 & 2 & + \\
\hline 450.2 & $327-330$ & DFSM & 0 & 1 & - \\
\hline 1108.6 & $330-339$ & NQGLQWPALM & 0 & 0 & + \\
\hline 2315.3 & $340-363$ & QALALREGGPPTFRLTGIGPPAP & 2 & 2 & + \\
\hline 374.1 & $401-404$ & DASM & 0 & 1 & - \\
\hline 713.4 & $405-410$ & LELRPS & 1 & 1 & $?$ \\
\hline
\end{tabular}




\begin{tabular}{|r|l|l|c|c|c|}
\hline 443.2 & $488-491$ & DKVM & 1 & 0 & - \\
\hline 1921.9 & $492-509$ & SEVYLGKQICNLVACEGP & 1 & 1 & + \\
\hline 3892.9 & $510-545$ & $\begin{array}{l}\text { DRVERHETLSQWGNRFGSSGLAPAHLGSNAFKQ } \\
\text { ASM }\end{array}$ & 6 & 6 & + \\
\hline 2254.1 & $546-566$ & LLSVFNSGQGYRVEESNGCLM & 1 & 3 & + \\
\hline 2372.3 & $567-587$ & LGWHTRPLITTSAWKLSTAAY & 3 & 6 & + \\
\hline
\end{tabular}

Table 2.6 - Theoretical peptides generated by in silico digest of RGA by AspN + CNBr. Peptide masses (Da) and position numbers are listed along with the number of basic (\# HKR) and GICNAc-modifiable (\# ST) residues for each theoretical peptide. The comments section refers to peptides predicted to be detected (+) or not detected (-), with (?) indicating uncertainty of detection.

Peptides DELLAVLGYKVRSSEm and LGWHTRPLITTSAWKLSTAAY contained two and three more basic residues respectively, than their tryptic counterparts, SSEMAVALK and LSTAAY. Peptide LSELNPPPLPASSNGL was a slightly more compact version of its AspN counterpart, having lost 3 residues from its $\mathrm{N}$-terminus. While this improved the overall charge state potential for two out of these three peptides, all three still lacked a basic residue on the C-terminal side of the Ser/Thr residues in question. Electron transfer to the carbonyl group consumes one positive charge from the peptide precursor and a reaction cascade proceeds to break one amide bond per molecule, generating one $\mathrm{c}$ and $\mathrm{z} \cdot$ ion each. If there is not at least one charge remaining on each fragment ion, it will not be detected by the mass spectrometer. Therefore for all three<smiles>[R]C([NH3+])C(=N)O[TlH]</smiles>

No charge

$z \cdot$ ion can not be detected

Figure $2.33-E T D$ consumes one charge, requiring a minimum of 3 charges for $c$ and $z \cdot$ ions to both be detected. If no protonation exists C-terminally to the cleavage site, the $z$ ion will not be detected. 
peptides above, it would be impossible to detect any z ions within the Ser/Thr regions (Figure

\subsection{3).}

Loss of one half of the ions in this region severely hinders the ability to confidently assign the PTM site(s) and often precludes the ability to do so completely. Therefore, $\mathrm{CNBr}$ subdigestion was combined with chemical derivatization to add C-terminal charges to these modified peptides before ETD analysis.

Enhancement of ETD by Derivatization of Carboxylic Acids with Histamine

As previously described in this section, one technique that may be used to add charge is the derivatization of carboxylic acids with histamine ${ }^{45}$. The basic imidazole ring is a strong candidate for protonation, adding charge to the three types of acidic residues: Asp, Glu, and Ctermini. Therefore, this method is useful for simultaneously adding charge throughout peptides in addition to adding C-terminal charge. Derivatization reactions were performed on Asp-N generated peptides sub-cleaved with $\mathrm{CNBr}$ in the same manner as previously described in this chapter. Tryptic RGA peptides were also derivatized with histamine, especially in the attempt to improve ETD fragmentation of poly-Ser/Thr peptides VIPGNAIYQFPAIDSSSSSNNQNKR and GVIGTTVTTTTTTTTAAGESTR, neither of whose PTMs could be site-mapped.

Following derivatization of Asp and the C-terminus with histamine, VIPGNAIYQFPAIDSSSSSNNQNKR was charge enhanced from its previous $z=3$ to $z=4$ or 5 . Figure 2.34 illustrates the improvement in ETD of VIPGNAIYQFPAIDSSSSSNNQNKR before and after full derivatization with histamine. 


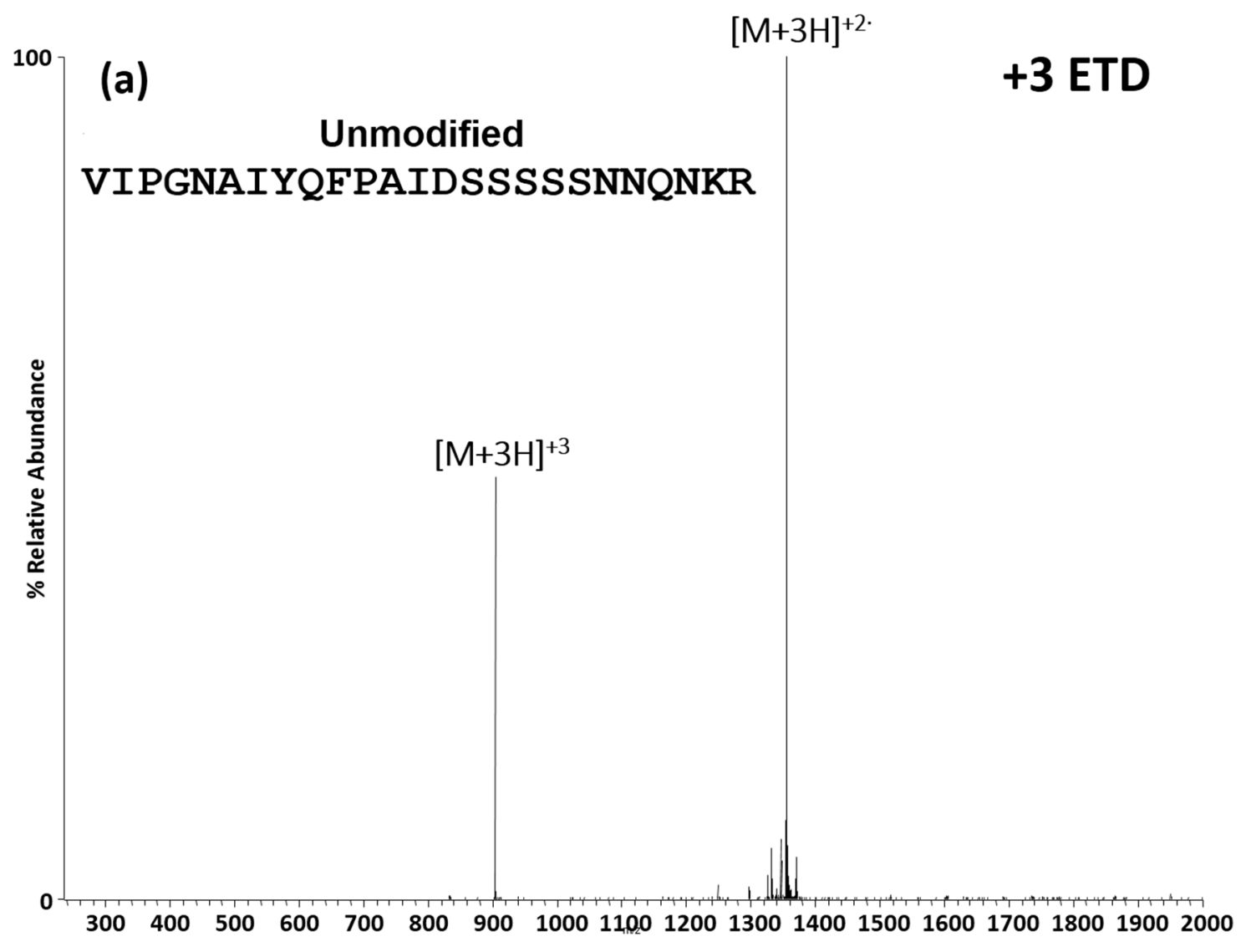




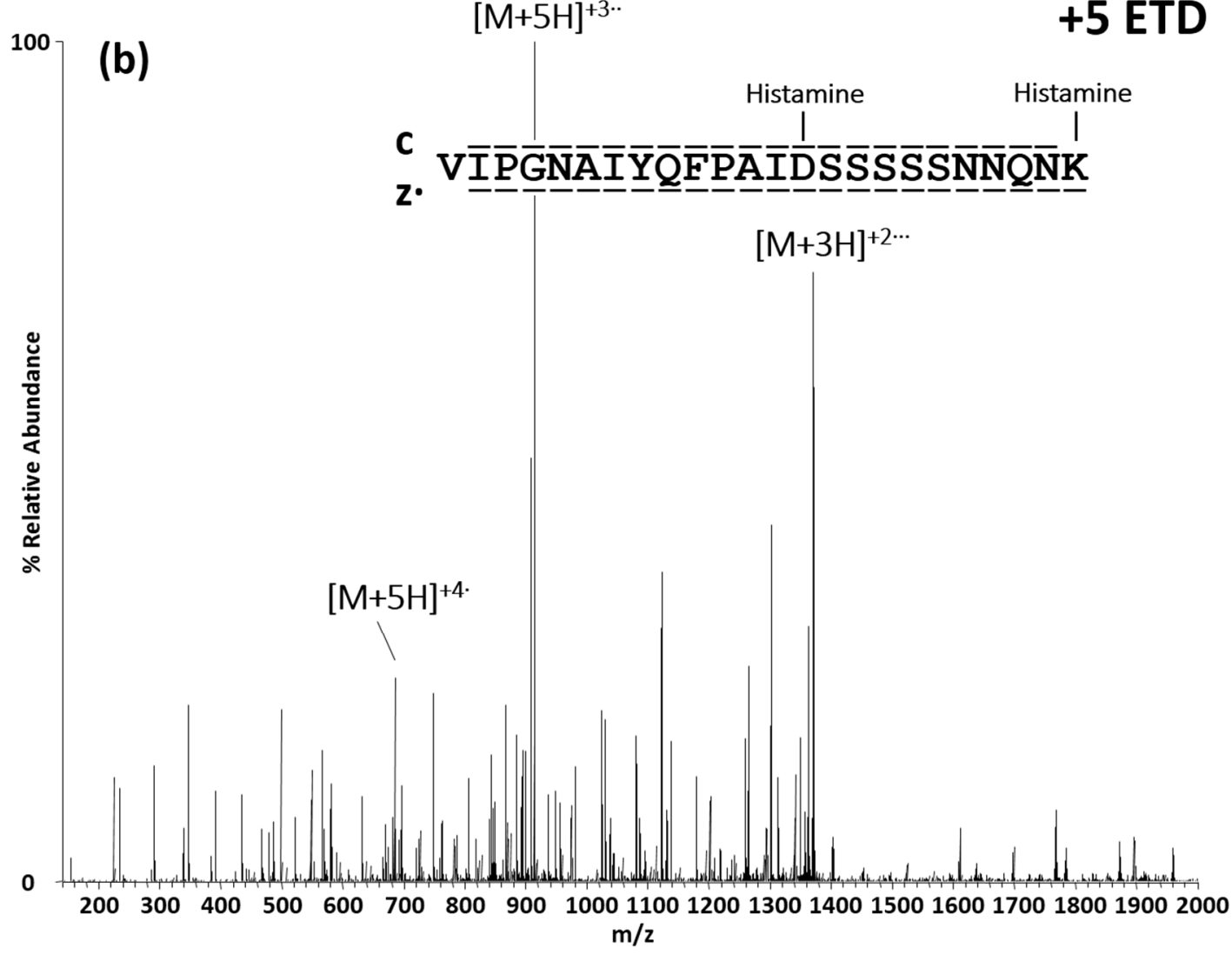

Figure 2.34 - Derivatization of Asp and the C-terminus of unmodified VIPGNAIYQFPAIDSSSSSNNQNK with histamine increased the charge state from $z=3(a)$ to $z=5(b)$ and improved sequence coverage from nil to full. Black bars represent observed $c$ and $z$. fragment ions.

Unfortunately, the ion current of the fully derivatized, $z=5$ peptide only accounted for $\sim 10 \%$ of its total ion current detected. This was due to incomplete reaction efficiency, side reaction of Asp forming a succinimide (Figure 2.35), and ion current splitting evenly between charge states +4 and +5 . These ion current divisions combined with the already low abundance of both $0-$ GIcNAc and phosphate PTMs still prevented even the $z=5$ PTM forms from fragmenting well by ETD. Because GVIGTTVTTTTTTTTTAAGESTR similarly contained two derivitazable sites, Glu and the C-terminus, it was also charge enhanced from its predominant $z=3$ to $z=4$ or 5 as well. Fortunately, its PTM forms were more abundant than its unmodified form although the ion current was still split amongst 26 distinct combinations of PTMs, plus additional isobaric 
combinations having the same $\mathrm{m} / \mathrm{z}$ but different primary locations. The ion current was again additionally evenly divided between $z=4$ and 5 following ESI. Despite this, tens of fmols of several PTM forms were available for ETD, but it did not improve substantially, and the sites remained virtually unmappable. The charge likely remained too localized near the peptides' Cterminus, as opposed to the $\mathrm{N}$-terminus and center where the majority of modifiable residues were located.

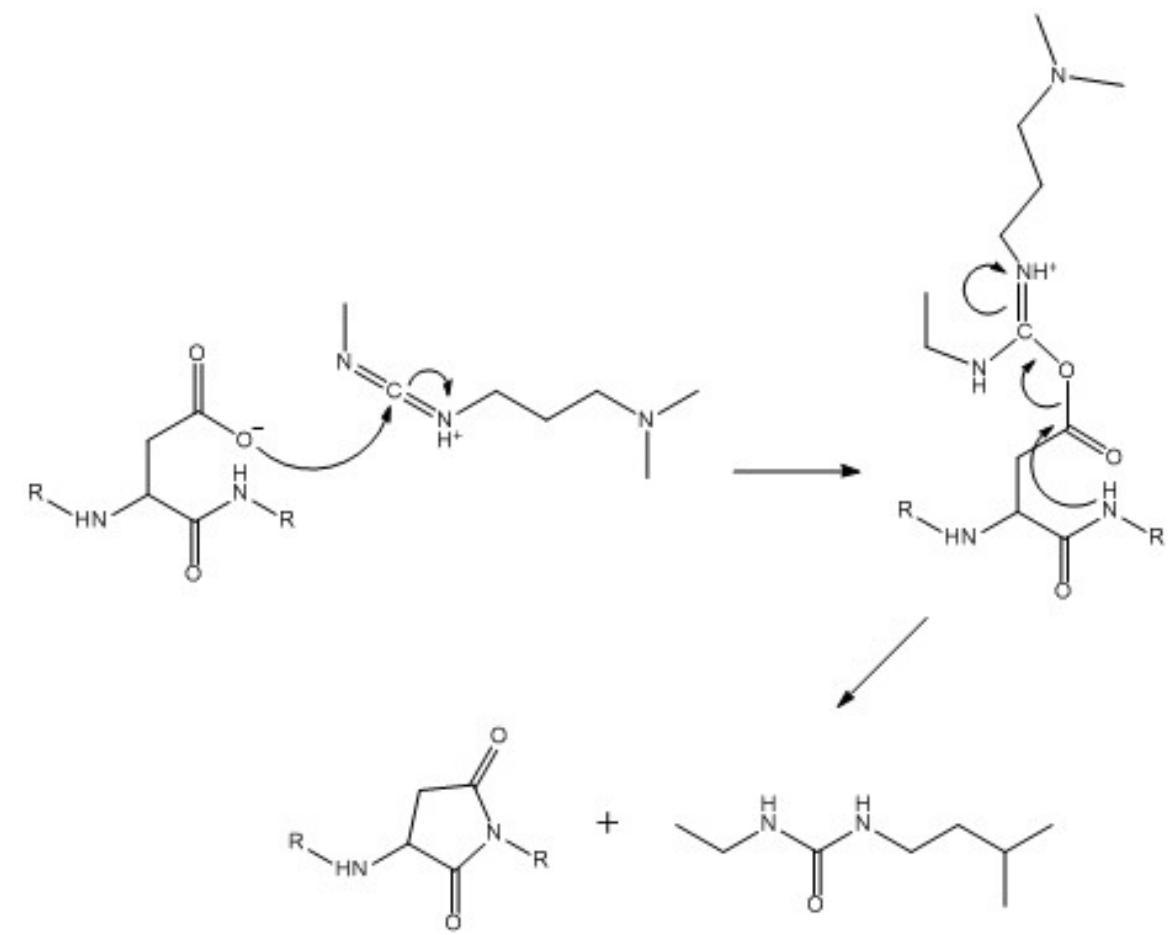

Figure 2.35 - Reaction scheme of Asp residues forming stable succinimide side-products.

Histamine derivatization was more successful in improving ETD fragmentation for the peptides cleaved by Asp-N and CNBr. In the case of DELLAVLGYKVRSSEm, which lacks the Cterminal carboxylic acid due to homoserine lactone formation, a carboxylic acid still exists Cterminally to the tandem Ser residues because of the adjacent Glu residue. Asp-N peptide DNMLSELNPPPLPASSNGL was subcleaved with CNBr which had two advantages. The first advantage was to avoid potentially splitting ion current at a site where charge enhancement 
was not critical. Aspartic acids have a propensity to form backbone succinimides upon

activation by EDC. A neighboring amide may attack the carbonyl carbon of Asp's carboxylic acid, forming the succinimide which is stable at the acidic $\mathrm{pH}(5.5)$ used for derivatization ${ }^{45,83}$. The CNBr-subcleaved peptide, LSELNPPPLPASSNGL, contained an N-terminal primary amine two carboxylic acids, Glu and the C-terminus, with the potential to hold three charges following histamine derivatization. A second advantage was to gain a higher charge density to improve ETD. Derivatization of all three CNBr-subcleaved AspN peptides enabled the successful ETD sequencing of their unmodified forms. Peptide LGWHTRPLITTSAWKLSTAAY was enhanced from $z=5$ to $z=6$ with a new charge on the C-terminus, but the low abundance $(<1 \%)$ of the 0 GlcNAcylated form still prevented the generation of a quality O-GlcNAcylated ETD spectrum. Despite this, DaELLAVLGYKVRSSaEm $(z=4)$, where "a" precedes residues derivatized with histamine, could now be sequenced by ETD to reveal S56 as the major O-GIcNAcylation and Ohexosylation site in this region with O-GlcNAc being more abundant than O-hexose. A more minor site, S57 also separately contained both PTMs, with O-GIcNAc also in higher abundance than O-hexose. Finally, LSaELNPPPLPASSNGaL $(z=3)$ was now sufficiently charge-enhanced to allow for unambiguous sequencing of four of its five PTM forms (Figure 2.36). 

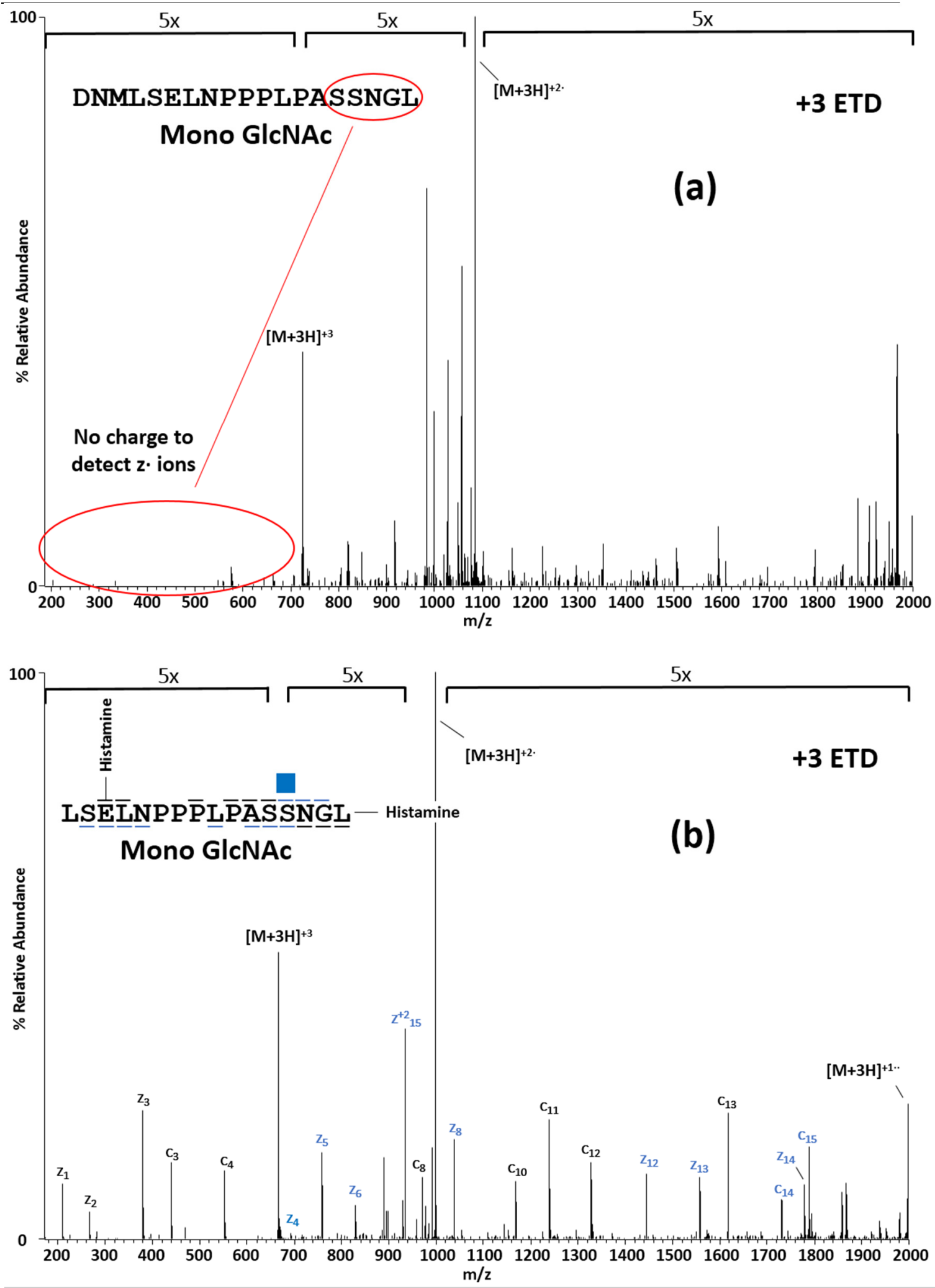


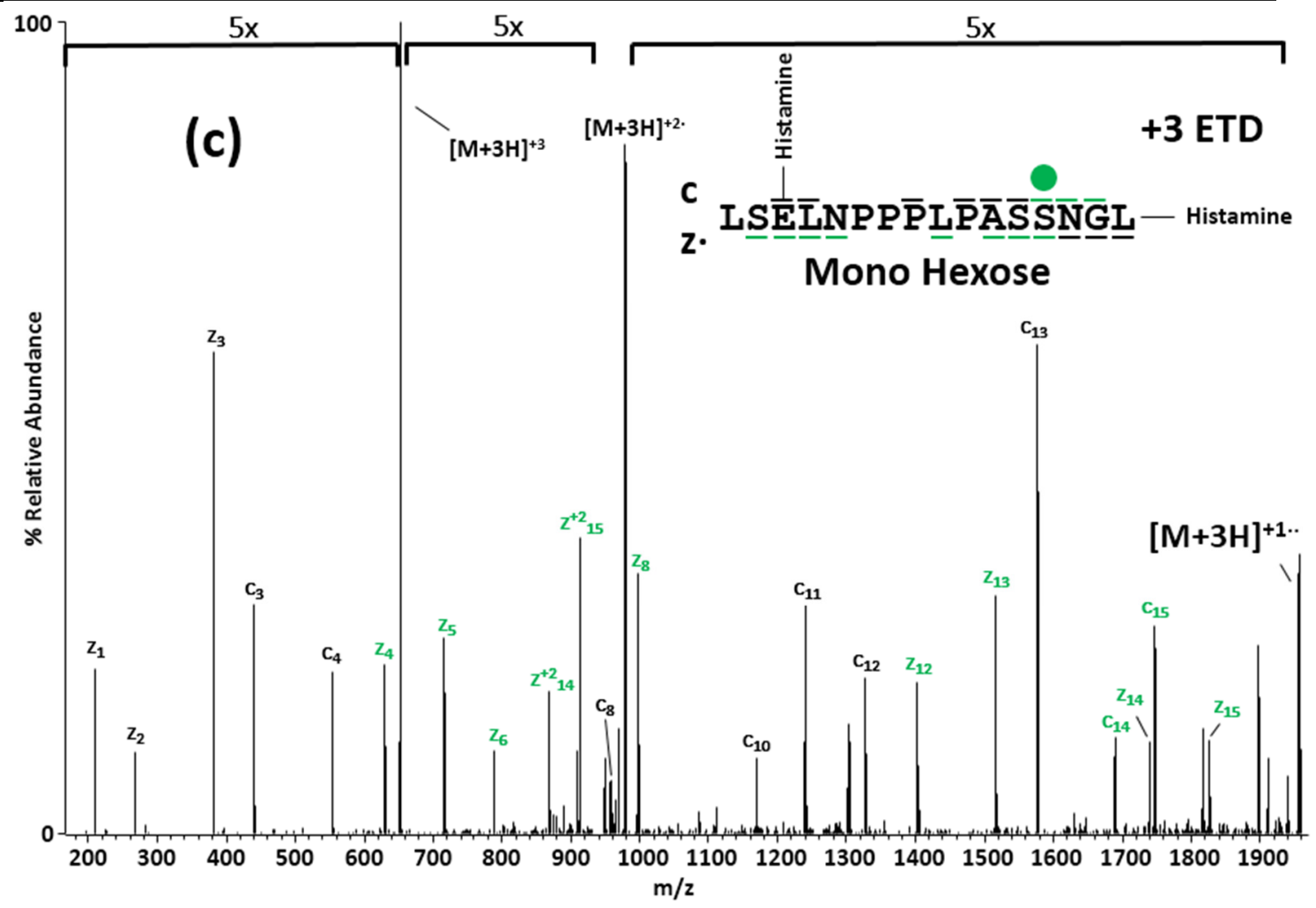

Figure 2.36 -DNMLSELNPPPLPASSNGL does not efficiently fragment by ETD, and no charge exists on the Cterminus to detect low mass $z$ ions (a) and confidently locate PTMs. Charge density was increased by subcleaving with $\mathrm{CNBr}$ and derivatizing carboxylic acids with histamine to place the charge at strategic locations. Mono-OGIcNAcylated (b) and O-hexosylated (c) ETD spectra of derivatized peptide LSELNPPPLPASSNGL allows unambiguous localization of each PTM.

Mono-O-GlcNAcylation and O-hexosylation were detected on residue S116.

Interestingly, both PTMs were also detected simultaneously in two forms: O-hexose on S115 and O-GIcNAc on S116 was the major form and vice versa was the minor form. Subcleavage of Asp-N-digested peptides with CNBR, combined with derivatization by histamine, enabled the sequencing of 9 distinct RGA PTM peptides forms.

Analysis of RGA(+SEC) PTMs

Overall, the RGA(+SEC) tobacco system produced highly modified RGA protein with respect to the number of sites modified, the number of PTM combinations observed, and the abundance of these PTMs relative to the total abundance of the peptide detected. A total of 13 
peptides were detected containing O-GIcNAcylation, phosphorylation, and/or O-hexosylation.

These 13 peptides contained a combined total of 89 distinct PTM forms, with the majority due to 3 of the 4 poly-Ser/Thr peptides bearing 30, 12, and 26 PTM forms, respectively (Table 2.7).

\begin{tabular}{|c|c|c|c|c|c|c|c|c|}
\hline \multirow{3}{*}{ Peptide } & \multicolumn{8}{|c|}{ Forms containing: } \\
\hline & \multicolumn{2}{|c|}{ O-GIcNAc } & \multicolumn{2}{|c|}{ Phosphate } & \multicolumn{2}{|c|}{ O-Hexose } & \multicolumn{2}{|c|}{ Total } \\
\hline & 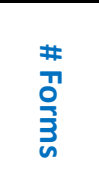 & 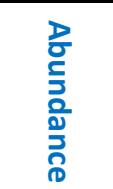 & 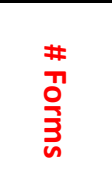 & 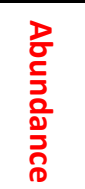 & 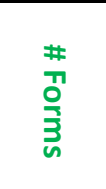 & 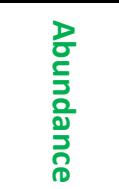 & $\begin{array}{l}\# \\
\text { \# } \\
\stackrel{3}{3} \\
3 \\
n\end{array}$ & $\begin{array}{l}\frac{D}{0} \\
\frac{5}{3} \\
\frac{2}{2} \\
\frac{2}{0}\end{array}$ \\
\hline $\begin{array}{l}\text { DHHQFQGRLSNHGTSSSSSSI } \\
\text { SK }\end{array}$ & 19 & +++++ & 16 & ++ & 11 & ++ & 30 & +++++ \\
\hline DELLAVLGYKVRSSEM & 3 & ++++ & & & 2 & ++ & 5 & ++++ \\
\hline DGLHLAT & 1 & ++ & & & 1 & + & 2 & ++ \\
\hline DTVHYNPSELYSWL & 1 & + & & & & & 1 & + \\
\hline DPVLPSPEICGFPAS & 1 & ++ & 1 & + & & & 2 & ++ \\
\hline LSELNPPPLPASSNGL & 4 & +++++ & & & 3 & ++++ & 5 & +++++ \\
\hline $\begin{array}{l}\text { VIPGNAIYQFPAIDSSSSSNNQ } \\
\text { NKR }\end{array}$ & 1 & ++ & 1 & + & & & 2 & ++ \\
\hline SCSSPDSMVTSTSTGTQIGK & 10 & +++++ & 4 & +++ & 2 & ++ & 12 & +++++ \\
\hline GVIGTTVTTTTTTTTTAAGESTR & 21 & +++++ & & & 20 & +++++ & 26 & +++++ \\
\hline QIGCLAVSQAGAMR & 1 & + & & & & & 1 & + \\
\hline $\begin{array}{l}\text { FTESLHYYSTLFDSLEGVPNSQ } \\
\text { DK }\end{array}$ & 1 & + & & & & & 1 & + \\
\hline FGSSGLAPAHLGSNAFK & 1 & ++ & & & & & 1 & ++ \\
\hline LSTAAY & 1 & ++ & & & & & 1 & ++ \\
\hline & Total & & Total & & Total & & Total & \\
\hline & 65 & & 22 & & 39 & & 89 & \\
\hline & $73 \%$ & & $25 \%$ & & $44 \%$ & & & \\
\hline
\end{tabular}

Table 2.7-Summary of the 13 modified RGA(+SEC) peptides that contain O-GlcNAc, phosphate, and/or O-hexose. The number of unique forms containing each PTM are reported as well as the total abundance of peptide containing each PTM. PTM levels are reported as \% total peptide abundance detected, $(+++++)=50+\%(++++)=20-49 \%,(+++)=10-$ $19 \%,(++)=1-9 \%,(+)=<1 \%$. \% Total abundances were calculated from ion currents observed in the main beam mass spectra [(modified peptide ion current)/(modified peptide + unmodified peptide ion current) x (100)]. 


\begin{tabular}{|c|c|c|c|c|c|c|c|c|}
\hline \multirow[b]{2}{*}{ Peptide } & \multicolumn{8}{|c|}{ Number of Forms containing: } \\
\hline & 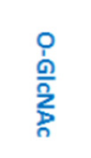 & 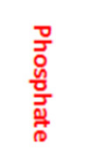 & 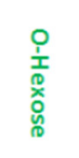 & 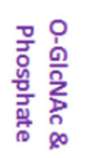 & 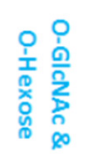 & 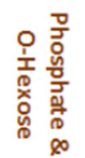 & 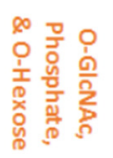 & $\begin{array}{l}\overrightarrow{1} \\
\underline{\underline{0}}\end{array}$ \\
\hline DHHQFQGRLSNHGTSSSSSSISK & 6 & 5 & 4 & 8 & 4 & 2 & 1 & 30 \\
\hline DELLAVLGYKVRSSEM & 3 & 0 & 2 & & & & & 5 \\
\hline DGLHLAT & 1 & 0 & 1 & & & & & 2 \\
\hline DTVHYNPSELYSWL & 1 & 0 & 0 & & & & & 1 \\
\hline DPVLPSPEICGFPAS & 1 & 1 & 0 & & & & & 2 \\
\hline LSELNPPPLPASSNGL & 2 & 0 & 1 & & 2 & & & 5 \\
\hline VIPGNAIYQFPAIDSSSSSNNQNKR & 1 & 1 & 0 & & & & & 2 \\
\hline SCSSPDSMVTSTSTGTQIGK & 6 & 1 & 1 & 3 & 1 & & & 12 \\
\hline GVIGTTVTIIIIITIAAGESTR & 6 & 0 & 5 & & 15 & & & 26 \\
\hline QIGCLAVSQAGAMR & 1 & 0 & 0 & & & & & 1 \\
\hline FTESLHYYSTLFDSLEGVPNSQDK & 1 & 0 & 0 & & & & & 1 \\
\hline FGSSGLAPAHLGSNAFK & 1 & 0 & 0 & & & & & 1 \\
\hline \multirow[t]{4}{*}{ LSTAAY } & 1 & 0 & 0 & & & & & 1 \\
\hline & Total & Total & Total & Total & Total & Total & Total & Total \\
\hline & 31 & 8 & 14 & 11 & 22 & 2 & 1 & 89 \\
\hline & $35 \%$ & $9 \%$ & $16 \%$ & $12 \%$ & $25 \%$ & $2 \%$ & $1 \%$ & \\
\hline
\end{tabular}

\begin{tabular}{|c|c|c|c|c|c|c|c|c|}
\hline \multirow[b]{2}{*}{ Peptide } & \multicolumn{8}{|c|}{ Abundance of Forms containing: } \\
\hline & 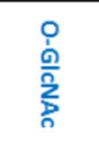 & 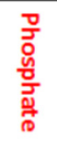 & 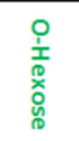 & 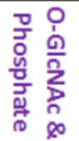 & 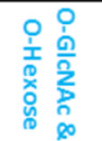 & 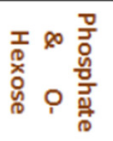 & 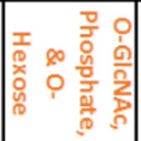 & $\begin{array}{l}\overrightarrow{1} \\
\text { 离 } \\
\underline{\underline{n}}\end{array}$ \\
\hline DHHQFQGRLSNHGTSSSSSSISK & $\mathrm{HH+H}$ & + & + & $H$ & + & + & + & $\mathrm{H+H}$ \\
\hline DELLAVLGYKVRSSEM & +++ & & + & & & & & $+H$ \\
\hline DGLHLAT & + & & + & & & & & + \\
\hline DTVHYNPSELYSWL & + & & & & & & & + \\
\hline DPVLPSPEICGFPAS & + & + & & & & & & + \\
\hline LSELNPPPLPASSNGL & $+H+1+$ & & $+H+$ & & + & & & ++++4 \\
\hline VIPGNAIYQFPAIDSSSSSNNQNKR & + & + & & & & & & + \\
\hline SCSSPDSMVTSTSTGTQIGK & $+H+$ & ++ & + & + & + & & & ++++ \\
\hline GVIGTTVTIIIIITAAGESTR & $\mathrm{H+H}$ & & + & & $\mathrm{HHH}$ & & & $\mathrm{H+H+H}$ \\
\hline QIGCLAVSQAGAMR & + & & & & & & & + \\
\hline FTESLHYYSTLFDSLEGVPNSQDK & + & & & & & & & + \\
\hline FGSSGLAPAHLGSNAFK & + & & & & & & & + \\
\hline LSTAAY & + & & & & & & & + \\
\hline
\end{tabular}

Table 2.8 - Summary of modified RGA(+SEC) peptides expanded to report the number and abundance of peptides containing O-GICNAC, phosphate, and O-hexose exclusively or in combination

Of the 89 different PTM forms from the 13 peptides containing PTMS, 31 contained only O-

GlcNAc, 8 only phosphate, 14 only O-hexose, 11 O-GIcNAc and phosphate, 22 O-GlcNAc and O-

hexose, 2 phosphate and O-hexose, and 1 O-GIcNAc, phosphate, and O-hexose (Table 2.8). 
O-GIcNAcylation was most prevalent, as all 13 peptides found to be post-translationally modified contained 1 or more O-GlcNAcylated forms, with 2 or more O-GIcNAcs detected simultaneously on 5 of them. Of these 5 peptides able to be multiply O-GlcNAcylated, 4 were $>50 \%$ modified with O-GlcNAcylation. Forms of these same 4 peptides were also detected containing 1 or more O-GlcNAcs simultaneously with phosphorylation and/or O-hexosylation. In all, 23 O-GIcNAcylation sites were detected on RGA(+SEC) and 14 were unambiguously localized. It is likely that additional sites exist on PTM peptides bearing isobaric $\mathrm{m} / \mathrm{z}$ values with those forms already sequenced, with the resulting mixed $\mathrm{MS}^{2}$ spectra preventing their identification. O-hexosylation was the second most prevalent PTM with 20-50\% of LSELNPPPLPASSNGL modified and $>50 \%$ of GVIGTTVTTTTTTTTAAGESTR modified. The majority of the former's Ohexosylation was detected apart from O-GIcNAc, although the two forms were also present simultaneously in lesser abundance. In the latter, O-hexosylation was also present but the vast majority was detected simultaneously with O-GIcNAcylation in 15 different PTM combinations. A total of 14 confirmed O-hexosylation sites were detected on RGA(+SEC) with 9 of these sitelocalized. Phosphorylation was the least abundant PTM, yet six sites were still detected on four peptides, and five of these could be site-localized. On two of these, SCSSPDSMVTSTSTGTQIGK and DHHQFQGRLSNHGTSSSSSSISK, phosphorylation was detected on forms bearing and lacking O-GlcNAcylation. Likewise, these two peptides also contained O-hexose forms bearing and lacking O-GIcNAcylation. One peptide, DHHQFQGRLSNHGTSSSSSSISK, was detected containing all PTMs both alone and with all possible PTM combinations, including O-GlcNAc, phosphorylation, and O-hexose all simultaneously present. Notably, it was the only peptide to contain all potential combinations and was the most diversely modified region with 30 total PTM forms. It was O-GlcNAcylated in 19 forms of these forms, phosphorylated in 16 forms, and O-hexosylated in 11 forms detected. This is significant, because it reveals that at least 30 
modified forms of RGA(+SEC) exist. In fact, >30 PTM forms of RGA were observed to exist because 3 other peptides were $>50 \%$ modified with multiple PTM combinations. Multiple PTM forms of domains S13-S25, S167-T181, and T190-T206 must coexist on the same protein molecules.

Site Locations of RGA PTMs

The vast majority of RGA PTMs occur on the N-terminal half of the protein. All polySer/Thr regions are contained in this region as well. Eight distinct regions of RGA were found to be modified by more than one type of PTM, and five of these regions contained multiple simultaneous PTMs of the same and/or different type.

The most diversely modified region is encompassed by residues S13-S25. The majority of this region is detected with an even mix of O-GlcNAcylation on S18 or S20. An abundant di-OGlcNAcylated form was detected bearing O-GlcNAcs detected most strongly on S18 and S19, with others co-eluting forms that contained O-GIcNAc(s) between T17-S22. One interpretation of this is that O-GIcNAcylation of S18 can direct O-GIcNAcylation of S19 or vice versa. This conclusion must be considered cautiously though, because mixed isobaric spectra limit one's ability to detect PTMs on residues located in between two others found to contain PTMs. They also hurt the limit of PTM detection. Therefore, it is possible that this region was also mono-OGlcNAcylated at S19 but wasn't detected because it would have been nearly impossible to distinguish whether the modified $\mathrm{c}_{16}$ and $\mathrm{z}_{8}$ fragments ions were a result of the addition of $\mathrm{O}$ GIcNAc on S19, or the mix on S18 and S20, respectively (Figure 2.19b\&c). The data indicates additional sites were modified in the di-O-GlcNAcylated form, implying that the first O-GIcNAc may help facilitate recruitment of OGT and/or exert control of where it attaches additional OGlcNAcs. A tri-O-GIcNAcylated form contained all the same potential sites as detected in the di- 
O-GIcNAc form, yet it was clearer that T17 and S18 were modified with the first 2 O-GlcNAcs

with a mix of the third on S19-S21. The tetra-O-GlcNAcylated peptide was of too little abundance to site-map. Mono-phosphorylation was found largely on S13, but a mix of sites amongst T17-S20 were also detected bearing phosphorylation. Di-phosphorylated forms were again modified on S13 and on a mix of sites amongst T17-S25. Phosphorylation of S20 could be elucidated, because the fragmentation pattern indicated a strong signal at that site in addition to S13. It is probable that these two sites coexist with other sites modified at the expense of S20. The tri-phosphorylated peptide signal was too weak for sequencing. Two forms of monoO-hexosylation were partially separated chromatographically. The first contained O-hexose on S18, also a heavily modified O-GIcNAc site, and potential phosphorylation site. Separate coeluting forms were detected in mixed spectra with O-hexose amongst T17-S19. Both S18 and S19 were modified in the di-O-hexosylated form and a tri-O-hexosylated form was detected by accurate mass and elution profile only. With the exception of S13 phosphorylation, the modifications mostly occurred on the N-terminal half of the Ser/Thr-rich domain. This implies they all have potential to compete or act synergistically to influence RGA confirmation, interactions, and overall function. It was useful to also elucidate and evaluate site-locations when these three types of PTMs appeared in tandem. Peptides with mono-O-GlcNAcylation and mono-phosphorylation also contained phosphorylation S13, with O-GICNAc on T17, S18, and S19. Interestingly, with two O-GlcNAcs present, they were detected on the same residues, but phosphorylation was also detected on T17 in addition to S13. In this case, T17 appears to be a site of competition between phosphorylation and O-GIcNAcylation, potentially acting as a biochemical "switch". With mono-O-GlcNAcylation and di-phosphorylation, the O-GlcNAc was detected as a mix between T17-S20 with additional sites of phosphorylation. Again, S13, a mix of T17-S20 similar to O-GIcNAc, and also S23. While there was evidence of phosphorylation 
throughout the Ser/Thr-rich domain absent of O-GICNAc, it is possible that the sugar influences phosphorylation at certain sites, such as $\mathbf{S} 23$, by helping to more readily direct a kinase there. The addition of a third phosphorylation appears to generate a similar collection of modified sites. When mono-O-GlcNAcylation and O-hexosylation were detected in tandem, each PTM could be found on S18, S19, and S20, so all 3 residues may be sites of competition between the two PTMs. As six theoretical forms containing these two PTMs existed, it was difficult to determine which were present due to the mixed spectra, but it was certain that multiple pairs were present and these may have differing roles for RGA simply based on their location. MonoO-hexose was also detected with mono-phosphate in the absence of O-GlcNAc. Phosphorylation was detected on residues S13 and T17, consistent with that observed when mono- or di-, and di-O-GlcNAcylation was present, respectively. Interestingly, sites T17, S18, and S19 contained O-hexosylation, the same sites that contained O-GIcNAc when phosphorylation was present. Finally, mono-modified forms containing all 3 PTMs were detected in a mixture with the sites all localized to T17-S19, similar to many other modified forms of this peptides. Notably, phosphorylation on S13, consistently detected in all other phosphorylation forms, was not detected in these versions. It is possible that it was also present, but the low abundance $(<1 \%)$ of this combination of PTMs affected the ability to detect it, or the presence of both O-GIcNAc and O-hexose simultaneously in this region may have directed phosphorylation only to the Ser/Thr-rich region. In summary, this region was the most dynamically modified by all three PTMs, representing at least 30 post-translationally modified forms of RGA. This bears strong evidence that the dynamic modification of this Ser/Thr-rich region is critical for events that facilitate RGA's function(s) in plants. 
Peptide DELLAVLGYKVRSSEM contained mono-O-GIcNAcylation, and to a lesser degree, mono-O-hexosylation on S56. Site S57, the more minor site of modification relative to S56, also contained mono-O-GIcNAcylation and O-hexosylation with the latter again in lesser abundance than the former. Although these PTMs were not detected simultaneously on the Asp-N peptide, a tryptic miscleaved peptide (VRSSEMAEVALK) also containing residues S56 and S57 bore OGlcNAcylation on both residues simultaneously. There was no obvious sign of constitutive action whereby the first modification dictated the addition of the second. It was notable that on both Ser residues, O-GIcNAcylation was the major PTM and O-hexose was the minor.

The Asp-N-generated peptide, DGLSHLAT, contained mono-PTM versions of O-GIcNAc and O-hexose on S82. Again, both PTMs modified the same site and O-GICNAc was more abundant.

The majority of residue S116 was observed to be O-GlcNAcylated in peptide LSELNPPPLPASSNGL. A small amount $(<1 \%)$ of this peptide was also di-O-GlcNAcylated but was unable to be site-mapped because of inadequate levels. Residue S115 was also abundantly mono-O-hexosylated, resulting in the vast majority of this residue being occupied by 1 of these 2 modifications. Because these modifications strongly compete for the same site, residue S116 presents another likely location for a switch-like PTM event where O-GIcNAc facilitates a specific function and O-hexose facilitates a different one. Adding another level of complexity, O-hexose was also detected on S115 in the presence of S116 O-GlcNAcylation, but was not present in its absence. Similarly, a lesser amount of O-GIcNAc was detected on S115 in the presence but not the absence of S116 O-hexosylation. Therefore, modification with O-hexose or O-GlcNAc on S116 appears to direct the post-translational modification of S115. While the lone modification of S116 is in vast abundance, the lone modification of S115 by any PTM was not detected. 
Peptide SCSSPDSMTVTSTSTGTQIGK encompassed the Ser/Thr-rich domain N-terminally adjacent to the G185_G186insK lysine insertion. Following the primary sequence from the Nterminus to the C-terminus, it was the second of three such domains that were able to be modified by all three types of PTMs detected in this study. Mono-, di-, and tri-O-GlcNAcylated forms of this peptide were all detected in similar abundance. In addition, these same quantities of O-GlcNAcylation were each detected coinciding with mono-phosphorylation, and mono-OGlcNAcylation was also detected in the presence of O-hexose. The peptide also bore mono-Ohexose alone. Unfortunately, fragmentation was poor for the majority of these forms, and was not improved by the techniques already discussed. Still, several PTM forms were able to be sequenced. Mono-O-GlcNAc was detected on T176 and also T178. Less abundantly, a mixture of O-GIcNAc sites located amongst T176-S179 were detected. Mono-phosphorylation occurred on S170, several residues upstream from O-GIcNAc. One form of O-GIcNAc and phosphate was determined to bear phosphate again on S170 and O-GICNAc on T177. It was difficult to determine whether T177 was O-GlcNAcylated apart from S170 phosphorylation, as evidence for that mark was detected in mixed spectra. Given the site-location data known from this peptide, the post-translational modification of this region may be a case in which phosphorylation and OGlcNAcylation act synergistically rather than antagonistically, because they do not compete for the same Ser/Thr residue. There are quite possibly several RGA functions influenced by the post-translational modification of this domain, because like the first Ser/Thr-rich domain of RGA, it was diversely modified at $>50 \%$ level.

Immediately C-terminal to residues $167-185$ is the fourth and final poly-Ser/Thr RGA domain, encompassed by peptide GVIGTTVTTTTTTTTAAGESTR (residues 186-207). It was the most abundantly (>90\%) and second most diversely (26 PTM forms) modified peptide detected 
from RGA(+SEC). Both O-GlcNAcylation and O-hexosylation were detected. The peptide was too charge deficient to fragment well by ETD, so spectra were of insufficient quality to sitelocalize the PTMs. However, because such a large number of multiply modified forms were detected, it was possible to determine that at least six O-GIcNAc and five O-hexose sites existed on this peptide. O-GlcNAcylation was present in greater abundance than O-hexosylation overall (Figure 2.37).

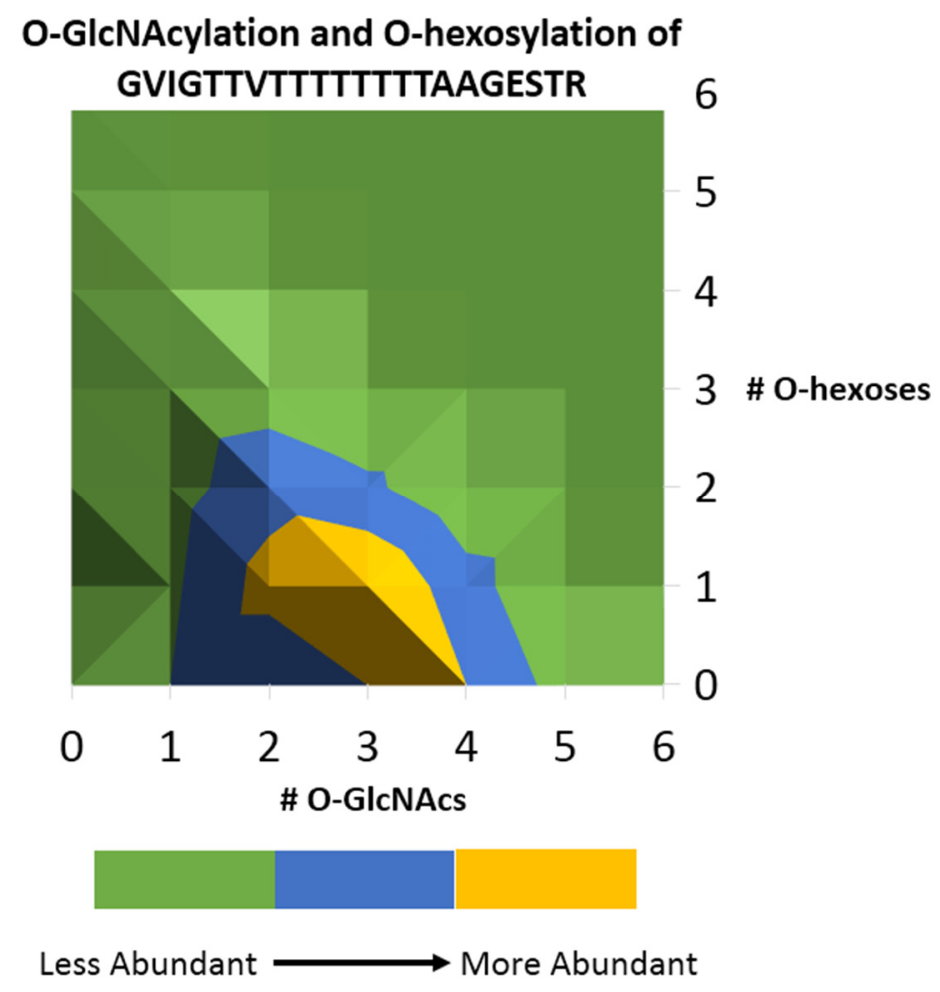

Figure 2.37 - Heat map reporting abundances of the 26 posttranslationally modified forms of GVIGTTVTTTTTTTTAAGESTR.

Peptides containing only O-GlcNAcylation were more abundant than those containing only Ohexosylation as approximately one third of the region was modified in the former and $<10 \%$ in the latter. Approximately two thirds of RGA contained combinations of both types of PTMs on this domain. Still, the abundance was skewed towards those forms containing more O-GlcNAcs relative to O-hexoses. The most abundant forms of the peptide contained 3-4 -GlcNAcs with 01 O-hexoses. Based on the observation of other abundantly and diversely modified regions of 
RGA(+SEC), it is probable that many of the PTM combinations detected on residues 186-207 exist with multiple isobaric site-location patterns. Therefore, it is reasonable to hypothesize that many more than 26 actual PTM forms of this peptide, and thus RGA, exist.

In summary, RGA was abundantly and diversely modified in this system by three separate PTMs, and one of these was a previously unknown type of nuclear protein PTM. It is already known that over 30 forms of RGA exist in this system due to its post-translational modification. Also, the 89 PTM forms summed from all the modified peptides are present in various combinations with each other to form numerous macromolecular PTM combinations. The theoretical number of PTM combinations possible are very great $(>10,000)$, so a conservative estimate for the actual number of unique RGA molecular resulting from its posttranslational modification by O-GIcNAc, phosphate, and/or O-hexose, is easily in the hundreds. This data suggests that RGA function may be intricately regulated by key PTM events, and that the enzymes responsible for catalyzing their addition and removal work together in a complex interplay. The question remains as to what is responsible for the presence of the O-hexose modification. One hypothesis was that SEC could have unknown dual activity whereby it catalyzed the addition of O-hexose from UDP-hexose substrate, analogous to O-GlcNAc from UDP-GIcNAc. The fact that all hexose sites mapped were also sites of O-GIcNAcylation support this possibility as the enzyme appears to have very similar site specificity to SEC. An alternate expression of RGA was conducted and purified to explore this possibility.

\subsubsection{The effect of SEC on O-hexosylation}

To investigate the potential role SEC plays in RGA O-hexosylation, RGA-G185_G186insK was expressed in tobacco without SEC overexpression, and the samples were analyzed by mass 
spectrometry. All three types of PTMs observed in RGA(+SEC) were also present in RGA(-SEC),

but each to a lesser overall degree in the latter (Tables $2.9 \& 2.10$ ).

\begin{tabular}{|c|c|c|c|c|c|c|c|c|c|}
\hline \multirow{3}{*}{ Peptide sequence } & \multicolumn{3}{|c|}{ \# Mods } & \multirow{3}{*}{ Sites } & \multirow{3}{*}{$\mathbf{z}$} & \multirow{3}{*}{ PPM } & \multirow{3}{*}{$\begin{array}{c}\text { Relative } \\
\text { Abundance }\end{array}$} & \multirow{2}{*}{\multicolumn{2}{|c|}{ Digest }} \\
\hline & \multirow{2}{*}{$\frac{Q}{n}$} & \multirow{2}{*}{ 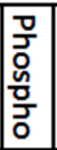 } & \multirow{2}{*}{$\begin{array}{l} \\
\mathbb{D} \\
\stackrel{x}{x} \\
0 \\
\mathscr{D} \\
\mathbb{D}\end{array}$} & & & & & & \\
\hline & & & & & & & & Trypsin & AspN \\
\hline DHHQFQGRLSNHGTgSSSSSSISK & 1 & & & S18 & 5 & 1.0 & ++ & & $\mathrm{x}$ \\
\hline DHHQFQGRLgg(SNHGTSSSSSSIS)K & 2 & & & $b c$ & 5 & 1.1 & + & & $x$ \\
\hline DHHQFQGRLp[SNHGTSSSSSSIS]K & & 1 & & d & 4 & 0.7 & ++ & & $x$ \\
\hline DHHQFQGRLSNHGp[TSpSpSSSpS]ISK & & 2 & & $\begin{array}{l}\mathrm{S} 19, \mathrm{~S} 20, \\
\mathrm{~S} 23, \&[]^{\mathrm{d}}\end{array}$ & 4 & -0.1 & + & & $x$ \\
\hline DHHQFQGRLSNHGThSSSSSSISK & & & 1 & S18 & 5 & 0.2 & + & & $x$ \\
\hline DELLAVLGYKVRg(SS)EMA & 1 & & & $\mathrm{bc}$ & 3 & 0.3 & ++ & & $x$ \\
\hline DNMLg(SELNPPPLPASS)NGL & 1 & & & c & 3 & 1.0 & +++ & & $x$ \\
\hline DNMLh(SELNPPPLPASS)NGL & & & 1 & $b c$ & 3 & 3.3 & ++ & & $x$ \\
\hline GVIGTTVg[gTIT]]TITAAGESTR & 1 & & & $\mathrm{~T} 193 \&[]^{\mathrm{d}}$ & 3 & -0.1 & ++++ & $x$ & \\
\hline GVIGTTVgg(TIIIIT)AAGESTR & 2 & & & c & 3 & -2.1 & ++ & $X$ & \\
\hline
\end{tabular}

Table 2.9 - Summary of the number of modified residues and site-locations of O-GICNAc, phosphate, and/or O-hexose PTMs on RGA(-SEC) peptides. Data is reported as in Table 2.4. 


\begin{tabular}{|c|c|c|c|c|}
\hline \multirow[b]{2}{*}{ Peptide } & \multicolumn{4}{|c|}{ Number of Forms Containing: } \\
\hline & 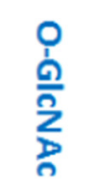 & 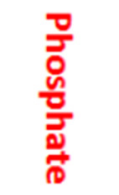 & 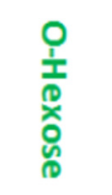 & $\stackrel{-1}{\stackrel{9}{\underline{\theta}}}$ \\
\hline DHHQFQGRLSNHGTSSSSSSISK & 2 & 3 & 1 & 6 \\
\hline DELLAVLGYKVRSSEMA & 1 & & & 1 \\
\hline DNMLSELNPPPLPASSNGL & 1 & & 1 & 2 \\
\hline \multirow[t]{4}{*}{ GVIGTTVTIIIIITTAAGESTR } & 2 & & & 2 \\
\hline & Total & Total & Total & Total \\
\hline & 6 & 3 & 2 & 11 \\
\hline & $55 \%$ & $27 \%$ & $18 \%$ & \\
\hline (h) & \multicolumn{4}{|c|}{ Abundance of Forms Containing: } \\
\hline Peptide & 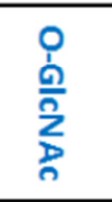 & 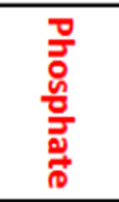 & 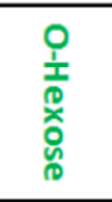 & $\stackrel{-1}{\stackrel{0}{0}}$ \\
\hline DHHQFQGRLSNHGTSSSSSSISK & ++ & ++ & + & ++ \\
\hline DELLAVLGYKVRSSEMA & ++ & & & ++ \\
\hline DNMLSELNPPPLPASSNGL & ++ & & ++ & +++ \\
\hline GVIGTTVTIIIIITIAAGESTR & ++++ & & & +++ \\
\hline
\end{tabular}

Table 2.10 - Summary of modified RGA(-SEC) peptides reporting the number and abundance of peptides containing $O$ GICNAC, phosphate, and O-hexose exclusively or in combination.

O-GIcNAcylation detected in this sample resulted from tobacco OGT activity. Notably, there was a decrease in O-hexosylation that was comparable to the decrease in O-GlcNAcylation, implying that SEC does in fact catalyze O-hexose addition. However, this conclusion must be taken with caution as well, because if these PTMs do form a complex interplay with each other and their respective catalyzing enzymes, SEC down-regulation may still have been indirectly suppressing the function of an independent O-hexose transferase. For example, phosphorylation was also 
decreased in overall abundance primarily due to the lack of any phosphorylation signal detected on peptide SCSSPDSMVTSTSTGTQIGK, so a change in SEC expression appeared to alter kinase activity and specificity.

A total of four peptides, all modified in RGA(+SEC), were detected with PTMs in the RGA(-SEC) sample. Three of these four were the most abundantly modified peptides in $\mathrm{RGA}(+\mathrm{SEC})$ and all four were in the top five most abundantly modified as well. The number of RGA(-SEC) PTMs discovered totaled 11 vs. 43 found on RGA(+SEC), and these consisted of 60 GlcNAcs, 3 phosphates, and 2 O-hexoses. Of these, 6 were able to be localized (Table 2.11).

\begin{tabular}{|c|c|c|c|}
\hline 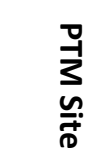 & 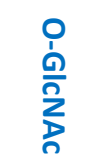 & 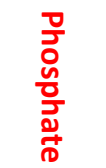 & 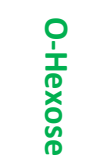 \\
\hline S18 & $x$ & & $x$ \\
\hline S19 & & $x$ & \\
\hline S20 & & $x$ & \\
\hline S23 & & $x$ & \\
\hline \multirow[t]{3}{*}{ T193 } & $x$ & & \\
\hline & Total & Total & Total \\
\hline & 2 & 3 & 1 \\
\hline
\end{tabular}

Table 2.11 - PTM Sites unambiguously localized on RGA(-SEC).

All PTM sites were located in regions previously detected to be modified although two PTM sites not localized in RGA(+SEC), S19 phosphorylation and T193 O-GlcNAcylation, were able to be unambiguously site-mapped in RGA(-SEC). Multiple PTMs were detected on three of the four peptides, but not to the same scope as RGA(+SEC) and PTMs of different types were not simultaneously present on the same molecules as they were with the SEC overexpression.

Residues 4-26 only contained 6 detected PTM forms vs. 30 previously. The overall abundance of O-GIcNAc and O-hexose were also reduced by an order of magnitude. RGA(-SEC) 
contained S18 mono-O-GIcNAcylation, 1 of 2 most abundant O-GIcNAc sites previously detected. A di-O-GlcNAcylated form at $<1 \%$ was also detected. Mono-O-hexosylation was also again localized to S18 as before. Mono-phosphorylation was detected in similar abundance to S18 OGIcNAcylation, although MS/MS spectra revealed a mix of many phosphorylation sites that ranged the entire poly-Ser/Thr domain. It is possible that the decrease in O-GIcNAcylation allowed kinases to adopt less stringent specificity in modifying this domain. Spectra resulting from di-phosphorylation signal also revealed a mix of sites. Three of these phosphorylation sites were able to be localized: S19, S20, and S23.

Mono-O-GIcNAcylation was detected on peptide DELLAVLGYKVRSSEMA, formed from conventional AspN cleavage $\mathrm{N}$-terminally to D44 and an $\mathrm{N}$-terminal E61 cleavage. Asp- $\mathrm{N}$ is known to bear some activity towards Glu residues albeit it is generally lower than for Asp ${ }^{84}$. The two modifiable residues contained in this peptide, S56 and S57, were both modified with OGIcNAc and O-hexose in RGA(+SEC). RGA(-SEC) S56/57 O-GlcNAcylation abundance was decreased relative to $\mathrm{RGA}(+\mathrm{SEC})$ and O-hexosylation was undetected.

AspN peptide DNMLSELNPPPLPASSNGL containing S105, S115, and S116 was mono-OGIcNAcylated and O-hexosylated to lesser degrees in RGA(-SEC) than RGA(+SEC). Also, these PTMs were not simultaneously detected in the former as they were in the latter.

Lastly, the Thr-rich peptide, GVIGTTVTTTTTTTTAAGESTR, was found to contain monoand di-O-GlcNAcylation. While the O-GIcNAc abundance as a whole was reduced on this domain, the level of mono- and di-O-GIcNAcylation was increased on RGA(-SEC) relative to RGA(+SEC). O-GIcNAcylation was not detected in a series of up to 6 simultaneous modifications like in RGA(+SEC). Also, O-hexose was not detected on this peptide in RGA(-SEC) at all, contrasting with $\mathrm{RGA}(+\mathrm{SEC})$ in which $>50 \%$ of the region contained a combination of $\mathrm{O}-\mathrm{GlcNAC}$ 
and O-hexose PTMs present together. Instead, in RGA(-SEC), OGT catalyzed the addition of 1 or 2 O-GICNAcs on 1 quarter of RGA's poly-Thr domain, and successive additions either did not occur or were rapidly removed by O-GIcNAcase. ETD spectral quality of mono-O-GIcNAcylated GVIGTTVTTTTTTTTAAGESTR moderately improved for RGA(-SEC), presumably because this PTM form was present in higher abundance than any single PTM form detected in RGA(+SEC). As a result, O-GlcNAc was able to be localized to one site: T193, and evidence demonstrated these spectra were mixed, so other sites existed on the poly-Thr domain as well (Figure 2.38).

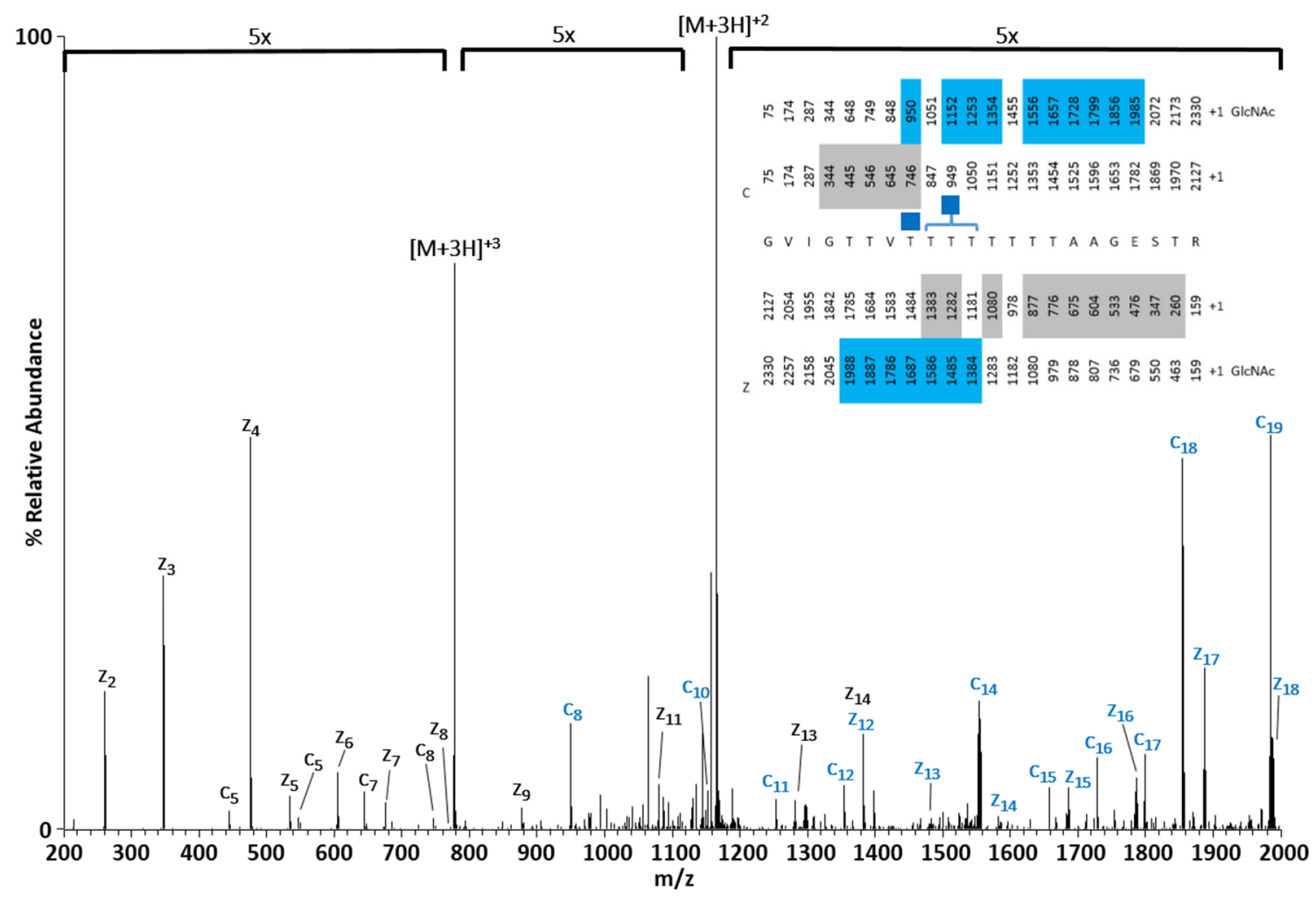

Figure 2.38 - Mixed ETD spectrum of mono-O-GIcNAcylated GVIGTTVTTTTTTTTTAAGESTR in RGA(-SEC). A GICNAC was localized to T193 and one or more were detected between T194-T196.

\section{Summary and Future Analysis related to O-hexose}

This analysis of RGA(-SEC) revealed that O-GIcNAcylation, O-hexosylation, and phosphorylation are all part of the repertoire of RGA PTMs, whether in the presence or the absence of overexpressed OGT, SEC. By comparing the locations and scale of RGA(-SEC) post- 
translational modification to RGA(+SEC), we find that OGT expression level has a direct effect on the levels and scope of O-GIcNAcylation, phosphorylation, and O-hexosylation. Given that the relative decrease in O-hexose levels tracked closely with those of O-GICNAc, it is clear that SEC has either an indirect if not direct effect on O-hexosylation. Indirectly, O-GlcNAcylation may be a positive regulator of one or more O-hexose transferases, or a negative regulator of one or more glycases responsible for O-hexose removal. Alternatively, this data bears strong evidence in support of SEC having a more direct role in O-hexosylation. The level of O-hexose decreased similarly to that of O-GICNAc and O-hexose was only found in regions also found to be OGIcNAcylated. In addition, every O-hexose site localized (whether subjected to SEC overexpression or not) was also determined to be an O-GlcNAc site. This strongly implies that SEC catalyzes the addition of O-hexose, or that it first modifies RGA with O-GIcNAc, and N-acetyl groups are subsequently removed from a portion of the O-GIcNAc moieties. All three of the cases above add an additional layer of complexity to the current understanding of what PTMs occur on nuclear and cytoplasmic proteins, and how those PTMs alter the confirmation, function, localization, etc. of those proteins.

Future studies will need to focus on more fully characterizing RGA O-hexose in 3 ways: (1) determining the exact type(s) of hexose sugar, (2) determining what enzyme(s) and processes are responsible for its addition, and (3) probing its effects on RGA function in the context of RGA O-GIcNAcylation and phosphorylation. The scope of the third question is especially vast. To answer the first question, enzymatic glyco-labeling experiments may be performed in which a known control hexose, such as glucose, is labeled with a radioisotope or fluorescent sugar by an appropriate glycosyltransferase, allowing detection of a successful transfer to the acceptor hexose. If RGA hexose responded accordingly, controlling for false 
positive response from O-GIcNAc, one may claim identification of the hexose sugar type. Mass spectrometric analysis would provide a direct and an even more accurate assessment of the results and would allow one to determine the exact O-hexose moieties modified (allowing distinction between two or more types of O-hexoses if they existed). For example, galactosyltransferase, Gal-T6, transfers galactose to glucose on glucosylceramide to synthesize lactosylceramide ${ }^{85}$. Gal-T6 could be used to probe for glucose on RGA in this manner. If the hexose moiety was glucose, it would be an important insight, but would still leave the question of OGT's role unanswered. In an attempt to probe that question, both plant OGTs may be silenced to determine if $O$-hexose is still present on RGA. This method may be difficult to interpret if O-hexose were subsequently undetected though, because this RGA study has already shown that OGT expression has a drastic effect on the level of O-hexosylation, so its disappearance after OGT silencing could still be an indirect affect due to the lack of O-GIcNAc. Also, elimination of both OGTs has previously been demonstrated to have serious negative effects on plant development and vitality ${ }^{39}$, so this could be unviable in practice. Much further exploration of this previously unreported nuclear O-hexose PTM will be required to gain a holistic characterization of its function on RGA, and its potential abundance and function in other proteins from plants or other kingdoms.

\subsubsection{Phosphorylation of RGA by CK1}

Multiple phosphorylation sites detected in RGA(+SEC) fit the casein kinase 1 (CK1) motifs, Ser-X-X-Ser/Thr or Ser/Thr-X-X-X-Ser. To further investigate the existence of phosphorylation and probe its effect on overall RGA post-translational modification, a third RGA sample was overexpressed with CK1 in tobacco. Samples digested with Asp-N or trypsin were first screened, then enriched for phosphorylation by IMAC as described previously. In all, 24 
O-hexoses (Tables 2.12\&2.13).

\begin{tabular}{|c|c|c|c|c|c|c|c|c|c|c|c|}
\hline \multirow{3}{*}{ Residues } & \multirow{3}{*}{ Peptide sequence } & \multicolumn{3}{|c|}{ \# Mods } & \multirow{3}{*}{ Sites } & \multirow{3}{*}{$z$} & \multirow{3}{*}{ PPM } & \multirow{3}{*}{$\begin{array}{c}\text { Relative } \\
\text { Abundance }\end{array}$} & \multirow{2}{*}{\multicolumn{2}{|c|}{ Digest }} & \multirow{3}{*}{ § } \\
\hline & & \multirow{2}{*}{$\frac{0}{n}$} & \multirow{2}{*}{ 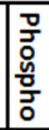 } & \multirow{2}{*}{ 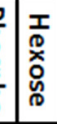 } & & & & & & & \\
\hline & & & & & & & & & Trypsin & AspN & \\
\hline $4-26$ & DHHQFQGRLSNHGg[TgSSgSS]SSISK & 1 & & & $\begin{array}{c}\mathrm{S} 18, \mathrm{~S} 20, \& \\
{[]^{\mathrm{d}}}\end{array}$ & 5 & -1.0 & ++ & & $\mathrm{x}$ & \\
\hline $4-26$ & DHHQFQGRLgg(SNHGTSSSSSSIS)K & 2 & & & $\mathrm{bc}$ & 5 & -1.9 & + & & $x$ & \\
\hline $4-26$ & DHHQFQGRLSNHGTSSSSSpSISK & & 1 & & $\mathrm{~S} 23$ & 5 & -1.0 & ++ & & $x$ & \\
\hline $4-26$ & DHHQFQGRLp[SNHGTSSSSSSIS]K & & 1 & & d & 5 & -1.9 & + & & $x$ & $\mathrm{x}$ \\
\hline $4-26$ & DHHQFQGRLSNHGTSpSpSSSSISK & & 1 & & S19 \& S20 & 5 & 2.9 & ++ & & $\mathrm{x}$ & $\mathrm{x}$ \\
\hline $4-26$ & DHHQFQGRLSNHGTpSpSSSp[SSIS]K & & 2 & & $S 18, S 19 \&[]^{d}$ & 5 & 0.3 & + & & $\mathrm{x}$ & $\mathrm{x}$ \\
\hline $4-26$ & DHHQFQGRLSNHGTSpSpSSp[SSIS]K & & 2 & & $\mathrm{~S} 19, \mathrm{~S} 20 \&[]^{\mathrm{d}}$ & 5 & 1.4 & ++ & & $x$ & $x$ \\
\hline $4-26$ & DHHQFQGRLppp[SNHGTSSSSSSIS]K & & 3 & & d & 5 & -0.5 & + & & $x$ & $\mathrm{x}$ \\
\hline $4-26$ & DHHQFQGRLpppp(SNHGTSSSSSSIS)K & & 4 & & c & 4 & 0.5 & + & & $x$ & $x$ \\
\hline $4-26$ & DHHQFQGRLSNHGThSSSSSSISK & & & 1 & $\mathrm{~S} 18$ & 5 & -1.5 & + & & $x$ & \\
\hline $44-60$ & DELLAVLGYKVRg(SS)EMA & 1 & & & $b c$ & 3 & -1.3 & + & & $\mathrm{x}$ & \\
\hline $44-60$ & DELLAVLGYKVRpSSEMA & & 1 & & S56 & 3 & 3.5 & + & & $\mathrm{x}$ & $\mathrm{x}$ \\
\hline $101-119$ & DNMLSELNPPPLPAg(SS)NGL & 1 & & & c & 2 & 0.5 & n+r+ & & $x$ & \\
\hline $101-119$ & DNMLp(SELNPPPLPASS)NGL & & 1 & & $b c$ & 2 & -6.0 & ++ & & $x$ & \\
\hline $101-119$ & DNMLh(SELNPPPLPASS)NGL & & & 1 & bc & 2 & -0.5 & ++ & & $x$ & \\
\hline $140-164$ & VIPGNAIYQFPAIDp(SSS)SSNNQNKR & & 1 & & c & 4 & 1.6 & ++ & $x$ & & $\mathrm{x}$ \\
\hline $167-185$ & $\mathbf{g}($ SCSSPDSMVTSTSTGT)QIGK & 1 & & & $\mathrm{bc}$ & 2 & 2.3 & + & $x$ & & \\
\hline $167-185$ & p(SCSSPDSMVT)STSTGTQIGK & & 1 & & c & 3 & 0.2 & ++ & $x$ & & $\mathrm{x}$ \\
\hline $186-207$ & GVIGTTVgTIIIIITIAAGESTR & 1 & & & T193 & 3 & 5.4 & +++ & $x$ & & \\
\hline $186-207$ & GVIGTTVg[TIIT]TITAAGESTR & 1 & & & d & $4^{\mathrm{g}}$ & 1.2 & $++++^{\mathrm{g}}$ & $x$ & & \\
\hline $186-207$ & GVIGh(TTVTTTTTTTTAAGEST)R & & & 1 & $b c$ & 3 & 2.7 & + & $x$ & & \\
\hline
\end{tabular}

Table 2.12 - Summary of the number of modified residues and site-locations of O-GlcNAc, phosphate, and/or O-hexose PTMs on RGA(+CK1). Data is reported as in Table 2.4. 


\begin{tabular}{|c|c|c|c|c|}
\hline \multicolumn{5}{|c|}{ PTM Forms of RGA(CK1) } \\
\hline \multirow[b]{2}{*}{ Peptide } & \multicolumn{4}{|c|}{ Number of Forms Containing: } \\
\hline & 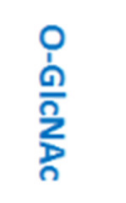 & 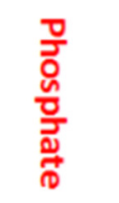 & 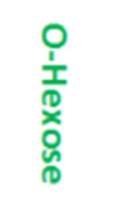 & $\begin{array}{l}\overrightarrow{0} \\
\stackrel{\mathbb{D}}{=}\end{array}$ \\
\hline DHHQFQGRLSNHGTSSSSSSISK & 3 & 8 & 1 & 12 \\
\hline DELLAVLGYKVRSSEMA & 1 & 1 & & 2 \\
\hline DNMLSELNPPPLPASSNGL & 1 & 1 & 1 & 3 \\
\hline VIPGNAIYQFPAIDSSSSSNNQNKR & & 1 & & 1 \\
\hline SCSSPDSMVTSTSTGTQIGK & 1 & 1 & & 2 \\
\hline \multirow[t]{4}{*}{ GVIGTTVTTTTTTTTAAGESTR } & 2 & & 1 & 3 \\
\hline & Total & Total & Total & Total \\
\hline & 8 & 12 & 4 & 24 \\
\hline & $33 \%$ & $50 \%$ & $17 \%$ & \\
\hline \multicolumn{5}{|c|}{ PTM Forms of RGA(CK1) } \\
\hline & \multicolumn{4}{|c|}{ Abundance of Forms Containing: } \\
\hline Peptide & 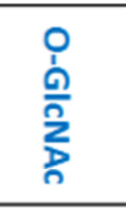 & $\begin{array}{l}\text { 뭄 } \\
\text { 음 } \\
\frac{n}{0} \\
\frac{7}{0} \\
\frac{0}{0} \\
\end{array}$ & 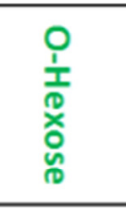 & $\begin{array}{l}\stackrel{-1}{\circ} \\
\stackrel{\Xi}{\underline{\Xi}}\end{array}$ \\
\hline DHHQFQGRLSNHGTSSSSSSISK & ++ & +++ & + & +++ \\
\hline DELLAVLGYKVRSSEMA & + & + & & + \\
\hline DNMLSELNPPPLPASSNGL & ++++ & ++ & ++ & ++++ \\
\hline VIPGNAIYQFPAIDSSSSSNNQNKR & & ++ & & ++ \\
\hline SCSSPDSMVTSTSTGTQIGK & + & ++ & & ++ \\
\hline GVIGTTVTTTTTTTTAAGESTR & +++ & & & +++ \\
\hline
\end{tabular}

Table 2.13 - Summary of modified $R G A(+C K 1)$ peptides reporting the number and abundance of peptides containing $O$ GIcNAc, phosphate, and O-hexose. No combinations of different PTM types were detected.

As in RGA(-SEC), multiply modified peptides were detected, but they only contained PTMs of the same type. The number and overall abundance of O-GIcNAcs and O-hexoses decreased relative to $R G A(+S E C)$. However, $R G A(+C K 1)$ phosphorylation increased relative to $R G A(+S E C)$ on 4 
peptides, and the number of phosphorylation sites detected more than doubled to 12 . Of these, 9 PTMs were unambiguously localized (Table 2.14).

\begin{tabular}{|c|c|c|c|}
\hline 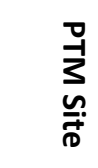 & 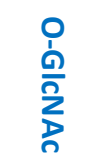 & 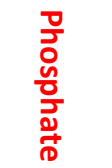 & 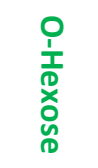 \\
\hline S18 & $x$ & $x$ & $x$ \\
\hline S19 & & $x$ & \\
\hline S20 & $x$ & $x$ & \\
\hline S23 & & $x$ & \\
\hline S56 & & $x$ & \\
\hline T193 & $x$ & & \\
\hline & Total & Total & Total \\
\hline & 3 & 5 & 1 \\
\hline
\end{tabular}

Table 2.14 - PTM Sites unambiguously localized on RGA(+CK1).

Two phosphorylation sites undetected in RGA(+SEC) and RGA(-SEC) were found in RGA(CK1). A small amount was detected on S18, a minor site of O-hexosylation and major site of O-

GIcNAcylation in RGA(+SEC). In addition, S56, also modifiable with O-GIcNAc and O-hexose, was found to contain phosphorylation.

Peptide DHHQFQGRLSNHGTSSSSSSISK was detected with 12 PTM forms, 8 of which were phosphorylated. Evidence pointed to a wide mix of phosphorylation sites with much of the ETD spectra mixed. Also, a tetra-phosphorylated form was detected in RGA(CK1), whereas only up to tri-phosphorylation had been previously detected. Site S18 was only unambiguously detected in a di-phosphorylated form. This may indicate that S19 or a site near the C-terminus of the peptide facilitates the addition of phosphorylation to S18. This cannot be certain though, because a wide mix of mono-phosphorylation sites were also detected and could include site S18. The abundance of phosphorylation in this Poly-Ser/Thr region increased over that of 
$\mathrm{RGA}(+\mathrm{SEC})$ overall. Also, a tetra-phosphorylated form was detected, when only up triphosphorylation was previously detected in this region.

A small amount of phosphorylation was detected on DELLAVLGYKVRSSEMA at S56, a peptide previously only detected bearing O-GlcNAcylation and O-hexosylation. Phosphorylation signal was uniquely detected on RGA(CK1) peptide DNMLSELNPPPLPASSNGL as well, a region already detected as O-GIcNAcylated and O-hexosylated. This furthers the hypothesis that these three PTM types and their corresponding enzymes all have an interplay in regulating RGA.

CK1 overexpression had an interesting effect on the poly-Ser/Thr region contained in peptide SCSSPDSMVTSTSTGTQIGK. In RGA(-SEC), phosphorylation levels were undetectable, but $R G A(+C K 1)$ restored them to levels comparable with $R G A(+S E C)$. This indicates that SEC and CK1 act in a synergetic manner to increase phosphorylation of this region.

Overexpression of RGA along with CK1 allowed the detection of two additional phosphorylated peptides, and two additional phosphorylation sites compared to RGA(+SEC). It also clearly demonstrated that CK1 is an enzyme responsible for modifying RGA in at least four different regions of the protein.

\subsection{Conclusions}

Comprehensively, 92 distinct forms of modified peptides were detected on RGA, comprised of at least 46 PTM sites. In all, 32 of these modifications were able to be unambiguously localized (Table 2.15). 


\begin{tabular}{|c|c|c|c|}
\hline \multicolumn{4}{|c|}{$\begin{array}{c}\text { Comprehensive Site-Localized RGA } \\
\text { PTMs }\end{array}$} \\
\hline 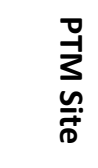 & 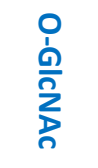 & 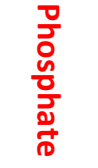 & 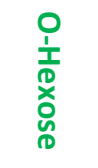 \\
\hline S13 & & $x$ & \\
\hline T17 & $x$ & $x$ & $x$ \\
\hline S18 & $x$ & $x$ & $x$ \\
\hline S19 & $x$ & $x$ & $x$ \\
\hline S20 & $x$ & $x$ & $x$ \\
\hline S23 & & $x$ & \\
\hline S56 & $x$ & $x$ & $x$ \\
\hline S57 & $x$ & & $x$ \\
\hline S82 & $x$ & & $x$ \\
\hline S115 & $x$ & & $x$ \\
\hline S116 & $x$ & & $x$ \\
\hline S170 & & $x$ & \\
\hline T176 & $x$ & & \\
\hline T177 & $x$ & & \\
\hline T178 & $x$ & & \\
\hline T193 & $x$ & & \\
\hline S253 & $X$ & & \\
\hline S537 & $x$ & & \\
\hline & Total & Total & Total \\
\hline & 15 & 8 & 9 \\
\hline
\end{tabular}

Table 2.15 - Comprehensive list of PTMs localized between RGA(+SEC), RGA $(-S E C)$, and RGA(+CK1)

Of the 15 O-GIcNAc sites identified on RGA, 9 were also sites of O-hexosylation. Of

these, 5 were also sites of phosphorylation. Interestingly, all sites that were able to be modified by O-GIcNAc and phosphate were also O-hexose sites. Over 30 post-translationally modified forms of RGA may exist in a single plant, and these data suggest an interplay amongst all three of these modifications on RGA is likely critical to its proper function as a negative regulator of plant development (Figures 2.39 \& 2.40). 
PTM Abundance: RGA(+SEC) vs RGA(-SEC) vs RGA(+CK1):

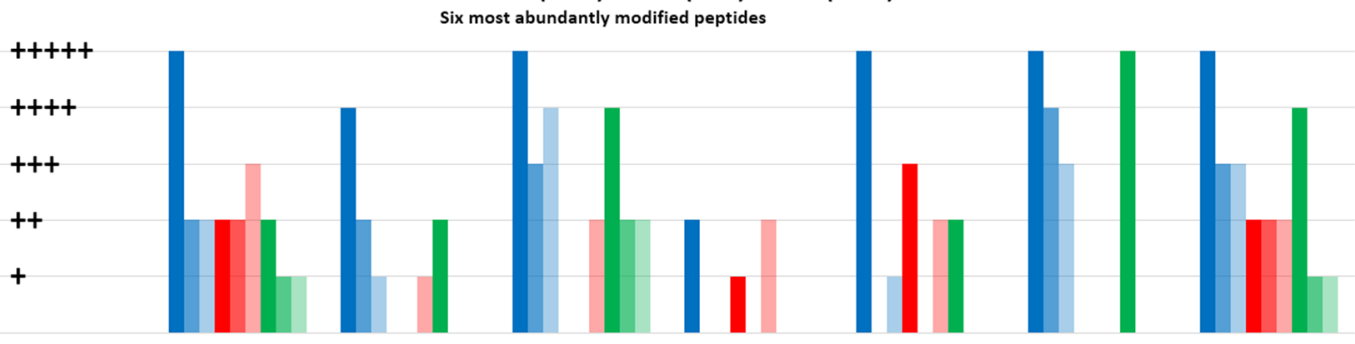

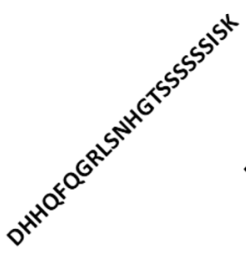

Forms containing: O-GIcNAc (+SEC) - Forms containing: Phosphate (+SEC) arms containing: 0 -Hexose $(+\mathrm{SEC})$

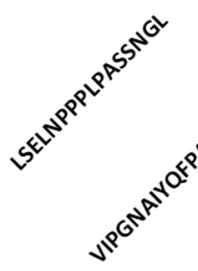

Forms containing: O-GICNAC (-SEC) - Forms containing: Phosphate (-SEC) - Forms containing: O-Hexose (-SEC)
Forms containing: O-GlcNAC (+CK1)

Forms containing: Phosphate (+CK1)

Forms containing: O-Hexose (+CK1)

Figure 2.39 - Sum total of O-GICNAC, phosphate, and O-hexose in RGA(+SEC,-SEC,\&+CK1) for the six most abundantly modified RGA peptides. 
(a) RGA (+SEC)

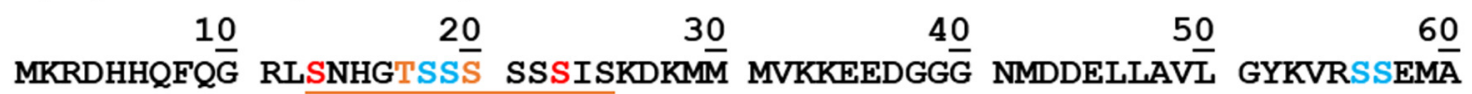

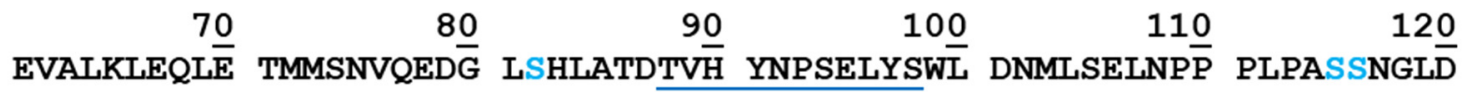

$\begin{array}{llllll}13 \underline{0} & 14 \underline{0} & 15 \underline{0} & 16 \underline{0} & 17 \underline{0} & 18 \underline{0}\end{array}$

PVLPSPEICG FPASDYDLKV IPGNAIYQFP AIDSSSSSNN QNKRLKSCS $\bar{S}$ PDSMVTSTST

$19 \underline{0} 20 \underline{0} 21 \underline{0} \quad 220-230 \quad 240$

GTQIGGVIGT TVTTTTTTTT AAGESTRSVI LVDSQENGVR LVHALMACA $\bar{E}$ AIQQNNLTL $\bar{A}$

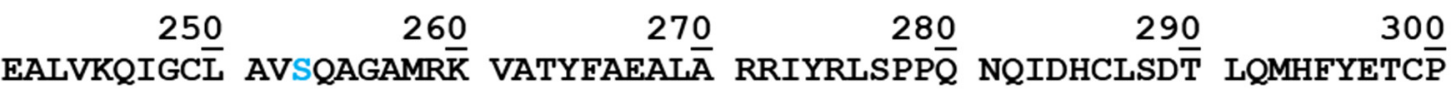

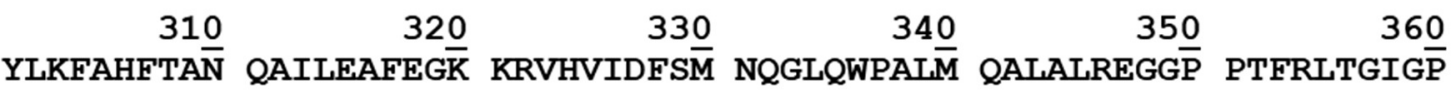

$\begin{array}{llllll}370 & 380 & 390 & 400 & 410 & 420\end{array}$

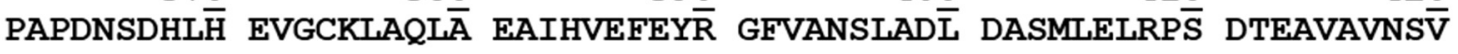

$\begin{array}{llllll}430 & 440 & 450 & 460 & 470 & 480\end{array}$

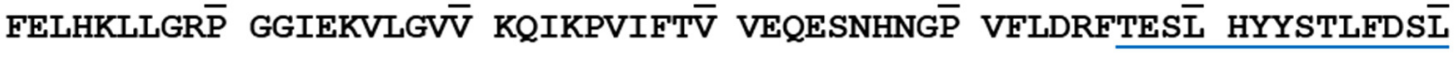

$49 \underline{0} \quad 50 \underline{0} \quad 5100520050$

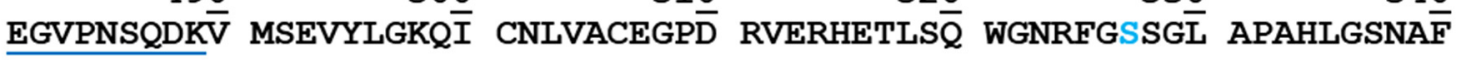

$55 \underline{0} \quad 56 \underline{0} \quad 57 \underline{0} \quad 58 \underline{0}$

KQASMLLSVF NSGQGYRVEE SNGCLMLGWH TRPLITTSA $\overline{\bar{W}}$ KLSTAAY 


\section{(b) RGA (+CK1)}

$\begin{array}{rrrrr}10 & 20 & 30 & 40 & 50\end{array}$

$\begin{array}{llllll}7 \underline{0} & 8 \underline{0} & 90 & 10 \underline{0} & 110 & 120\end{array}$

EVALKLEQL $\bar{E}$ TMMSNVQEDG $\bar{G}$ LSHLATDTV $\bar{H}$ YNPSELYSWL DNMLSELNP $\bar{P}$ PLPASSNGL $\bar{D}$

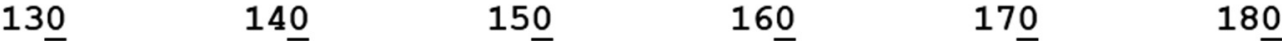

PVLPSPEICG FPASDYDLKV IPGNAIYQFP AIDSSSSSNN QNKRLKSCSS PDSMVTSTST

$190 \quad 200 \quad 210 \quad 220 \quad 230 \quad 240$

GTQIGGVIGT TVTTTTTTTT AAGESTRSVI LVDSQENGVR LVHALMACA $\bar{E}$ AIQQNNLTL $\bar{A}$

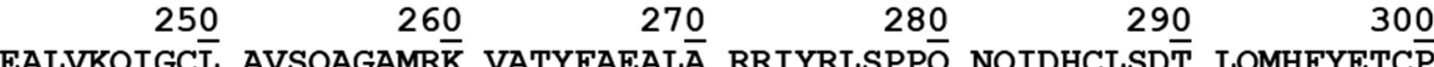

DRT

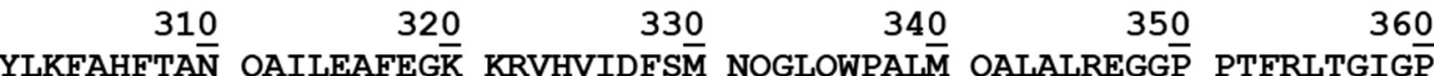

YLKFAHFTAN QAILEAFEGK KRVHVIDFSM NQGLQWPALM QALALREGGP PTFRLTGIGP

$370 \quad 380-390 \quad 400 \quad 410 \quad 420$

PAPDNSDHL $\overline{\mathrm{H}}$ EVGCKLAQL $\overline{\mathrm{A}}$ EAIHVEFEYR GFVANSLAD $\overline{\mathrm{L}}$ DASMLELRP $\overline{\mathrm{S}}$ DTEAVAVNS $\overline{\mathrm{V}}$

$\begin{array}{rrrrrr}430 & 440 & 450 & 460 & 470 & 480 \\ \text { FELHKLLGRP } & \text { GGIEKVLGVV } & 40 \text { KOIKPVIFT' } & \text { VEOESNHNGP } & \text { VFLDRFTESL } & \text { HYYSTLFDSI }\end{array}$

$490 \quad 500 \quad 510 \quad 520 \quad 530 \quad 540$

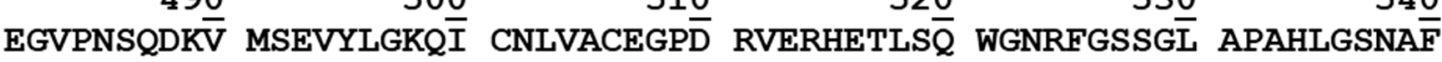

$\begin{array}{rrrr}550 & 560 & 570 & 580 \\ \text { KQASMLLSVF } & \text { NSGQGYRVEE } & \text { SNGCLMLGWH } & \text { TRPLITTSAW } \\ \text { KLSTAAY }\end{array}$ 


\section{(c) RGA (-SEC)}

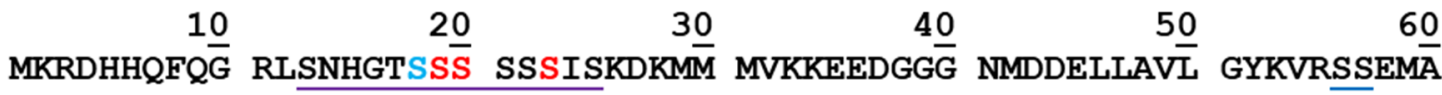
$\begin{array}{rrrrrr}\frac{70}{2} & 80 & 90 & 100 & 110 & 120 \\ \text { EVALKLEQLE } & \text { TMMSNVQEDG } & \text { LSHLATDTVH } & \text { YNPSELYSWL } & \text { DNMLSELNPP } & \text { PLPASSNGLD }\end{array}$

$13 \underline{0} \quad 14 \underline{0} \quad 15 \underline{0} \quad 16 \underline{0} \quad 17 \underline{0} \quad 18 \underline{0}$

PVLPSPEICG FPASDYDLKV IPGNAIYQF $\bar{P}$ AIDSSSSSNN QNKRLKSCS $\bar{S}$ PDSMVTSTST

$19 \underline{0} 20 \underline{0} \quad 21 \underline{0} \quad 22 \underline{0} \quad 23 \underline{0} \quad 24 \underline{0}$

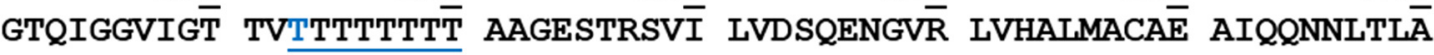

$250 \quad 260 \quad 27 \underline{0} \quad 28 \underline{0} \quad 29 \underline{0} \quad 30 \underline{0}$

EALVKQIGC $\bar{L}$ AVSQAGAMR $\bar{K}$ VATYFAEAL $\bar{A}$ RRIYRLSPP $\bar{Q}$ NQIDHCLSD $\bar{T}$ LQMHFYETC $\bar{P}$

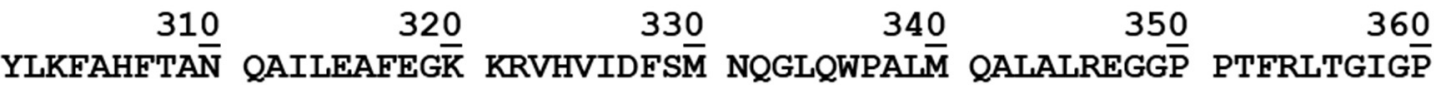

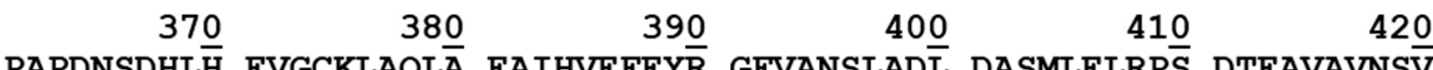

PAPDNSDHL $\bar{H}$ EVGCKLAQL $\bar{A}$ EAIHVEFEYR GFVANSLAD $\bar{L}$ DASMLELRPS DTEAVAVNSV

$\begin{array}{rrrrrr}430 & 440 & 450 & 460 & 470 & 480 \\ \text { FELHKLLGRP } & \text { GGIEKVLGVV } & \text { KQIKPVIFT'V } & \text { VEQESNHNGP } & \text { VFLDRFTEST } & \text { HYYSTLFDS } \bar{L}\end{array}$

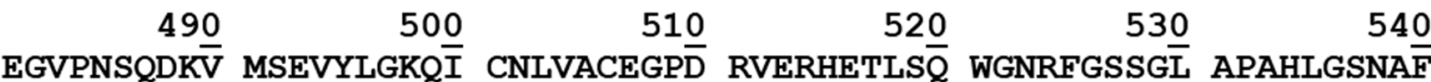

$\begin{array}{rrrr}550 & 560 & 570 & 580 \\ \text { KQASMLLSVF } & \text { NSGQGYRVEE } & \text { SNGCLMLGWH } & \text { TRPLITTSA WT KLSTAAY }\end{array}$

Figure 2.40 - RGA sequences summarizing residues modified with O-GIcNAc, phosphate, O-hexose, O-GIcNAc \& phosphate, O-GICNAC \& O-hexose, phosphate \& O-hexose, and O-GICNAC, phosphate, \& O-hexose PTMs on (a) $R G A(+S E C)$, (b) RGA(-SEC+CK1), and (c) RGA(-SEC-CK1). Underlines are color-coded as above to indicate regions in which one or more PTMs have been detected by accurate mass and elution profile.

This study comprises the most complete characterization of post-translational

modifications on any O-GIcNAcylated plant protein to date. It identifies numerous sites of OGlcNAc, phosphate, and the previously undescribed nuclear PTM, O-hexose, while demonstrating that these PTMs have a complex interplay determining how they collectively contribute to RGA function. 


\subsection{References}

1. Creasy, D. M. \& Cottrell, J. S. Unimod: Protein modifications for mass spectrometry. Proteomics 4, 1534-1536 (2004).

2. Farley, A. R. \& Link, A. J. Identification and quantification of protein posttranslational modifications. Methods Enzymol. 463, 725-763 (2009).

3. Manning, G., Whyte, D. B., Martinez, R., Hunter, T. \& Sudarsanam, S. The protein kinase complement of the human genome. Science 298, 1912-1934 (2002).

4. Hubbard, M. J. \& Cohen, P. On target with a new mechanism for the regulation of protein phosphorylation. Trends Biochem. Sci. 18, 172-177 (1993).

5. Cohen, P. The role of protein phosphorylation in human health and disease. European Journal of Biochemistry 268, 5001-5010 (2001).

6. White, F. M. Quantitative phosphoproteomic analysis of signaling network dynamics. Curr. Opin. Biotechnol. 19, 404-409 (2008).

7. Hart, G. W., Slawson, C., Ramirez-Correa, G. \& Lagerlof, O. Cross talk between OGlcNAcylation and phosphorylation: roles in signaling, transcription, and chronic disease. Annu. Rev. Biochem. 80, 825-858 (2011).

8. Torres, C. R. \& Hart, G. W. Topography and polypeptide distribution of terminal Nacetylglucosamine residues on the surfaces of intact lymphocytes. Evidence for O-linked GlcNAc. J. Biol. Chem. 259, 3308-3317 (1984).

9. Ngoh, G. A., Facundo, H. T., Zafir, A. \& Jones, S. P. O-GlcNAc Signaling in the Cardiovascular System. Circulation Research 107, 171-185 (2010).

10. Ruan, H., Singh, J. P., Li, M., Wu, J. \& Yang, X. Cracking the O-GlcNAc code in metabolism. Trends in Endocrinology \& Metabolism 24, 301-309 (2013).

11. Hart, G. W., Slawson, C., Ramirez-Correa, G. \& Lagerlof, O. Cross talk between OGlcNAcylation and phosphorylation: roles in signaling, transcription, and chronic disease. Annu. Rev. Biochem. 80, 825-858 (2011).

12. Nolte, D. \& Muller, U. Human O-GIcNAc transferase (OGT): genomic structure, analysis of splice variants, fine mapping in Xq13.1. Mamm. Genome 13, 62-64 (2002).

13. Lazarus, M. B., Nam, Y., Jiang, J., Sliz, P. \& Walker, S. Structure of human O-GlcNAc transferase and its complex with a peptide substrate. Nature 469, 564-567 (2011).

14. Das, A. K., Cohen, P. T. W. \& Barford, D. The structure of the tetratricopeptide repeats of protein phosphatase 5: implications for TPR-mediated protein-protein interactions. EMBO J. 17, 1192-1199 (1998).

15. Cheung, W. D., Sakabe, K., Housley, M. P., Dias, W. B. \& Hart, G. W. O-linked beta-Nacetylglucosaminyltransferase substrate specificity is regulated by myosin phosphatase targeting and other interacting proteins. J. Biol. Chem. 283, 33935-33941 (2008). 
16. Gao, Y., Wells, L., Comer, F. I., Parker, G. J. \& Hart, G. W. Dynamic O-glycosylation of nuclear and cytosolic proteins: cloning and characterization of a neutral, cytosolic beta-Nacetylglucosaminidase from human brain. J. Biol. Chem. 276, 9838-9845 (2001).

17. Toleman, C., Paterson, A. J., Whisenhunt, T. R. \& Kudlow, J. E. Characterization of the Histone Acetyltransferase (HAT) Domain of a Bifunctional Protein with Activable O-GlcNAcase and HAT Activities. J. Biol. Chem. 279, 53665-53673 (2004).

18. Comtesse, N., Maldener, E. \& Meese, E. Identification of a Nuclear Variant of MGEA5, a Cytoplasmic Hyaluronidase and a $\beta-\mathrm{N}-$-Acetylglucosaminidase. Biochem. Biophys. Res. Commun. 283, 634-640 (2001).

19. Kim, E. J., Kang, D. O., Love, D. C. \& Hanover, J. A. Enzymatic characterization of O-GlcNAcase isoforms using a fluorogenic GlcNAc substrate. Carbohydr. Res. 341, 971-982 (2006).

20. Hart, G. W. \& Akimoto, Y. in Essentials of Glycobiology (eds Varki, A. et al.) (The Consortium of Glycobiology Editors, La Jolla, California, Cold Spring Harbor (NY), 2009).

21. Wells, L., Vosseller, K. \& Hart, G. W. Glycosylation of nucleocytoplasmic proteins: signal transduction and O-GlcNAc. Science 291, 2376-2378 (2001).

22. Hart, G. W., Housley, M. P. \& Slawson, C. Cycling of O-linked beta-N-acetylglucosamine on nucleocytoplasmic proteins. Nature 446, 1017-1022 (2007).

23. Love, D. C. \& Hanover, J. A. The hexosamine signaling pathway: deciphering the "O-GlcNAc code". SCi. STKE 2005, re13 (2005).

24. Hanover, J. A. Glycan-dependent signaling: O-linked N-acetylglucosamine. FASEB J. 15, 18651876 (2001).

25. Copeland, R. J., Han, G. \& Hart, G. W. O-GlcNAcomics - Revealing Roles of O-GlcNAcylation in Disease Mechanisms and Development of Potential Diagnostics. Proteomics Clin. Appl. (2013).

26. Butkinaree, C., Park, K. \& Hart, G. W. O-linked $\beta$-N-acetylglucosamine (O-GIcNAc): Extensive crosstalk with phosphorylation to regulate signaling and transcription in response to nutrients and stress. Biochimica et Biophysica Acta (BBA) - General Subjects 1800, 96-106 (2010).

27. Whelan, S. A., Lane, M. D. \& Hart, G. W. Regulation of the O-Linked ß-N-Acetylglucosamine Transferase by Insulin Signaling. Journal of Biological Chemistry 283, 21411-21417 (2008).

28. Song, M. et al. o-GlcNAc transferase is activated by CaMKIV-dependent phosphorylation under potassium chloride-induced depolarization in NG-108-15 cells. Cell. Signal. 20, 94-104 (2008).

29. Wang, Z., Pandey, A. \& Hart, G. W. Dynamic Interplay between O-Linked NAcetylglucosaminylation and Glycogen Synthase Kinase-3-dependent Phosphorylation. Molecular \& Cellular Proteomics 6, 1365-1379 (2007).

30. Wang, Z., Gucek, M. \& Hart, G. W. Cross-talk between GlcNAcylation and phosphorylation: Site-specific phosphorylation dynamics in response to globally elevated O-GIcNAc. Proceedings of the National Academy of Sciences 105, 13793-13798 (2008). 
31. Wang, Z. et al. Extensive crosstalk between O-GlcNAcylation and phosphorylation regulates cytokinesis. Sci. Signal. 3, ra2 (2010).

32. Hartweck, L. M., Scott, C. L. \& Olszewski, N. E. Two O-Linked N-Acetylglucosamine Transferase Genes of Arabidopsis thaliana L. Heynh. Have Overlapping Functions Necessary for Gamete and Seed Development. Genetics 161, 1279-1291 (2002).

33. Jacobsen, S. E. \& Olszewski, N. E. Mutations at the SPINDLY locus of Arabidopsis alter gibberellin signal transduction. Plant Cell 5, 887-896 (1993).

34. Hartweck, L. M. Gibberellin signaling. Planta 229, 1-13 (2008).

35. Thornton, T. M., Swain, S. M. \& Olszewski, N. E. Gibberellin signal transduction presents ...the SPY who O-GlcNAc'd me. Trends Plant Sci. 4, 424-428 (1999).

36. Olszewski, N. E., West, C. M., Sassi, S. O. \& Hartweck, L. M. O-GlcNAc protein modification in plants: Evolution and function. Biochimica et Biophysica Acta (BBA) - General Subjects 1800, 4956 (2010).

37. Kim, Y. C. et al. O-GIcNAcylation of the Plum pox virus capsid protein catalyzed by SECRET AGENT: characterization of O-GIcNAc sites by electron transfer dissociation mass spectrometry. Amino Acids 40, 869-876 (2011).

38. Perez Jde, J. et al. O-GIcNAc modification of the coat protein of the potyvirus Plum pox virus enhances viral infection. Virology 442, 122-131 (2013).

39. Hartweck, L. M., Genger, R. K., Grey, W. M. \& Olszewski, N. E. SECRET AGENT and SPINDLY have overlapping roles in the development of Arabidopsis thaliana L. Heyn. Journal of Experimental Botany 57, 865-875 (2006).

40. Koornneef, M. \& Veen, J. H. Induction and analysis of gibberellin sensitive mutants in Arabidopsis thaliana (L.) heynh. Theor. Appl. Genet. 58, 257-263 (1980).

41. Richards, D. E., King, K. E., Ait-Ali, T. \& Harberd, N. P. HOW GIBBERELLIN REGULATES PLANT GROWTH AND DEVELOPMENT: A Molecular Genetic Analysis of Gibberellin Signaling. Annu. Rev. Plant Physiol. Plant Mol. Biol. 52, 67-88 (2001).

42. Silverstone, A. L., Mak, P. Y. A., Martinez, E. C. \& Sun, T. The New RGA Locus Encodes a Negative Regulator of Gibberellin Response in Arabidopsis thaliana. Genetics 146, 1087-1099 (1997).

43. http://5e.plantphys.net/article.php?ch=\&id=382.

44. Daviere, J. M., de Lucas, M. \& Prat, S. Transcriptional factor interaction: a central step in DELLA function. Curr. Opin. Genet. Dev. 18, 295-303 (2008).

45. English, A. M. Development and Application of Methodologies to Facilitate Peptide Sequencing with Mass Spectrometry. ProQuest Dissertations and Theses (2011).

46. Hochuli, E., Döbeli, H. \& Schacher, A. New metal chelate adsorbent selective for proteins and peptides containing neighbouring histidine residues. Journal of Chromatography A 411, 177-184 (1987). 
47. Einhauer, A. \& Jungbauer, A. The FLAG ${ }^{\mathrm{TM}}$ peptide, a versatile fusion tag for the purification of recombinant proteins. J. Biochem. Biophys. Methods 49, 455-465 (2001).

48. Olsen, J. V., Ong, S. E. \& Mann, M. Trypsin cleaves exclusively C-terminal to arginine and lysine residues. Mol. Cell. Proteomics 3, 608-614 (2004).

49. Udeshi, N. D., Compton, P. D., Shabanowitz, J., Hunt, D. F. \& Rose, K. L. Methods for analyzing peptides and proteins on a chromatographic timescale by electron-transfer dissociation mass spectrometry. Nat. Protoc. 3, 1709-1717 (2008).

50. Sleno, L. The use of mass defect in modern mass spectrometry. J. Mass Spectrom. 47, 226236 (2012).

51. Gross, J. in 67-116 (Springer Berlin Heidelberg, 2011).

52. Zhang, G. et al. in (ed FenyÃ

53. Iribarne, J. V. \& Thomson, B. A. On the evaporation of small ions from charged droplets. J. Chem. Phys. 64, 2287-2294 (1976).

54. Wang, Z. et al. Enrichment and Site Mapping of O-Linked N-Acetylglucosamine by a Combination of Chemical/Enzymatic Tagging, Photochemical Cleavage, and Electron Transfer Dissociation Mass Spectrometry. Molecular \& Cellular Proteomics 9, 153-160 (2010).

55. Comer, F. I., Vosseller, K., Wells, L., Accavitti, M. A. \& Hart, G. W. Characterization of a Mouse Monoclonal Antibody Specific for O-Linked N-Acetylglucosamine. Anal. Biochem. 293, 169-177 (2001).

56. Vosseller, K. et al. O-Linked N-Acetylglucosamine Proteomics of Postsynaptic Density Preparations Using Lectin Weak Affinity Chromatography and Mass Spectrometry. Molecular \& Cellular Proteomics 5, 923-934 (2006).

57. Syka, J. E., Coon, J. J., Schroeder, M. J., Shabanowitz, J. \& Hunt, D. F. Peptide and protein sequence analysis by electron transfer dissociation mass spectrometry. Proc. Natl. Acad. Sci. U. S. A. $101,9528-9533$ (2004).

58. Zubarev, R. A. Reactions of polypeptide ions with electrons in the gas phase. Mass Spectrom. Rev. 22, 57-77 (2003).

59. Mohr, J. H., Swart, R. \& Huber, C. G. Morphology and efficiency of poly(styrene-codivinylbenzene)-based monolithic capillary columns for the separation of small and large molecules. Anal. Bioanal Chem. 400, 2391-2402 (2011).

60. Schulz, B. L., Packer, N. H. \& Karlsson, N. G. Small-scale analysis of O-linked oligosaccharides from glycoproteins and mucins separated by gel electrophoresis. Anal. Chem. 74, 6088-6097 (2002).

61. Simon, R., Enjalbert, Q., Biarc, J., Lemoine, J. \& Salvador, A. Evaluation of hydrophilic interaction chromatography (HILIC) versus $\mathrm{C} 18$ reversed-phase chromatography for targeted quantification of peptides by mass spectrometry. Journal of Chromatography A 1264, 31-39 (2012). 
62. Gilar, M. \& Jaworski, A. Retention behavior of peptides in hydrophilic-interaction chromatography. Journal of Chromatography A 1218, 8890-8896 (2011).

63. Krusemark, C. J., Ferguson, J. T., Wenger, C. D., Kelleher, N. L. \& Belshaw, P. J. Global amine and acid functional group modification of proteins. Anal. Chem. 80, 713-720 (2008).

64. Krusemark, C. J., Frey, B. L., Belshaw, P. J. \& Smith, L. M. Modifying the charge state distribution of proteins in electrospray ionization mass spectrometry by chemical derivatization. J. Am. Soc. Mass Spectrom. 20, 1617-1625 (2009).

65. Lundblad, R. L. in Protein Modification (C R C Press LLC, 1995).

66. Chou, P. Y. \& Fasman, G. D. Empirical Predictions of Protein Conformation. Annu. Rev. Biochem. 47, 251-276 (1978).

67. Shortreed, M. R. et al. lonizable isotopic labeling reagent for relative quantification of amine metabolites by mass spectrometry. Anal. Chem. 78, 6398-6403 (2006).

68. Hart, G. W. \& West, C. M. in Essentials of Glycobiology (eds Varki, A. et al.) (The Consortium of Glycobiology Editors, La Jolla, California, Cold Spring Harbor (NY), 2009).

69. Bork, P., Downing, A. K., Kieffer, B. \& Campbell, I. D. Structure and distribution of modules in extracellular proteins. Q. Rev. Biophys. 29, 119-167 (1996).

70. Hase, S. et al. A new trisaccharide sugar chain linked to a serine residue in bovine blood coagulation factors VII and IX. J. Biochem. 104, 867-868 (1988).

71. Bjoern, S. et al. Human plasma and recombinant factor VII. Characterization of Oglycosylations at serine residues 52 and 60 and effects of site-directed mutagenesis of serine 52 to alanine. J. Biol. Chem. 266, 11051-11057 (1991).

72. Harris, R. J., Ling, V. T. \& Spellman, M. W. O-linked fucose is present in the first epidermal growth factor domain of factor XII but not protein C. J. Biol. Chem. 267, 5102-5107 (1992).

73. Buko, A. M. et al. Characterization of a posttranslational fucosylation in the growth factor domain of urinary plasminogen activator. Proc. Natl. Acad. Sci. U. S. A. 88, 3992-3996 (1991).

74. Moloney, D. J. et al. Mammalian Notch1 is modified with two unusual forms of O-linked glycosylation found on epidermal growth factor-like modules. J. Biol. Chem. 275, 9604-9611 (2000).

75. Takeuchi, H. \& Haltiwanger, R. S. Role of glycosylation of Notch in development. Semin. Cell Dev. Biol. 21, 638-645 (2010).

76. Stanley, P. \& Okajima, T. in Current Topics in Developmental Biology 131-164 (Academic Press.

77. Matsuura, A. et al. O-linked N-acetylglucosamine is present on the extracellular domain of notch receptors. J. Biol. Chem. 283, 35486-35495 (2008).

78. Sakaidani, Y. et al. O-linked-N-acetylglucosamine modification of mammalian Notch receptors by an atypical O-GIcNAc transferase Eogt1. Biochem. Biophys. Res. Commun. 419, 1419 (2012). 
79. Li, W. et al. Statistical analysis of electron transfer dissociation pairwise fragmentation patterns. Anal. Chem. 83, 9540-9545 (2011).

80. Good, D. M., Wirtala, M., McAlister, G. C. \& Coon, J. J. Performance characteristics of electron transfer dissociation mass spectrometry. Mol. Cell. Proteomics 6, 1942-1951 (2007).

81. - SELECTIVE CLEAVAGE OF THE METHIONYL PEPTIDE BONDS IN RIBONUCLEASE WITH CYANOGEN BROMIDE1. - J. Am. Chem. Soc., - 1510.

82. Andreev, Y. A., Kozlov, S. A., Vassilevski, A. A. \& Grishin, E. V. Cyanogen bromide cleavage of proteins in salt and buffer solutions. Anal. Biochem. 407, 144-146 (2010).

83. - Capasso, S., - Mazzarella, L., - Sica, F., - Zagari, A. \& - Salvadori, S. - Spontaneous cyclization of the aspartic acid side chain to the succinimide derivative. - J. Chem. Soc. , Chem. Commun., 919.

84. Tetaz, T., Morrison, J. R., Andreou, J. \& Fidge, N. H. Relaxed specificity of endoproteinase Asp-N: this enzyme cleaves at peptide bonds $\mathrm{N}$-terminal to glutamate as well as aspartate and cysteic acid residues. Biochem. Int. 22, 561-566 (1990).

85. Nomura, T. et al. Purification, cDNA cloning, and expression of UDP-Gal: glucosylceramide beta-1,4-galactosyltransferase from rat brain. J. Biol. Chem. 273, 13570-13577 (1998). 


\section{Chapter 3: Site-specific phosphorylation of the DNA damage response mediator rad 9 by cyclin-dependent kinases regulates activation of checkpoint kinase 1}

\subsection{Introduction}

\subsubsection{DNA Damage Response}

Eukaryotes have the ability to monitor and react to DNA damage including nicks, gaps, double-strand breaks, and any alterations that block DNA replication. This is known as the DNA damage response (DDR) whereby cells regulate DNA replication, transcription, and repair in

response to DNA damage. ${ }^{1}$. Deficiencies in the DDR have negative implications for pathologies like cancer, because cells fail to induce senescence and apoptosis ${ }^{1,2}$.

The DDR is a signal transduction pathway regulated in large part by the posttranslational modification, phosphorylation. Protein kinases activated by DNA damage are therefore key components of the DDR. In all organisms studied, these DNA-damage-sensing kinases include members from the phosphatidylinositol 3-kinase-like (PIKK) family. In humans, two PIKKs critical to DDR are ATM and ATR. Much of what is currently known about the DDR is due to studies in Saccharomyces cerevisiae and Schizosaccharomyces pombe. ATM and ATR respectively correspond to Mec1p and Tel1p in S. cerevisiae, and Rad3 and Tel1 in S. pombe ${ }^{3}$. After PIKKs are activated by DNA-protein complexes formed in response to DNA-damage, they regulate various downstream proteins involved in the DDR including two important effector "checkpoint" kinases. These are Chk1 and Chk2 in humans or the orthologous spChk1 and spCds1 in S. pombe, and scChk1 and scRad53 in S. cerevisiae ${ }^{4}$. They contain highly conserved kinase domains but are structurally and functionally distinct with little overlap in their 
downstream targets. Chk1 is primarily activated downstream of ATR in response to DNA comprises that include stalled replication forks, DNA crosslinks, ultraviolet radiation, and ionizing radiation. Chk2 is primarily activated downstream of ATM as a result of DNA doublestrand breaks ${ }^{4}$.

Another key component to the DDR are DDR mediator/adaptor proteins that localize to sites of DNA damage and are typically phosphorylated by multiple kinases including PIKKs in response to it ${ }^{5}$. DDR mediator proteins aid PIKK-dependent activation of checkpoint kinases by participating in the assembly of DNA damage foci and facilitating protein-protein interactions at sites of DNA damage ${ }^{6,7}$. The prototypical DDR mediator and first checkpoint protein discovered was Rad9 in S. cerevisiae ${ }^{8}$.

\subsubsection{Rad9}

Rad9 is a $148 \mathrm{kDa}$ protein essential for the arrest of cell division triggered by DNA damage. It is related to S. pombe mediator Crb2, and human mediators 53BP1, MDC1, and BRCA1. This central DDR mediator is required in late $\mathrm{G} 1$, intra-S, and at the $\mathrm{G} 2 / \mathrm{M}$ transition for checkpoint delays ${ }^{9}$. It detects DNA damage and arrests cells in G2 until the damage is repaired, and it also plays additional key roles in DNA damage repair. ${ }^{8}$. Rad9 is recruited to sites of DNA damage either by interaction with two histone modifications or with mediator protein Dpb11, also an activator of Mec1 ${ }^{10}$. Once recruited, Rad9's SQ/TQ cluster domain (SCD) becomes phosphorylated by PIKKs to induce its oligomerization and activation ${ }^{11}$. This hyperphosphorylated Rad9 forms a 560kDa complex that recruits mediator Rad53 so it too may be phosphorylated by PIKKs. This in turn promotes trans autophosphorylation of Rad53 to fully activate itself ${ }^{12,13}$. Activated Rad53 is released by Rad9, so it may transmit the checkpoint signal throughout the nucleus. 


\subsubsection{Checkpoint Kinase 1 (Chk1) Activation by Rad9}

Chk1 is PIKK-dependently phosphorylated on its C-terminus, leading to cis autophosphorylation in the same region and activation ${ }^{14}$. The mechanism by which Rad9 interacts with and activates Chk1 is much less understood than its role in Rad53 activation. Rad9 has mainly been connected to DNA damage-induced Chk1 activation due to its role in providing a protein-binding scaffold at sites of DNA damage. The N-terminus of Rad9 has been identified as critical for phosphorylation and activation of Chk1 and has been appropriately termed the Chk1 activation domain (CAD), although the details of how the CAD works are poorly understood $^{15}$.

DDR mediators are often phosphorylated during the cell cycle even in the absence of DNA damage ${ }^{16,17}$. Rad9 contains 20 Ser/Thr-Pro cyclin-dependent kinase (CDK) consensus motifs (9 are the full Ser/Thr-Pro-X-Lys/Arg motif) ${ }^{18}$. CDKs regulate and time events in the eukaryotic cell cycle and their activation requires their association with cyclin subunits whose concentrations fluctuate according to a variety of cellular factors ${ }^{19}$. One $\mathrm{CDK}$, cdc28, regulates cell-cycle transitions in S. cerevisiae. Three cyclins, Cln1-3, operate in conjunction with cdc28 in G1. Entry into S phase and mitosis is controlled by cdc28's association with cyclins Clbs5-6 and Clbs1-4, respectively ${ }^{20}$. Evidence exists for the phosphorylation of Rad9 by cdc28 in vitro ${ }^{18}$, and mass spectrometric analyses have revealed 13 of the 20 CDK sites to be phosphorylated in vivo 21-23. Still, the biological function of CDK-dependent Rad9 phosphorylation remains largely uncharacterized.

This study sought to characterize phosphorylation of the Rad9 CAD by $\mathrm{Cdc} 28 / \mathrm{Clb}$ complexes in S, G2, and M phases by mass spectrometry, and in collaboration with the Lowndes Lab at NUI Galway, determine its role in Chk1 activation. We report that phosphorylation of 
CDK consensus sites in the Rad9 CAD regulate a DNA damage-independent interaction between Chk1 and Rad9, and regulate activation of Chk1-dependent signaling in response to DNA damage. CDK sites T125 and T143 were shown to be especially important for Chk1 activation by Rad9. Our model suggests that CDK-dependent phosphorylation of the Rad9 CAD during S, G2, and $\mathrm{M}$ phases is required for interaction between Chk1 and Rad9. This enables phosphorylation of both proteins by PIKK, which leads to Chk1 activation and its subsequent exit from the site of DNA damage so it may perform its appropriate nuclear signaling ${ }^{24}$.

\subsection{Materials and Methods}

\subsubsection{Molecular biology methods}

Materials and methods used for yeast strains, cell cycle arrest and checkpoint experiments, antibodies, in vitro kinase assays, yeast two-hybrid experiments, yeast native extracts and immunoprecipitation, peptide pull-downs, and SPOT synthesis of peptides and array experiments are described in ${ }^{24}$.

\subsubsection{Rad9 purification}

Cell cycle-modified (C-Rad9) Rad9-GFP-FLAG was purified by double immunoaffinity purification from whole-cell extracts, prepared from $10 \mathrm{~L}$ of asynchronously growing, $\mathrm{G} 1 \mathrm{or}$

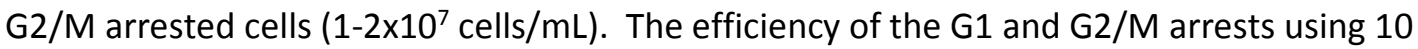
$\mu \mathrm{g} / \mathrm{ml} \alpha$-factor and $20 \mu \mathrm{g} / \mathrm{ml}$ nocodazole, respectively, was verified by microscopy. Approximately $1.5 \mathrm{~g}$ of total protein was obtained for each crude cell extract. Extracts were precleared with $200 \mu \mathrm{L}$ of packed protein G beads (GE Healthcare) prior to incubation with $200 \mu \mathrm{L}$ of packed FLAG M2-affinity beads (Sigma-Aldrich) for $2 \mathrm{~h}$ at $4^{\circ} \mathrm{C}$. Four successive elutions were performed using $200 \mu \mathrm{L}$ of $3 x F L A G$ peptide (100 $\mu \mathrm{g} / \mathrm{mL}$, Sigma-Aldrich) and Rad9-GFP-FLAG- 
containing fractions were identified by western blotting. $20 \mu \mathrm{L}$ of GFP-Trap agarose beads

(Chromotek) were added to the pooled Rad9-GFP-FLAG containing fractions. Batch binding was allowed to occur for $2 \mathrm{~h}$ at $4^{\circ} \mathrm{C}$ with gentle agitation. The beads were washed 3 times with wash buffer (150 mM KAc, 50 mM HEPES pH 7.5) and once with ultra-pure water. Washed beads were resuspended in an equal volume of $0.1 \%$ glacial acetic acid. The presence of purified Rad9GFP-FLAG was verified by silver stain gel on which $1 \mu \mathrm{L}$ of the final beads was loaded.

\subsubsection{Mass Spectrometric Analysis of Rad9-GFP-FLAG}

Rad9-GFP-FLAG purified from asynchronous, G1- or G2/M-arrested cells was reduced and carbamidomethylated on GFP beads at room temperature using dithiothreitol (Sigma Aldrich, St. Louis, MO) and iodoacetamide (Sigma Aldrich), respectively, then subjected to proteolytic digestion (1: 20 enzyme to substrate) using endoproteinase Lys-C (Roche, Penzberg, Germany) using methods similar to those previously reported ${ }^{25}$. Half of each sample was subjected to sub-digestion using trypsin (Promega, Madison, WI) and a quarter of the Lys-C digested asynchronous sample was subdigested with endoproteinase Asp-N (Roche, Penzberg, Germany), at the same ratios as above. Prior to mass spectrometric analysis, each digest was dried down and resuspended in $0.1 \%$ acetic acid in water. For each analysis, a 1 pmol fraction was pressure loaded onto a $360 \mu \mathrm{m}$ o.d. $\times 75 \mu \mathrm{m}$ i.d. fused silica capillary precolumn packed with $5 \mathrm{~cm}$ of $\mathrm{C} 18$

reverse-phase resin (5-20 $\mu$ m diameter, $120 \AA ̊$ pore size, YMC Co., Ltd., Kyoto, Japan). Following a 15 min desalting rinse using $0.1 \mathrm{M}$ acetic acid, the precolumn was connected to a $360 \mu \mathrm{m}$ o.d. $\times 50 \mu \mathrm{m}$ i.d. analytical column packed with $6-8 \mathrm{~cm}$ of $\mathrm{C} 18$ resin $(5 \mu \mathrm{m}$ diameter, $120 \AA$ A pore size, YMC Co., Ltd.) and equipped with an electrospray emitter tip as previously described ${ }^{26}$. Endoproteinase Lys-C or trypsin generated peptides were then gradient eluted into the mass spectrometer using electrospray ionization at a flow rate of $60 \mathrm{~nL} / \mathrm{min}$ using an $\mathrm{LC}$ gradient 
previously described ${ }^{27}$. Mass analysis was completed using an acquisition method consisting of one high resolution MS1 scan (resolving power of 50,000 (FT Ultra) or 60,000 (Orbitrap) at $\mathrm{m} / \mathrm{z}$ 400) acquired in the FT-ICR or Orbitrap followed by 5 data dependent MS/MS scans using collision-activated dissociation (CAD) and electron transfer dissociation (ETD) acquired in the ion trap of the LTQ-FT Ultra or LTQ-Orbitrap hybrid instrument (Thermo Fisher Scientific, Bremen, Germany). Data dependent parameters included a repeat count of 3, repeat duration of $30 \mathrm{~s}$, and exclusion list duration of $30 \mathrm{~s}$. MS/MS parameters for ETD scans included $35 \mathrm{~ms}$ reaction time, $3 \mathrm{~m} / \mathrm{z}$ precursor isolation window, charge state rejection "on" for +1 and unassigned charge state precursor ions, $5 \times 10^{5}$ FTMS (Orbitrap) or $1 \times 10^{6}$ (FT Ultra) full automated gain control target, $1 \times 10^{4} \mathrm{ITMS}^{\mathrm{n}}$ automated gain control target, and $2 \times 10^{5}$ reagent target with azulene as the electron transfer reagent.

\subsection{Results and Discussion}

\subsubsection{Rad9-GFP-FLAG Purification}

Rad9-GFP-FLAG was expressed in three separate samples of $S$. cerevisiae in the absence of DNA damage. In the first, the cells were allowed to remain asynchronous. In the second and third, the cells were arrested at cell cycle stages $\mathrm{G} 1$ and G2, respectively. All three were tandemimmunopurified to generate highly enriched protein amenable for the detection of posttranslational modifications by mass spectrometry. Samples were firstly bound to anti-FLAG M2 affinity beads, washed, and eluted with 3xFLAG peptide. These Rad9-enriched samples were then bound to GFP-trap A beads for a second round of purification, washed, and resuspended in $0.01 \%$ acetic acid $(\mathrm{pH} 4)$ until on-beads digestion. This yielded several pmols of purified Rad9 protein from each sample (Figure 3.1). 

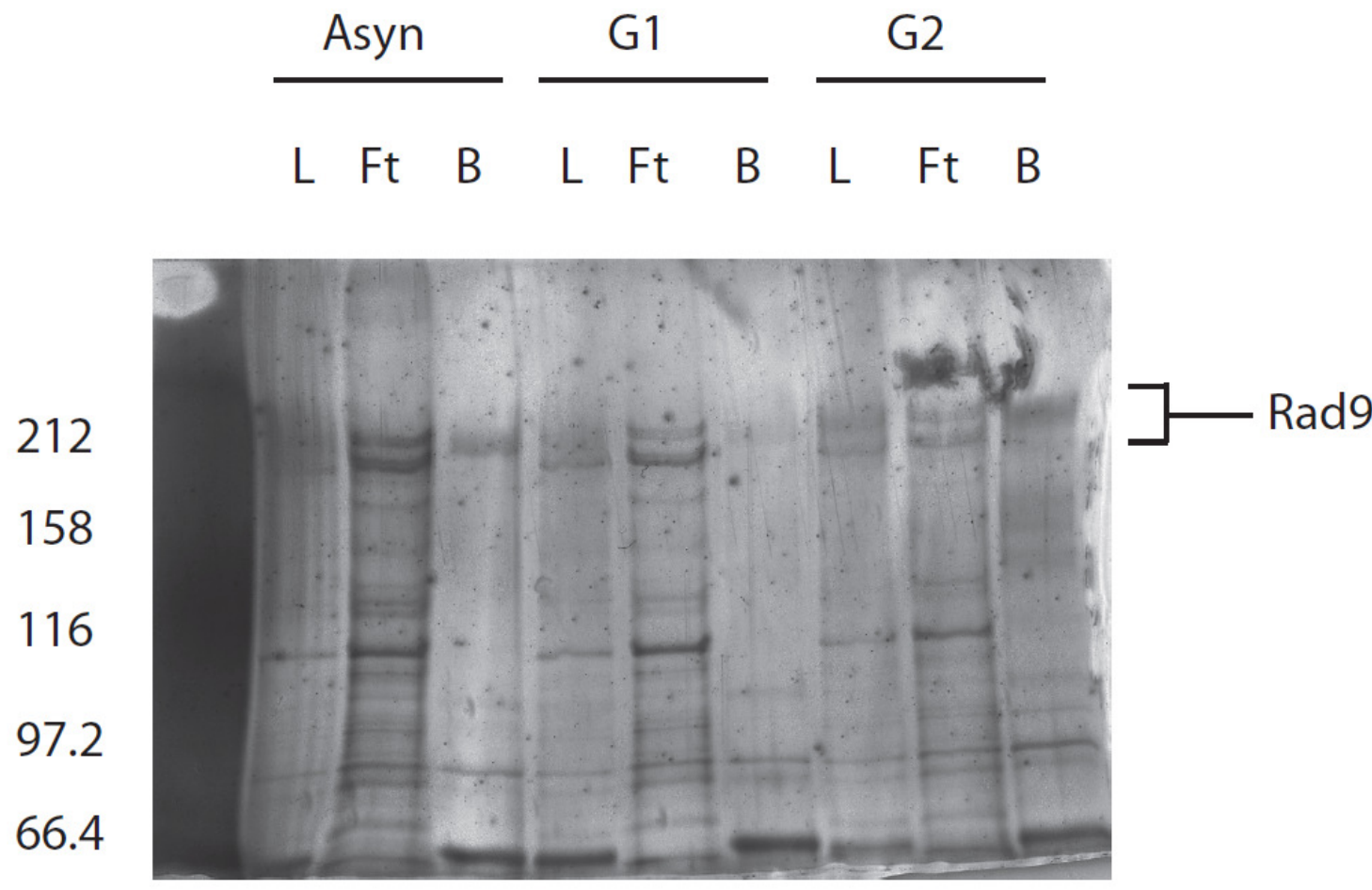

Figure 3.1 - Tandem purified Rad9-GFP-FLAG visualized by Silver stain on a 6\% acrylamide SDS-PAGE gel. L $-2.5 \%$ of Rad9 purified by FLAG IP. Ft $-2.5 \%$ of unbound flowthrough from FLAG IP. B-2.5\% of bound sample from GFP-IP.

\subsubsection{In silico Rad9 Digestion}

Simulated digestions of Rad9 were performed to determine candidate peptides that would allow for the detection of all putative CDK sites in the Chk1 activation domain. Two separate theoretical digests using Endoproteinase Lys-C and trypsin generated peptides that met this goal, and covered $>90 \%$ of Rad9 as a whole (Tables 3.1 \& 3.2).

\begin{tabular}{|c|c|c|c|c|}
\hline Mass & Position & Peptide & CDK Site & Comments \\
\hline 1075.5 & $1-9$ & MSGQLVQWK & & + \\
\hline 1287.7 & $10-14$ & SSPDRVTQSAIK & S11 & + \\
\hline 2734.3 & $22-46$ & EALHSPLADGDMNEMNVPVD PLENK & S26 & + \\
\hline 1257.7 & $47-58$ & VNSTNIIEGSPK & & + \\
\hline 738.4 & $59-65$ & ANPNPVK & S56 & $?$ \\
\hline 1243.6 & $66-75$ & FMNTSEIFQK & & + \\
\hline
\end{tabular}


Chapter 3

Phosphorylation Analysis of Rad9

\begin{tabular}{|c|c|c|c|c|}
\hline 5283.6 & $76-121$ & $\begin{array}{c}\text { SLGLLDESPRHDDELNIEVG } \\
\text { DNDRPNANILHNERTPDLDR IANFFK }\end{array}$ & S83, T110 & ? \\
\hline 758.4 & $122-128$ & SNRTPGK & & ? \\
\hline 716.4 & $129-134$ & ENLLTK & & - \\
\hline 1794.9 & $135-149$ & YQSSDLEDTPLMLRK & T143 & + \\
\hline 1534.7 & $151-163$ & MTFQTPTDPLEQK & T155 & + \\
\hline 5552.4 & $170-220$ & $\begin{array}{c}\text { SDTGFCYYGEQNDGEENASL } \\
\text { EVTEADATFVQMAERSADNY DCALEGIVTPK }\end{array}$ & T218 & ? \\
\hline 590.3 & $224-228$ & DELSK & & - \\
\hline 1233.6 & $229-239$ & SGGMQDERVQK & & + \\
\hline 1969.9 & $240-257$ & TQIMISAESPNSISSYDK & S248 & + \\
\hline 1429.8 & $260-272$ & ITGNGRTTRNVNK & & + \\
\hline 1704.8 & $273-287$ & VFNNNEDNIGAIEEK & & + \\
\hline 3581.6 & $294-323$ & SENYSSDDLRERNNQIIQSN ESEEINELEK & & + \\
\hline 5264.4 & $324-371$ & $\begin{array}{c}\text { NLNVSGRENDVNNLDIDINS } \\
\text { AVSGTPSRNNAEEEMYSSES VNNREPSK }\end{array}$ & T348 & ? \\
\hline 998.5 & $373-379$ & WIFRYSK & & ? \\
\hline 3826.9 & $382-415$ & TENNSNRSTQIVNNPRTQEM PLDSISIDTQPLSK & & ? \\
\hline 2937.5 & $416-442$ & SFNTETNNELETQIIVSSLS QGISAQK & & + \\
\hline 1528.8 & $443-456$ & GPVFHSTGQTEEIK & & + \\
\hline 4511.3 & $457-497$ & $\begin{array}{l}\text { TQIINSPEQNALNATFETPV } \\
\text { TLSRINFEPILEVPETSSPS K }\end{array}$ & $\begin{array}{l}\text { S462, T474, } \\
\text { S494 }\end{array}$ & ? \\
\hline 579.3 & $498-502$ & NTMSK & & - \\
\hline
\end{tabular}


Chapter 3

Phosphorylation Analysis of Rad9

\begin{tabular}{|c|c|c|c|c|}
\hline 925.5 & $503-511$ & PSNSSPIPK & S507 & ? \\
\hline 4954.3 & $514-557$ & $\begin{array}{c}\text { DTFNIHEREVETNNVFSNDI } \\
\text { QNSSNAATRDDIIIAGSSDF NEQK }\end{array}$ & & ? \\
\hline 1534.8 & $558-570$ & EITDRIYLQLSGK & & + \\
\hline 2338.0 & $571-591$ & QISDSGSDETERMSPNELDT K & & + \\
\hline 3216.5 & $593-620$ & ESTIMSEVELTQELPEVEEQ QDLQTSPK & & + \\
\hline 1431.8 & $622-633$ & LVVEEETLMEIK & & + \\
\hline 1239.6 & $637-647$ & GNSLQLHDDNK & & + \\
\hline 694.3 & $648-653$ & ECNSDK & & - \\
\hline 1869.9 & $654-670$ & QDGTESLDVALIEHESK & & + \\
\hline 875.4 & $671-678$ & GQSSELQK & & ? \\
\hline 2544.3 & $679-699$ & NLMQLFPSESQEIIQNRRTI K & & + \\
\hline 586.4 & $700-703$ & RRQK & & - \\
\hline 1748.8 & $704-718$ & DTIEIGEEEENRSTK & & + \\
\hline 532.3 & $719-723$ & TSPTK & S720 & - \\
\hline 1188.6 & $727-737$ & RNSDLDAASIK & & + \\
\hline 1849.9 & $738-755$ & REPSCSITIQTGETGSGK & & + \\
\hline 2287.1 & $759-778$ & EQSYVFPEGIRTADNSFLSK & S584 & + \\
\hline 2235.0 & $779-796$ & DDIIFGNAVWCQYTWNYK & S618 & + \\
\hline 3264.6 & $797-824$ & FYPGILLEVDTNQDGCWIYF ETGRSLTK & & + \\
\hline 5684.8 & $825-873$ & $\begin{array}{c}\text { DEDIYYLDIRIGDAVTFDGN } \\
\text { EYVVVGLECRSHDLNIIRCI RGYDTVHLK }\end{array}$ & & ? \\
\hline 758.4 & $876-883$ & NASGLLGK & & ? \\
\hline 629.4 & $884-888$ & RTLIK & & - \\
\hline 1518.8 & $889-902$ & ALSSISLDLSEWAK & & + \\
\hline 972.5 & $906-913$ & IILEDNEK & & ? \\
\hline 1857.0 & $916-930$ & GDAYRYLRHPIRGRK & & + \\
\hline
\end{tabular}


Chapter 3

Phosphorylation Analysis of Rad9

140

\begin{tabular}{|c|c|c|c|c|}
\hline 975.5 & 931-939 & SMTNVLSPK & S937 & + \\
\hline 743.3 & $941-946$ & HTDDEK & & - \\
\hline 2208.0 & $947-965$ & DINTHTEVYNNEIESSSEK & & + \\
\hline 3159.6 & $\begin{array}{l}972- \\
1001\end{array}$ & DSRDALAEHAGAPSLLFSSG EIRTGNVFDK & & ? \\
\hline 4858.5 & $\begin{array}{l}1002- \\
1044\end{array}$ & $\begin{array}{c}\text { CIFVLTSLFENREELRQTIE } \\
\text { SQGGTVIESGFSTLFNFTHP LAK }\end{array}$ & & ? \\
\hline 559.3 & $\begin{array}{l}1045- \\
1049\end{array}$ & SLVNK & & - \\
\hline 1342.7 & $\begin{array}{l}1050- \\
1061\end{array}$ & GNTDNIRELALK & & + \\
\hline 516.3 & $\begin{array}{l}1062- \\
1065\end{array}$ & LAWK & & - \\
\hline 1820.9 & $\begin{array}{l}1066- \\
1081\end{array}$ & PHSLFADCRFACLITK & & + \\
\hline 908.6 & $\begin{array}{l}1082- \\
1088\end{array}$ & RHLRSLK & & ? \\
\hline 1827.0 & $\begin{array}{l}1089- \\
1103\end{array}$ & YLETLALGWPTLHWK & & + \\
\hline 909.5 & $\begin{array}{l}1104- \\
1111\end{array}$ & FISACIEK & & ? \\
\hline 3114.7 & $\begin{array}{l}1113- \\
1139\end{array}$ & RIVPHLIYQYLLPSGESFRL SLDSPSK & & + \\
\hline 2906.4 & $\begin{array}{l}1145- \\
1169\end{array}$ & SNNIFSFYTQFLRGSNLRDQ ICGVK & & + \\
\hline 2383.2 & $\begin{array}{l}1171- \\
1190\end{array}$ & MLNDYIVIVWGRSELDSFVK & & + \\
\hline 4999.6 & $\begin{array}{l}1191- \\
1236\end{array}$ & $\begin{array}{c}\text { FAFACLSAGRMLTIDLPNID } \\
\text { VDDTEPLLNALDSLVPRIGS ELSNRK }\end{array}$ & & ? \\
\hline 1281.6 & $\begin{array}{l}1239- \\
1249\end{array}$ & FLIYANENNGK & & + \\
\hline 1454.9 & $\begin{array}{l}1254- \\
1265\end{array}$ & LLERLRSQISLK & & + \\
\hline 1284.6 & $\begin{array}{c}1269- \\
1278\end{array}$ & FNYIFHTESK & & + \\
\hline
\end{tabular}




\begin{tabular}{|c|c|c|c|c|}
\hline \multirow{4}{*}{4657.3} & $1279-$ & EWLIQTIINEDTGFHDDITD & & \\
& 1318 & NDIYNTISEVR(RIPGLINSK) & & $?$ \\
& & & & \\
\hline
\end{tabular}

Table 3.1 - In silico Lys-C digestion of Rad9 displaying all >500 Da peptides. Residues belonging to the C-terminal tag linker are indicated in parentheses. Peptide masses (Da), position numbers, and putative CDK sites are indicated. The comments section refers to peptides predicted to be detected (+) or not detected (-), with (?) indicating uncertainty of detection.

\begin{tabular}{|c|c|c|c|c|}
\hline Mass & Position & Peptide & CDK Site & Comments \\
\hline 1075.5 & $1-9$ & MSGQLVQWK & & + \\
\hline 560.3 & $10-14$ & SSPDR & S11 & - \\
\hline 745.4 & $15-21$ & VTQSAIK & & $?$ \\
\hline 2734.3 & $22-46$ & EALHSPLADGDMNEMNVPVD PLENK & S26 & + \\
\hline 1257.7 & $47-58$ & VNSTNIIEGSPK & S56 & + \\
\hline 738.4 & $59-65$ & ANPNPVK & & + \\
\hline 1243.6 & $66-75$ & FMNTSEIFQK & & + \\
\hline 1085.6 & $76-85$ & SLGLLDESPR & S83 & + \\
\hline 2798.3 & $86-109$ & HDDELNIEVGDNDRPNANIL HNER & & + \\
\hline 715.4 & $110-115$ & TPDLDR & T110 & $?$ \\
\hline 738.4 & $116-121$ & IANFFK & & $?$ \\
\hline 716.4 & $129-134$ & ENLLTK & & $?$ \\
\hline 1666.8 & $135-148$ & YQSSDLEDTPLMLR & T143 & + \\
\hline 1534.7 & $151-163$ & MTFQTPTDPLEQK & T155 & + \\
\hline 3875.6 & $170-204$ & $\begin{array}{l}\text { SDTGFCYYGEQNDGEENASL } \\
\text { EVTEADATFVQMAER }\end{array}$ & & ? \\
\hline 1694.8 & $205-220$ & SADNYDCALEGIVTPK & $\mathrm{T} 218$ & + \\
\hline 590.3 & $224-228$ & DELSK & & - \\
\hline 878.4 & $229-236$ & SGGMQDER & & + \\
\hline 1969.9 & $240-257$ & TQIMISAESPNSISSYDK & S248 & + \\
\hline 616.3 & $260-265$ & ITGNGR & & $?$ \\
\hline 1704.8 & $273-287$ & VFNNNEDNIGAIEEK & & + \\
\hline 1184.5 & 294-303 & SENYSSDDLR & & + \\
\hline
\end{tabular}




\begin{tabular}{|c|c|c|c|c|}
\hline 2130.0 & $306-323$ & NNQIIQSNESEEINELEK & & + \\
\hline 758.4 & $324-330$ & NLNVSGR & & ? \\
\hline 2229.1 & $331-351$ & ENDVNNLDIDINSAVSGTPS R & T348 & + \\
\hline 1871.8 & $352-367$ & NNAEEEMYSSESVNNR & & + \\
\hline 620.3 & $373-376$ & WIFR & & - \\
\hline 833.4 & $382-388$ & TENNSNR & & ? \\
\hline 1027.5 & $389-397$ & STQIVNNPR & & + \\
\hline 2002.0 & $398-415$ & TQEMPLDSISIDTQPLSK & & + \\
\hline 2937.5 & $416-442$ & SFNTETNNELETQIIVSSLS QGISAQK & & + \\
\hline 1528.8 & $443-456$ & GPVFHSTGQTEEIK & & + \\
\hline 2643.4 & $457-480$ & TQIINSPEQNALNATFETPV TLSR & S462, T474 & + \\
\hline 1886.0 & $481-497$ & INFEPILEVPETSSPSK & S494 & + \\
\hline 1486.7 & $498-511$ & NTMSKPSNSSPIPK & S507 & + \\
\hline 1030.5 & $514-521$ & DTFNIHER & & ? \\
\hline 2309.1 & $522-542$ & EVETNNVFSNDIQNSSNAAT R & & + \\
\hline 1650.8 & $543-557$ & DDIIIAGSSDFNEQK & & + \\
\hline 632.3 & $558-562$ & EITDR & & - \\
\hline 920.5 & $563-570$ & IYLQLSGK & & ? \\
\hline 1322.6 & $571-582$ & QISDSGSDETER & & + \\
\hline 1033.5 & 583-591 & MSPNELDTK & S584 & + \\
\hline 3216.5 & $593-620$ & ESTIMSEVELTQELPEVEEQ QDLQTSPK & S618 & + \\
\hline 1431.8 & $622-633$ & LVVEEETLMEIK & & + \\
\hline 1239.6 & 637-647 & GNSLQLHDDNK & & + \\
\hline 694.3 & $648-653$ & ECNSDK & & - \\
\hline 1869.9 & $654-670$ & QDGTESLDVALIEHESK & & + \\
\hline 875.4 & $671-678$ & GQSSELQK & & ? \\
\hline 2046.0 & $679-695$ & NLMQLFPSESQEIIQNR & & + \\
\hline 1432.6 & 704-715 & DTIEIGEEEENR & & + \\
\hline 532.3 & $719-723$ & TSPTK & S720 & - \\
\hline 1032.5 & $728-737$ & NSDLDAASIK & & + \\
\hline
\end{tabular}


Chapter 3

Phosphorylation Analysis of Rad9

\begin{tabular}{|c|c|c|c|c|}
\hline 1693.8 & $739-755$ & EPSCSITIQTGETGSGK & & + \\
\hline 1323.6 & 759-769 & EQSYVFPEGIR & & + \\
\hline 981.5 & 770-778 & TADNSFLSK & & + \\
\hline 2235.0 & 779-796 & DDIIFGNAVWCQYTWNYK & & + \\
\hline 2835.3 & $797-820$ & FYPGILLEVDTNQDGCWIYF ETGR & & + \\
\hline 1313.6 & $825-834$ & DEDIYYLDIR & & + \\
\hline 2155.0 & $835-854$ & IGDAVTFDGNEYVVVGLECR & & + \\
\hline 966.5 & $855-862$ & SHDLNIIR & & ? \\
\hline 931.5 & $866-873$ & GYDTVHLK & & ? \\
\hline 758.4 & $876-883$ & NASGLLGK & & ? \\
\hline 1518.8 & $889-902$ & ALSSISLDLSEWAK & & + \\
\hline 972.5 & $906-913$ & IILEDNEK & & ? \\
\hline 580.3 & $916-920$ & GDAYR & & - \\
\hline 521.3 & $924-927$ & HPIR & & - \\
\hline 975.5 & $931-939$ & SMTNVLSPK & S937 & + \\
\hline 743.3 & $941-946$ & HTDDEK & & - \\
\hline 2208.0 & $947-965$ & DINTHTEVYNNEIESSSEK & & + \\
\hline 2040.0 & $975-994$ & DALAEHAGAPSLLFSSGEIR & & + \\
\hline 779.4 & 995-1001 & TGNVFDK & & ? \\
\hline 1440.7 & $\begin{array}{c}1002- \\
1013\end{array}$ & CIFVLTSLFENR & & + \\
\hline 545.3 & $\begin{array}{c}1014- \\
1017\end{array}$ & EELR & & - \\
\hline 2908.5 & $\begin{array}{c}1018- \\
1044\end{array}$ & QTIESQGGTVIESGFSTLFN FTHPLAK & & + \\
\hline 559.3 & $\begin{array}{c}1045- \\
1049\end{array}$ & SLVNK & & - \\
\hline 788.4 & $\begin{array}{c}1050- \\
1056\end{array}$ & GNTDNIR & & ? \\
\hline 572.4 & $\begin{array}{c}1057- \\
1061\end{array}$ & ELALK & & - \\
\hline 1542.8 & $\begin{array}{c}1062- \\
1074\end{array}$ & LAWKPHSLFADCR & & + \\
\hline 794.4 & $\begin{array}{c}1075- \\
1081\end{array}$ & FACLITK & & ? \\
\hline
\end{tabular}


Chapter 3

Phosphorylation Analysis of Rad9

\begin{tabular}{|c|c|c|c|c|}
\hline 1827.0 & $\begin{array}{c}1089- \\
1103\end{array}$ & YLETLALGWPTLHWK & & + \\
\hline 909.5 & $\begin{array}{c}1104- \\
1111\end{array}$ & FISACIEK & & ? \\
\hline 2131.1 & $\begin{array}{c}1114- \\
1131\end{array}$ & IVPHLIYQYLLPSGESFR & & + \\
\hline 845.4 & $\begin{array}{l}1132- \\
1139\end{array}$ & LSLDSPSK & S1136 & ? \\
\hline 1635.8 & $\begin{array}{l}1145- \\
1157\end{array}$ & SNNIFSFYTQFLR & & + \\
\hline 545.3 & $\begin{array}{c}1158- \\
1162\end{array}$ & GSNLR & & - \\
\hline 761.4 & $\begin{array}{c}1163- \\
1169\end{array}$ & DQICGVK & & ? \\
\hline 1477.8 & $\begin{array}{l}1171- \\
1182\end{array}$ & MLNDYIVIVWGR & & + \\
\hline 923.5 & $\begin{array}{c}1183- \\
1190\end{array}$ & SELDSFVK & & ? \\
\hline 1041.5 & $\begin{array}{c}1191- \\
1200\end{array}$ & FAFACLSAGR & & + \\
\hline 2991.5 & $\begin{array}{c}1201- \\
1227\end{array}$ & MLTIDLPNIDVDDTEPLLNA LDSLVPR & & + \\
\hline 874.5 & $\begin{array}{c}1228- \\
1235\end{array}$ & IGSELSNR & & ? \\
\hline 1281.6 & $\begin{array}{l}1239- \\
1249\end{array}$ & FLIYANENNGK & & + \\
\hline 529.3 & $\begin{array}{l}1254- \\
1257\end{array}$ & LLER & & - \\
\hline 674.4 & $\begin{array}{c}1260- \\
1265\end{array}$ & SQISLK & & - \\
\hline 1284.6 & $\begin{array}{c}1269- \\
1278\end{array}$ & FNYIFHTESK & & + \\
\hline 3678.7 & $\begin{array}{c}1279- \\
1309\end{array}$ & EWLIQTIINEDTGFHDDITDNDIYNTISEVR & & + \\
\hline
\end{tabular}

Table 3.2 - In silico Trypsin digestion of Rad9 displaying all >500 Da peptides. Residues belonging to the C-terminal tag linker are indicated in parentheses. Peptide masses (Da), position numbers, and putative CDK sites are indicated. The comments section refers to peptides predicted to be detected (+) or not detected (-), with (?) indicating uncertainty of detection. 


\subsubsection{Analysis of Rad9 by LCMS}

Using Lys-C and tryptic Rad9 digests, 70\% of the Rad9's 1309 residues were detected in each cell cycle type, including 19 of the 20 putative CDK phosphorylation sites (Figure 3.2).

\section{Rad9 Sequence Coverage}

MSGQLVQWKS SPDRVTQSAI KEALHSPLAD GDMNEMNVPV DPLENKVNST NIIEGSPKAN 60

PNPVKFMNTS EIFQKSLGLL DESPRHDDEL NIEVGDNDRP NANILHNERT PDLDRIANFF 120

KSNRTPGKEN LLTKYQSSDL EDTPLMLRKK MTFOTPTDPL EQKTFKKLKS DTGFCYYGEQ 180

NDGEENASLE VTEADATFVQ MAERSADNYD CALEGIVTPK RYKDELSKSG GMQDERVQKT 240

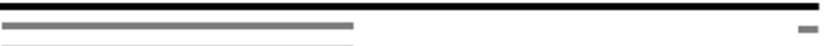

QIMISAESPN SISSYDKNKI TGNGRTTRNV NKVFNNNEDN IGAIEEKNPV KKKSENYSSD 300

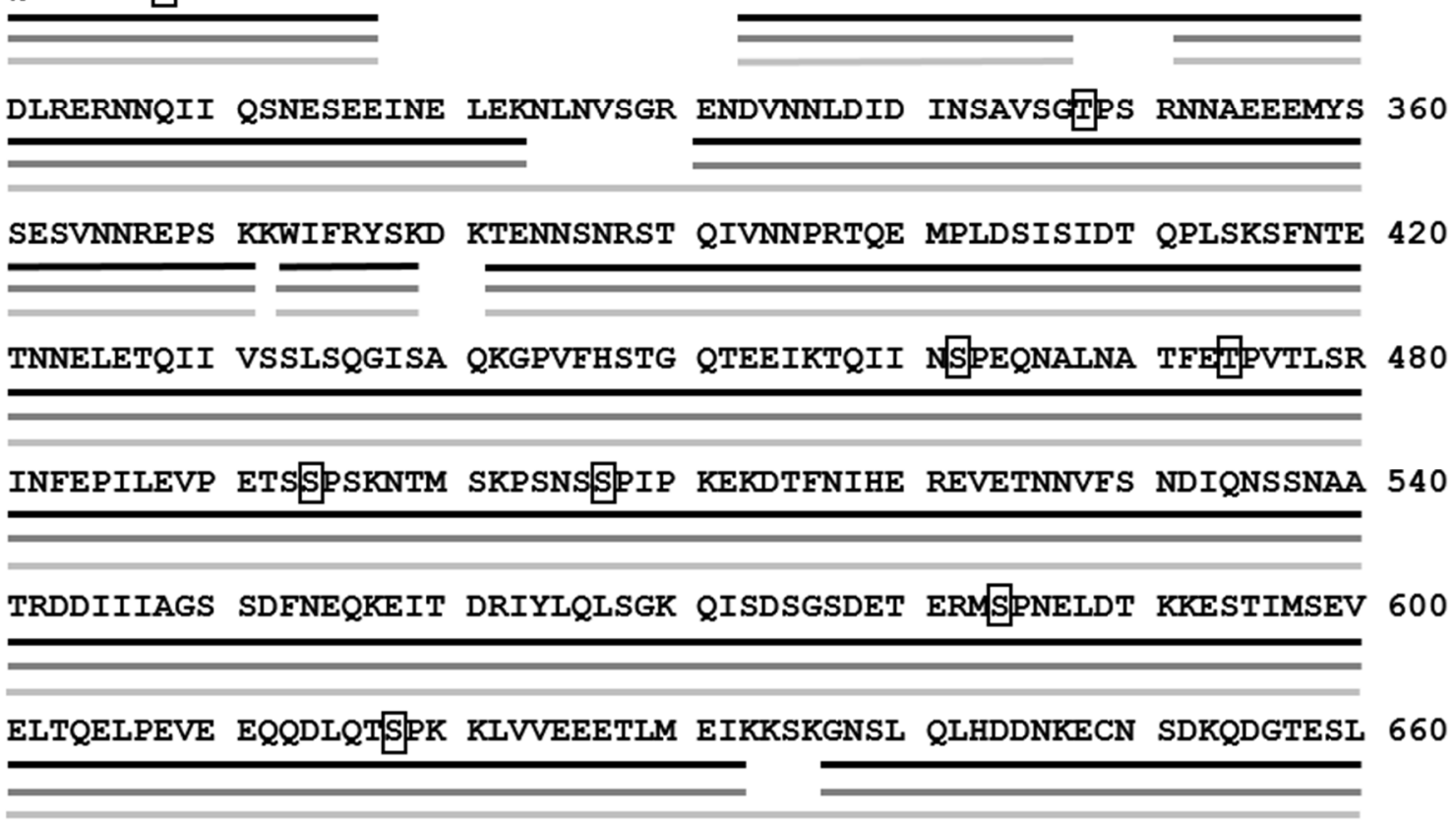


DVALIEHESK GQSSELQKNL MQLFPSESQE IIQNRRTIKR RQKDTIEIGE EEENRSTKIS 720

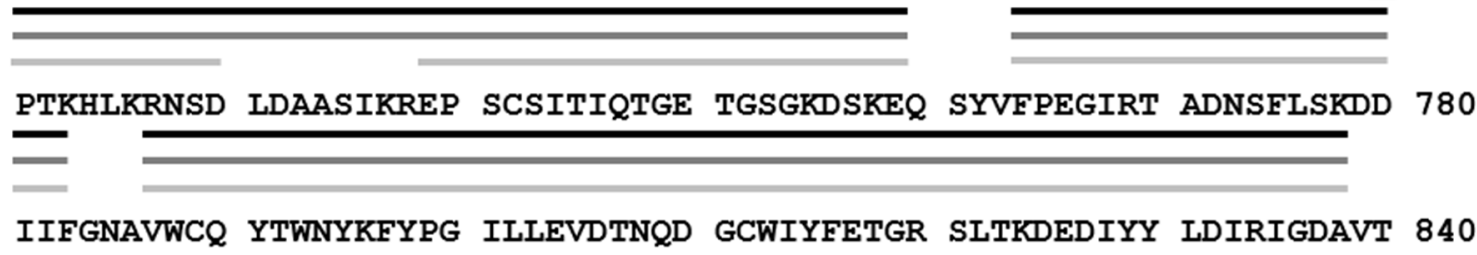

FDGNEYVVVG LECRSHDLNI IRCIRGYDTV HLKKKNASGL LGKRTLIKAL SSISLDLSEW 900

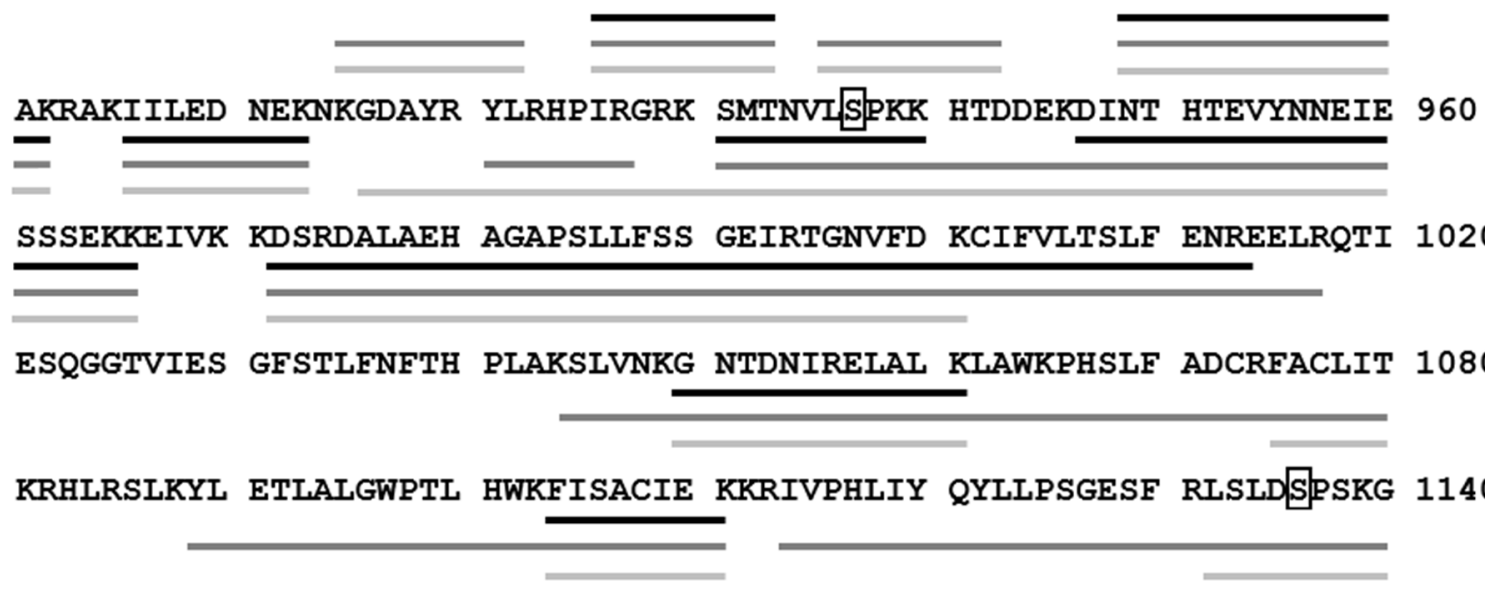

GIIKSNNIFS FYTQFLRGSN LRDQICGVKK MLNDYIVIVW GRSELDSFVK FAFACLSAGR 1200

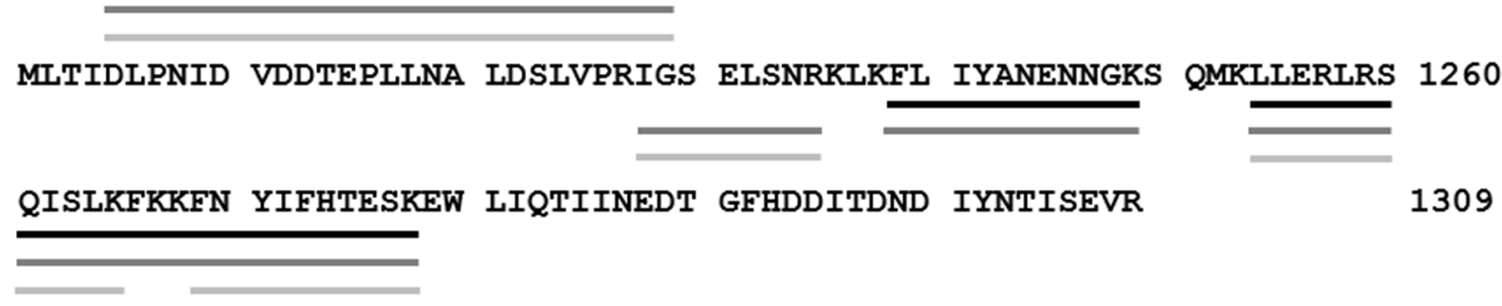

Figure 3.2 - Sequence coverage of trypsin and Lys-C-digested Rad9 from asynchronous (black), G1- (dark gray), and G2-arrested (light gray) cells. Asynchronous coverage includes subdigestion of Lys-C with Asp-N. Putative CDK phosphorylation sites are boxed.

The remaining CDK site, T110 located in the CAD, was detected by subdigestion of Lys-C-

generated peptides with Asp-N. CDK site T110 was not detectable in trypsin or Lys-C digests, because the peptides containing it did not sufficiently elute (Lys-C) or bind to the C18 (trypsin). The asynchronous Rad9 peptide SLGLLDESPRHDDELNIEVGDNDRPNANILHNERTPDLDRIANFFK, generated from Lys-C, was subdigested with Asp- $\mathrm{N}$ to produce the detectable peptide, DRPNANILHNERTP. All 20 CDK-site-containing peptides were found to be phosphorylated in one 
Chapter 3

Phosphorylation Analysis of Rad9

147

or more cell cycle forms of Rad9 and phosphorylation was unambiguously localized to 19 of

these 20 sites. It is likely that the undetermined site, T348, was also phosphorylated because a

phosphate was detected on the C-terminal half of peptide ENDVNNLDIDIN(SAVSGTPS)R as

indicated in parentheses, and T348 is located in this region. With the combination of Lys-C, Lys-

C-Asp- $\mathrm{N}$, and tryptic digests, peptides containing all 20 CDK sites were detected and found to be phosphorylated (Table 3.3).

\begin{tabular}{|c|c|c|c|c|c|c|}
\hline \multirow{2}{*}{ Residues } & \multirow{2}{*}{ Peptide sequence } & \multirow{2}{*}{ 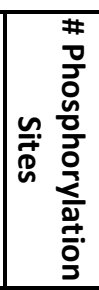 } & \multirow{2}{*}{ 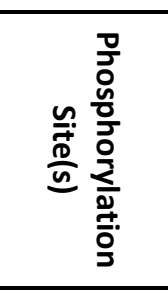 } & \multicolumn{3}{|c|}{$\begin{array}{c}\text { Relative } \\
\text { Abundance }^{\mathrm{a}}\end{array}$} \\
\hline & & & & 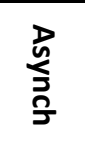 & 只 & ก \\
\hline $10-21$ & SSPDRVTQSAIK & 1 & S11 & ++ & ++ & ++++ \\
\hline $2-21$ & SGQLVQWKSSPDRVTQSAIK & 1 & S11 & ++ & ++ & ++++ \\
\hline $2-21$ & SGQLVQWKSSPDRVTQSAIK & 2 & $\mathrm{~S} 10$ \& S11 & + & + & ++ \\
\hline $2-21$ & SGQLVQWKSSPDRVTQSAIK & 2 & S11 \& T16 & - & - & + \\
\hline $22-46$ & EALHSPLADGDMNEMNVPVDPLENK & 1 & S26 & +++ & ++ & +++ \\
\hline $47-58$ & VNSTNIIEGSPK & 1 & S56 & ++++ & ++++ & ++++ \\
\hline $47-65$ & VNSTNIIEGSPKANPNPVK & 1 & S56 & ++++ & ++++ & ++++ \\
\hline $76-85$ & SLGLLDESPR & 1 & S83 & ++ & ++ & ++ \\
\hline $76-109$ & SLGLLDESPRHDDELNIEVGDNDRPNANILHNERT & 1 & S83 & ++ & ++ & ++ \\
\hline 76-109 & DRPNANILHNERTP & 1 & T110 & ++ & ND & ND \\
\hline $76-109$ & DRPNANILHNERTPDL & 1 & T110 & ++ & ND & ND \\
\hline $122-134$ & SNRTPGKENLLTK & 1 & T125 & ++++ & ++++ & ++++ \\
\hline $135-149$ & YQSSDLEDTPLMLRK & 1 & T137 & $t^{\mathrm{b}}$ & $t^{\mathrm{b}}$ & ++ \\
\hline $135-149$ & YQSSDLEDTPLMLRK & 1 & T143 & $t^{\mathrm{b}}$ & $t^{\mathrm{b}}$ & + \\
\hline $135-149$ & YQ(SSDLEDT)PLMLRK & 2 & $d$ & $t^{b}$ & - & $t^{\mathrm{b}}$ \\
\hline $150-163$ & KMTFQTPTDPLEQK & 1 & T152 & + & $t^{\mathrm{b}}$ & $t^{\mathrm{b}}$ \\
\hline $150-163$ & KMTFQTPTDPLEQK & 1 & T155 & + & $t^{b}$ & ++ \\
\hline $205-220$ & SADNYDCALEGIVTPK & 1 & S205 & $t^{\mathrm{b}}$ & + & + \\
\hline $205-220$ & SADNYDCALEGIVTPK & 1 & T218 & ++ & ++ & ++ \\
\hline
\end{tabular}


Chapter 3

Phosphorylation Analysis of Rad9

\begin{tabular}{|c|c|c|c|c|c|c|}
\hline $205-220$ & SADNYDCALEGIVTPK & 2 & $\begin{array}{c}\text { T218 \& } \\
\text { S205 }\end{array}$ & $+^{\mathrm{b}}$ & $t^{\mathrm{b}}$ & + \\
\hline $240-257$ & TQIMISAESPNSISSYDK & 1 & S248 & +++ & +++ & ++++ \\
\hline $240-257$ & TQIMISAESPNSISSYDK & 2 & $\begin{array}{c}\text { S248 \& } \\
\text { S251 }\end{array}$ & $++^{\mathrm{b}}$ & + & $t^{\mathrm{b}}$ \\
\hline $240-257$ & (TQIMISAESPNSISSY)DK & 3 & d & + & $t^{\mathrm{b}}$ & $t^{\mathrm{b}}$ \\
\hline 294-305 & SENYSSDDLRER & 1 & S298 & ++ & ++ & ++ \\
\hline 294-305 & SENYSSDDLRER & 1 & S299 & ++ & + & + \\
\hline 294-305 & SENYSSDDLRER & 2 & $\begin{array}{c}\text { S298 \& } \\
299\end{array}$ & ++ & + & + \\
\hline 306-323 & NNQIIQSNESEEINELEK & 1 & S312 & ++ & - & ++ \\
\hline $306-323$ & NNQIIQSNESEEINELEK & 1 & S315 & + & - & + \\
\hline $306-323$ & NNQIIQSNESEEINELEK & 2 & $\begin{array}{c}\text { S312 \& } \\
\text { S315 }\end{array}$ & + & - & ++ \\
\hline 331-351 & ENDVNNLDIDIN(SAVSGTPS)R & 1 & $d$ & $t^{b}$ & - & $t^{b}$ \\
\hline $352-371$ & NNAEEEM(YSSESVNNREPS)K & 1 & d & $t^{\mathrm{b}}$ & $t^{\mathrm{b}}$ & $+^{\mathrm{b}}$ \\
\hline 352-371 & NNAEEEMYSSESVNNREPSK & 2 & $\begin{array}{c}\text { S360 \& } \\
\text { S361 }\end{array}$ & $t^{\mathrm{b}}$ & $+^{\mathrm{b}}$ & + \\
\hline 382-415 & (TENNSNRSTQIVNNPRTQEMPLDSISIDTQPLS)K & 1 & d & $t^{\mathrm{b}}$ & - & $+^{\mathrm{b}}$ \\
\hline $443-456$ & GPVFH(STGQT)EEIK & 1 & d & $t^{b}$ & $t^{\mathrm{b}}$ & $t^{b}$ \\
\hline $457-480$ & TQIINSPEQNALNATFETPVTLSR & 1 & S462 & ++ & ++ & ++ \\
\hline $457-480$ & TQIINSPEQNALNATFETPVTLSR & 1 & $\begin{array}{c}\text { T471 \& } \\
474\end{array}$ & $+^{\mathrm{b}}$ & $t^{\mathrm{b}}$ & + \\
\hline 457-480 & TQIINSPEQNALNATFETPVTLSR & 2 & $\begin{array}{c}\text { S462 \& } \\
\text { T474 }\end{array}$ & - & + & + \\
\hline $481-497$ & INFEPILEVPETSSPSK & 1 & S493 & ++ & + & ++ \\
\hline 481-497 & INFEPILEVPETSSPSK & 1 & S494 & ++ & +++ & ++ \\
\hline 481-497 & INFEPILEVPETSSPSK & 2 & $\begin{array}{c}\text { S493 \& } \\
\text { S494 }\end{array}$ & + & - & - \\
\hline 481-497 & INFEPILEVPE(TS)SPSK & 2 & $\begin{array}{c}\mathrm{T} 492 / \\
\mathrm{S}^{2} 93^{\mathrm{d}}, \text { \& } \\
\text { S494 }\end{array}$ & - & + & - \\
\hline 481-497 & INFEPILEVPETSSPSK & 2 & $\begin{array}{c}\text { T492, } \\
\text { S493, \& } \\
\text { S494 }\end{array}$ & - & - & ++ \\
\hline $498-511$ & NTMSKPSNSSPIPK & 1 & S506 & + & + & + \\
\hline $498-511$ & NTMSKPSNSSPIPK & 1 & S507 & +++ & +++ & ++ \\
\hline 498-511 & NTMSKPSNSSPIPK & 2 & $\begin{array}{c}\text { S506 \& } \\
\text { S507 }\end{array}$ & ++ & $t^{b}$ & + \\
\hline $512-521$ & EKDTFNIHER & 1 & T515 & - & $t^{b}$ & + \\
\hline $543-557$ & DDIIIAG(SS)DFNNEQK & 1 & $\bar{d}$ & $t^{b}$ & $t^{\mathrm{b}}$ & $t^{b}$ \\
\hline
\end{tabular}


Chapter 3

Phosphorylation Analysis of Rad9

\begin{tabular}{|c|c|c|c|c|c|c|}
\hline $558-570$ & EI(TDRIYLQLS)GK & 1 & d & - & - & $t^{\mathrm{b}}$ \\
\hline $571-582$ & QISDSGSDETER & 1 & S575 & $t^{\mathrm{e}}$ & $t^{b}$ & + \\
\hline 571-591 & QISDSGSDETERMSPNELDTK & 1 & S584 & ++ & ++ & + \\
\hline 571-591 & QISD(SGS)DETERMSPNELDTK & 2 & $\begin{array}{c}\text { S575/ } \\
\text { S577d \& } \\
\text { S584 }\end{array}$ & + & + & + \\
\hline 571-591 & QI(SDSGSDETERMSPNELDT)K & 3 & d & $t^{\mathrm{b}}$ & $t^{\mathrm{b}}$ & $t^{\mathrm{b}}$ \\
\hline $593-620$ & ESTIMSEVELTQELPEVEEQQDLQTSPK & 1 & S618 & ++ & +++ & ++++ \\
\hline $635-647$ & (SKGNS)LQLHDDNK & 1 & d & - & - & $t^{b}$ \\
\hline $637-670$ & $\begin{array}{l}\text { ECNSDKQDGTESLDVALIEHESK/ } \\
\text { GNSLQLHDDNKECNSDK }\end{array}$ & 1 & S651 & + & + & + \\
\hline $654-670$ & QDG(TESLDVALIEHES)K & 1 & d & $t^{\mathrm{b}}$ & $t^{\mathrm{b}}$ & $t^{\mathrm{b}}$ \\
\hline 679-699 & NLMQLFPSESQEIIQNRRTIK & 1 & S688 & + & + & + \\
\hline $704-718$ & DTIEIGEEEENRSTK & 1 & S716 & + & + & ++ \\
\hline $704-723$ & DTIEIGEEEENRSTKT(SPT)K & 1 & d & $t^{\text {be }}$ & $t^{e}$ & $t^{\text {be }}$ \\
\hline 704-723 & DTIEIGEEEENRSTKTSPTK & 2 & $\begin{array}{l}\mathrm{S} 716 \& \\
720\end{array}$ & + be & $+^{e}$ & $t^{\text {be }}$ \\
\hline 704-723 & D(TIEIGEEEENRSTKTSPT)K & 3 & d & $t^{\text {be }}$ & $t^{\mathrm{be}}$ & - \\
\hline $727-738$ & RNSDLDDAASIKR & 1 & S729 & ++ & ++ & +t \\
\hline $727-738$ & RNSDLDDAASIKR & 1 & S735 & - & - & + \\
\hline 738-755 & REP(SCSITIQTGETGS)GK & 1 & d & $t^{\mathrm{b}}$ & $t^{b}$ & $t^{b}$ \\
\hline $866-873$ & G(YDT)VHLK & 1 & d & - & - & $t^{\mathrm{b}}$ \\
\hline $931-940$ & SMTNVLSPKK & 1 & S931 & + & ++ & ++ \\
\hline $931-940$ & SMTNVLSPKK & 1 & S937 & ++ & ++ & ++ \\
\hline 931-940 & SMTNVLSPKK & 2 & $\begin{array}{l}\text { S931 \& } \\
\text { S937 }\end{array}$ & $t^{\mathrm{b}}$ & $t^{\mathrm{b}}$ & + \\
\hline $931-946$ & SMTNVLSPKKHTDDEK & 1 & T943 & $t^{e}$ & $t^{e}$ & $t^{e}$ \\
\hline $931-946$ & SMTNVLSPKKHTDDEK & 2 & $\begin{array}{l}\text { S937 \& } \\
\text { T943 }\end{array}$ & $+^{\mathrm{e}}$ & $+^{e}$ & $t^{\mathrm{e}}$ \\
\hline $931-946$ & (SMTNVLS)PKKHTDDEK & 2 & $\begin{array}{c}\text { S931/ } \\
\text { T933/ } \\
\text { s937d \& } \\
\text { T943 }\end{array}$ & $t^{\text {be }}$ & $t^{\text {be }}$ & $t^{e}$ \\
\hline $947-966$ & DINTHTEVYNNEIESSSEKK & 1 & S961 & + & + & $t^{b}$ \\
\hline $947-966$ & DINTHTEVYNNEIE(SSS)EKK & 1 & $\begin{array}{l}\text { S961 \& } \\
963\end{array}$ & - & + & + \\
\hline $947-966$ & DINTHTEVYNNEIESSSEKK & 2 & $\begin{array}{l}\text { S961, 962, } \\
\quad \& 963\end{array}$ & $t^{\mathrm{b}}$ & $t^{\mathrm{b}}$ & + \\
\hline 975-994 & DALAEHAGAPSLLFSSGEIR & 1 & $\begin{array}{l}\text { S989 \& } \\
\text { S990 }\end{array}$ & $t^{\mathrm{b}}$ & - & + \\
\hline
\end{tabular}




\begin{tabular}{|c|c|c|c|c|c|c|}
\hline $975-994$ & DALAEHAGAPSLLFSSGEIR & 1 & $\begin{array}{c}\text { S985, } \\
\text { S99, }\end{array}$ & & + & - \\
\hline $\begin{array}{c}1132- \\
1139\end{array}$ & LSLDSPSK & 1 & $\begin{array}{c}\text { S1136 \& } \\
\text { S1138 }\end{array}$ & $\mathrm{ND}^{\mathrm{c}}$ & + & $++^{\mathrm{b}}$ \\
\hline
\end{tabular}

Table 3.3 - Rad9 phosphorylation sites assigned by manual interpretation of collision-activated dissociation and/or electron transfer dissociation mass spectra. ${ }^{a}$ Phosphorylated peptide levels are reported as \% total peptide abundance detected, $(++++)=70-100 \%,(+++) 35-69 \%,(++)=6-34 \%,(+)=1-5 \%,(-)$ no phosphorylated peptide detected. \% Total abundances were calculated from ion currents observed in the main beam mass spectra [(phosphorylated peptide ion current)/(phosphorylated peptide + non-phosphorylated peptide ion current) $\times(100)]$. bPhosphorylated peptide containing this phosphorylation site was assigned by HPLC retention time and correct accurate mass, only. ${ }^{c}$ Nonphosphorylated and phosphorylated peptides were not detected (ND). dPTM site is located on S/T/Y within parentheses. Putative CDK sites are highlighted.

A total of 63 unique Rad9 phosphorylation sites were detected amongst the three cell cycle samples and the majority were detected in all three. The collective trend mostly showed phosphorylation levels in equal abundances throughout the cell cycle or increasing in G2. Over half of the phosphorylation sites detected were previously unknown, including 7 CDK. Of the 63 phosphorylation sites detected, 50 were unambiguously site-mapped (Table 3.4).

\begin{tabular}{|c|c|c|}
\hline 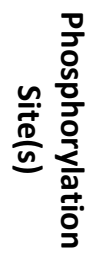 & 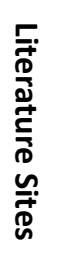 & 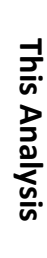 \\
\hline S10 & $x$ & $x$ \\
\hline S11 & $x$ & $x$ \\
\hline T16 & & $x$ \\
\hline S26 & $x$ & $x$ \\
\hline T50 & $x$ & \\
\hline S56 & $x$ & $x$ \\
\hline S70 & $x$ & \\
\hline S76 & $x$ & \\
\hline S83 & $x$ & $x$ \\
\hline T110 & & $x$ \\
\hline T125 & & $x$ \\
\hline S137 & $x$ & $x$ \\
\hline S138 & $x$ & $x$ \\
\hline T143 & & $x$ \\
\hline
\end{tabular}




\begin{tabular}{|c|c|c|}
\hline T152 & & $X$ \\
\hline T155 & $X$ & $X$ \\
\hline S188 & $X$ & \\
\hline S205 & $X$ & $X$ \\
\hline T218 & $X$ & $X$ \\
\hline S248 & $X$ & $X$ \\
\hline S251 & & $X$ \\
\hline S312 & $X$ & $X$ \\
\hline S315 & $X$ & $X$ \\
\hline S328 & $X$ & \\
\hline T348 & $X$ & $?$ \\
\hline S360 & & $X$ \\
\hline S361 & & $X$ \\
\hline T398 & $X$ & \\
\hline S414 & $X$ & \\
\hline S416 & $X$ & $X$ \\
\hline S435 & $X$ & \\
\hline T457 & $X$ & \\
\hline S462 & $X$ & $X$ \\
\hline T471 & $X$ & $X$ \\
\hline T474 & & $X$ \\
\hline S479 & $X$ & \\
\hline T492 & & $X$ \\
\hline S493 & & \\
\hline S494 & & $X$ \\
\hline S506 & & $X$ \\
\hline S507 & $X$ \\
\hline T515 & & $X$ \\
\hline S537 & $X$ & $X$ \\
\hline T541 & $X$ & $X$ \\
\hline S550 & $X$ & $X$ \\
\hline S568 & $X$ & $X$ \\
\hline S575 & & $X$ \\
\hline S584 & & $X$ \\
\hline S698 & $X$ & $X$ \\
\hline
\end{tabular}




\begin{tabular}{|c|c|c|}
\hline S651 & $X$ & $X$ \\
\hline S688 & $X$ & $X$ \\
\hline S716 & & $X$ \\
\hline S720 & & $X$ \\
\hline S729 & $X$ & $X$ \\
\hline S735 & & $X$ \\
\hline S741 & $X$ & $X$ \\
\hline T807 & $X$ & \\
\hline S931 & $X$ & $X$ \\
\hline S937 & $X$ & $X$ \\
\hline T943 & & $X$ \\
\hline S961 & & $X$ \\
\hline S962 & & $X$ \\
\hline S963 & & $X$ \\
\hline S985 & & $X$ \\
\hline S989 & $X$ & $X$ \\
\hline S990 & $X$ & $X$ \\
\hline S1136 & $X$ & $X$ \\
\hline S1138 & & X \\
\hline & Total & Total \\
\cline { 2 - 3 } & 45 & 50 \\
\hline & & Known \\
\hline & & Novel \\
\hline
\end{tabular}

Table 3.4-Previously known phosphorylation sites 28,29 and those detected in this analysis are indicated by $X$. This analysis revealed 27 previously unknown phosphorylation sites on Rad9, including 7 CDK sites. All CDK sites are highlighted.

All 9 CAD CDK sites were observed to be phosphorylated. Phosphorylation of S11 appeared to act constitutively with S10 or T16 phosphorylation. CDK-dependent phosphorylation of S11 is critical for the interaction between Rad9 and Dpb11, a scaffold/mediator protein required for Rad9's checkpoint activity in mitosis ${ }^{30}$. CDK phosphorylation of Rad9 S11 may enhance the interaction of Rad9 with kinases responsible for phosphorylating S10 and/or T16. This indicates that Dpb11-dependant Rad9 checkpoint activity requires more kinases than CDK alone. Phosphorylation on CDK sites T110, T125, and T143 was previously unreported, but detected on each in this study. There was a sharp contrast in the 
abundance of phosphorylation between T125 and T143 as the levels were nearly stoichiometric in all samples for the former and $<5 \%$ in all samples for the latter. Phosphorylation was also localized on S137 in the absence of T143. A di-phosphorylated form of peptide YQSSDLEDTPLMLRK, containing both modifications, was detected in similar abundance. It is likely that both modifications are present together, but the sites could not be unambiguously localized in the di-phosphorylated form. Another formerly unreported phosphorylation site, T152, was detected on the same peptide containing CDK site T155, though phosphorylation of one was only detected in the absence of the other. Modification of these sites may be mutual exclusive of one another.

Novel phosphorylation was detected within residues $448-452$ in the SO/TQ cluster domain (SCD) of Rad9. SCD domains are recognized as a core characteristic of DNA-damageresponse proteins and serve as templates for DNA-damage-induced phosphorylation by ATM/ATR kinases ${ }^{31}$. This site does not fit the SQ/TQ motif, however. Another SCD phosphorylation site was detected amongst residues 382-414, which contains the TQ-consensus site T398, previously reported to be phosphorylated.

Two novel phosphorylation sites were detected on Rad9's Tudor domain. This domain is essential for Rad9's ability to interacting with chromatin, and both single- and double-stranded DNA during checkpoint activation and DNA repair ${ }^{32,33}$. Phosphorylation sites T942 and Y867/T69 were previously unknown.

The C-terminus of Rad9 contains a BRCA1 carboxyl terminus (BRCT) domain which is essential for its function at least in part by facilitating Rad9 oligomerization ${ }^{34}$. Novel phosphorylation was detected on S1138 and on the known site, S1136, in the absence of the former. 
Rad9 is heavily phosphorylated throughout the length of its sequence and on all four defined functional domains (Figure 3.3). Novel sites were also detected throughout Rad9 and on all domains including the most heavily modified, CAD, critical for Chk1 activation.

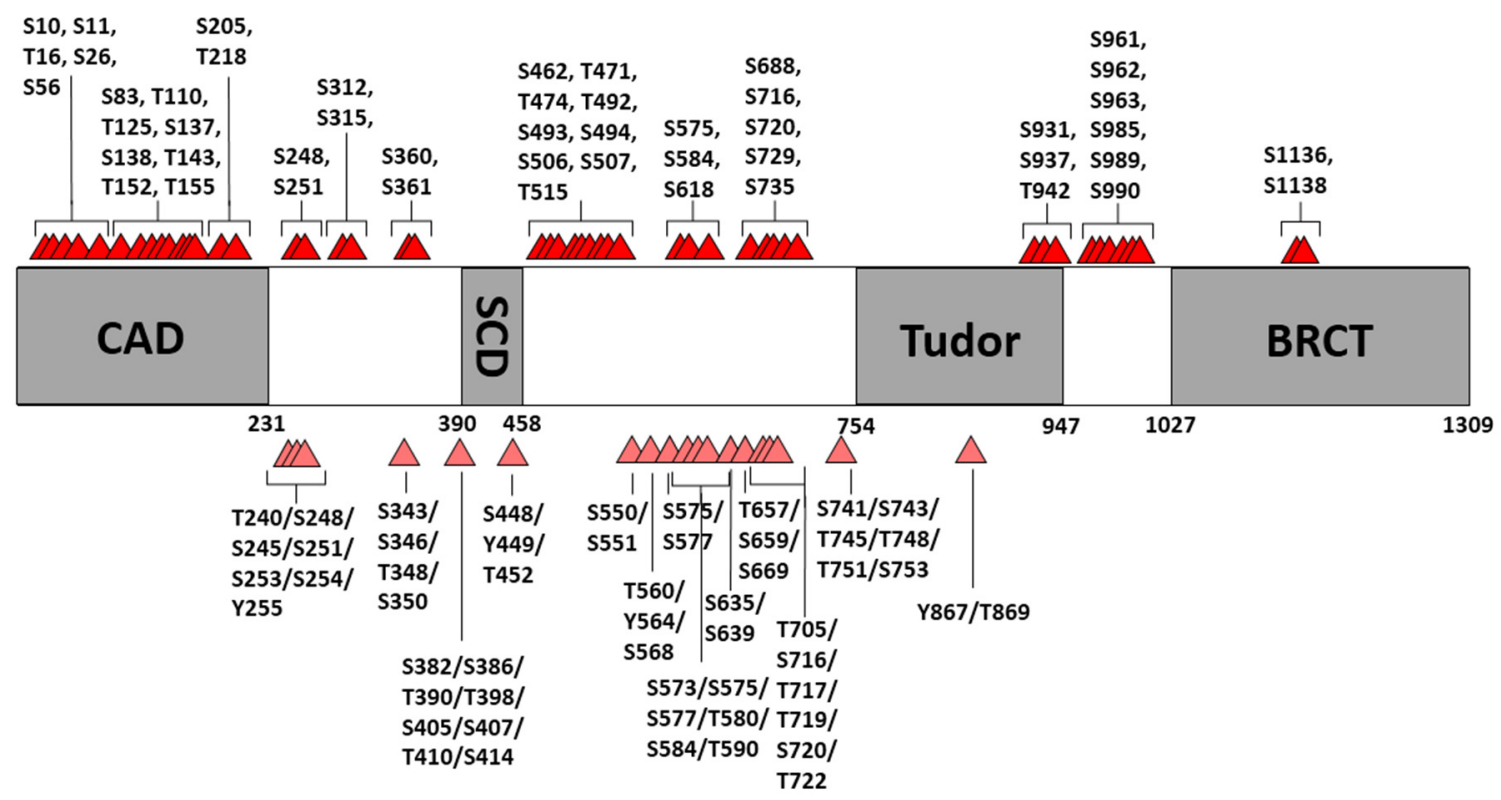

Figure 3.3 - Schematic of all Rad9 phosphorylation sites detected in this study. Dark red triangles (top) indicate all unambiguously localized sites, and the light red triangles (bottom) indicate the regional localization of all additional phosphorylation sites detected.

\subsubsection{Biological role of CAD phosphorylation in Chk1 activation}

Molecular biology experiments were conducted by Lowndes and coworkers to investigate the function of CDK and its phosphorylation sites on Rad9 CAD. CDK sites 1-9 located in the CAD (Figure 3.4A) were confirmed to be phosphorylated by cdc28 by in vitro phosphorylation by four different cdc28 cyclin complexes (Figure 3.4B) ${ }^{24}$. Complex cdc28/clb2 exhibited the highest activity.

Next, the effect of DNA damage-induced phosphorylation of CAD CDK sites on Chk1 activation was examined in vivo. Tagged Chk1-3HA was detected by western blot from 
asynchronous, G1-, and G2/M-arrested cells treated with bleocin. Rad9 CDK sites 1-9 were

mutated to alanine and Chk1 activation was compared to cells containing wild-type Rad9. While some residual Chk1 activity remained in G1-arrested cells, Chk1 activity was eliminated in G2/M cells following DNA-damage as observed by its decrease in phosphorylation (Figure 3.4C-E) ${ }^{24}$.

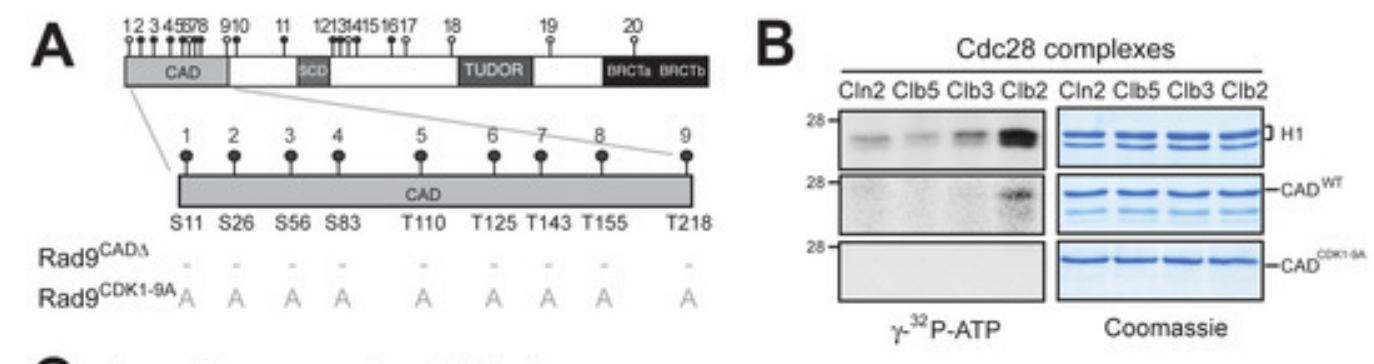

C Asynchronous cells + DNA damage

wild type $\quad \operatorname{rad9} C D K 1-9 A \quad \operatorname{rad9CAD\Delta } \operatorname{rad} 9 \Delta$

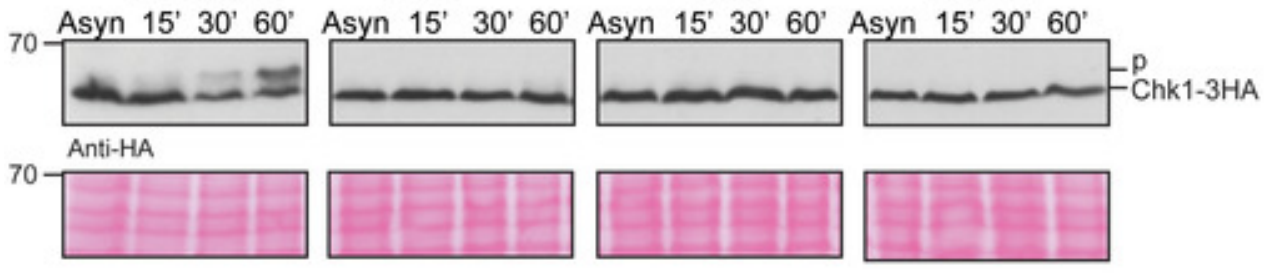

D G1 cells + DNA damage

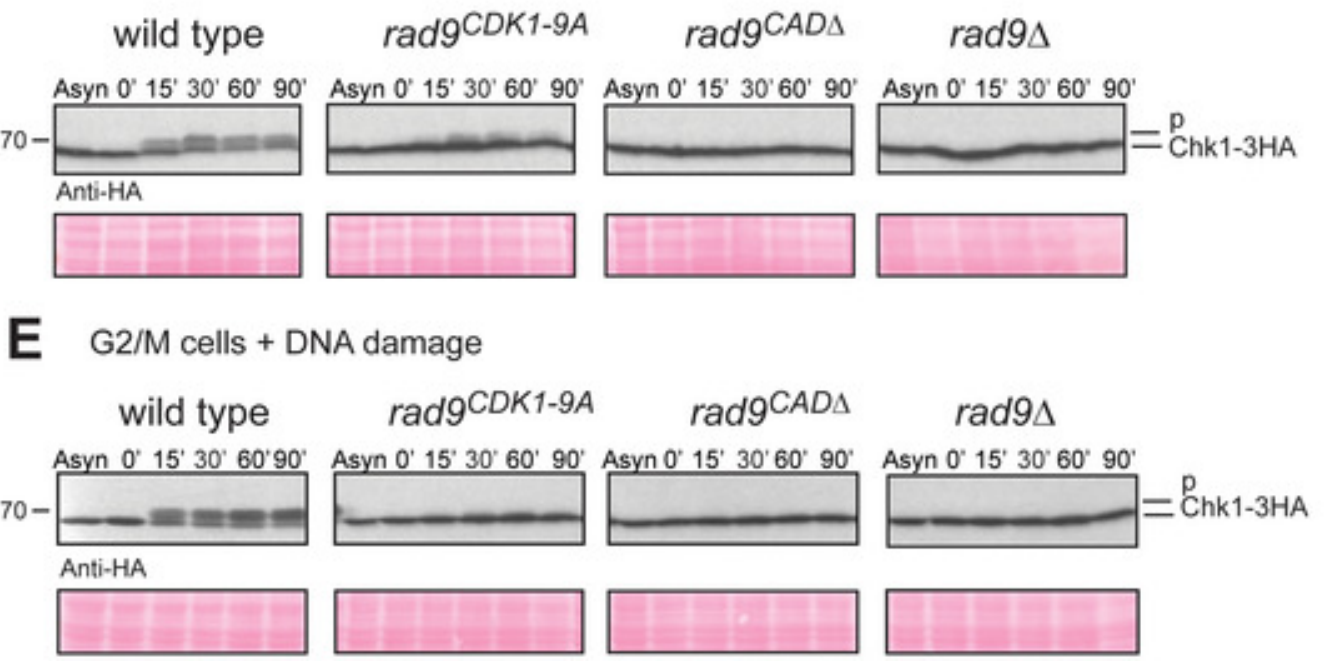

Figure 3.4 - (A) Schematic of Rad9 CAD highlighting Cdc28 consensus sites 1-9 mutated to Ala in Rad9CDK1-9A protein. (B) Cdc28/Clb2 produces the highest level of in vitro phosphorylation of Rad9 CADWT. (C) DNA-damage-dependent phosphorylation of Chk1 is reduced or eliminated in Rad9CDK1-9A, Rad9CADL, Rad9D cells. (D\&E) - Chk1 activation is principally dependent on CDK consensus sites 1-9 in G2/M cells. Asynchronously-growing cells were treated with bleocin for the indicated times and Chk1 phosphorylation was indicated by anti-HA western blot. G1 and G2/M cells were arrested by $\alpha$-factor and nocodazole, respectively. Figure reproduced from ${ }^{24}$. 
Furthermore, CDK-dependent activation of Chk1 was found to control Rad9/Chk1 interaction. It was previously known that Chk1 activation required Cdc28 activity in response to double-strand breaks ${ }^{35,36}$. In this study, Cdc28-as1 cells which can be rapidly inhibited, arrested, and their DNA damaged, were used to investigate whether Cdc28 can activate Chk1 independently of CDC28-dependent regulation of double-strand breaks ${ }^{24}$. Cells were arrested in G2/M, DNAdamaged, and several components of the DDR were examined by western blot for phosphorylation in the presence and absence of Cdc28 activity. 4-NQO was used to induce single-strand breaks and DNA adducts while bleocin induced double-strand breaks. Cdc28 activity was required for Chk1 activation in response to either agent. Rad9 and Rad53 were activated independently of Cdc28 activity in response to 4-NQO, and consistent with previous studies, Rad53 activation was dependent on Cdc28 in response to bleocin-induced doublestrand breaks (Figure 3.5) ${ }^{36}$. These results provided further evidence that Cdc28-dependent phosphorylation of Rad9 is required for Chk1 activation. 


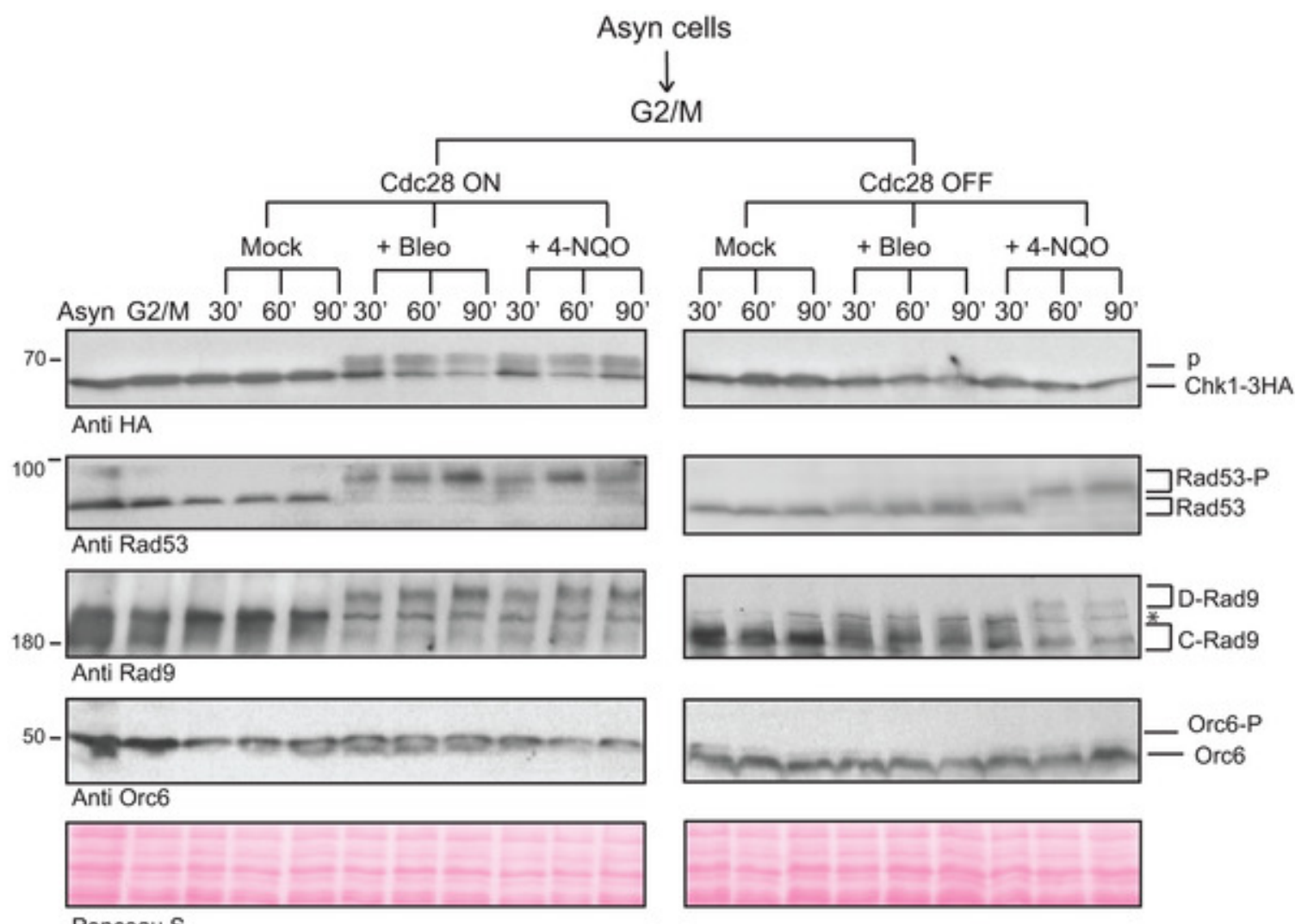

Ponceau S

Figure 3.5 - Chk1 activity was dependent on Cdc28 upon DNA damage separately induced by bleocin or 4-NQO. Otc6 phosphorylation marked Cdc28 inactivation. Figure reproduced from ${ }^{24}$.

The mechanism of Rad9-dependent activation of Chk1 was further investigated by yeast two-hybrid assays ${ }^{24}$. The necessity of CDK sites 1-9 for Rad9/Chk1 interaction was tested in G1and G2/M-arrested cells (Figure 3.6A), and they were required in both cell stages. This interaction was also Cdc28-dependent in G2/M yet was Cdc28-independent in G1 (Figure 3.6B). A reciprocal co-immunoprecipitation experiment validated these results by showing that a portion of Rad9 co-immunoprecipitated with Chk1 and vice versa, and this was abolished by $\operatorname{Rad9}^{\mathrm{CDK1}-9 \mathrm{~A}}$ (data not shown). 

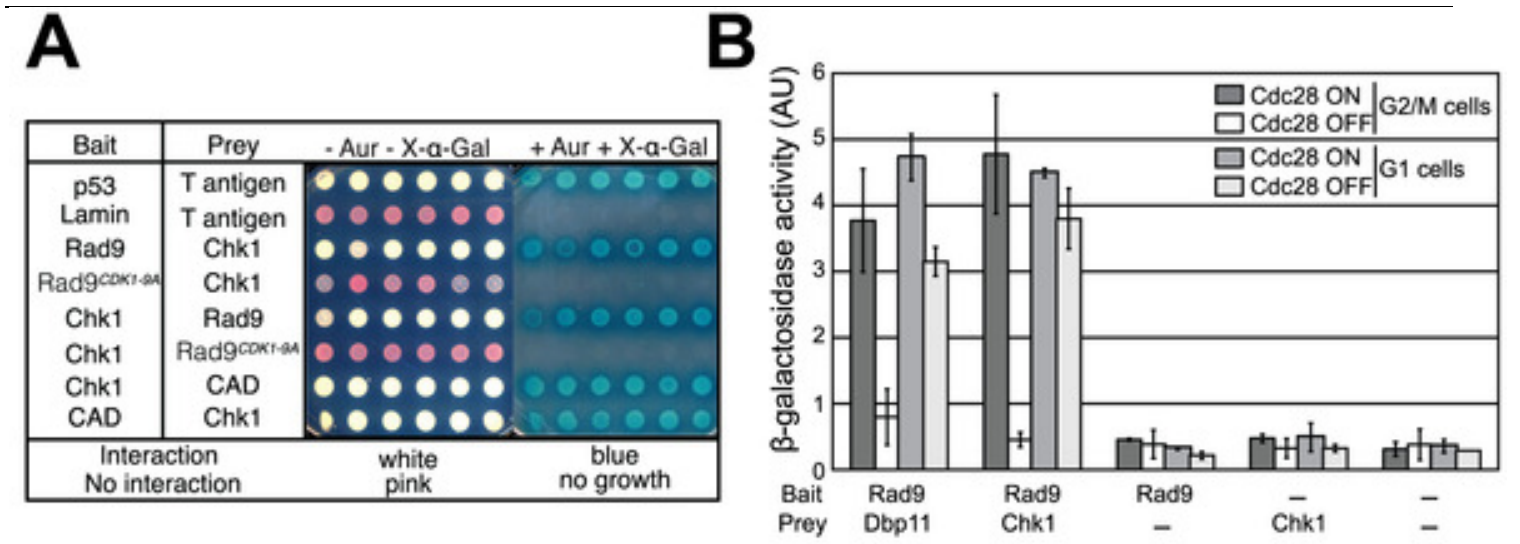

Figure 3.6 - (A) Rad9/Chk1 interaction was dependent on CDK1-9 based on yeast two-hybrid assay in vivo. (B) Rad9/Chk1 interaction was dependent on Cdc28 in G2/M and was Cdc28-independent in G1. Figure reproduced from ${ }^{24}$.

Yeast two-hybrid and peptide pull-down experiments were lastly used to determine which specific CAD CDK sites facilitated Rad9/Chk1 interaction. CAD 1-231 mutant constructs were generated in which CDK1-9 sites were mutated to Ala and individually mutated back to the wild type residue. The ability of each 'reversion' CAD mutant to interact with Chk1 was assessed by yeast two-hybrid assay (Figure 3.7A). The most prominent rescues of CAD/Chk1 interaction were made by reversions of T125A and T143A to wild-type. Phosphorylated and nonphosphorylated 35-amino acid peptides containing T125 and T143 were constructed to test their ability to pull-down Chk1. The pT143 peptides pulled Chk1 down most efficiently while peptides containing pT125 demonstrated much reduced interaction independently of T143 phosphorylation (Figure 3.7B). This suggests that phosphorylated of T143 is a stimulator of Rad9/Chk1 interaction and phosphorylation of T125 is an inhibitor ${ }^{24}$. Mass spectrometric analysis of Rad9 indicated that T125 was heavily phosphorylated in vivo and only a small fraction of Rad9 protein was phosphorylated on T143. This suggests that phosphorylation of a small population of Rad9 is responsible for the DNA-damage-independent interaction of Rad9 and Chk1, and is consistent with the co-immunoprecipitation experiments in which only a minority 
of Rad9 bound to Chk1 and vice versa.

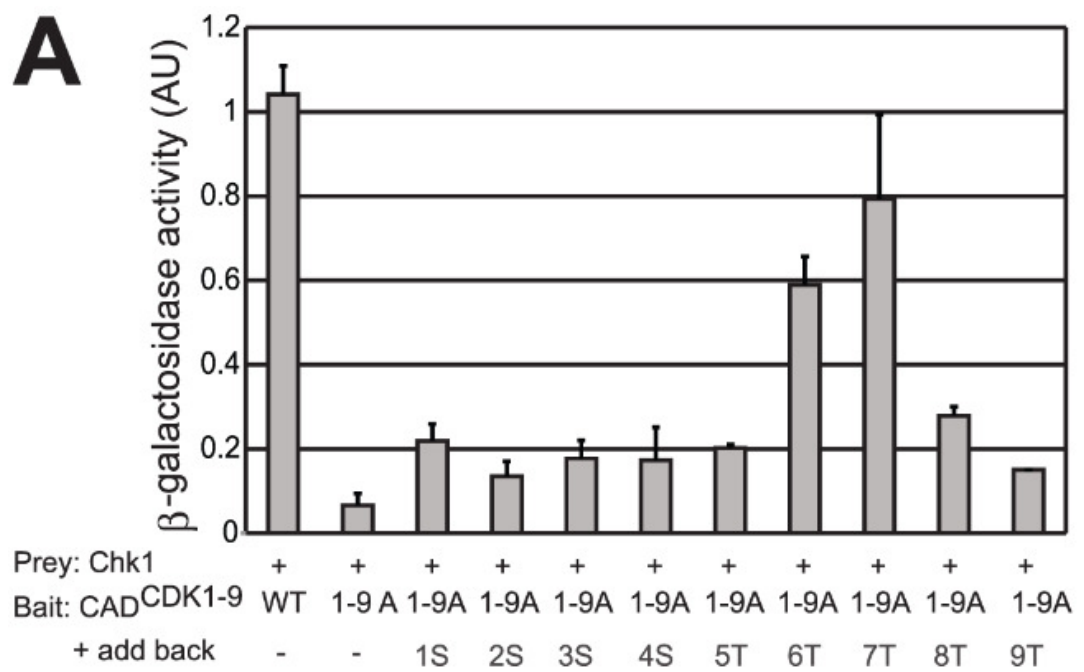

B

125

143

Un-P A-N-F-F-K-S-N-R- T -P-G-K-E-N-L-L-T-K-Y-Q-S-S-D-L-E-D- T -P-L-M-L-R-K-K-M

T125p A-N-F-F-K-S-N-R-TP-P-G-K-E-N-L-L-T-K-Y-Q-S-S-D-L-E-D- T -P-L-M-L-R-K-K-M

T143p A-N-F-F-K-S-N-R- T-P-G-K-E-N-L-L-T-K-Y-Q-S-S-D-L-E-D-Tp-P-L-M-L-R-K-K-M

T125p T143p A-N-F-F-K-S-N-R-Tp-P-G-K-E-N-L-L-T-K-Y-Q-S-S-D-L-E-D-Tp-P-L-M-L-R-K-K-M

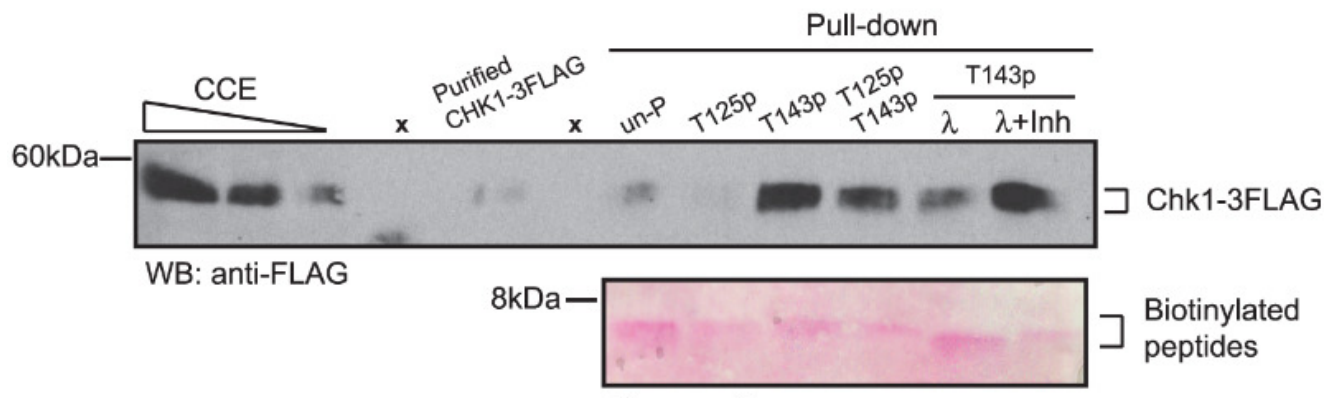

Ponceau S

Figure 3.7 - (A) Yeast-two hybrid assay demonstrated that T125 and T143 most contributed to CAD/Chk1 interaction of CDK1-9 sites. (B) - Peptides containing pT143 most effectively pulled down Chk1 while pT125 showed an inhibitory effect. Figure reproduced from ${ }^{24}$. 


\subsection{Conclusion}

Mass spectrometric analysis of DNA damage mediator Rad9 revealed that 63 residues were in vivo phosphorylated independent of DNA damage (Table 3.5) and 34-40 of these were previously unreported.

\begin{tabular}{|c|c|c|c|c|}
\hline \multirow[b]{2}{*}{ 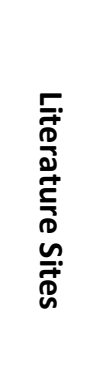 } & \multicolumn{4}{|c|}{ This study } \\
\hline & \multicolumn{2}{|c|}{ 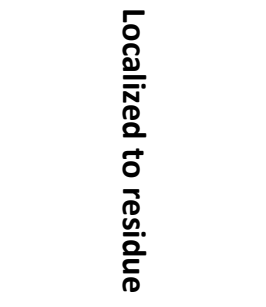 } & \multicolumn{2}{|c|}{ 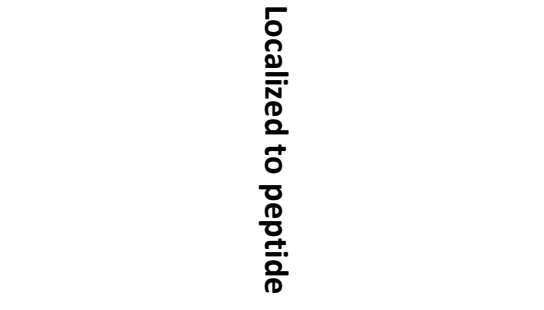 } \\
\hline Total & \multicolumn{2}{|c|}{ Total } & \multicolumn{2}{|c|}{ Total } \\
\hline 45 & \multicolumn{2}{|c|}{50} & \multicolumn{2}{|c|}{13} \\
\hline & Known & Novel & Novel & Undetermined \\
\hline & 23 & 27 & 7 & 6 \\
\hline & \multicolumn{4}{|c|}{ Summary } \\
\hline & Known & Novel & Undetermined & Total \\
\hline & 23 & 34 & 6 & 63 \\
\hline
\end{tabular}

Table 3.5 - 50 of 63 phosphorylation sites detected were localized to the Ser/Thr residue modified and 27 of these were previously unreported. In total, 34-40 unreported phosphorylation sites were detected.

This represents the largest number of phosphorylation sites reported on Rad9 in any single study. Phosphorylation was detected on CDK sites 1-9 in the Rad9 Chk1 activation domain and phosphorylation was ambiguously localized to all but one of the 10 CDK sites located outside this domain as well. Phosphorylation of CDK sites T125 and T143 was found to be especially critical for Chk1 activation by regulating the interaction between Rad9 and Chk1. This study has further elucidated the role of Rad9 and the mechanism by which its Chk1 activating domain regulates Rad/Chk1 interaction through Cdc28/Clb-dependent phosphorylation. Abreu et al. 


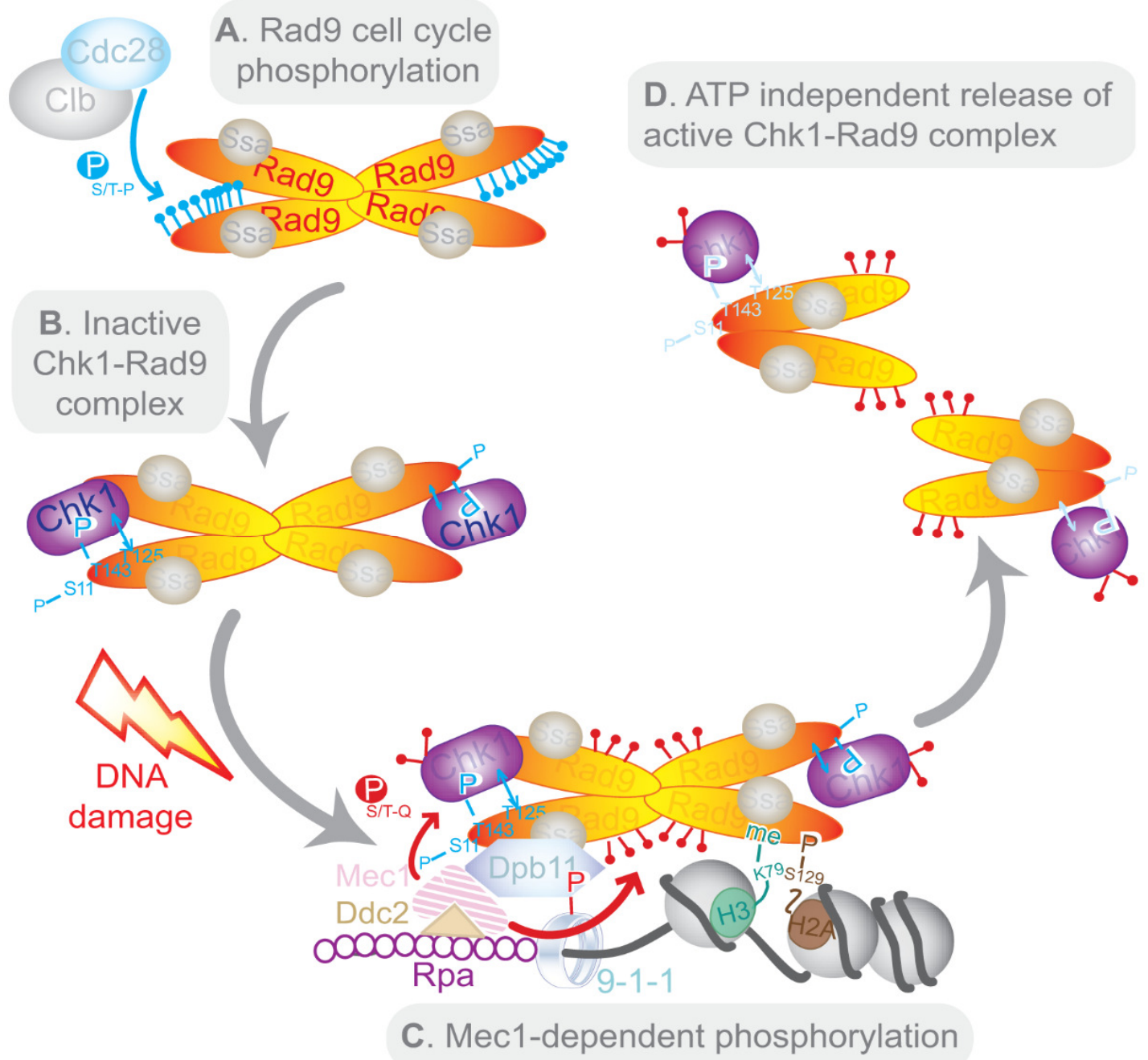

Figure 3.8 - Model of Chk1 activation in response to DNA damage. Rad9 CAD phosphorylation by Cdc28/Clb (A) regulates Rad9/Chk1 interaction independent of DNA-damage and is most promoted by pT143 and no phosphorylation on T125 (B). Rad9 complexes are recruited to sites of DNA damage and recognize specific histone marks. Other Rad9 phosphorylation sites stimulate its interaction with Dpb11, recruited in a 9-1-1-complex-dependent manner (C). These together enhance Mec1 activity and remodeling of Rad9 and Chk1, resulting in the in cis autophosphorylation of Chk1 and release of the hyper-phosphorylated Rad9/Chk1 complex. In contrast to Rad9/Rad53 complexes, Rad9-Chk1 release was found to then proceed in an ATP-independent manner (D). Figure reproduced from ${ }^{24}$. 


\subsection{References}

1. Harper, J. W. \& Elledge, S. J. The DNA damage response: ten years after. Mol. Cell 28, 739-745 (2007).

2. Halazonetis, T. D., Gorgoulis, V. G. \& Bartek, J. An oncogene-induced DNA damage model for cancer development. Science 319, 1352-1355 (2008).

3. Durocher, D. \& Jackson, S. P. DNA-PK, ATM and ATR as sensors of DNA damage: variations on a theme? Curr. Opin. Cell Biol. 13, 225-231 (2001).

4. Stracker, T. H., Usui, T. \& Petrini, J. H. J. Taking the time to make important decisions: The checkpoint effector kinases Chk1 and Chk2 and the DNA damage response. DNA Repair 8, 1047-1054 (2009).

5. Saka, Y., Esashi, F., Matsusaka, T., Mochida, S. \& Yanagida, M. Damage and replication checkpoint control in fission yeast is ensured by interactions of Crb2, a protein with BRCT motif, with Cut5 and Chk1. Genes Dev. 11, 3387-3400 (1997).

6. FitzGerald, J. E., Grenon, M. \& Lowndes, N. F. 53BP1: function and mechanisms of focal recruitment. Biochem. Soc. Trans. 37, 897-904 (2009).

7. Jungmichel, S. \& Stucki, M. MDC1: The art of keeping things in focus. Chromosoma 119, 337-349 (2010).

8. Weinert, T. A. \& Hartwell, L. H. The RAD9 gene controls the cell cycle response to DNA damage in Saccharomyces cerevisiae. Science 241, 317-322 (1988).

9. O'Shaughnessy, A. M. et al. in Methods in Enzymology 131-150 (Academic Press.

10. Finn, K., Lowndes, N. F. \& Grenon, M. Eukaryotic DNA damage checkpoint activation in response to double-strand breaks. Cell Mol. Life Sci. 69, 1447-1473 (2012).

11. Usui, T., Foster, S. S. \& Petrini, J. H. Maintenance of the DNA-damage checkpoint requires DNA-damage-induced mediator protein oligomerization. Mol. Cell 33, 147-159 (2009).

12. Gilbert, C. S., Green, C. M. \& Lowndes, N. F. Budding yeast Rad9 is an ATP-dependent Rad53 activating machine. Mol. Cell 8, 129-136 (2001).

13. Pellicioli, A. \& Foiani, M. Signal transduction: how rad53 kinase is activated. Curr. Biol. 15, R769-71 (2005).

14. Tapia-Alveal, C., Calonge, T. M. \& O'Connell, M. J. Regulation of chk1. Cell. Div. 4, 8-1028-4-8 (2009).

15. Blankley, R. T. \& Lydall, D. A domain of Rad9 specifically required for activation of Chk1 in budding yeast. J. Cell. Sci. 117, 601-608 (2004).

16. Rappold, I., Iwabuchi, K., Date, T. \& Chen, J. Tumor suppressor p53 binding protein 1 (53BP1) is involved in DNA damage-signaling pathways. J. Cell Biol. 153, 613-620 (2001).

17. Vialard, J. E., Gilbert, C. S., Green, C. M. \& Lowndes, N. F. The budding yeast Rad9 checkpoint protein is subjected to Mec1/Tel1-dependent hyperphosphorylation and interacts with Rad53 after DNA damage. EMBO J. 17, 5679-5688 (1998).

18. Ubersax, J. A. et al. Targets of the cyclin-dependent kinase Cdk1. Nature 425, 859-864 (2003).

19. Morgan, D. O. Cyclin-dependent kinases: engines, clocks, and microprocessors. Annu. Rev. Cell Dev. Biol. 13, 261291 (1997).

20. Nasmyth, K. At the heart of the budding yeast cell cycle. Trends in Genetics 12, 405-412 (1996).

21. Albuquerque, C. P. et al. A multidimensional chromatography technology for in-depth phosphoproteome analysis. Mol. Cell. Proteomics 7, 1389-1396 (2008).

22. Holt, L. J. et al. Global analysis of Cdk1 substrate phosphorylation sites provides insights into evolution. Science 325, 1682-1686 (2009). 
23. Smolka, M. B. et al. Dynamic changes in protein-protein interaction and protein phosphorylation probed with amine-reactive isotope tag. Mol. Cell. Proteomics 4, 1358-1369 (2005).

24. Abreu, C. M. et al. Site-specific phosphorylation of the DNA damage response mediator rad9 by cyclin-dependent kinases regulates activation of checkpoint kinase 1. PLoS Genet. 9, e1003310 (2013).

25. Hall, E. H. et al. Comprehensive analysis of phosphorylation sites in Tensin1 reveals regulation by p38MAPK. Mol. Cell. Proteomics 9, 2853-2863 (2010).

26. Udeshi, N. D., Compton, P. D., Shabanowitz, J., Hunt, D. F. \& Rose, K. L. Methods for analyzing peptides and proteins on a chromatographic timescale by electron-transfer dissociation mass spectrometry. Nat. Protoc. 3, 17091717 (2008).

27. Syka, J. E., Coon, J. J., Schroeder, M. J., Shabanowitz, J. \& Hunt, D. F. Peptide and protein sequence analysis by electron transfer dissociation mass spectrometry. Proc. Natl. Acad. Sci. U. S. A. 101, 9528-9533 (2004).

28. Smolka, M. B., Albuquerque, C. P., Chen, S. H. \& Zhou, H. Proteome-wide identification of in vivo targets of DNA damage checkpoint kinases. Proc. Natl. Acad. Sci. U. S. A. 104, 10364-10369 (2007).

29. Albuquerque, C. P. et al. A multidimensional chromatography technology for in-depth phosphoproteome analysis. Mol. Cell. Proteomics 7, 1389-1396 (2008).

30. Granata, M. et al. Dynamics of Rad9 chromatin binding and checkpoint function are mediated by its dimerization and are cell cycle-regulated by CDK1 activity. PLoS Genet. 6, 10.1371/journal.pgen.1001047 (2010).

31. Traven, A. \& Heierhorst, J. SQ/TQ cluster domains: concentrated ATM/ATR kinase phosphorylation site regions in DNA-damage-response proteins. Bioessays 27, 397-407 (2005).

32. Grenon, M. et al. Docking onto chromatin via the Saccharomyces cerevisiae Rad9 Tudor domain. Yeast 24, 105119 (2007).

33. Lancelot, N. et al. The checkpoint Saccharomyces cerevisiae Rad9 protein contains a tandem tudor domain that recognizes DNA. Nucleic Acids Res. 35, 5898-5912 (2007).

34. Soulier, J. \& Lowndes, N. F. The BRCT domain of the S. cerevisiae checkpoint protein Rad9 mediates a Rad9-Rad9 interaction after DNA damage. Current Biology 9, 551-S2 (1999).

35. Enserink, J. M. \& Kolodner, R. D. An overview of Cdk1-controlled targets and processes. Cell. Div. 5, 11-1028-5-11 (2010).

36. Ira, G. et al. DNA end resection, homologous recombination and DNA damage checkpoint activation require CDK1. Nature 431, 1011-1017 (2004). 